\title{
Two-Phase Flow Through Small Branches in a Horizontal Pipe with Stratified Flow
}

\author{
C. Smoglie \\ Institut für Reaktorbauelemente
}

Kernforschungszentrum Karlsruhe 


\section{KERNFORSCHUNGSZENTRUM KARLSRUHE}

Institut fur Reaktorbauelemente

KffK 3861

Two-Phase Flow Through Small Branches in a Horizontal Pipe with Stratified Flow

Cecilia Smoglie

This report is a translation of a dissertation presented to the Faculty of Mechanical Engineering at the University of Karlsruhe December 1984

Kernforschungszentrum Karlsruhe GmbH, Karlsruhe 
Als Manuskript vervielfältigt

Für diesen Bericht behalten wir uns alle Rechte vor

Kernforschungszentrum Karlsruhe $\mathrm{GmbH}$

ISSN 0303-4003 
SUMMARY

In the field of nuclear reactor safety the occurrence of a small break in a horizontal coolant pipe is of great importance. This report presents the description and results of experiments designed to determine the mass flow rate and quality through a small break at the bottom, the top or the side of a main pipe with stratified gas-liquid flow. The break was simulated by circular branches with diameters of $6,8,12$ or $20 \mathrm{~mm}$, perpendicular to the main pipe with a diameter of $206 \mathrm{~mm}$. The experiments were performed with air-water flows at ambient temperature and a maximal pressure of $0.5 \mathrm{MPa}$.

If the interface level is far below (above) the branch, only single-phase gas (liquid) flow enters the branch. For smaller distances the interface is 1ocally deformed because of the pressure decrease due to the fluid acceleration near the branch inlet (Bernoul1i effect) and liquid (gas) can be entrained.

This report contains photographs illustrating the flow phenomena as well as a general correlation to determine the beginning of entrainment. Results are presented on the branch mass flow rate and quality as a function of a normalized distance between the interface and the branch inlet.

A mode1 was developed which enables to predict the branch quality and mass flux. Results from air-water flow through horizontal branches, were extrapolated for steam water flow at high pressure with critical branch mass flux. 
Zweiphasenströmungen durch kleine Abzweige in einem horizontalen Rohr mit geschichteter Gas-Flüssigkeits-Strömung

\section{Zusammenfassung}

Im Bereich der Reaktorsicherheit ist der Kühlmittelverlustunfall, verursacht durch kleine Lecks in der horizontalen Hauptkühlmittelleitung, von großer Bedeutung. Dieser Bericht enthält die Beschreibung und die Ergebnisse von Experimenten zur Bestimmung des Massenstroms und des Dampfgehalts durch ein kleines Leck, das sich oben, unten oder an der Seite einer waggerechten llauptkühlmittelleitung befindet, in der eine geschichtete Gas-Fliissigkeitsströmung vorhanden ist. Das Leck wurde durch kreisförmige Rohrabzweige mit Durchmessern von $6,8,12$ oder $20 \mathrm{~mm}$ simuliert, die senkrecht zur Achse des Hauptrohres angebracht wurden. Die Experimente wurden mit Luft-Wasser Strömung bei Umgebungstemperatur und einem maximalen Druck von 0,5 MPa durchgeführt.

Wenn sich der Wasserspiegel weit unterhalb (oberhalb) des Rohrabzweigs befindet, gelangt nur Gas (Fliissigkeit) in den Abzweig. Bei kleineren Abständen zwischen Abzweig und Wasserspiegel wird die Wasseroberfläche lokal verformt und Flüssigkeit (Gas) kann mitgerissen werden. Die Verformung wird durch den Druckabfall bewirkt, der durch die starke Beschleunigung des Fluids in der Nähe des Abzweigs verursacht wird (Bernoulli Effekt).

Dieser Bericht enthält Fotos, die die Strömungsphänomene veranschaulichen, sowie eine allgemein giiltige Korrelation, um den Beginn des Mitrisses zu bestimmen. Außerdem werden experimentelle Ergebnisse über Massenstromdichte und Dampfgehalt im Abzweig als Funktion eines normalisierten Abstandes gezeigt und die Abhängigkeit dieser Größen in empirischen Beziehungen beschrieben. Die Ergebnisse für horizontale Abzweige wurden übertragen auf DampfWasser-Strömungen bei hohen Drücken und kritischem Massenstrom im Abzweig. 


\section{Contents}

Nomenclature 3

Page

1

Introduction

2

Experimental Setup

3

Description of the Experiments

$\begin{array}{lll}3.1 & \text { General Characteristics } & 17\end{array}$

3.1.1 Experimental Me.thod 17

3.1.2 Measurements Accuracy 18

3.2 Experiments with Downward Branches 19

3.3 Experiments with Upward Branches 20

3.4 Experiments with Horizontal Branches 21

4 Flow Phenomena 22

4.1 Downward Branch 22

4.2 Upward Branch 24

4.3 Horizontal Branch 32

4.4 Transition from Stratified to Slug Flow 34

5 Beginning of Gas and Liquid Entrainment 39

5.1 Dimensional Analysis 39

5.2 A General Correlation for the Beginning of Entrainment 41

5.3 Theoretical Considerations for the Beginning of Entrainment in Upward Branches $\quad 42$

5.3.1 Flow Field Generated by the Break 42

5.3.2 Determination of the Beginning of Entrainment 44

5.3.3 Influence of Superimposed Velocities 46

5.4 Results 48

6 Branch Quality and Mass Flux 60

$6.1 \quad$ Single Phase Flow 60

6.2 Two Phase Flow 64

6.2.1 Generalized Representation of Data 64

6.2.2 Experimental Results 64 
6.2 .2 .1 Downward Branch

6.2.2.2 Upward Branch

6.2.2.3 Horizontal Branch

6.2.3 Correlations for the Branch Quality

6.2.3.1 Horizontal Branch

6.2 .3 .2 Downward Branch

6.2 .3 .3 Upward Branch

6.2.4 Correlations for the Branch Mass F1ux

Critical Branch Mass Flux

7.1 The Homogeneous Equilibrium Model (HEM) 86

7.2 Quality and Critical Mass Flux in Horizontal Branches 88

7.3 Applications of the Mode1 88

$\begin{array}{lll}7.4 & \text { Comparison with other Models } & 90\end{array}$

Summary $\quad 94$

9

Literature

Al Method Used to Calculate Propagated Errors

A2 Theoretical Research on the F1ow of Non-homogeneous

Fluids (from A. Craya /12/)

A3 The Homogeneous Equilibrium Model (HEM) (from Moody /35/) 107

A4 RELAP 5 Model for Horizontal Branch Quality

(from Ransom et al. /39/)

A5 Tables of Experimental Data 


\section{Nomenclature}

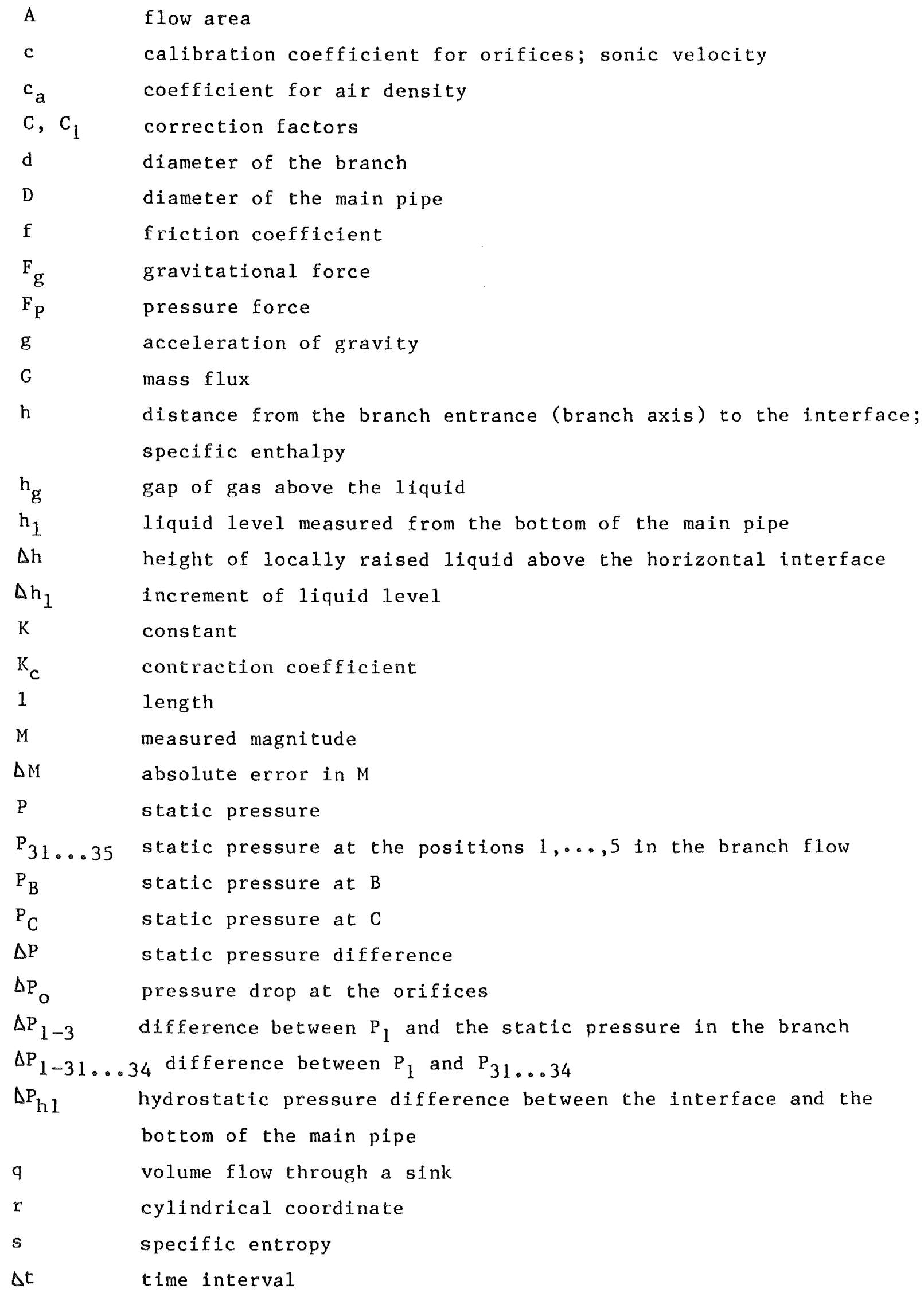




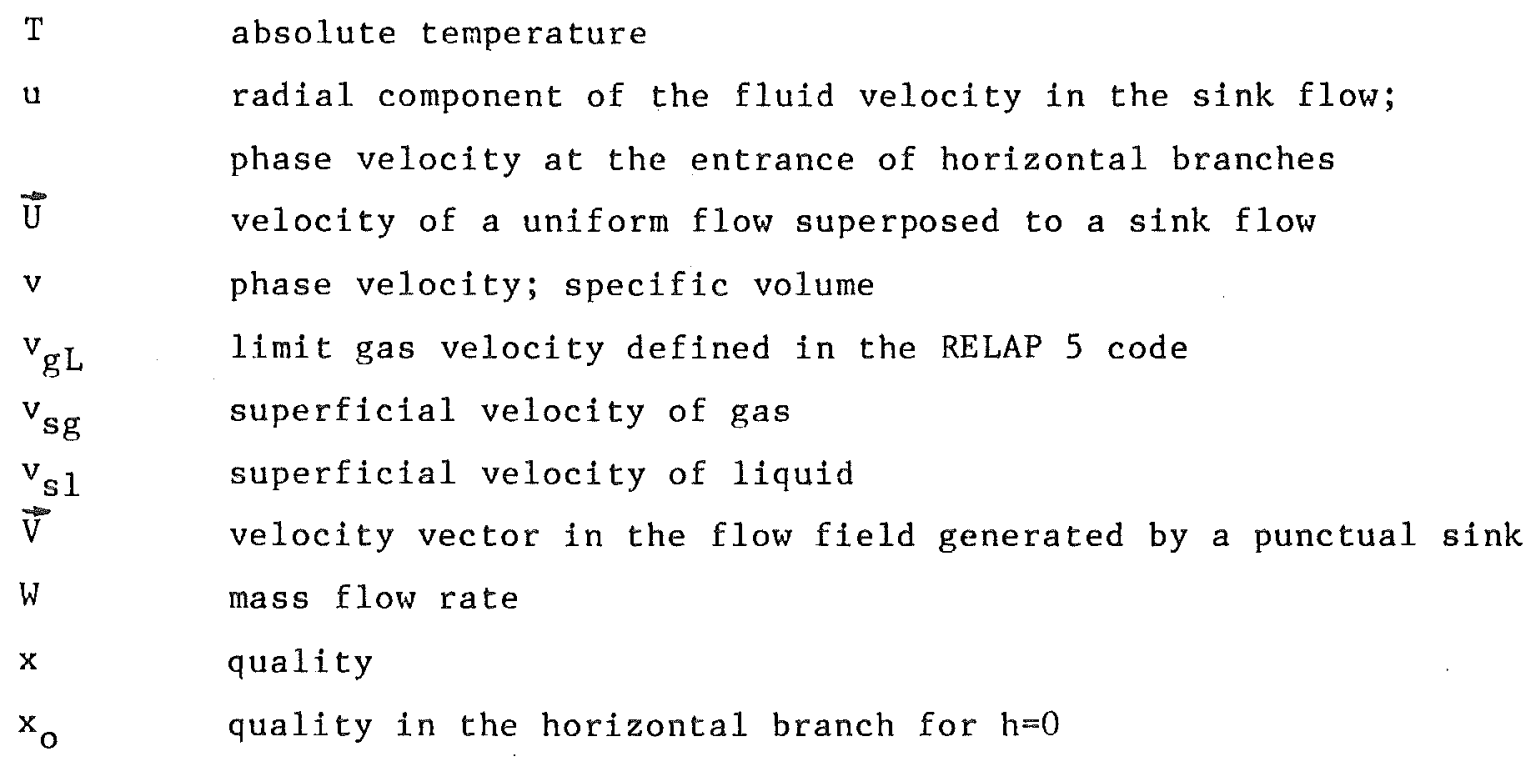

\section{Greek Symbols}

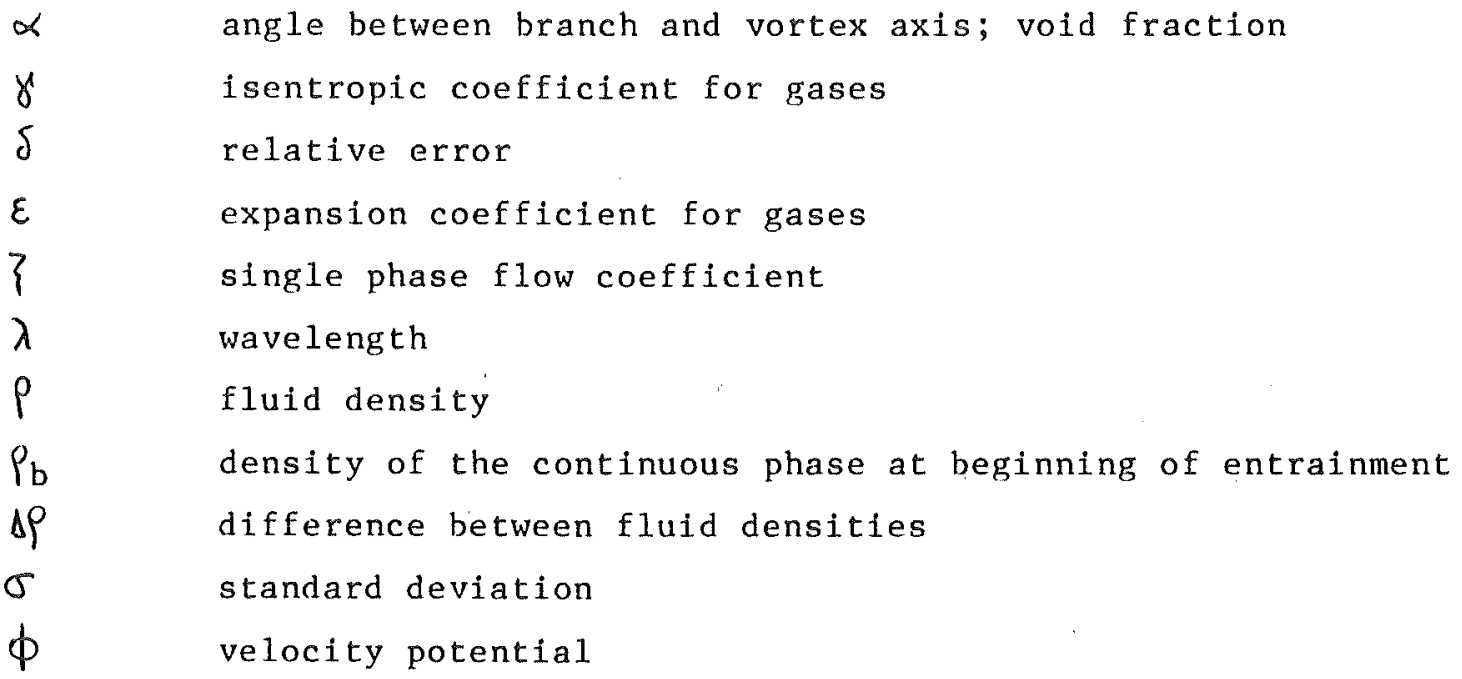

\section{Subscripts}

$\begin{array}{ll}\text { - } & \text { upstream of an orifice; stagnation value } \\ 1 & \text { upstream of the branch in the main pipe } \\ 3 & \text { downstream of the branch in the main pipe } \\ \text { acc } & \text { in the branch } \\ \text { b } & \text { atceleration } \\ \text { crit } & \text { critical } \\ \text { g } & \text { gas } \\ \text { ge } & \text { gas entrainment }\end{array}$




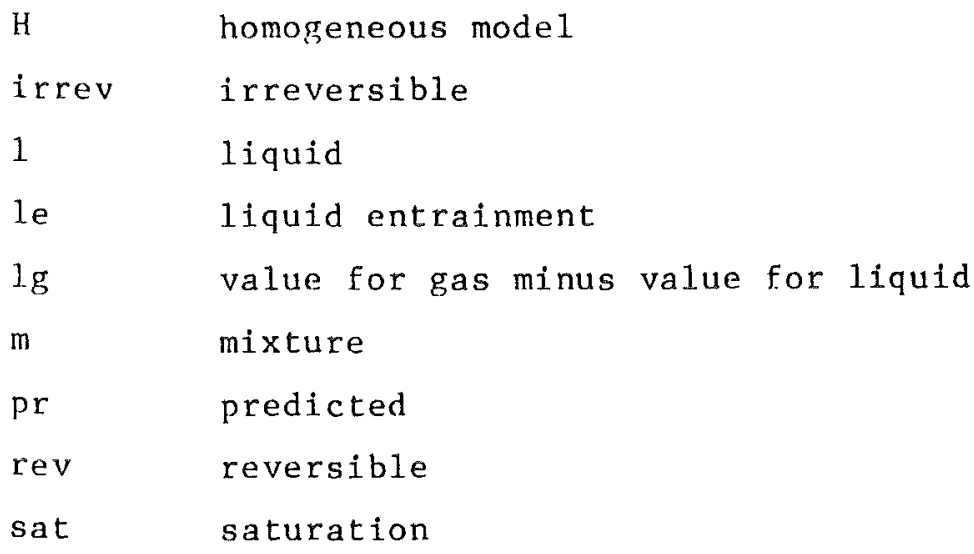

\section{Abbreviations}

b.e. beginning of entrainment

b.g.e. beginning of gas entrainment

b.1.e. beginning of liquid entrainment

g.e. gas entrainment

1.e. liquid entrainment 



\section{Introduction}

The knowledge of two-phase flow in pipe junctions is in general very limited although there are many technical applications. This is due to the complex influence of the phase and velocity distributions and geometrical parameters on the mass and quality redistribution.

Several articles were published in which methods are attempted to determine how two-phase flows split in branching conduits:

Walley and Azzopardi / / and Henry /2/ investigated the case of air-water annular flow in horizontal or vertical T-junctions. Honan and Lahey $/ 3 /$ and Zetzmann $/ 4 /$ performed air-water experiments with different flow regimes in vertical pipes with branches at different angles. Saba and Lahey $/ 5 /$ performed air-water experiments with the same test section as used in $/ 3 /$ but a horizontal main pipe. Reimann et al, /6, $7 /$ and Seeger /8/ made experiments with different air-water and steam-water flow regimes to investigate the phase redistribution in horizontal T-junctions with different branch orientations. In the articles referred to above no stratified flow was investigated and the diameter ratio at the $T$-junction was always $\geq 0.2$.

The special case of branches with a much smaller diameter than the diameter of the horizontal main pipe in which stratified flow prevails will be discussed in the following paragraphs. This situation is of interest in nuclear reactor safety with regard to Loss of Coolant Accidents (LOCAs) caused by small breaks. Here the break mass and enthalpy fluxes determine the behavior of such accidents; these quantities are strongly dependent on the position of the break. If it is located above the horizontal interface, either only vapor will flow out or also liquid will be entrained due to the pressure drop produced by vapor acceleration in the vicinity of the break (hereinafter called the Bernoulli effect). Similarly, with a break located below the interface, vapor may reach the break because of vortex formation or may be pulled-through in a vortex-free flow.

In connection with this problem, liquid removal from large reservoirs through downward, upward or sideward orientated outlets was previously investigated. However, the publications on this subject concentrate on the beginning of entrainment and give no results for the amount of entrainment when the pressure difference through the break is further enlarged or the distance between 
break and interface level is further decreased.

Dagget and Keulegan /9/, for instance, presented data and correlations for predicting incipient vapor entrainment due to a vortex reaching the drain at the bottom of a vessel. The flow entered the test section horizontally, however, a set of vanes at specific angles was used to provide the desired circulation. Lubin and Hurwitz/10/ studied the formation of a dip on the surface of an initially stationary liquid draining through a circular orifice at the bottom of a cylindrical tank. Rouse et al. /11/ investigated the onset of a water spout formed below the intake of a vertical pipe at a certain distance above a horizontal interface between two fluids of different densities. Craya /12/ derived analytically and Gariel /13/ verified experimentally incipient liquid entrainment through side orifices in case of two-layer systems with a horizontal intake above the interface between two incompressible fluids.

The results from these publications were discussed by Zuber /14/ who pointed out that they do not correspond to nuclear reactor conditions. Fig. 1 (from /14/) illustrates the flow mechanisms of interest in nuclear reactors. These arrangements differ significantly from the situations mentioned above mainly due to:

- a flow geometry without rotational symmetry

- the existence of a remaining flow downstream of the break, which will be referred to as a "superimposed velocity" to indicate that there is a net fluid velocity perpendicular to the break flow.

Zuber indicated that currently used thermal hydraulic computer codes cannot predict satisfactorily either the amount of liquid or gas entrainment or the beginning of entrainment. Houdayer et al. /15/ also pointed out the necessity of performing experiments for typical PWR geometries, to be incorporated in the CATHARE code for calculating small or large break LOCA.

Stimulated by Zuber's statements, some investigations were initiated where besides the beginning of entrainment, also the quality and mass-flux through the break were studied:

Crowley and Rothe $/ 16 /$ performed a few air-water experiments at a system pressure of $0.3 \mathrm{MPa}$ with a horizontal $66.6 \mathrm{~mm}$ I.D. pipe and a $6.3 \mathrm{~mm}$ ID side orifice. The afflux was symmetrical from both ends of the pipe. 

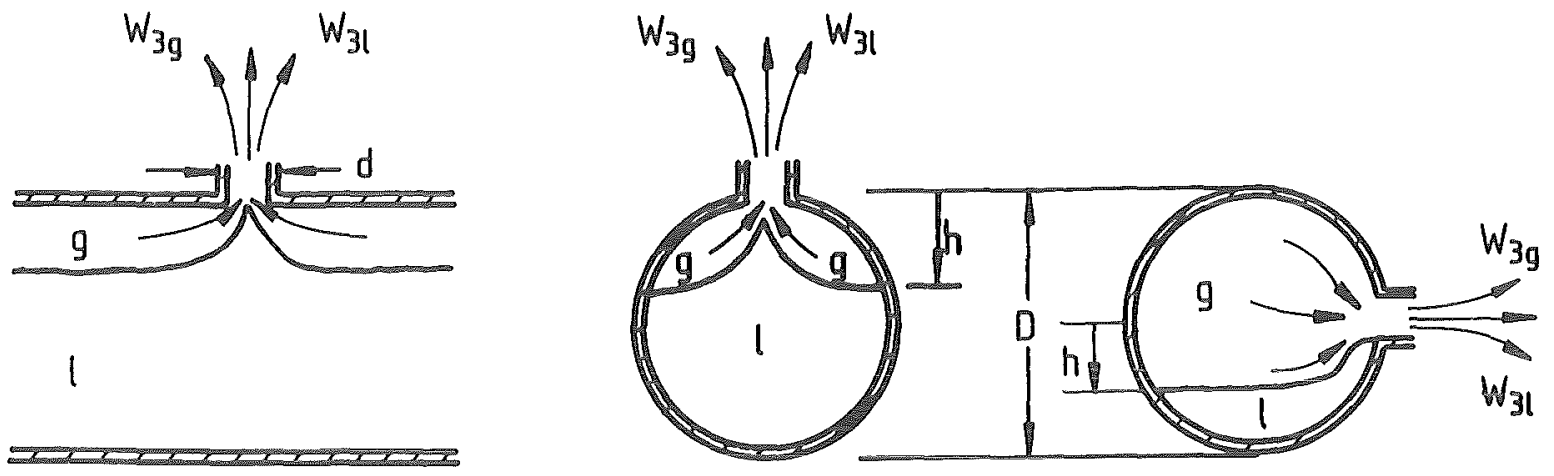

Liquid entrainment due to Bernoulli effect.
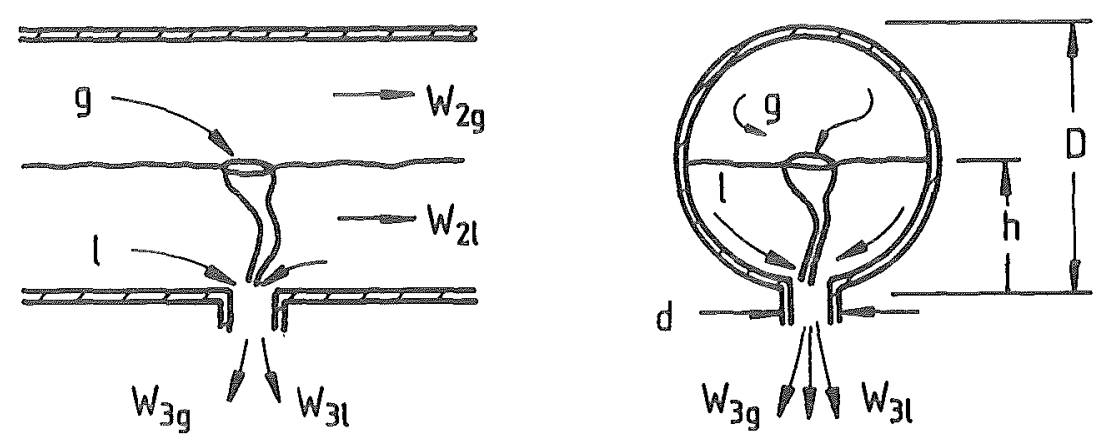

Gas entrainment due to vortex formation.
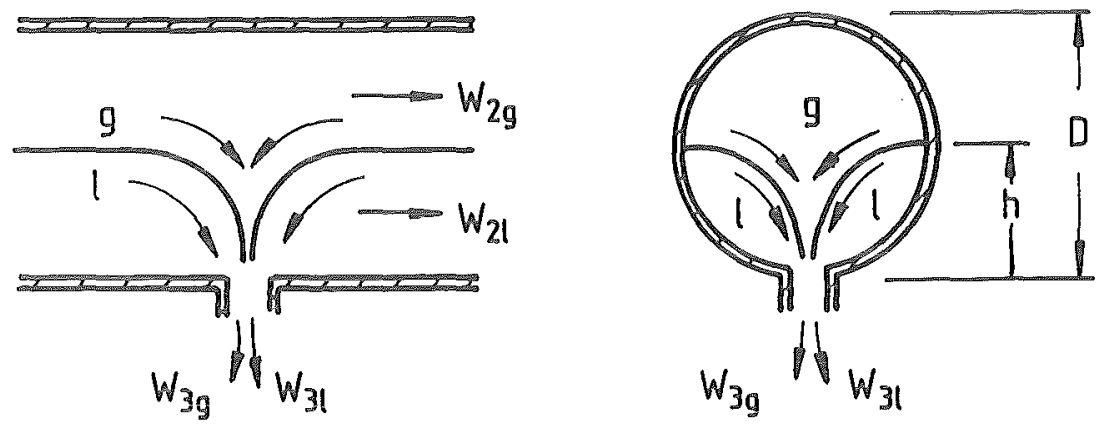

Gas entrainment in vortex free flow.

Fig. 1: Illustration of mechanisms of liquid and gas entrainment in small breaks (trom Zuber /14/). 
Reimann and Khan /17, 18/ simulated the breaks with T-junctions having a ratio of branch to main pipe diameter $d / D<<1$. The authors investigated the air-water flow through a downward orientated branch $(\mathrm{d}=6,12$ and $20 \mathrm{~mm})$ for different flow conditions in a horizontal pipe with $\mathrm{D}=206 \mathrm{~mm}$.

The present work was carried out as a continuation of the investigation initiated by Reiman and Khan: using the same experimental facilities, additional measurements were made with downward branches and further experiments were performed with upward and sideward branches. This report contains:

- The description of the experiments and flow phenomena.

- A general correlation for the beginning of gas and liquid entrainment.

- A model to predict the branch quality and mass flux.

- Extrapolated results for steam-water flows at high pressures. 


\section{Experimental Setup}

The test facility was the same as used in the previous experiments /17, 18/; Fig. 2 shows a scheme of the air-water loop; details are presented in $/ 19 /$. The air is supplied to the loop by a system consisting of four piston compressors followed by an air cooler, an air filter, a spring tube manometer and a mercury thermometer. Cold water is supplied from a reservoir with a circulating pump equipped with a speed controlled D.C. motor. The water temperature and absolute pressure can be measured at the exit of the pump. For steady-state conditions at a maximum system pressure of 11 bar, the aircompressors and water-pump provide maximum mass flow rates of $1 \mathrm{~kg} / \mathrm{s}$ and 30 $\mathrm{kg} / \mathrm{s}$ respectively. As shown by the digram in Fig. 2, two measurement sections of NW 100 and NW 50, respectively, exist for each phase. The gas and liquid mass flow rates can be determined with one orifice in the NW 100 sections and three exchangeable orifices with different mass flow rates but the same $\Delta P$ range in the NW 50 sections. The pressure upstream of the orifices is measured with spring tube manometers and the pressure drop through the orifices is determined with $0-1400 \mathrm{~mm}$ mercury-U-manometers. Downstream of the test section the air-water mixture is carried into a cyclon and, subsequently, the separated air is released to the outside and the water is transferred into the reservoir which feeds the pump.

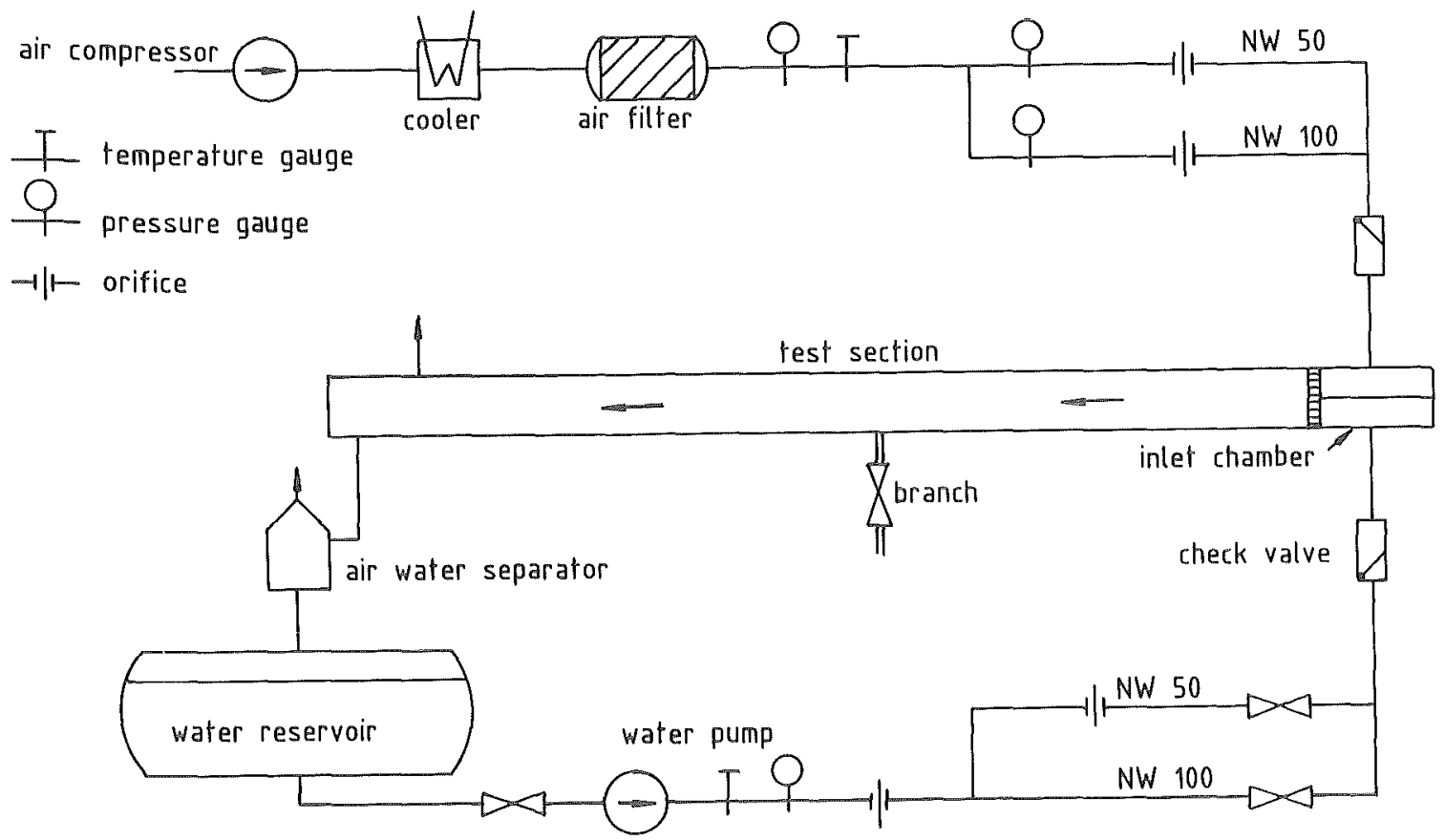

Fig. 2: Two-phase flow air-water loop. 
Fig. 3 shows schematically the test section; the inlet, run and branch variables are characterized by the indices 1,2 and 3 respectively. Metered air and water flows ( $W_{1 g}, W_{11}$ ) enter the horizontal pipe of $206 \mathrm{~mm}$ ID and a total length of about $6 \mathrm{~m}$. The inlet is provided with a chamber specially designed to favor stratified flow (see photograph in Fig. 4a).

A short transparent section of the pipe, made of plexiglass and located about $4.5 \mathrm{~m}$ downstream of the inlet, can be connected to branches with different diameters and orientations. Downstream of the branch a $50 \mathrm{~mm}$ ID pipe with a throttle valve (V3) leads to a separator or directly to a water tank for weight measurements to determine the branch liquid mass flow rate $W_{31}$. The branch gas mass flow rate $W_{3 g}$ can be measured at the exit of the separator with the orifice preceding the valve $v 3 g$.

About $1 \mathrm{~m}$ dornstream of the plexiglass section a movable weir is used to change the liquid level in the test section independently of the rate of liquid flow entering the horizontal pipe. The end of the test section can be removed (Section A-A in Fig. 3) to observe the flow field in the direction of the pipe axis, through a window provided in the flange closing the pipe.

At the exit of the test section the mixture can be throttled to atmospheric pressure through the valve V2. To prevent pressure oscillations which occur when a slug-type two-phase flow is throttled, it is important to assure that the flow leaves the test section homogeneously mixed. For that purpose a . special homogenizer preceding the valve $\mathrm{V} 2$ is used. The dispositive consists essentially of 60 vertical short pipes fixed to a plate as shown in Fig. 4b. Each pipe has two longitudinal slots which allow the two phase flow to fill all the pipes simultaneously. The liquid accumulates at the bottom of the bundle whereas the gas fills the upper portion above the liquid. Independent on the interface level, gas and liquid flow homogeneously mixed through each pipe and therefore the total mixture flows homogeneously.

When only water flows through the valve $V 2$, the orifice preceding the valve $\mathrm{V} 2 \mathrm{~g}$ is used to measure the gas mass flow rate $W_{2 g}$ at the end of the test section. 


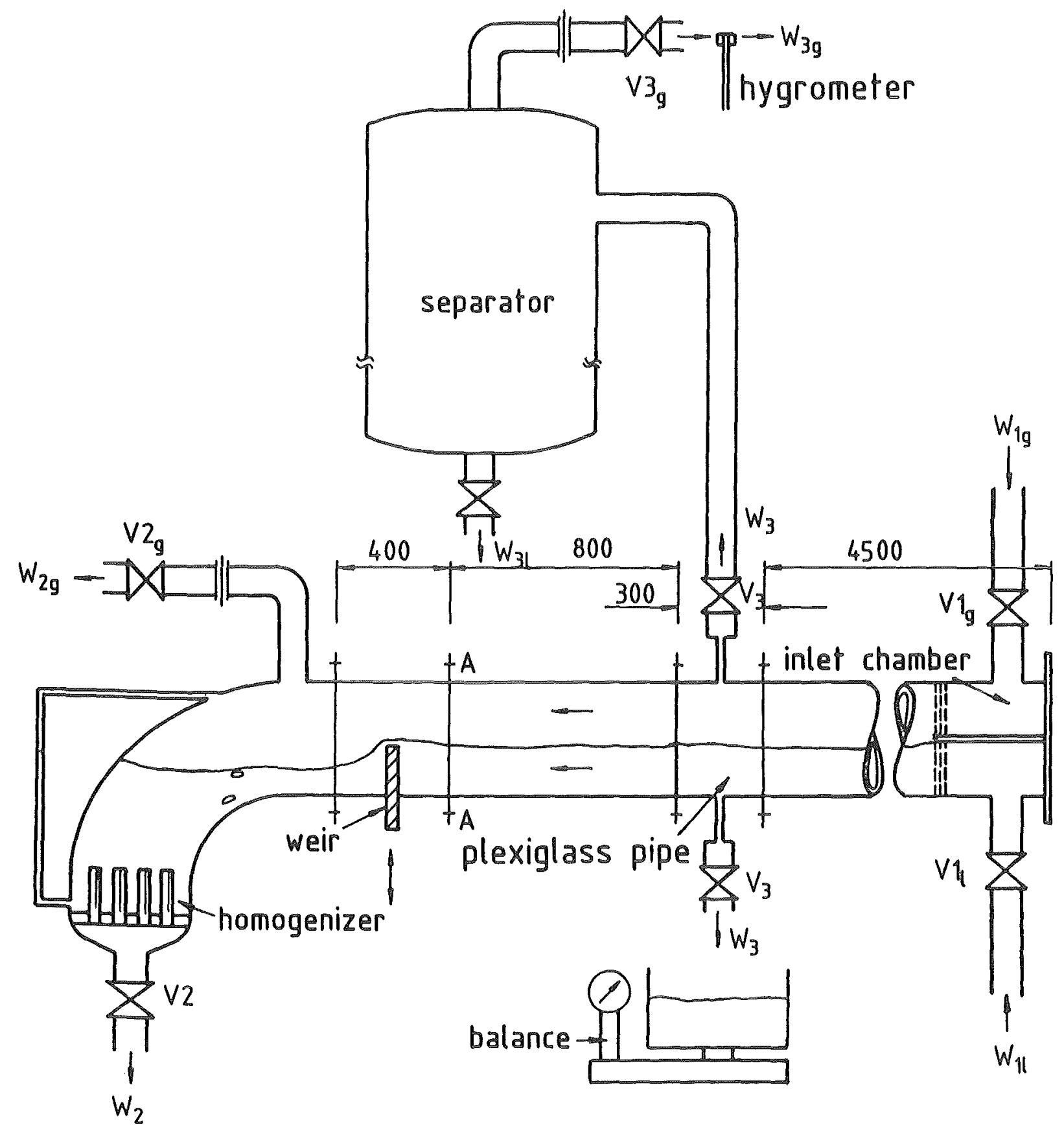

Fig. 3: Test section used for general measurements. 


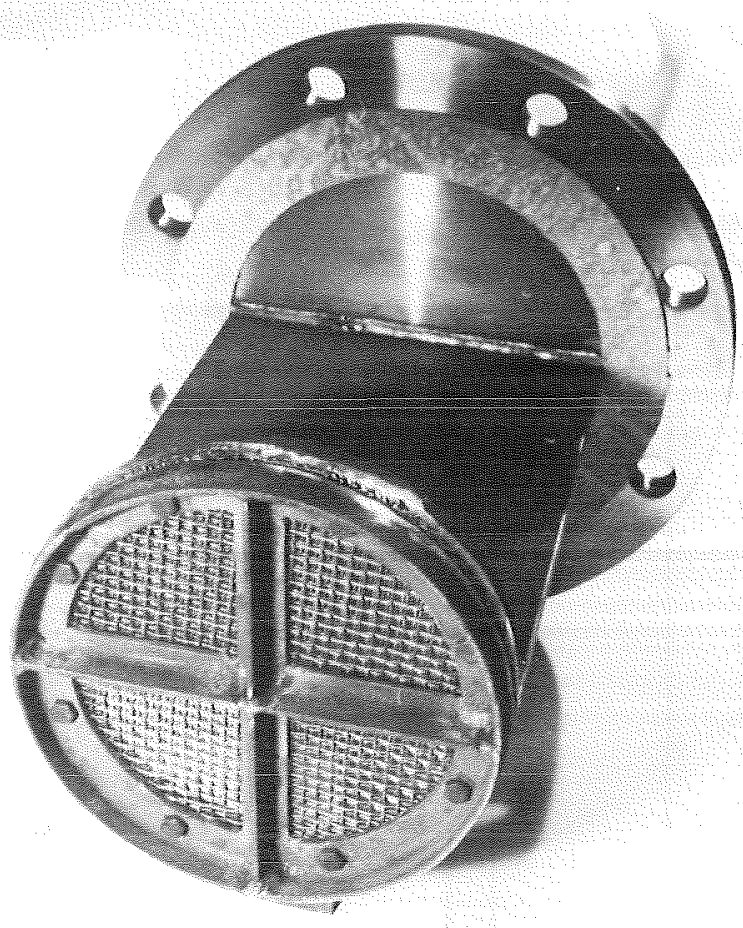

a) Inlet Chamber

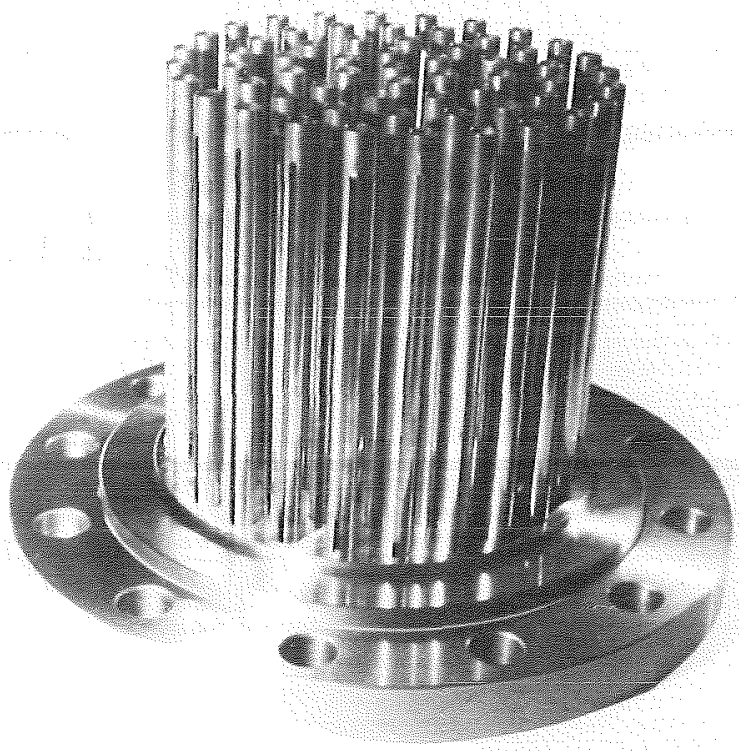

b) Homogenizer

Fig. 4: Details of the test section for small break experiments.

Fig. 5 shows details of the plexiglass pipe. This transparent section contains the branch inserts and allows visual observation of the flow phenomena in the vicinity of the branch entrance. In the main pipe, about $0.5 \mathrm{~m}$ upstream of the T-junction, a pressure tap is connected with the absolute pressure transducer $P_{1}$ and with differential pressure transducers for measurements of the liquid level $\Delta \mathrm{P}_{\mathrm{h} 1}$ and branch pressure drops $\Delta \mathrm{P}_{1-3^{\circ}}$ The 1iquid 1eve1 $\mathrm{h}_{1}$, is also given by a vertical scale downstream of the branch in the plexiglass section.

The branches having a length of $55 \mathrm{~mm}$ and an ID of 6,8, 12 or $20 \mathrm{~mm}$, are pipe studs machined in interchangeable inserts with a sharp-edged entrance. Details are presented in Fig. 6. Four pressure taps at different positions along the branch axis are connected with absolute spring manometers ( $\mathrm{P}_{31}$, $\mathrm{P}_{32}, \mathrm{P}_{33}, \mathrm{P}_{34}$ ) and with differential pressure transducers to measure the pressure differences $\Delta \mathrm{P}_{1-31}, \Delta \mathrm{P}_{1-32}$, etc. Just downstream of the branch, the $50 \mathrm{~mm}$ ID pipe contains a tap for additional pressure measurements $\left(\mathrm{P}_{35}\right)$. 

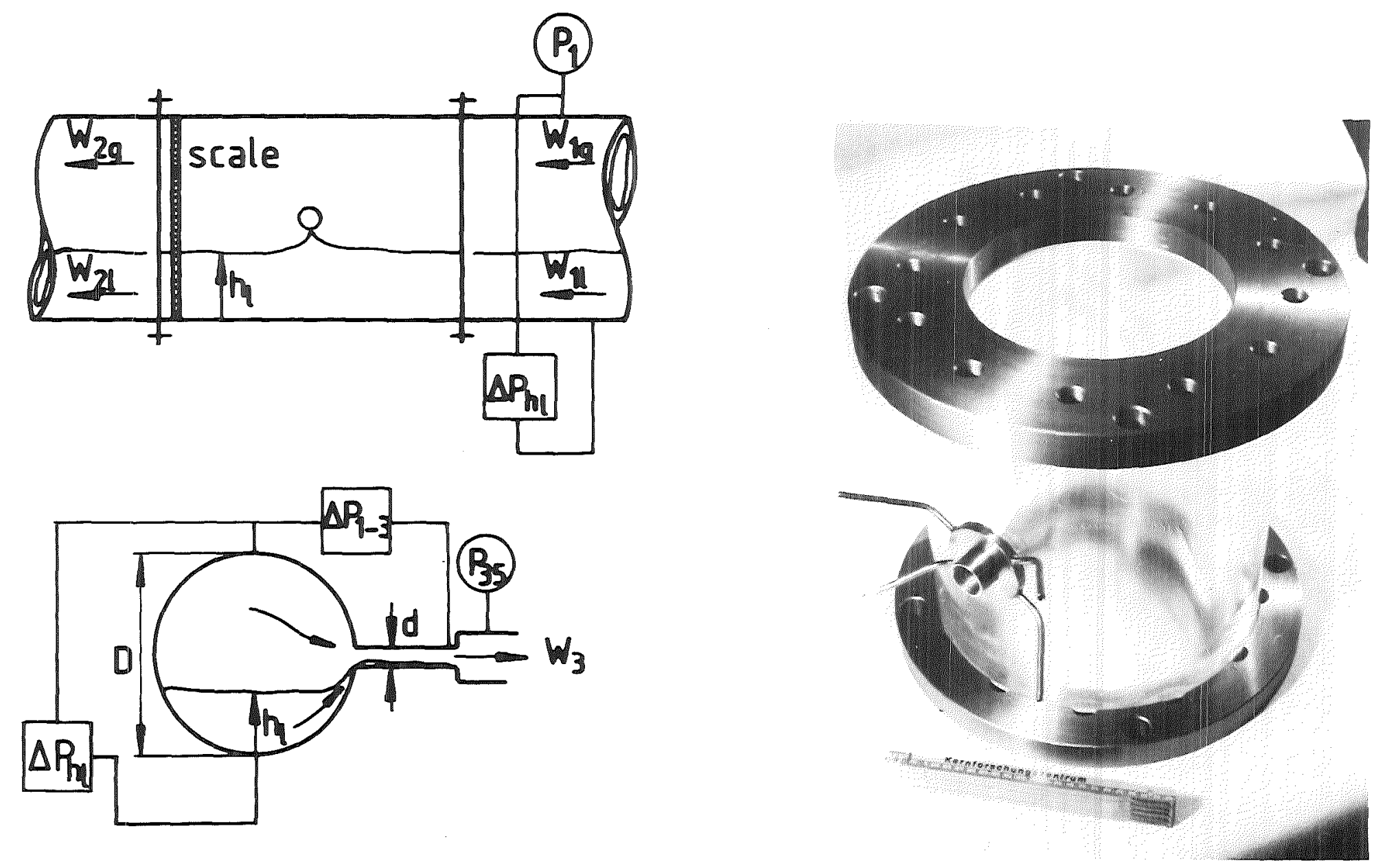

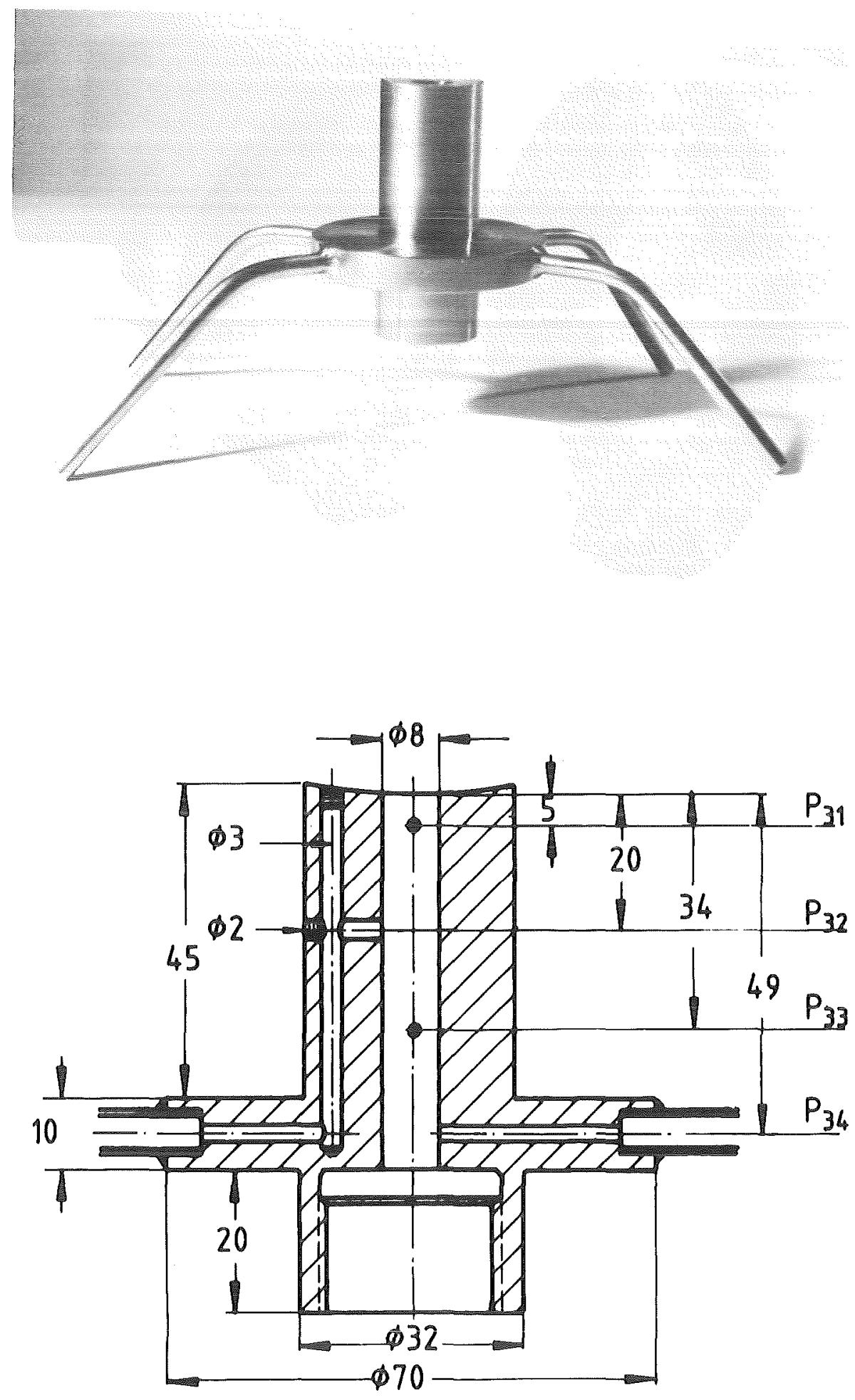

Fig. 6: Photo and details of the pipe studs used to simulate the breaks (Branches). 


\section{Description of the Experiments}

\subsection{General Characteristics}

\subsubsection{Experimental Method}

The experiments were performed with stratified co-current air-water flow in the horizontal main pipe with different interface levels; the system was at ambient temperature and at a maximum pressure of $0.5 \mathrm{MPa}$. The differential pressure $\Delta \mathrm{P}_{1-34}$ was varied between 0.01 and $0.4 \mathrm{MPa}$ by means of the throttle valve $V 3$ downstream of the branch. By continuously decreasing the absolute pressure $P_{35}$, the critical branch mass flux was evidenced by a constant reading of the pressure differences $\Delta \mathrm{P}_{1-3^{\circ}}$. Most of the experiments were performed with subcritical branch mass-fluxes. The fluctuations of $\Delta \mathrm{P}_{1-34}$ increased drastically when entrainment began; therefore, the corresponding signals from the printout of the differential pressure transducer were used, in addition to visual observations, to determine the beginning of entrainment. For quantitative measurements an integrated reading from a voltmeter was used.

The flow field in the vicinity of the branch entrance was observed visually through the plexiglass test section, or through the window at the end of the horizontal pipe when a liquid film on the plexiglass walls made it impossible to observe details of the flow pattern.

During the experiments the interface level $h_{1}$ was fixed by regulating the liquid inflow and outflow and, when the measurement of $\mathrm{W}_{2 \mathrm{~g}}$ was of interest, the interface level at the end of the test section was controlled visually with a transparent $\mathrm{U}$-tube and kept constant at such a position that no bubbles were pulled through valve $\mathrm{V} 2$.

\begin{tabular}{|l|c|c|c|c|c|c|}
\hline \multirow{2}{*}{$\begin{array}{l}\text { Branch } \\
\text { Orientation }\end{array}$} & \multicolumn{7}{|c|}{ Superficial velocities } \\
\cline { 2 - 7 } & $\begin{array}{c}\mathrm{v}_{1 \mathrm{sg}} \\
\mathrm{m} / \mathrm{s}\end{array}$ & $\begin{array}{c}\mathrm{v}_{2 \mathrm{sg}} \\
\mathrm{m} / \mathrm{s}\end{array}$ & $\begin{array}{c}\mathrm{v}_{3 \mathrm{sg}} \\
\mathrm{m} / \mathrm{s}\end{array}$ & $\begin{array}{c}\mathrm{v}_{1 \mathrm{~s} 1} \\
\mathrm{~m} / \mathrm{s}\end{array}$ & $\begin{array}{c}\mathrm{v}_{2 \mathrm{~s} 1} \\
\mathrm{~m} / \mathrm{s}\end{array}$ & $\begin{array}{c}\mathrm{v}_{3 \mathrm{~s} 1} \\
\mathrm{~m} / \mathrm{s}\end{array}$ \\
\hline Downward & 0.495 & 0.109 & 84.72 & 0.154 & 0.095 & 24.71 \\
Upward & 0.711 & 0.253 & 155 & 0.087 & 0.087 & 0.035 \\
Horizontal & 1.068 & 1.020 & 169 & 0.334 & 0.328 & 17.0 \\
\hline
\end{tabular}

Table 1: Maximum superficial velocities registered in the test section and branches * Data from $/ 17,18 /$ 。 
The maximum superficial velocities used in the experiments, defined as the maximum ratios of single phase volume flow rates to total cross section, are summarized in the Table above.

\subsubsection{Measurements Accuracy}

The error in the measured pressures, temperature, liquid level and water weight, constitute the main contributions to the error in the variables calculated from the present experiments. The method used to determine the propagated errors is presented in Appendix Al.

The absolute error for the pressure measured with spring tube manometers ranges between +0.05 bar for steady-state single-phase flow and to.2 bar for the very unstable two phase flows occurring in the branches. In these cases, however, the integrated digital reading from the voltmeter used to determine the pressure differences $\Delta \mathrm{P}_{1-31}, \Delta \mathrm{P}_{1-32}$, etc. has a relative error $<0.5 \%$ of the full scale. Therefore, maximal absolute errors of $\pm 0.01, \pm 0.05, \pm 0.1$ and +0.25: bar can be assigned to pressure differences below $0.2,1,2$ and 5 bar, respectively, at the end of the scale. The absolute error in the reading from the $U$-tube mercury manometers range between $\pm 1 \mathrm{~mm} H$ for very stable flow and $\pm 10 \mathrm{~mm} \mathrm{Hg}$ when fluctuations occur。

The error for the absolute temperature $\mathrm{T}$ measured with lercury thermometers is about $\pm 1.5 \mathrm{~K}$.

The rates of single phase gas and liquid mass flow through the orifices were determined with equations of the form: $W_{g}=c_{g}\left(P_{0} \Delta P_{o} / T_{o}\right)^{0.5}$ and $W_{1}=$ $c_{1}\left(P_{1} \Delta P_{0}\right)^{0.5}$, where $c_{g}$ and $c_{1}$ are the orifice calibration coefficients for gas and liquid, respectively, $P_{O}$ and $T_{0}$ are the pressure and temperature upstream of the orifice, and $\Delta \mathrm{P}_{\mathrm{o}}$ is the pressure drop through the orifice. Using these equations with the typical values for the inflow $\mathrm{P}_{0}=7$ bar, $\mathrm{T}_{\mathrm{O}}=$ $15^{\circ} \mathrm{C}$ and $100 \mathrm{~mm} \mathrm{Hg}<\Delta \mathrm{P}_{\mathrm{o}}<1400 \mathrm{~mm} \mathrm{Hg}$, the absolute errors estimated above give relative propagated errors in the ranges $1-6 \%$ for $W_{1 g}$ and $0.4-5 \%$ for $\mathrm{W}_{11}$, the maximum relative errors corresponding to minimum mass flow rates. Similar values are valid for mass flow rates measured with the orifices preceding the valves $\mathrm{V} 2_{\mathrm{g}}$ and $\mathrm{V} 3 \mathrm{~g}^{\circ}$ 
In the range of the present experiments the density of the water was considered as a constant with negligible error and the density of the air in the test section was given by $\rho_{g}=c_{a} P_{1} / T_{1}$ with $c_{a}=348.5 \mathrm{~kg} \mathrm{~K} \mathrm{~m}^{-3}$ bar ${ }^{-1}$, which produces a typical relative error of $\pm 2.5 \%$

For a smooth stratified interface in the main pipe the vertical millimetred scale used to measure liquid levels, allows readings with an absolute error of $\pm 1 \mathrm{~mm}$. In experiments with a wavy interface, an estimated mean value of the liquid levels read on the scale and recorded in the printout of the $\Delta \mathrm{P}_{\mathrm{hl}}$ transducer, was considered. The corresponding absolute error depends on the waves amplitude; maximum estimated values are about $\pm 5 \mathrm{~mm}$.

Measurements of the water weight were made using, a balance with a precision of $\pm 0.1 \mathrm{~kg}$. To measure small quantities of water $(\leq 2$ liters $)$ accumulated in the separator connected to upward branches, a recipient was used with a scale allowing readings with an error of \pm 0.005 liter.

Time intervals were measured using a chronometer with a precision of +0.1 sec. However, an estimated error of about \pm 3 sec. was introduced by indecerminations in the times corresponding to beginning and end of the measured interval.

\subsection{Experiments with Downward Branches}

In addition to previous investifations /17, $18 /$ some experiments were performed to measure the beginning of gas entrainment ( $b_{\circ} g_{\circ} e_{0}$ ) and the gas entrainment (g.e.) without superimposed liquid velocities, that means with V2 closed.

To determine the b.g.e., the input and branch mass flow rates were adiusted in such a way that the interface level decreased very slowly (for instance $\Delta \mathrm{h}_{1}=5 \mathrm{~mm}$ in $100 \mathrm{sec}$ ). The system pressure was kept constant by using a small gas flow regulated with the valves $\mathrm{Vl}_{\mathrm{g}}$ and $\mathrm{V} 2 \mathrm{~g}$. Therefore the experiment can be assumed to be steady-state. The formation of first gas hoses reaching the break outlet and producing a characteristic change in the signal from the $\Delta \mathrm{P}$ transducers, was considered as the b.g.e. and the corresponding liquid level was recorded. 
In experiments to measure the g.e., the valves $\mathrm{V}_{2} \mathrm{~g}$ and $\mathrm{V} 2$ were closed and a balance between the input and break mass flow rates was adjusted to keep the liquid level constant (steady-state experiments). Therefore, the gas and the liquid mass flow rates through the branch were determined with the orifices preceding the main pipe inlet (Fig. 3). In previous experiments with superimposed liquid velocities $/ 17,18 /$, the branch liquid mass flow rate $W_{31}$ was determined by weighing a timed sample at the exit of the branch, and the superimposed mass flow rate $W_{21}$ was the difference $W_{11}-W_{31^{\circ}}$

\subsection{Experiments with Upward Branches}

In this case the beginning of liquid entrainment (b.1.e.) and the liquid entrainment (1.e.) for different interface levels in the main pipe were measured. The small quantity of liquid entrained in upward branches is mixed with a relative high gas mass flow rate. Therefore, for quantitative measurements the experiments were performed with the branch connected to the separator as shown in Fig. 3.

A constant $W_{3 g}$ at a constant pressure $P_{1}$ was adjusted to a slowly increasing liquid level $\left(\Delta \mathrm{h}_{1} / \Delta \mathrm{t} \cong 1 \mathrm{~mm} / \mathrm{min}\right)$ and the b.l.e. was determined visually when a few droplets raised from the interface reached the break outlet. Suitable orifices preceding the valve $V 3_{g}$ at the exit of the separator were used to measure $W_{3 g}$ and, in case of superimposed gas velocities given by the difference $W_{1 g}-W_{3 g}$. The highest superimposed gas velocities were limited by the occurrence of high waves or slug flow in the main pipe.

To determine the entrainment rate, it was waited until equilibrium established between liquid inflow and outflow in the test section, and then the water was collected at the bottom of the separator during a defined period of time after which the liquid was measured. In experiments with superimposed liquid velocities the difference $W_{11}-W_{31}$ was used to determine $W_{21}$.

The liquid-gas interaction due to entrainment phenomena, produces an in creased evaporation of water downstream of the branch inlet which leads to underestimation of $W_{31}$. In order to evaluate this amount of water, the humidity of the branch gas flow was measured at the exit of the separator in some experiments with and without liquid entrainment. The correspondig differences were used to determine the mass of evaporated water, which reached values 
between 10 and $20 \%$ of the liquid collected.

\subsection{Experiments with Horizontal Branches}

With horizontal branches both gas and liquid entrainment can occur depending on the interface level in the main pipe.

To determine the b.g.e. and the g.e., the same procedure as described in 3.2 was applied. For the b.g.e. additional, experiments with superimposed gas or liquid velocities were performed. In these cases the branch gas flow rate is negligible; therefore, $W_{2 g}$ was given by the measured $W_{1 g} ; W_{21}$ was the difference $W_{11}-W_{31}$.

To determine the b.1.e. and the 1.e., the method described in 3.3 was followed; however, the separator was not used and the branch was directly connected to the water tank to weigh timed samples.

The b.1.e. started when a very thin film of water raised from the interface moving on the pipe wall and reached the branch entrance. This was observed visually and from a characteristic change in the signal from the $\Delta P$ transducer.

For the b.l.e. again additional experiments with superimposed veloctties were performed. 


\section{Flow Phenomena}

\subsection{Downward Branch}

Although in the present investigations the experiments with downward branches were performed always with V2 closed, the description and photographs of the flow with superimposed liquid velocities (from $/ 17,18 /$ ), will be included to get a complete picture of the flow phenomena.

If liquid is supplied to the test section with the valve V2 closed, the inflow rate is equal to the branch flow rate and no liquid flow perpendicular to the branch axis results. This situation is unstable with regard to the enhancement of velocity components around to the branch axis and causes a vortex flow, Figs. 7-8 (from /17, 18/). The vortex axis is deflected somewhat in the direction opposite to the inflow side and the gas entrainment starts when a very thin gas hose reaches the branch inlet (Fig. 7)。
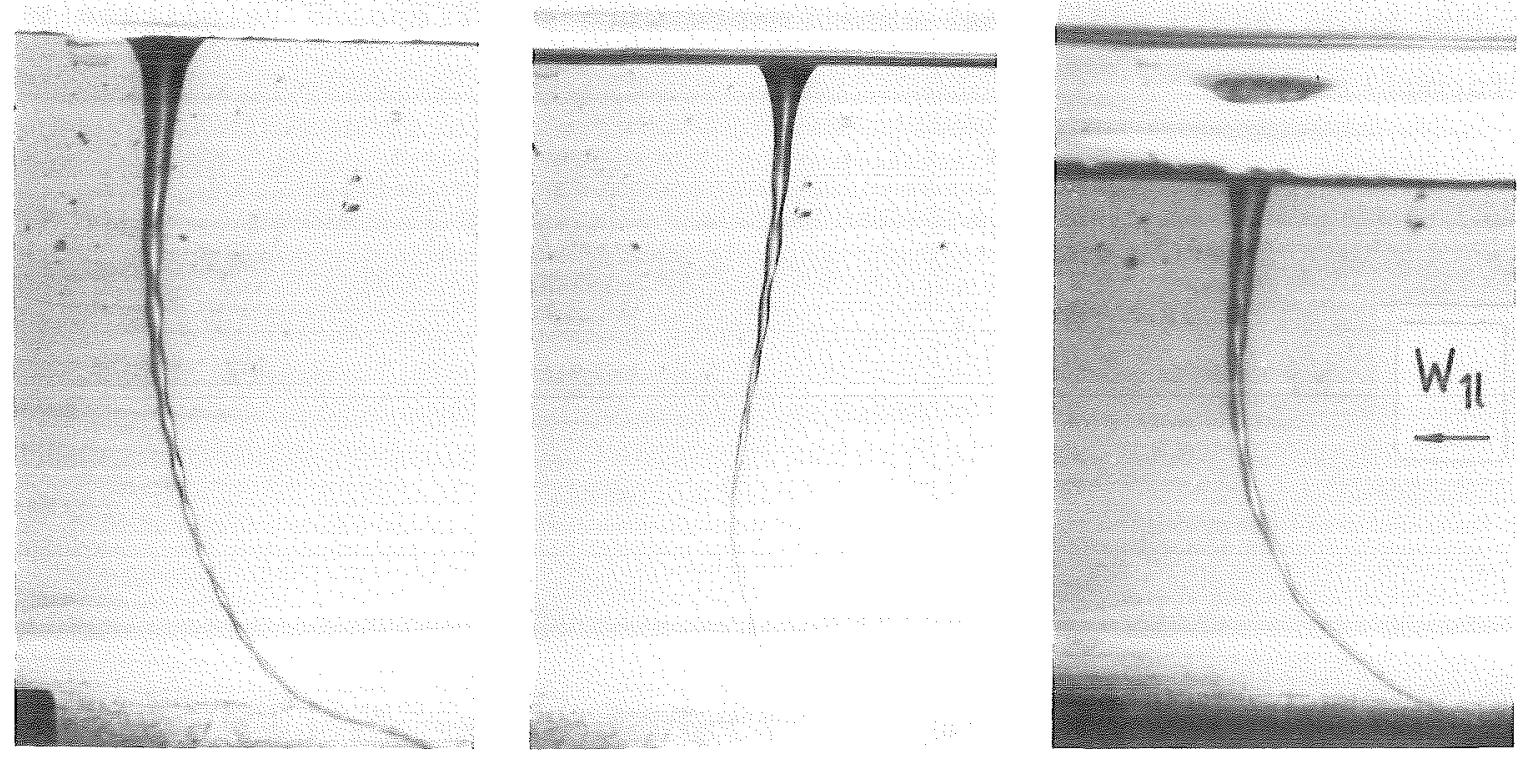

Fig. 7: Beginning of gas entrainment in downward branch (from $/ 17,18 /$ ) 

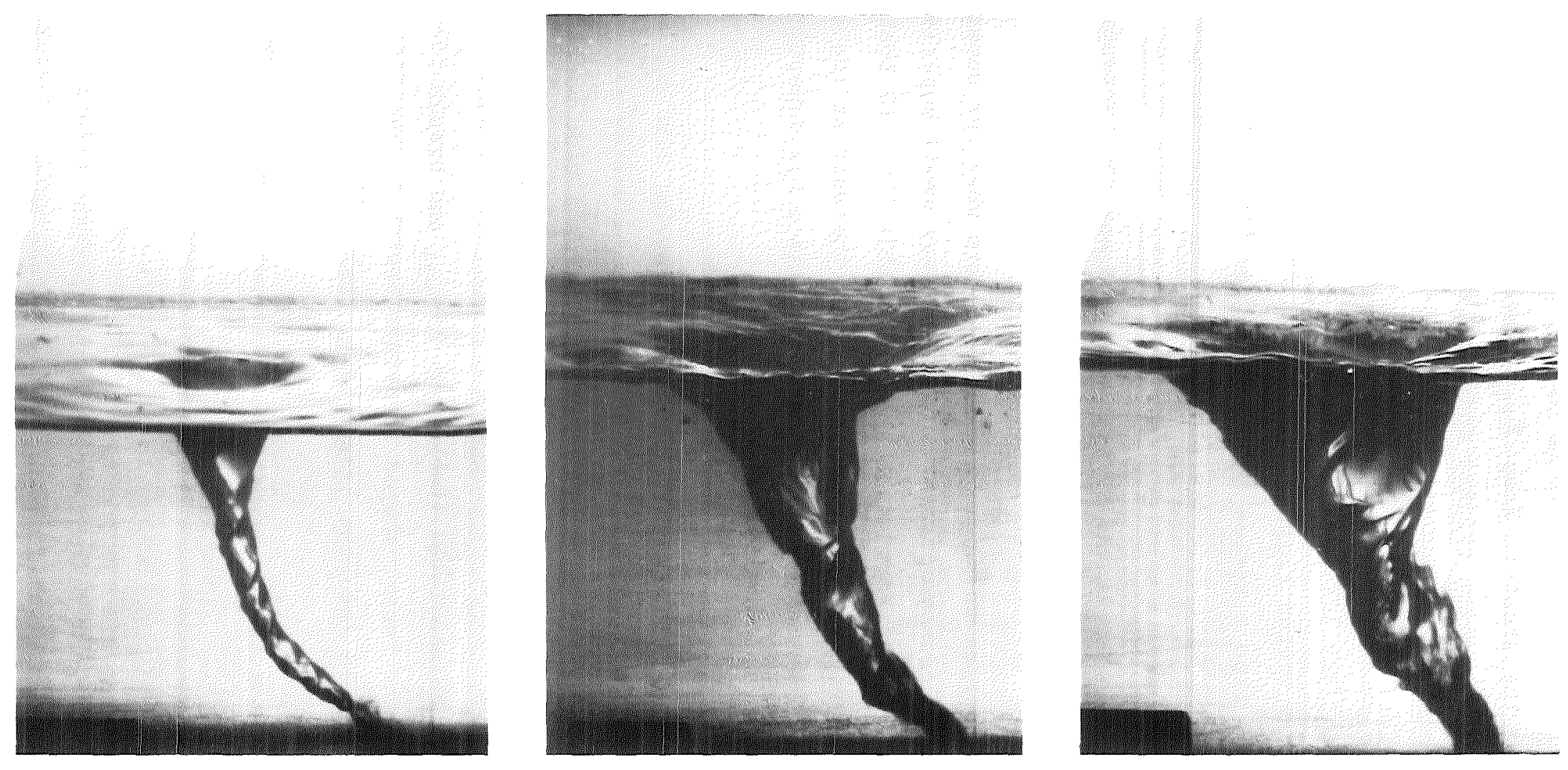

increasing branch mass flow rate

Fig. 8: Development of gas entrainment in downward branch for increasing $W_{3}$; $\mathrm{W}_{21}=0($ from $/ 17,18 /)$. 
This first gas hose is not stable but swept away after some seconds, and a long time can pass until another gas hose is formed. By lowering the interface level or increasing the pressure drop $\Delta p_{1-34}$ and branch mass flow rate, respectively, the gas hose becomes thicker and more stable (Fig. 8).

In Fig. 9.a the spiral-type streamlines at the interface are clearly seen. By lowering further the interface level a condition is reached where the water flow pattern changes from vortex flow to vortex-free flow (Figure 9.b). A reason for the transition from a vortex to a vortex free flow field is the increasing influence of the wall friction with decreasing interface levels. Dagget and Keulegan /9/ found, in similar experiments, that near the bottom of a vessel the radial velocity of the vortex increases and the tangential velocity rapidly decreases due to the development of a boundary layer.

Another transition from vortex to vortex free flow occurs when the superimposed liquid velocity exceeds a certain value. With very small superimposed velocities $\left(V_{21} \leq 0.06 \mathrm{~m} / \mathrm{s}\right)$ the vortices are very unstable (Fig. 10 from $120 /$ ). Such low values of $v_{21}$ were obtained by raising the weir at the end of the test section, which gives a high interface level with a low superimposed velocity.

At higher superimposed velocities with low interface levels, e.g. $v_{21}=0.25$ $\mathrm{m} / \mathrm{s}$, the flow field observed in the experiments was always vortex free as shown in Fig. 11 (from /17, 18/). The photograph 11 a shows a test point without gas entralnment but an already considerably deflected interface.

At a slightly lowered interface level (increased $\Delta \mathrm{P}_{1-34}$ and branch flow, respectively) the tip of the funnel-shaped interface begins to oscillate and gas is intermittently sucked into the branch (Fig. 11b).

At lower interface levels a continuous gas pull-through is reached (Fig. $1 \mathrm{lc}$ )。

\subsection{Upward Branch}

For liquid levels far below the branch entrance and constant values of $\Delta P_{1-34}$ and $W_{3 g}$, only gas enters the branch, the interface being a nearly ideal horizontal plane. Due to the acceleration of the gas entering the 


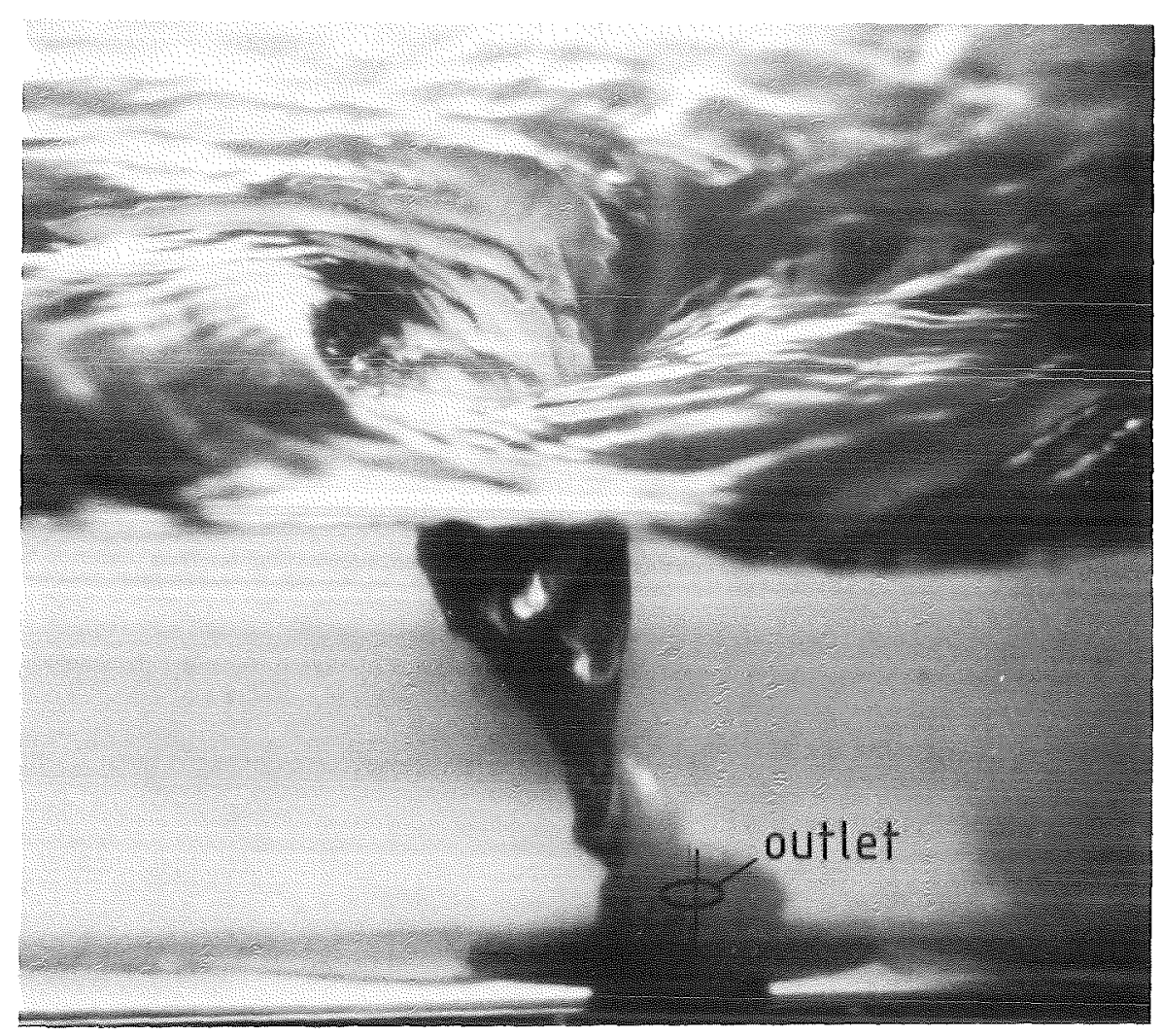

a) strong vorticity at intermediate interface level

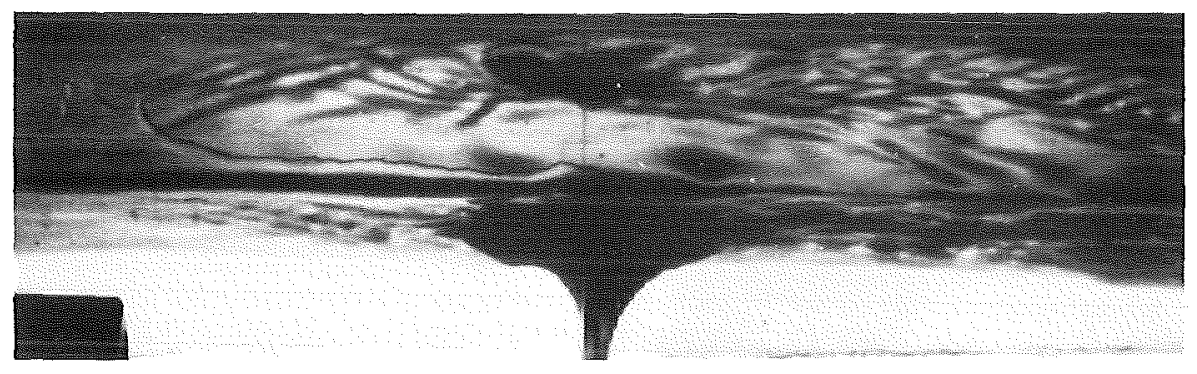

b) vortex free flow at low interface level

Fig. 9: Gas entrainment in downward branch; $W_{21}=0$ (from $\left./ 17,18 /\right)$. 

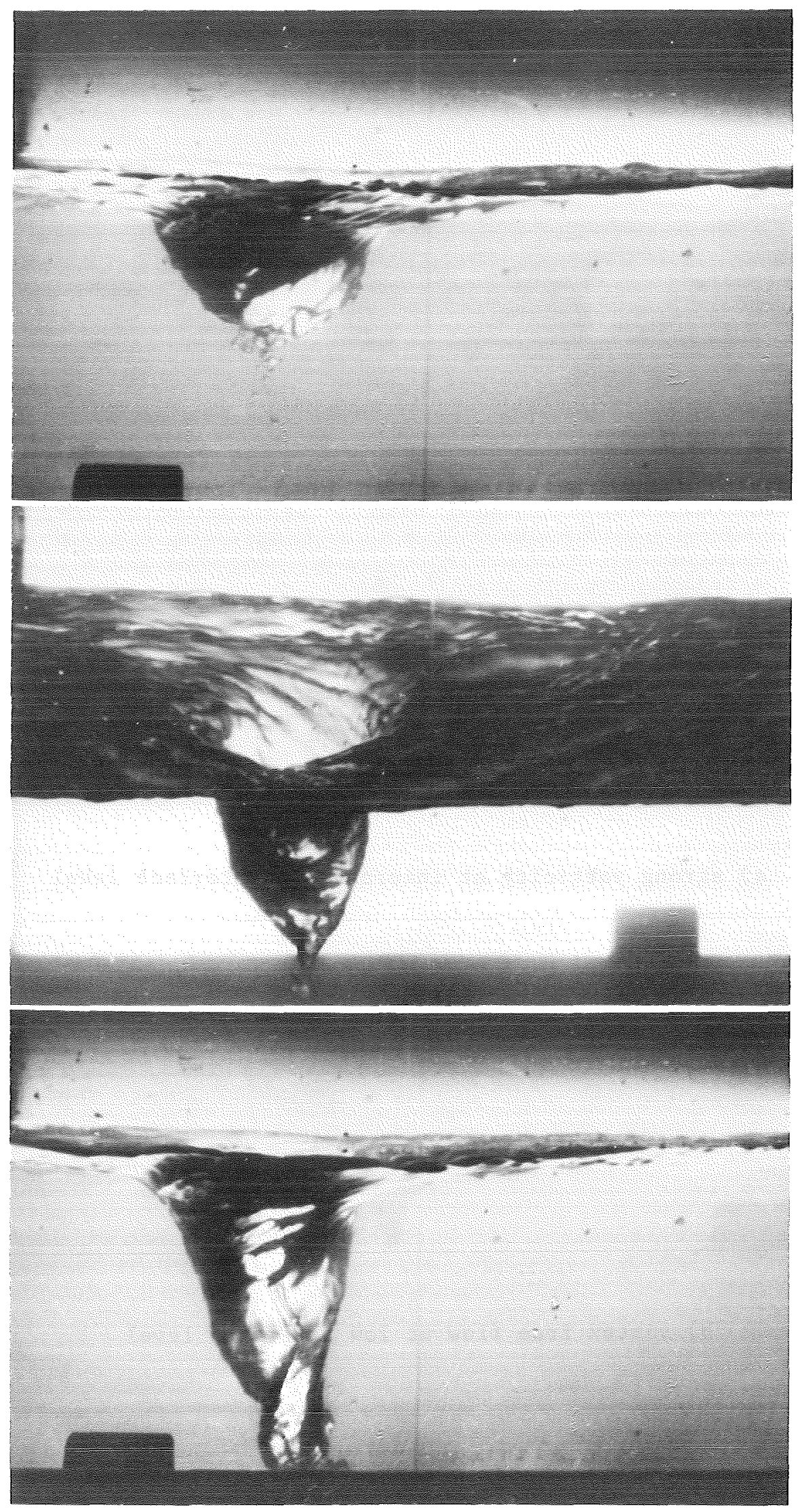

Fig. 10: Unstable vortex gas entrainment in downward branch occurring at high interface level with $v_{21} \quad 0.04 \mathrm{~m} / \mathrm{s}$ (from $/ 20 /$ )。 


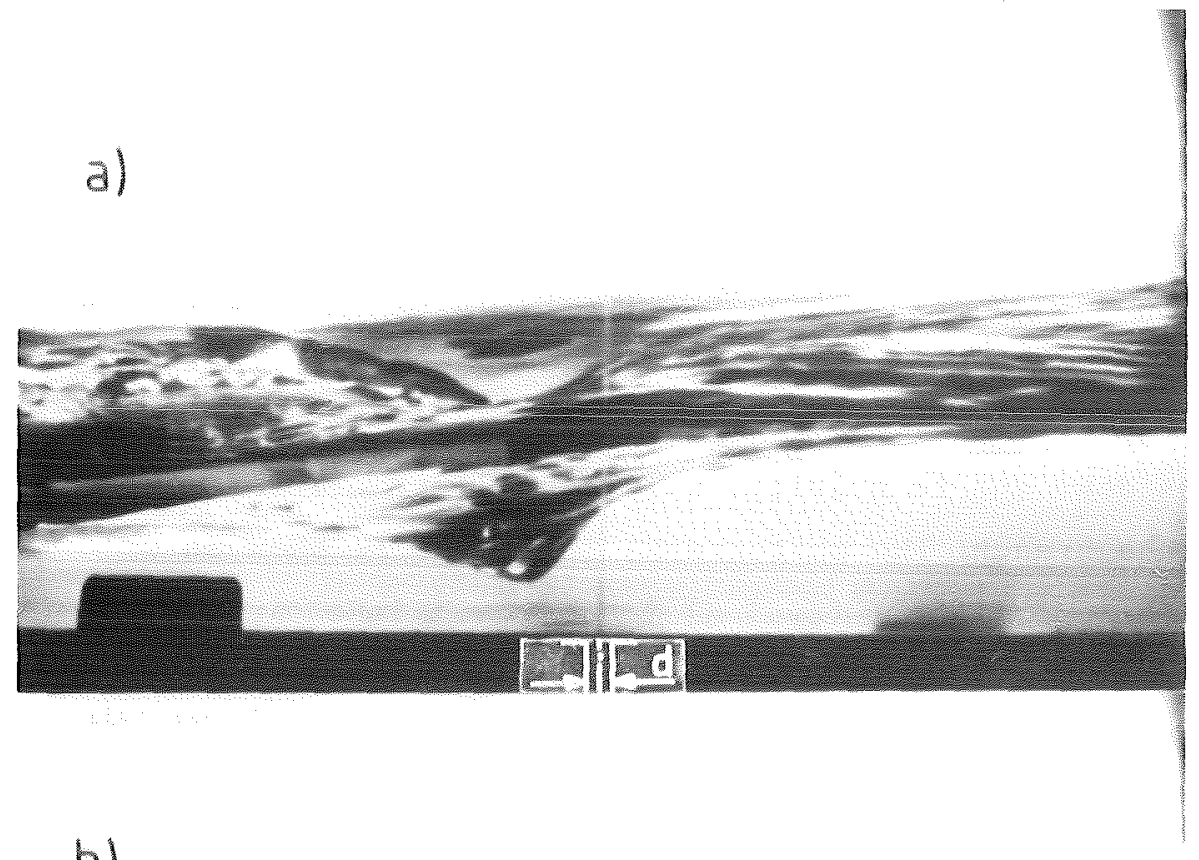

b)

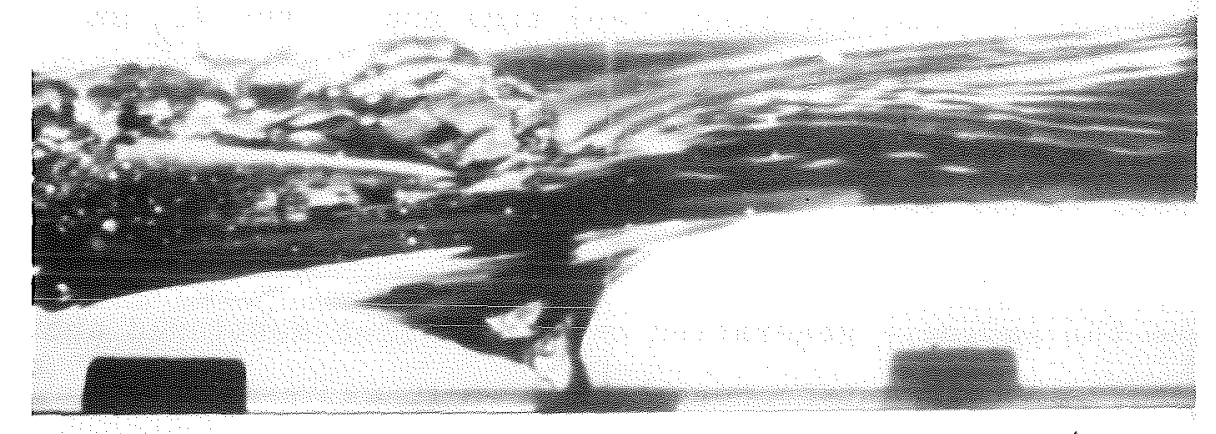

c)

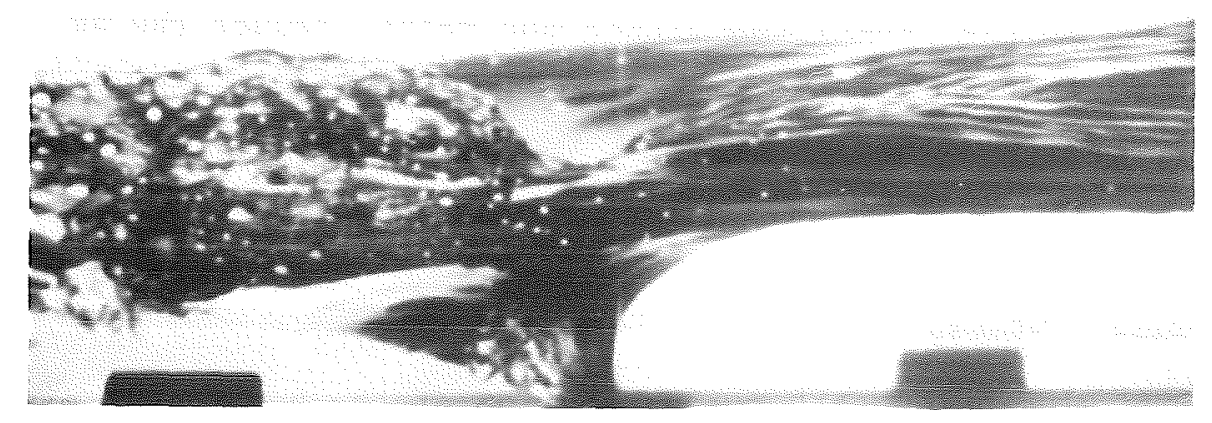

Fig. 11: Development of vortex free gas entrainment in downward branch for $v_{21}=0.3 \mathrm{~m} / \mathrm{s}$ and increasing $\Delta \mathrm{P}_{1-34}($ from $/ 17,18 /)$ :
a) no gas entrainment,
$\Delta \mathrm{P}_{1-34}=0.04 \mathrm{MPa}$;
b) oscillating gas entrainment, $\Delta \mathrm{P}_{1-34}=0.065 \mathrm{MPa}$;
c) continuous gas entrainment, $\Delta \mathrm{P}_{1-34}=0.22 \mathrm{MPa}$. 
branch the pressure above the interface is lowered (Bernoulli effect) and the interface below the branch entrance is locally raised.

Fig. 12 (from /21/) shows sequences of photographs (48 pictures/s) taken from the end of the horizontal pipe. A considerable amount of liquid can be torn away from the interface. However, due to the large vorticity of the gas flow, the droplets are radially accelerated and only a small fraction reaches the branch inlet. The droplets hitting the wall give rise to a liquid film which mostly drains down due to gravity; only a small fraction near the inlet is sucked into the branch. The photographs in Figure 12 show an oscillatory behavior of the entrainment (typical frequency $\simeq 3 \mathrm{~Hz}$ ) probably initiated by a momentarily larger amount of liquid entering the branch which causes a decrease of the gas flux. This decrease of gas flux diminishes the liquid entrainment which again increases the gas flux, etc.

Fig. 13 (from /21/) contains photographs taken through the plexiglass test section. The inflow is from the right-hand side and valves $\mathrm{V} 2 \mathrm{~g}$ and $\mathrm{V} 2$ are closed. In photo 13a the location of the maximum interface deflection (lowest local pressure) is shifted some centimeters to the left side. This asymmetry also contributes to the fact that the liquid portion reaching the branch is small.

In addition to the periodic generation of entrainment mentioned above, another oscillatory entrainment process occurred; It was caused by low amplitude interface waves with wavelengths $\lambda$ of about $15 \mathrm{~cm}$ (Fig. 13b). These are surface gravity waves driven by a balance between the fluid's inertia and its tendency, under gravity, to return to a state of stable equilibrium with the heavier fluid (water) underlying the lighter (air) (see e.g. Lighthi11/22/). Such waves occur at high inlet gas fluxes and rather reduce the amount of liquid entrainment or decrease the distance between branch entrance and interface at the beginning of entrainment. A further increase of the gas inflow rate finally causes the transition from stratified to slug flow by growing low-frequency waves of wavelengths of the order of the pipe diameter. Fig. $13 \mathrm{c}$ shows a test point in the slug flow regime: mainly liquid enters the branch when the slug bridges the cross section of the horizontal pipe, and the time averaged branch liquid flow rate is considerably increased. The occurrence of slug flow is coupled to strong pressure oscillations in the test section, up to several bars in the present experiments. This flow regime was therefore generally avoided. 

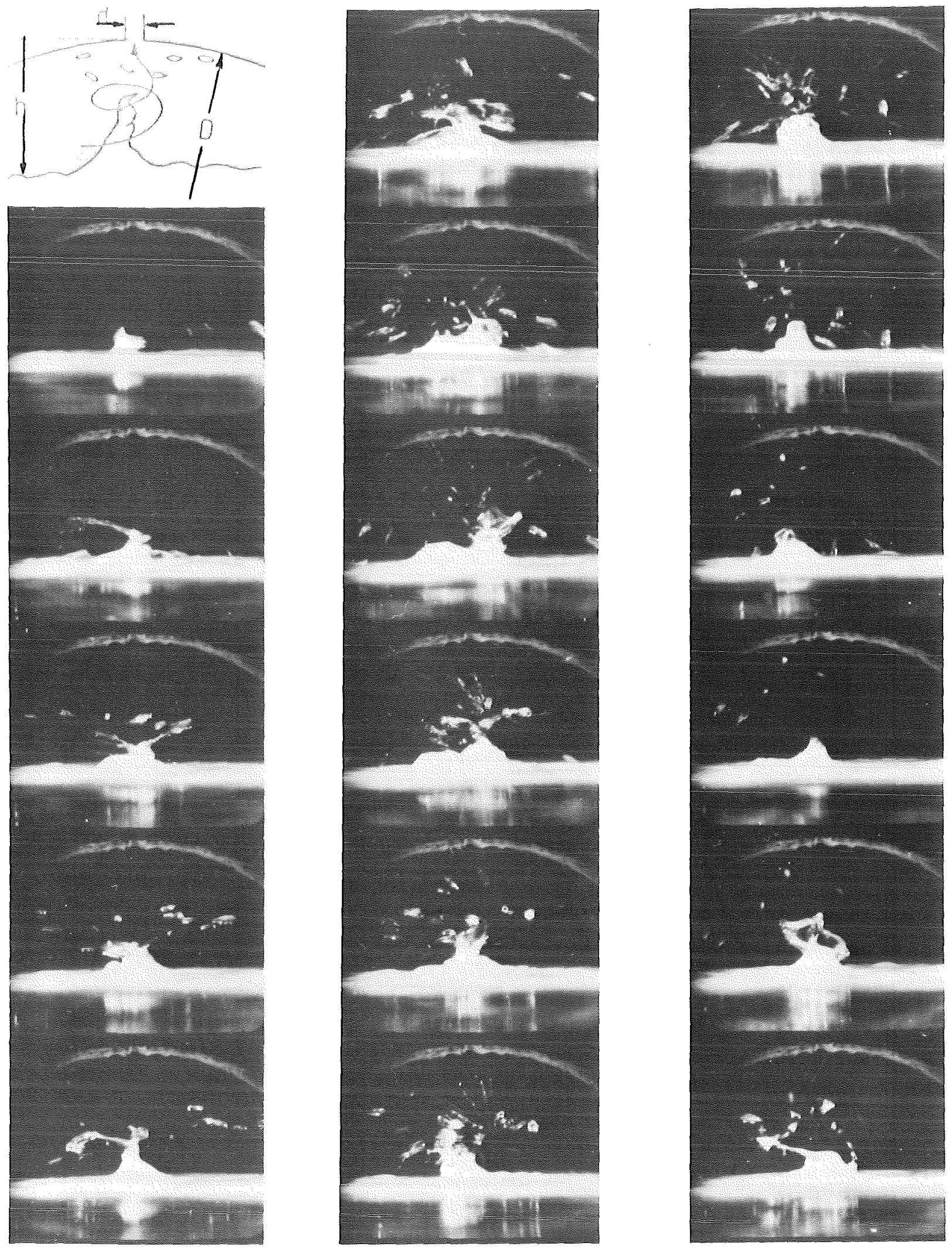

Fig. 12: Oscillatory liquid entrainment in upward branch: $\mathrm{h} / \mathrm{D}=0.3 ; \mathrm{W}_{3 \mathrm{~g}}=0.06 \mathrm{~kg} / \mathrm{s} ; \mathrm{P}_{1}=0.4 \mathrm{MPa}($ from $/ 21 /$ ). 

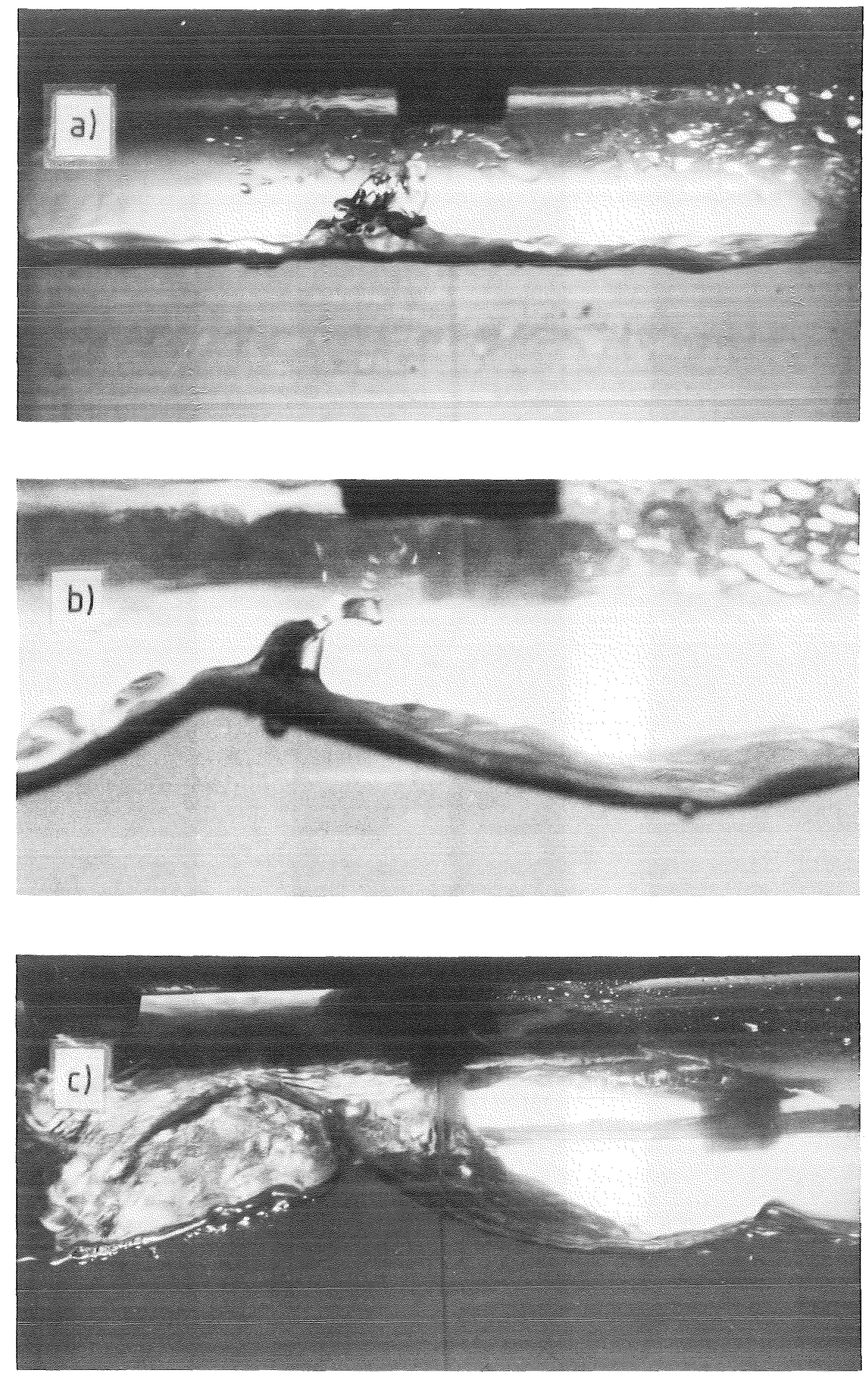

Fig. 13: Liquid entrainment in upward branch for different flow regimes in the horizontal pipe and $v_{2 g}=0$ (Erom /21/): a) stratified, b) wavy, c) slugging 


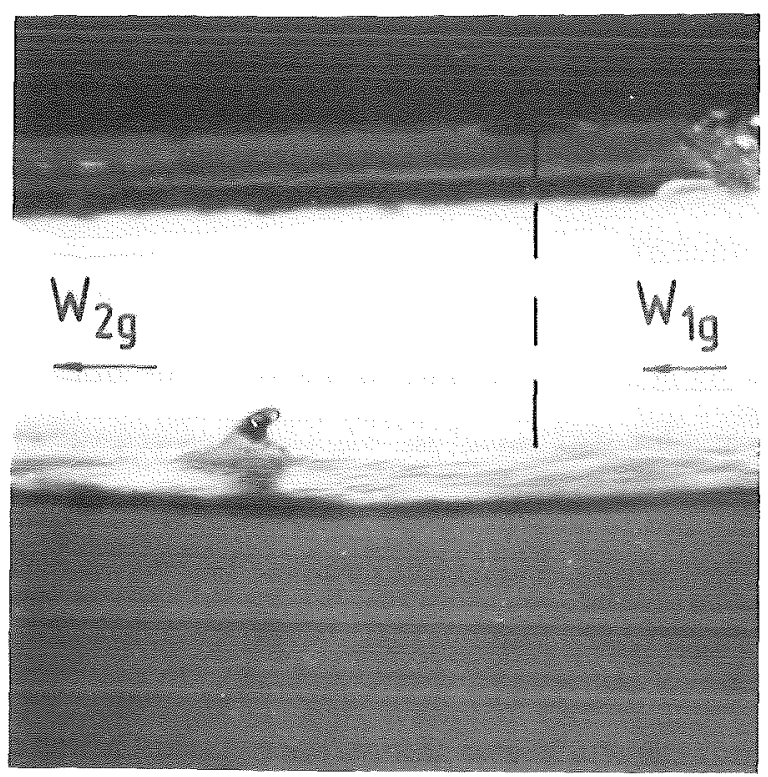

The effect of superimposed gas velocities was a slight displacement of the vortex axis and deflected interface in the direction of the outflow. This phenomenon is illustrated by the photographs in Fig. 14 corresponding to experiments with $v_{2 g} \simeq 0.4 \mathrm{~m} / \mathrm{s}$. The comparison with Fig. 13 shows an increased distance between the raised interface and the branch axis which again delays the beginning, of entrainment. Higher values of the superimposed gas velocities produce the waves

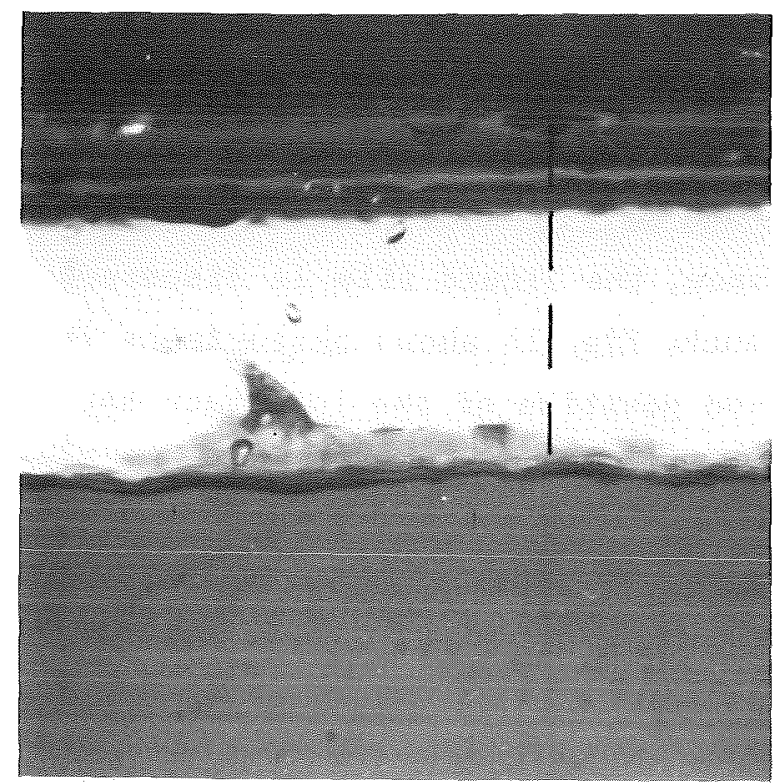
and slug flow described above and, due to this limitation in the maximum value of $v_{2 g}$, the transition from vortex to vortex free flow with increasing superimposed velocities could not be observed.

No effect of the superimposed 1iquid velocities on the position of the deflected interface was observed in the range covered by the present experiments $\left(v_{21} \leqslant 0.12 \mathrm{~m} / \mathrm{s}\right)$.

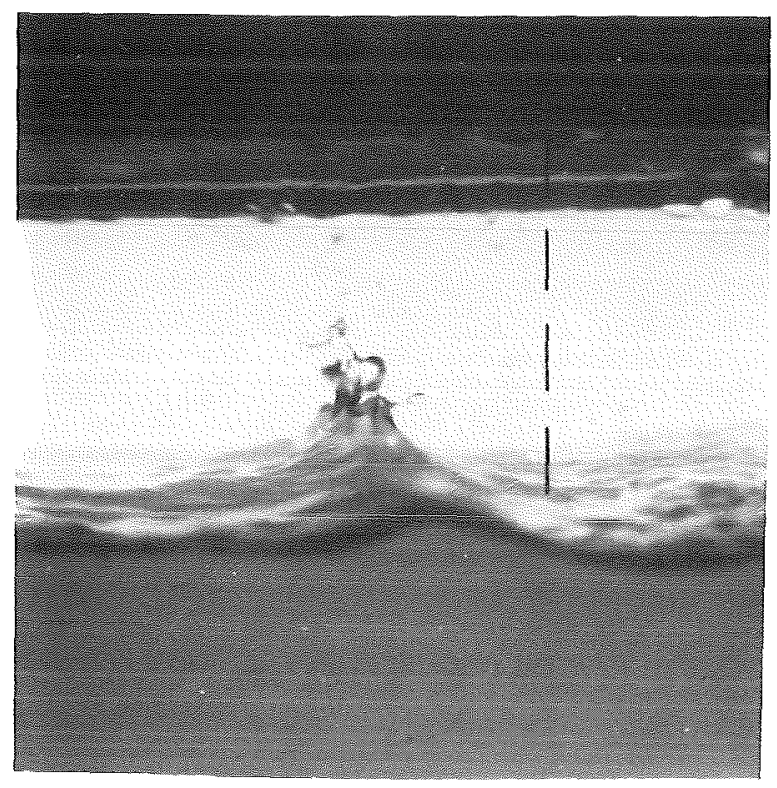

Fig. 14:

Beginning of liquid entrainment in upward branch for increasing $h_{1}$; $v_{2 g} \cong 0.4 \mathrm{~m} / \mathrm{s}$. 


\subsection{Horizontal Branch}

For this geometry the flow field configuration is clearly different from those observed in downward and upward branches.

With the interface below the branch axis again liquid entrainment can occur. The Bernoulli effect is first evidenced by the deflection of the interface in the vicinity of the pipe wall and, with further increase of the liquid level, a thin ascendent film of water, not influenced by vorticity, determines the b.l.e. With a superimposed gas velocity no appreciable displacement of this water film occurs because the friction forces dominate at the wall. Again no influence of the superimposed liquid velocities on the ascendent liquid film is observed.

The photographs in Fig. 15 show the ascendent liquid during entrainment for different liquid levels in the main pipe and $\Delta P_{1-34} \simeq 0.04 \mathrm{MPa}$. Due to the strong acceleration near the branch entrance, the liquid film is dispersed into very small droplets entering the branch. Fig. 16 shows photographs in the direction of the pipe axis, for a fixed position of the interface and increasing pressure differences. For the higher $\Delta \mathrm{P}_{1-34}$ (Fig. 16c), the dispersed flow near the entrance is clearly seen.

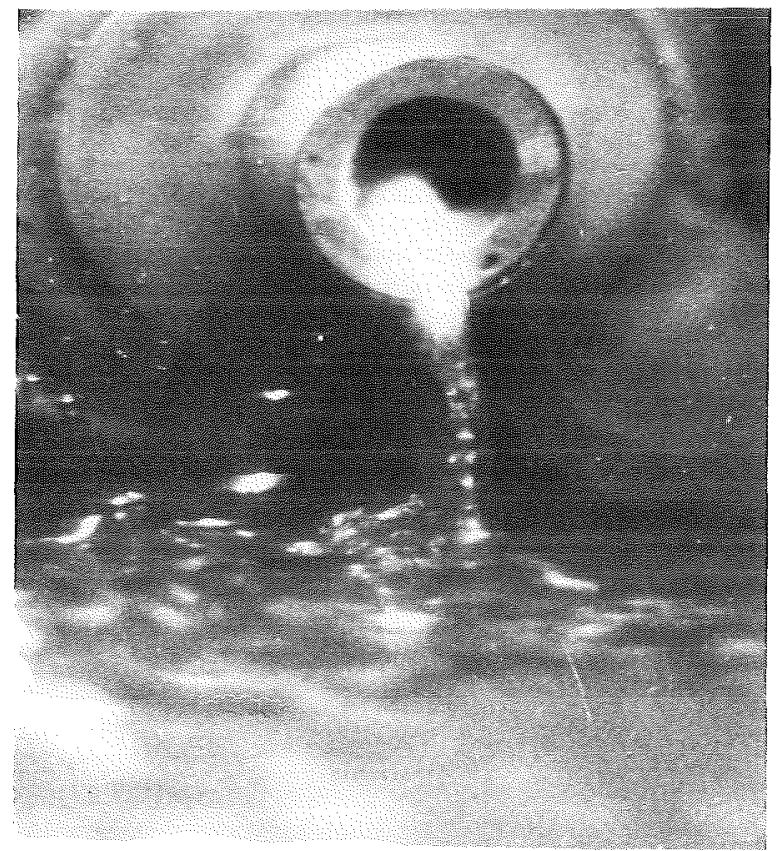

a) $\mathrm{h}_{1}=176 \mathrm{~mm}$

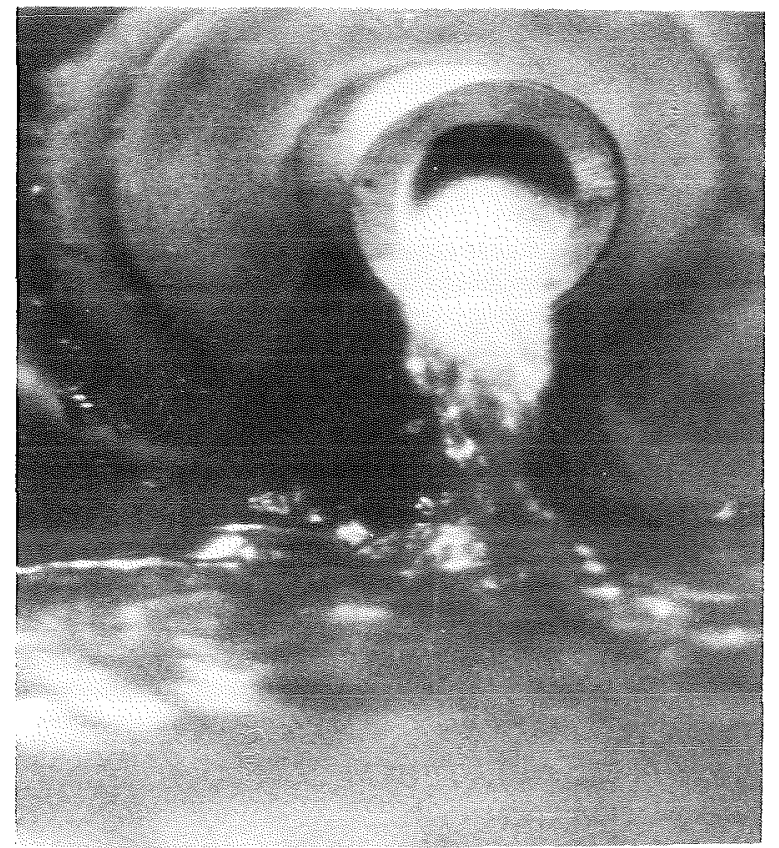

b) $\mathrm{h}_{1}=182 \mathrm{~mm}$

Fig. 15: Liquid entrainment in horizontal branch: $\Delta \mathrm{P}_{1-34} \cong 0.04 \mathrm{MPa}$. 


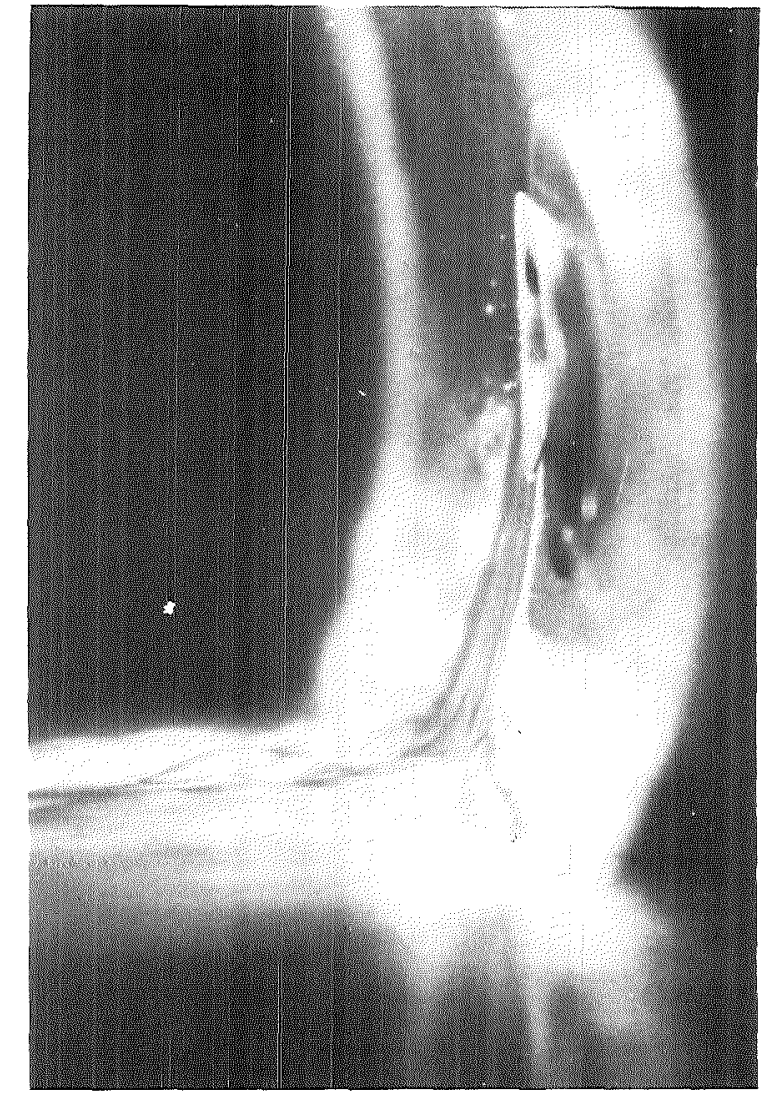

a) $\Delta \mathrm{P}_{1-34}=0.02 \mathrm{MPa}$,

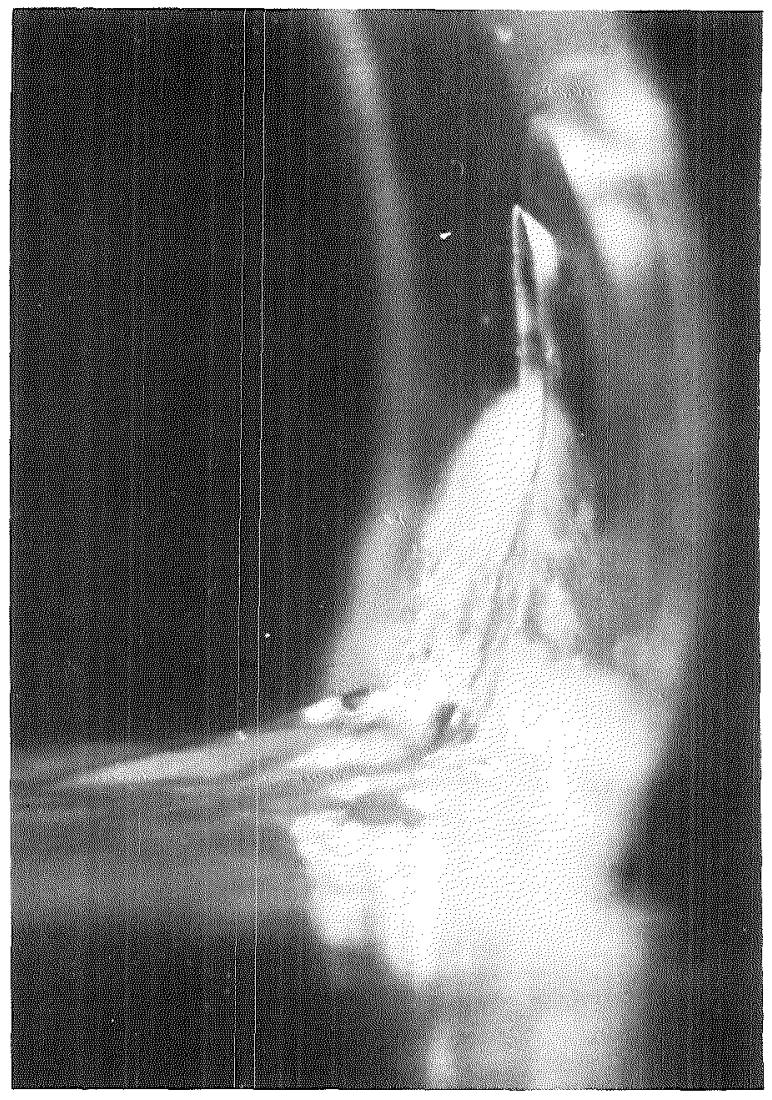

b) $\triangle P_{1-34}=0.04 \mathrm{MPa}$,

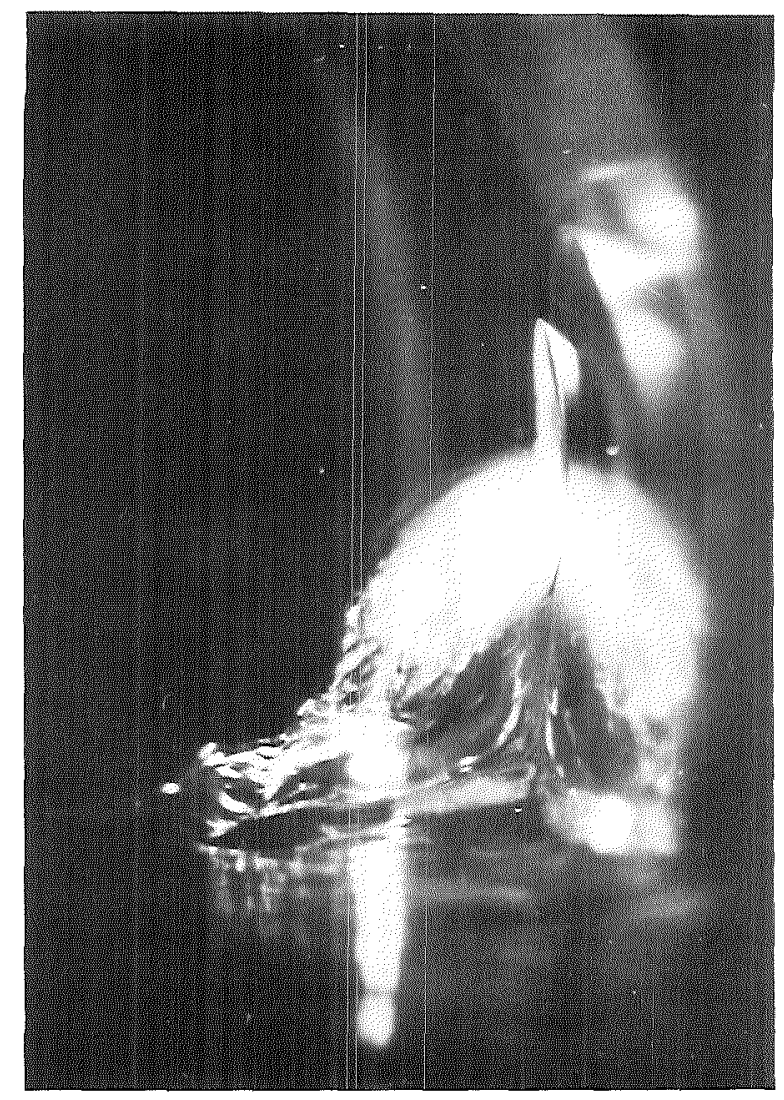

c) $\Delta \mathrm{P}_{1-34}=0.06 \mathrm{MPa}$.

Fig. 16: Liquid entrainment in horizontal branch for increasing

$$
\Delta P_{1-34}, \quad h_{1}=183 \mathrm{~mm}
$$


With the liquid level above the branch axis, again gas entrainment can occur. The continuous liquid phase exhibits a very small vorticity difficult to observe on the interface. Along the vortex axis, at a short distance from the pipe wall, thin hoses of gas reach the branch entrance and determine the b.g.e. If the interface level is further lowered or $\Delta \mathrm{P}_{1-34}$ increased, the initial hoses of gas are observed to approach rapidly the pipe wall. The wall friction prevent the vorticity from developing and after short intermittences the gas is in direct contact with the wal1. Thus, the vorticity is completely suppressed and a vortex free gas flow exist between the liquid and the pipe wall.

\subsection{Transition from Stratified to Slug Flow}

For high interface levels in the main pipe, which were of interest in the investigation involving upward branches, the transition from stratified to slug flow occurred. Specific experiments were performed to determine the conditions for this transition: With the valve for water drain closed, sufficient liquid was provided to fill the main pipe up to the desired level. Then a slowly increasing gas flow rate was adjusted by opening gradually the valve $\mathrm{V} 1 \mathrm{~g}$. The air was released to the atmosphere either through valve $\mathrm{V} 2 \mathrm{~g}$ or through the branch. The evolution of the flow field was observed visually: at low air flow rates, the water surface was horizontal and smooth, in the sense that only capillary waves or small ripples with $\lambda<5 \mathrm{~cm}$ were observed. With these disturbances the effect of the surface tension acting as a restoring force is comparable with the effect of gravity; therefore, these waves are unable to propagate and disappear almost instantaneously if the air flow is suppressed (see Lighthil1/22/). A higher air flow gave rise to uniform small waves, with $\lambda>10 \mathrm{~cm}$, for which the influence of the surface tension is negligible (compare /22/) and therefore they can be considered as pure gravity waves。

With an increasing gas flow, the action of the air moving in the same direction as the waves but at a greater velocity, is an excess of pressure on the rear slopes and a tangential drag on the crests. The wave amplitude will tend to increase to such an extent that the dissipation balances the work done by the surface forces (see e.g. Lamb /23/). Thus, the air flow was increased until waves developed whose crests reached the top of the pipe: the air flow rate causing this slugging flow, was recorded and a new experiment with a 
different initial interface level was performed.

To analyze the experimental data, the model proposed by Taitel and Dukler 124, 25/ was first considered. The authors assume that the transition takes place as a result of the Kelvin-Helmholtz instability causing waves "of finite size on the stratified film. The wavelength at which the stratified flow becomes unstable depends on gravity and surface tension, and the instability is produced by the decrease of the pressure over the waves, due to the Bernoulli effect when gas accelerates locally over the liquid. The transition from stratified to elongated bubble or slug flow patterns occurs when the lifting Bernoulli forces resulting from the decrease of the pressure over the wave, overcome the downward gravity forces.

On this basis, Taitel and Dukler deduced a relationship, valid for horizontal round pipes which allows to predict the gas phase velocity at which the transition occurs:

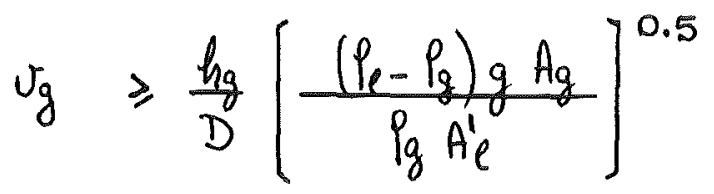

where: $A^{\prime}{ }_{1}=D\left[1-\left(2 h_{1} / D-1\right)^{2}\right]^{1 / 2}$

$v_{g} \quad=$ gas phase velocity

$h_{g} \quad=$ gap of gas above the liquid

$\mathrm{h}_{1}=$ height of the liquid from the pipe bottom

D = diameter of the pipe

$f_{1, g}=1$ iquid, gas density

$\mathrm{g} \quad=$ acceleration of gravity

$\mathrm{A}_{\mathrm{g}} \quad=$ gas-flow cross-sectional area

Eq. (1) predicts a decrease of $v_{g}$ with decreasing $h_{g}$. In the present experiments however, independent of the liquid level in the main pipe, a nearly constant value of the gas phase velocity was measured at the transition. The results are shown in Fig. 17 together with the model proposed by Taitel and Dukler. The method described in Appendix Al was used to calculate bars of error for points with extremal coordinates. 


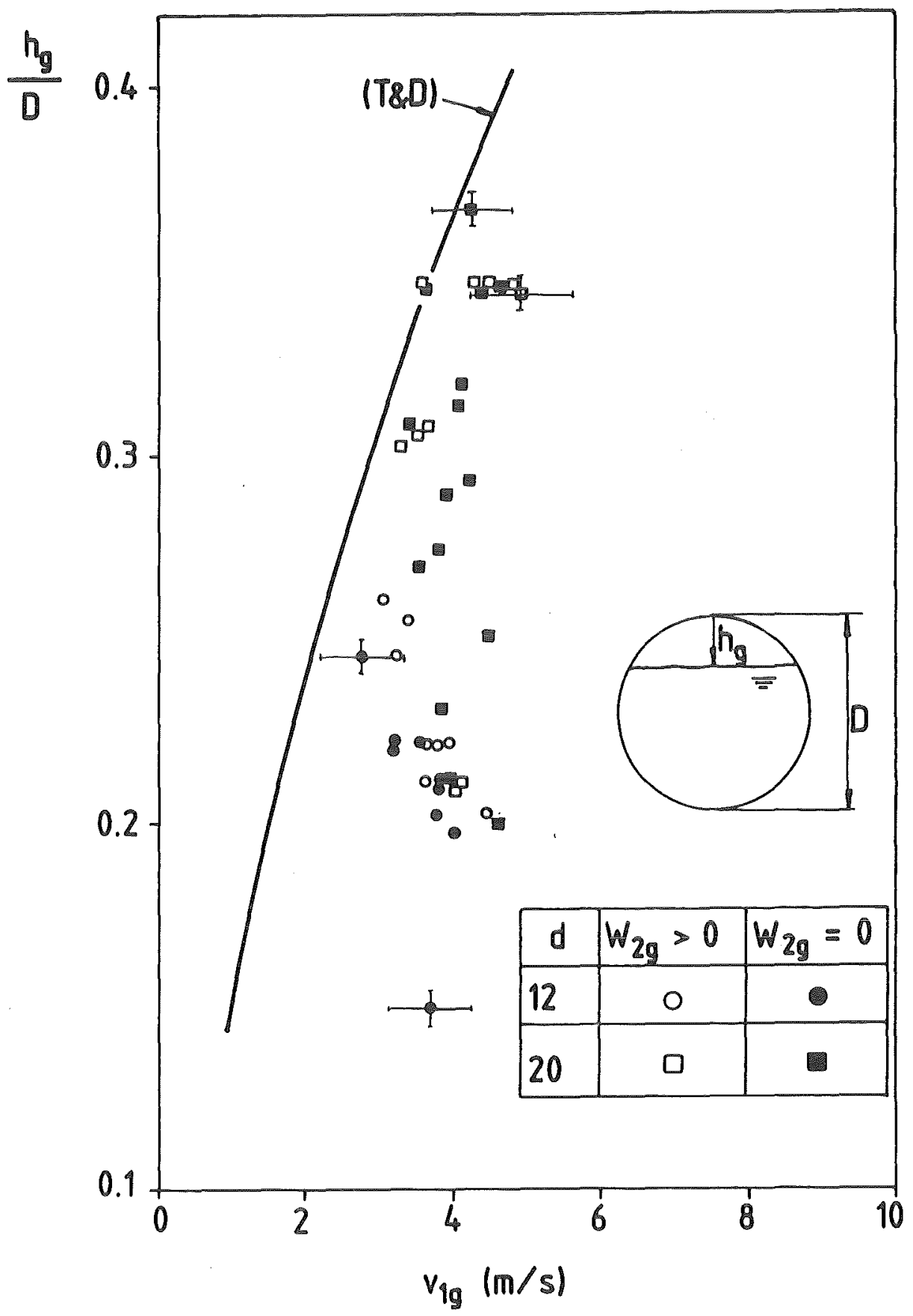

Fig. 17: Transition from stratified to slug flow. Comparison of experimental data with theoretical model from Taitel and Dukler $/ 24,25 /$.

The discrepancy between the present results and the model from Taitel and Dukler was evaluated by plotting the ratio of measured $v_{l g}$ to the value given by Eq. (1), as a function of $h_{g} / D$. The results are shown in Fig. 18a. 


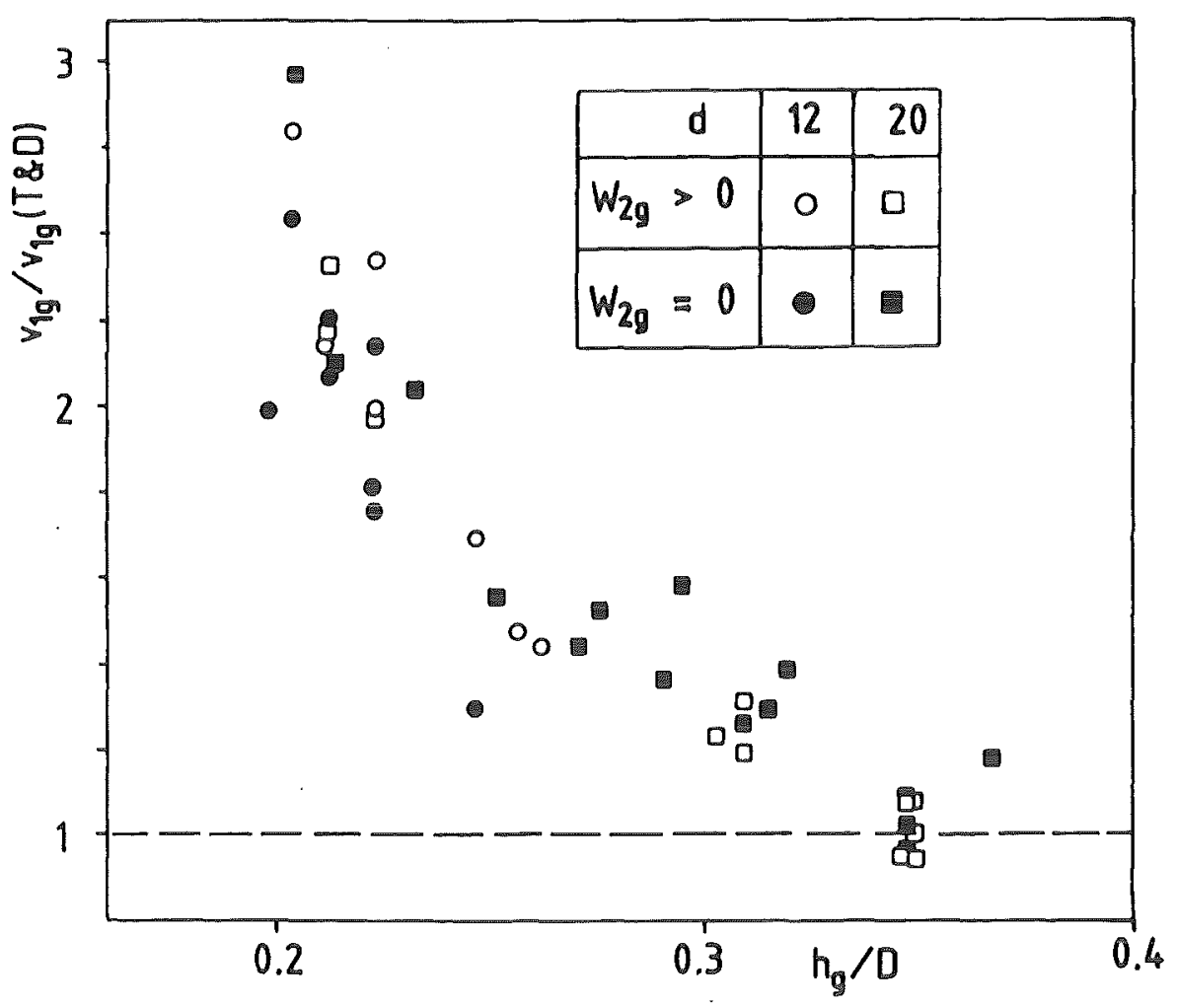

a) Ratio of measured to theoretical values from Taitel and Dukler /24, 25/

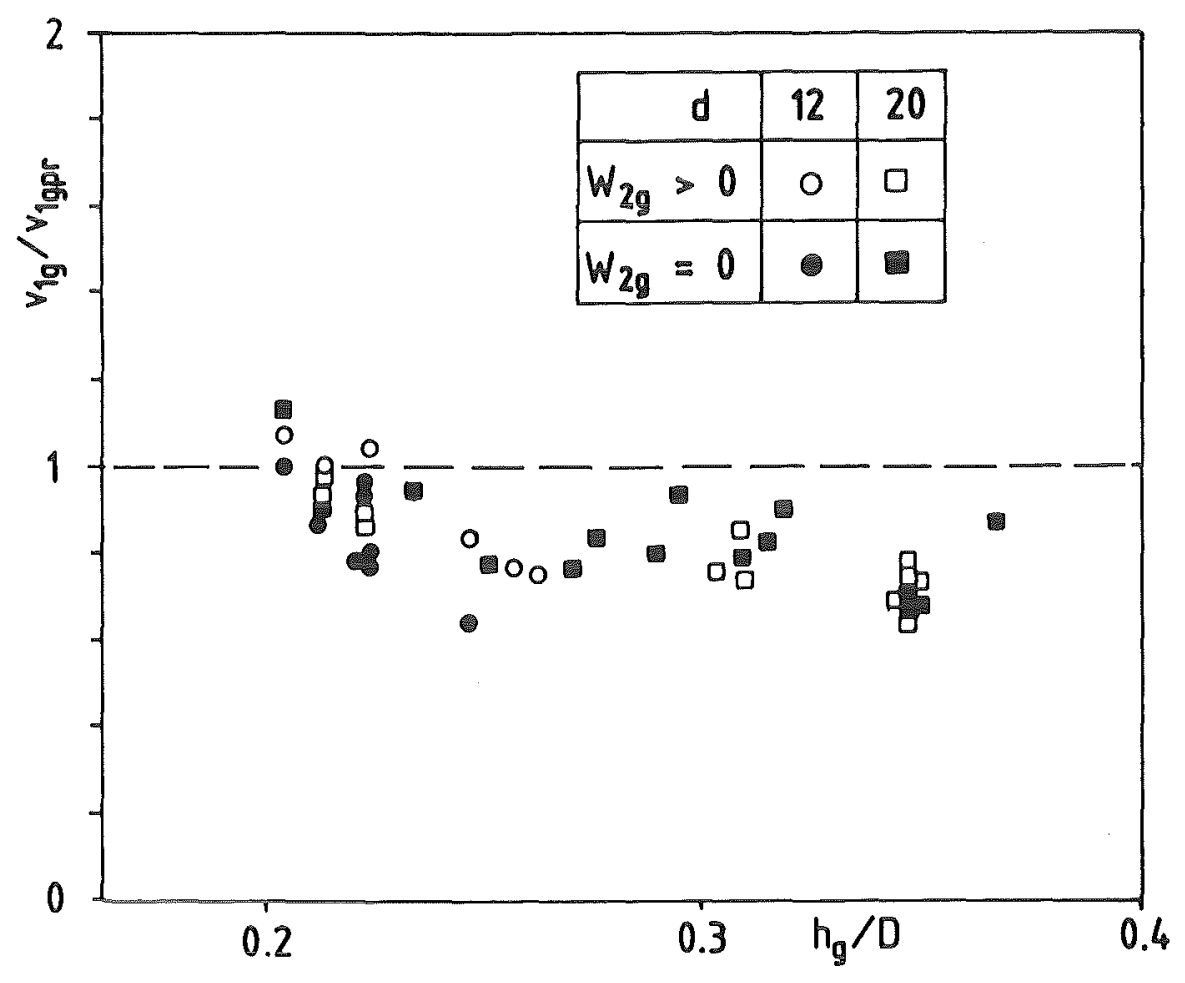

b) Ratio of measured to predicted values 
Wallis and Dobson $/ 26 /$ deduced a relationship for the onset of slugging in rectangular ducts which can be written as follows:

$$
v_{g} \geq 0.5\left[\frac{\left(\rho e-\rho_{g}\right) g \lg }{\rho_{g}}\right]^{0.5}
$$

where 0.5 is an experimental coefficient.

The authors attribute the discrepancy of this coefficient with the greater value predicted by the classical theory of the Kelvin Helmholtz instability, to the fact that it is an essentially one-dimensional theory. They argue that two-dimensional flows over large waves of finite wavelength, give rise to larger dynamic forces than those predicted by the simple theory.

If the factor $h_{g} / D$ in $E q .(1)$ is replaced by the coefficient 0.5 proposed by Wallis and Dobson, the transition is described as follows:

$$
v_{g} \geq 0.5\left[\frac{\left(\rho_{e}-P_{g}\right) g A g}{P_{g} A^{\prime} l}\right]^{0.5}
$$

which indicates that $\mathrm{v}_{\mathrm{g}}$ depends on $\mathrm{h}_{\mathrm{g}}$, only through the ratio $\mathrm{A}_{\mathrm{g}} / \mathrm{A}^{\prime}{ }_{1}$, or in other words, the transition is dominated by the gas phase velocity and not by the gap of gas.

Using Eq. (3) again the ratio of measured to predicted values of $v_{g}$ was plotted as a function of $h_{g} / D$ as shown in Fig. $18 \mathrm{~b}$.

The agreement with the model is fairly good, even if the measured values are about $20 \%$ lower than the predicted ones, which may be partly due to a different criterion employed to determine the onset of the transition. It is interesting that the experimental data were not remarkably influenced by the way the gas was leaving the test section, i.e. through valve $\mathrm{V} 2 \mathrm{~g}$ or through the branch. This indicates that the transition is not governed by the local upward acceleration of the gas in the vicinity of the branch entrance but rather by the gas moving in the direction of the waves and favoring the increase of the waves amplitude. 
5. Beginning of Gas and Liquid Entrainment

\subsection{Dimensional Analysis}

To describe the beginning of gas or liquid entrainment, a general case is illustrated in Fig. 19 where again the labels 1, 2, 3 are used to distinguish between inflow, outflow and branch-flow, respectively.

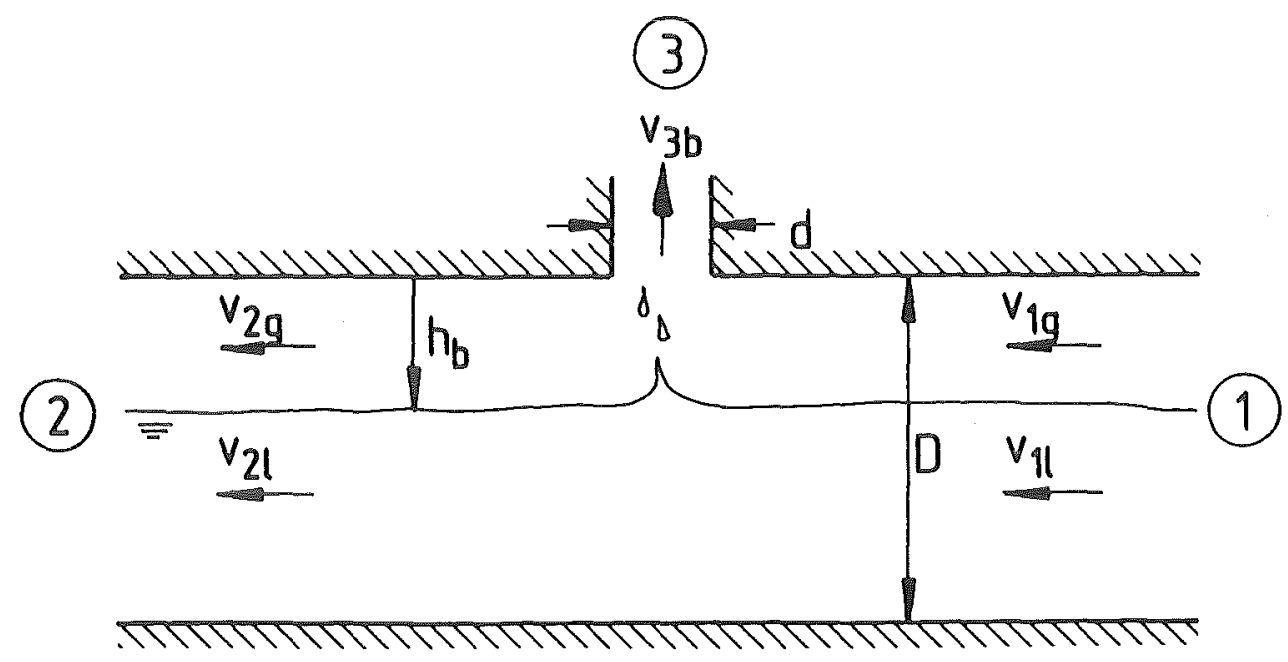

Fig. 19: General variables to determine the beginning of entrainment.

If the effects of viscosity and surface tension are neglected, the significant variables for the determination of the beginning of entrainment (b.e.) can be classified as follows:

Geometrical parameters:

$\mathrm{h}_{\mathrm{b}}=$ distance from the branch entrance to the interface at the beginning of entrainment

$\mathrm{d}=$ diameter of the branch

D $=$ diameter of the main pipe

Kinematic quantities:

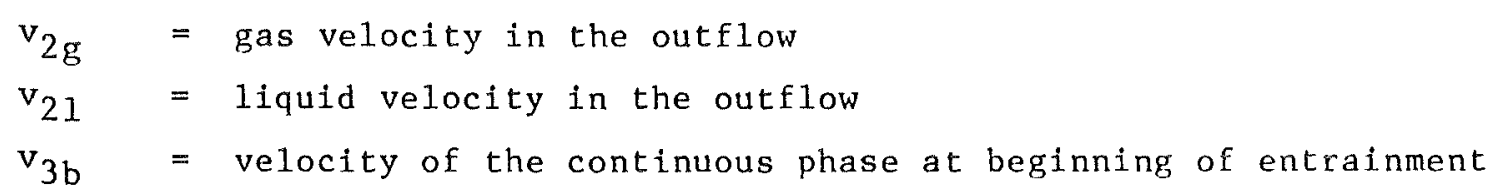
Fluids properties:

$\mathrm{P}_{\mathrm{b}}=$ density of the continuous phase at beginning of entrainment

$P_{1}-\rho_{g}=$ difference between liquid and gas densities

External forces:

g $\quad=$ acceleration of gravity. 
By combination of these variables, the following dimensionless groups are chosen to describe the beginning of entrainment:

$$
\frac{h_{6}}{d}, \frac{\rho_{6} v_{36}^{2}}{g d\left(\rho_{l}-\rho_{g}\right)}, \frac{\rho_{g} v_{2 g}^{2}}{\rho_{b} v_{36}{ }^{2}}, \frac{\rho_{l} v_{2 l}^{2}}{\rho_{6} v_{36}{ }^{2}}, \frac{d}{D}
$$

The first group is the most important geometrical parameter since it represents the influence of the break size on the beginning of entrainment; the second constitutes the ratio of inertial to gravitational forces in the branch flow (Froude number $\mathrm{Fr}$ ); the following two terms are ratios between momentum-fluxes and constitute a relative magnitude of the superimposed velocities; the last group is a geometrical parameter indicating the relative size of the break.

The magnitude of the various dimensionless numbers for the present experiments with different branch flow configurations, are summarized in Table 2 which also indicates whether a vortex or a vortex-free flow was observed in the continuous phase.

\begin{tabular}{|c|c|c|c|c|c|}
\hline \multicolumn{1}{|c|}{} & vortex flow & vortex free & vortex flow & vortex flow & vortex flow \\
\hline$\frac{h_{b}}{d}$ & $2.8-12$ & $1.5-5.5$ & $2.5-7$ & $0.4-3.8$ & $0.5-3.2$ \\
\hline$\frac{v_{3 b}^{2} \rho_{b}}{g d\left(\rho_{1}-\rho_{g}\right)}$ & $1-5 \times 10^{3}$ & $2 \times 10^{2}-5 \times 10^{3}$ & $4-50$ & $2-1.8 \times 10^{3}$ & $4-4.8 \times 10^{3}$ \\
\hline$\frac{\rho_{g} v_{2 g}^{2}}{\rho_{b} v_{3 b}^{2}}$ & $0-10^{-5}$ & $0-10^{-5}$ & $0-1.5 \times 10^{-4}$ & $0-3 \times 10^{-3}$ & $0-10^{-3}$ \\
\hline$\frac{\rho_{1} v_{21}^{2}}{\rho_{b} v_{3 b}^{2}}$ & $0-10^{-6}$ & $0 ; 10^{-6}-10^{-4}$ & $0-1.6 \times 10^{-4}$ & $0-1.7 \times 10^{-3}$ & $0-3 \times 10^{-3}$ \\
\hline$\frac{d}{D}$ & $<10^{-1}$ & $<10^{-1}$ & $<10^{-1}$ & $<10^{-1}$ & $<10^{-1}$ \\
\hline
\end{tabular}

Table 2: Range of values adopted by the dimensionless groups chosen to describe the beginning of entrainment. 
For downward branches the data corresponding to b.g.e. with superimposed liquid velocities were taken from previous results $/ 17,18 /$. In those experiments (see section 3.2) a small gas flow rate was used to keep the system pressure constant; this is indicated by the range $0-10^{-5}$ assigned to the group $\rho_{g} v_{2 g}{ }^{2} / \rho_{b} v_{3 b}{ }^{2}$.

In agreement with the description in Section 4.1 a vortex free flow in downward branches occurred only with $\rho_{1} v^{2}{ }_{21} / \rho_{b} v^{2}{ }_{3 b}$ equal to zero or greater than $10^{-6}$.

Table 2 shows that, in the range of the experiments, the momentum flux in the outflow is at least three orders of magnitude lower than the momentum flux in the branch. Therefore, no significant influence is expected of the superimposed velocities on the boe. and the groups containing $v_{2 g}$ or $v_{21}$ may be discarded. The ratio $d / D$ was always smaller than 0.1 . This means that for the investigated branches a geometrical similitude can be assumed for the various flow fields and the b.e. can be considered to be independent of $d / D$. Therefore only the correlation between $h_{b} / d$ and the Froude number is investigated below. 1)

\subsection{A General Correlation for the Beginning of Entrainment}

On the basis of the dimensional analysis made above the following correlation is proposed to describe the b.e. in branches with arbitrary orientations:

$$
F_{r}^{a}=k^{*} \frac{h_{b}}{d} \quad \text { or } \quad\left[\frac{v_{3 b}^{2} P_{b}}{g d\left(P_{l}-P_{g}\right)}\right]^{a}=k^{*} \frac{h_{b}}{d}
$$

where $\mathrm{a}$ and $\mathrm{K}^{*}$ are constants to be determined from the experiments.

With $v_{3 b}=v_{31}$ and $P_{b}=P_{1}$, Eq. (4) is identical to the correlation used in $117,18 /$ to describe the b.g.e. in downward branches. The authors fitted their experimental data with $a=0.2$ and proposed to substitute $v_{3 b}$ in the Froude number by the form $\omega_{3 b} / \rho_{b} \pi d^{2} / 4$ to get the following correlation which does not depend explicitly of $d$ :

$$
\frac{l_{6}\left[g p_{b}\left(P_{l}-P_{g}\right)\right]^{0.2}}{W_{3 b}^{0.4}}=k
$$

From this result the authors concluded that for $h_{b} / d \gg 1$ the flow field

1) This conclusion is based on a Taylor expansion of the implicit function of all relevant dimensionless groups. 
generated by the branch is equivalent to that due to a punctual sink at the branch entrance, i.e., it is dominated by the branch mass flow rate and not by the branch diameter.

This equivalence with a punctual sink was also used by Craya /12/ who described analytically the b.1.e. in a side orifice at a distance $h$ above the interface between two liquids of densities $P$ and $(\rho+\Delta P$ ) with a correlation like (5). From considerations of equilibrium at the interface (see Appendix A2), Craya obtained:

$$
\frac{\Delta p}{p_{6}} g \frac{h_{6}^{5}}{q^{2}}=\text { constant }
$$

where $q$ represents the volume flow through a three-dimensional punctual sink at the orifice outlet Eq. (6) was experimentally confirmed by Gariel /13/. At b.1.e.: $q=W_{3 b} / P_{b}$, therefore by replacement of the lighter liquid by gas: $\Delta P=\left(P_{1}-P_{g}\right)$, the correlation from Craya becomes:

$$
\frac{h_{b}\left[g \rho_{b}\left(P_{e}-\rho_{g}\right)\right]^{0.2}}{w_{36}^{0.4}}=K
$$

which coincides with Eq. (5).

The validity of Eq. (5) to describe the b.1.e. in upward branches is discussed below.

\subsection{Theoretical Considerations for the Beginning of Liquid Entrainment in Upward Branches}

\subsubsection{Flow Field Generated by the Break}

To determine the conditions for the b.1.e. in upward branches the flow field generated by the break was investigated by using the potential flow theory.

For stratified flow without superimposed velocities in the main pipe, the flow of the continuous phase can be described as the superposition of

- a line vortex with the axis along the branch, and

- a punctual sink at the branch entrance, bounded by a rigid wall.

The contribution of a line vortex to the velocity potential $\emptyset$ at a distance $r$ 
from the vortex axis (the branch axis) is proportional to $1 / r$, which gives a velocity, $\overrightarrow{\mathrm{V}}=-\nabla \emptyset$, proportional to $1 / \mathrm{r}^{2}$ describing circular paths around the branch axis (compare e.ge Li and Lam /27/).

This velocity does not modify the pessure drop in the direction of the branch axis and, therefore, with regard to the b.1.e., without superimposed velocities, only the flow field generated by the punctual sink at the branch inlet must be considered.

Figure 20 represents a punctual sink coinciding with the origin of cartesian coordinates $(x, y, z)$ at the branch inlet. Some streamlines are drawn to represent qualitatively the flow field corresponding to a sink bounded by a rigid plane at $z=0$ and a wall represented by the ideally horizontal interface at $z=-h$.

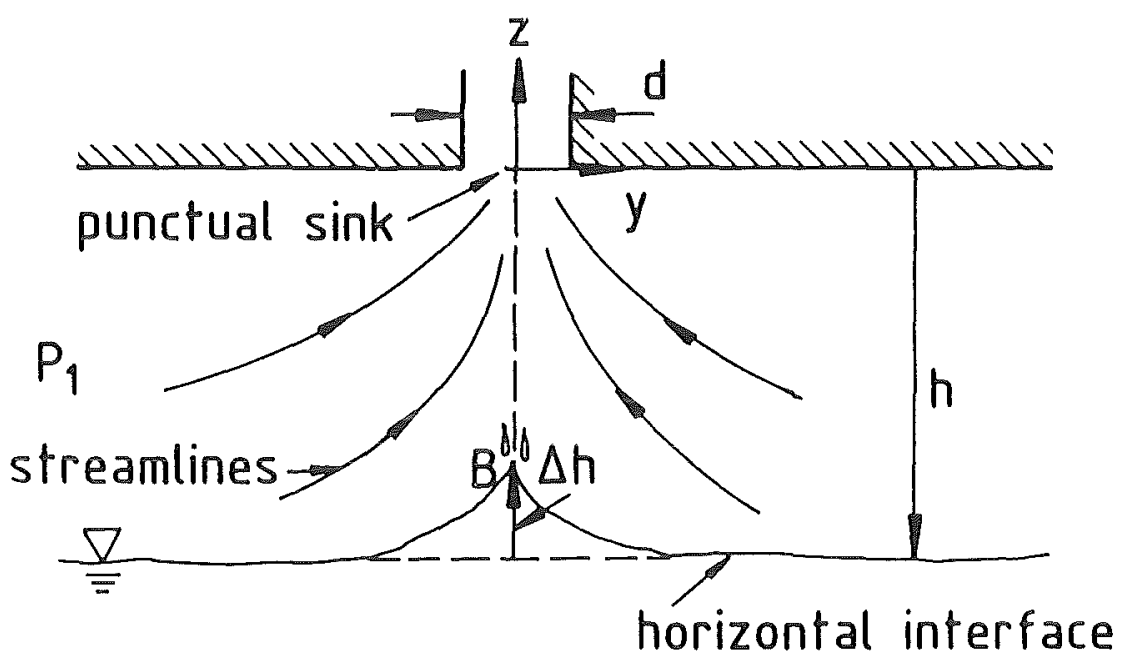

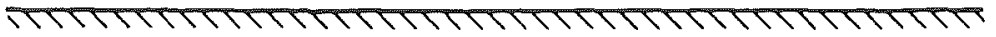

Fig. 20: Flow field generated by a punctual sink at the branch entrance.

The gas accelerated towards the sink produces a pressure drop above the liquid (Bernoulli effect) and consequently the interface is locally raised to satisfy the hydrostatic condition: Pressure and density are constant along a horizontal surface of the fluid, thus:

$$
P_{B}+g \rho_{\rho} \Delta h=P_{1}
$$

where $P_{B}$ is the local static pressure at the top $B$ of the deflected interface 
and $h$ is the corresponding height of liquid above the horizontal interface (see Fig. 20). Fluctuations of the flow field produce droplets detached from the top of the deflected interface as observed in the experiments (see Section 4.2). The b.1.e. occurs when the upward force produced by the pressure drop overcomes the gravitational force acting on the droplets. The upward force is a function of the $z$-component of the gas velocity which increases when the gas approaches the z-axis; as indicated by the corresponding increasing slope of the streamlines. Therefore, the droplets above the deflected interface and in the vicinity of the $z$-axis, will be the first to reach the branch entrance. In this region the streamlines can be assumed to be entirely radial, i.e. the influence of the interface on the velocity field, can be disregarded.

\subsubsection{Determination of the Beginning of Entrainment}

Figure 21 represents a punctual sink $S$ at the origin of the spherical coordinates $(R, \theta, \varphi)$, bounded by a rigid plane at $z=0$.
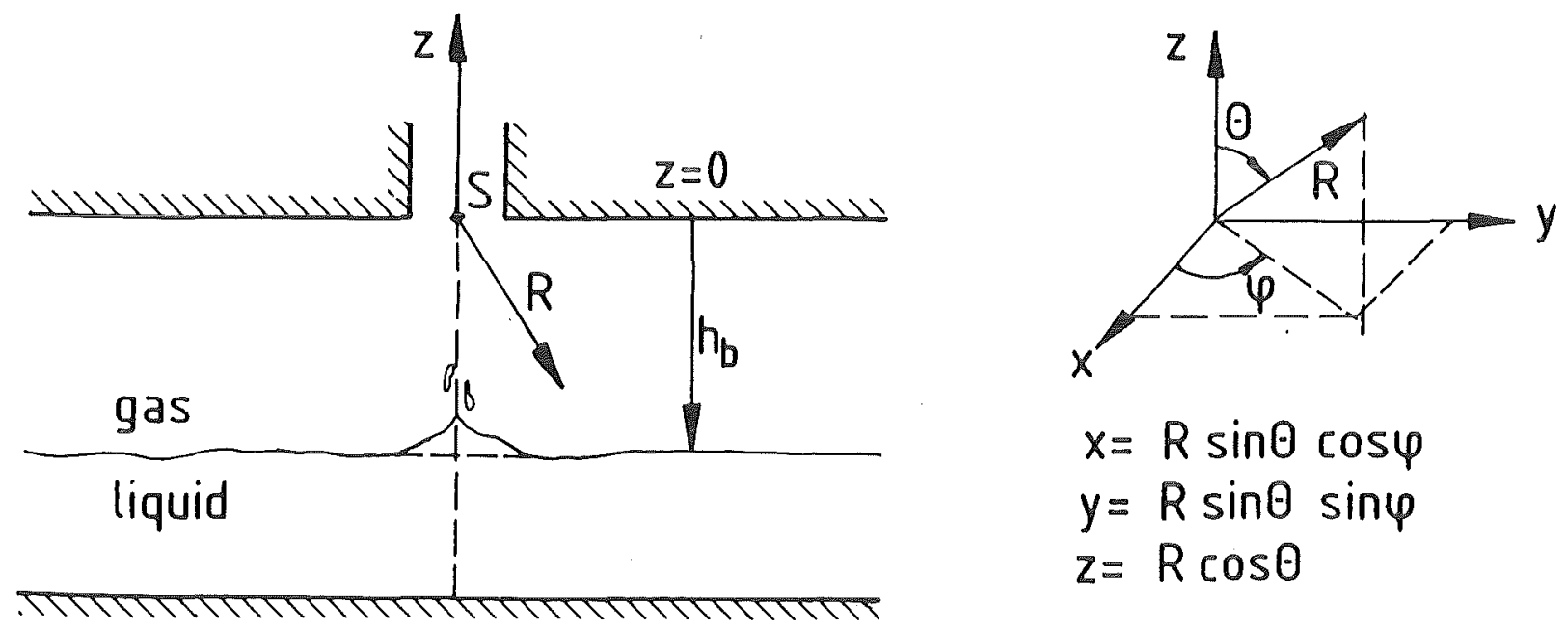

$$
\begin{aligned}
& x=R \sin \theta \cos \varphi \\
& y=R \sin \theta \sin \varphi \\
& z=R \cos \theta
\end{aligned}
$$

Fig. 21: Definition of the coordinates used to describe the beginning of liquid entrainment in upward branches.

The corresponding velocity potential is (see e.g. /27/):

$$
\phi=-2 q / 4 \pi R
$$

where $q=$ volume flow through the sink

$R=$ distance from the sink. 
Thus, the velocity field generated by the sink, $\vec{V}=-\nabla \phi$, is entirely radial:

$$
\mu=-\frac{\partial \phi}{\partial R}=-\frac{2 q}{4 \pi R^{2}}
$$

which in terms of the variables used to describe the branch flow, becomes:

$$
u=-\frac{W_{3 g}}{2 \pi \lg R^{2}}
$$

The condition for the b.1.e., can be written in terms of the forces acting on a droplet detached from the interface as follows:

$$
F_{p}+F_{g}=0
$$

where $F_{p}$ is the force due to the pressure drop along the $z$-axis and $F_{g}$ is the gravitational force.

Along the streamline represented by the $z$-axis the Bernoulli equation takes the form:

$$
\frac{1}{2} \rho g u^{2}+P+\rho g g z=\text { constant }
$$

In this particular streamline the coordinate $z=R \cos \theta$ takes the value $z=R \cos \pi=-R$; therefore, the pressure distribution given by the last equation is:

$$
P=-\frac{1}{2} \lg u^{2}+\lg g R+\text { constant }
$$

By differentiation, assuming $\rho_{g}$ constant in the test section, it results:

$$
\frac{d P}{d R}=-P_{g} \mu \frac{d u}{d R}+\lg g
$$

and with the value of $u$ given by Eq. (11)

$$
\frac{d P}{d R}=\frac{\omega_{3 g}}{2 \pi^{2} \rho_{g} R^{5}}+\rho_{g} g
$$

This is the upward force per unit volume due to the Bernoulli effect, acting on a liquid drop detached from the interface at a distance $R$ on the $z$-axis. At b.l.e. the distance to a drop just above the deflected interface is $R=R_{b}$ 
and the last equation takes the form:

$$
F_{p}=\frac{w_{3 g}^{2}}{2 \pi^{2} \rho_{g} R_{6}^{5}}+p_{g} g
$$

The gravitational force per unit volume of liquid is given by:

$$
\mathrm{F}_{\mathrm{g}}=-\rho_{1} \mathrm{~g}
$$

With Eqs. (17) and (18) into Eq. (12) and after rearranging, the condition for the b.l.e. is:

$$
\frac{R_{b}[g \rho g(\rho e-\rho g)]^{0.2}}{\omega_{3 g}^{0.4}}=\left(\frac{1}{2 \pi^{2}}\right)^{0.2}
$$

In experiments with upward branches, the height $\Delta$ h of the cone shaped interface at b.1.e. was observed to be about $20 \%$ of the distance $h_{b}$ measured with a horizontal interface. Thus, with $R_{b} \simeq 0.8 h_{b}$, the b.l.e. in upward branches is described by:

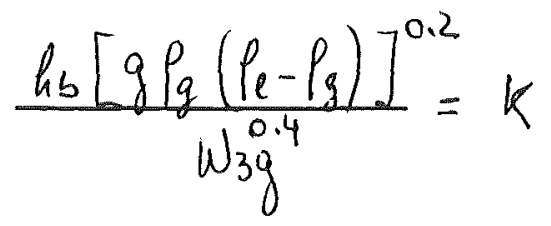

where $K=1.25\left(1 / 2 \pi^{2}\right)^{0.2} \simeq 0.688$

With $\rho_{g}$ and $W_{3 g}$ replaced by $\rho_{b}$ and $W_{3 b}$ respectively, this correlation is identical to Eq. (5) which consequently can be used to describe the beginning of entrainment in downward, horizontal and upward branches. The results in this section also confirm the hypothesis made in /17/ of equivalence between break and sink flow.

\subsubsection{Influence of Superimposed Velocities}

The shifting of the gas vortex entering upward branches, when a superimposed gas flow is applied, was described in section 4.2 and is schematically illustrated in Fig. 22 .

If the vortex is assumed to rotate along the axis CS the observations made in Section 5.3.1 that the vortex does not contribute to the Bernoulli effect de- 

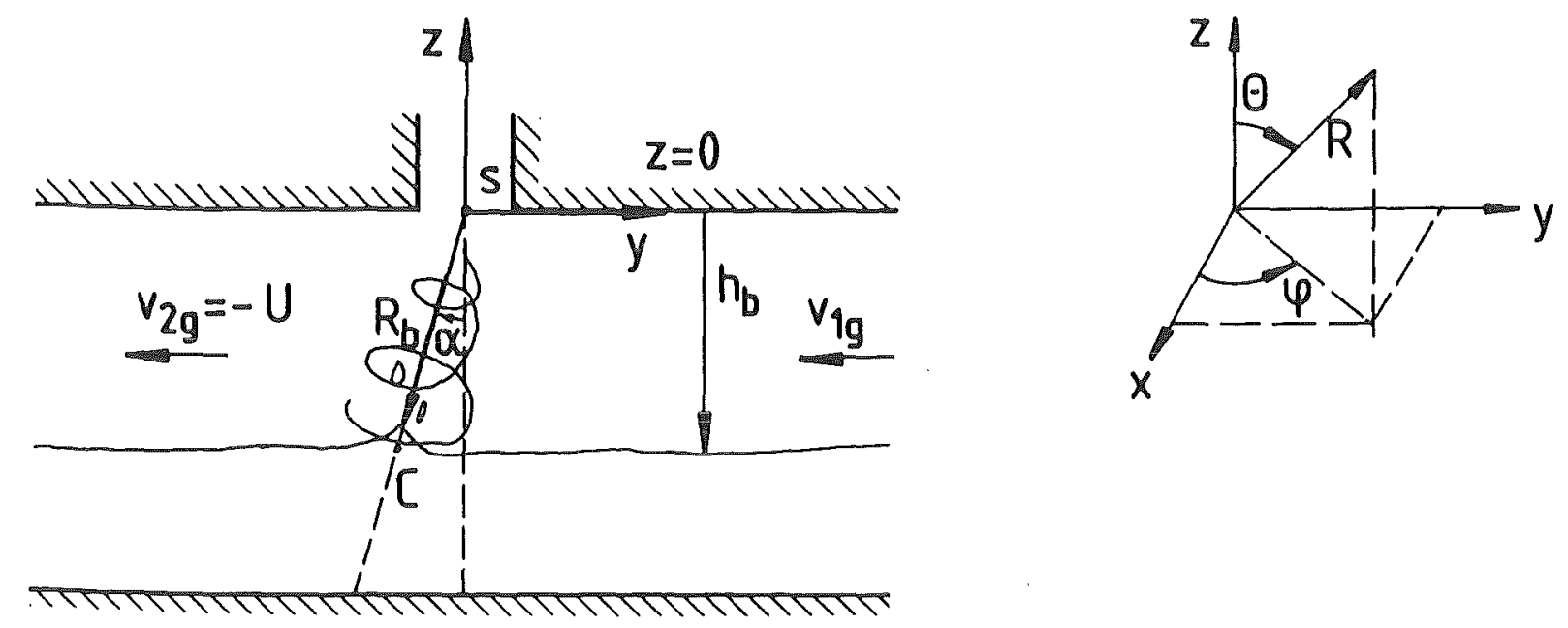

Fig. 22: Illustration of the gas vortex displacement produced by a superimposed gas flow.

termining the b.1.e., is again valid and only the uniform flow with velocity $-U$, along the $y$-axis, and the sink $S$ at the intake, must be considered. The corresponding velocity potential in spherical coordinates is:

$$
\phi=-\frac{2 q}{4 \pi R}-U R \sin \theta \sin \varphi
$$

or, with $q=w_{3 g} / \rho_{g}$ and $\varphi=-\frac{\pi}{2}$

$$
\phi=-\frac{w_{3 g}}{4 \pi \rho_{g} R}+U R \sin \theta
$$

Along the stream lines, the flow velocity $\vec{V}=-\nabla \emptyset$ has now two components but only the radial component $u=-\frac{\partial \phi}{\partial R}$ along the line CS is of interest; with $\theta=-\pi+\alpha$ it takes the value

$$
\mu=-\frac{\omega_{3 g}}{2 \pi \rho g R^{2}}+U \sin \alpha
$$

Since the velocities toward the sink are considered to be negative, Eq. (23) shows a decrease in the radial velocity proportional to $\sin \alpha$. The corresponding decrease in the Bernoulli effect is obtained by substitution of Eq. (23) and $z=-R \cos \alpha$ into Eq. (13), which, with the procedure used in Section 5.3.2, leads to the following pressure force per unit volume in the radial direction at b.1.e.: 


$$
F_{p}=\frac{\omega_{3 g}}{2 \pi^{2} \rho_{g} R_{b}^{5}}+\rho_{g} g \cos \alpha
$$

The radial component of the gravitational force per unit volume of liquid at $C$ is :

$$
F_{g}=-\rho_{l} g \cos \alpha
$$

With the two last expressions the condition for b.1.e. given by Eq. (12) becomes:

$$
\frac{h_{6}\left[g \rho_{g}\left(\rho_{e}-\rho_{g}\right)\right]^{0.2}}{\omega_{3 g}^{0.4}}=\left(\frac{1}{2 \pi^{2} \cos \alpha}\right)^{0.2}
$$

and with $\mathrm{R}_{\mathrm{b}} \simeq 0.8 \mathrm{~h}_{\mathrm{b}} / \cos \alpha$ (see Fig. 22) the final correlation is:

$$
\frac{h_{6}\left[g P_{g}\left(P_{e}-\rho_{g}\right)\right]^{0.2}}{w_{3 g}^{0.4}}=K
$$

where $K=1.25\left(1 / 2 \pi^{2}\right)^{0.2}(\cos \alpha)^{0.8} \cong 0.688(\cos \alpha)^{0.8}$.

In agreement with the smaller $h_{b}$ measured with superimposed gas flows, this result shows a diminution of the constant $\mathrm{K}$ with respect to the value for $\mathrm{v}_{2 \mathrm{~g}}$ $=0$ (Eq. (20)). This diminution is proportional to $(\cos \alpha)^{0.8}$ and, therefore, a higher reduction of $\mathrm{K}$ is expected with higher superimposed gas flow (higher $\alpha)$, as long as a stratified flow prevails. According to the description of the flow phenomena in Sec. 4.2 , no change in $K$ is expected with a superimposed liquid flow $(\alpha=0)$.

\subsection{Results}

For the three geometries under investigation $h$ represents the distance from the branch entrance (branch axis) to the interface and symbol $h_{b}$ is used to represent the distance $h$ at beginning of entrainment. For the different geometries the value of $K$ given by $E q$. (5) was plotted as a function of $h_{b} / d$.

The results shown in Figs. 23-25 correspond to b.e. without superimposed velocities. The data for b.g.e. in downward branches, Fig. 23, show a good agreement with previous results from $/ 17,18 \%$. Data for b.1.e. in upward branches (from /21/) are presented in Fig. 24 and data for b.1.e. and b.g.e. in horizontal branches (from /28/) are shown in Fig. 25. For the various 


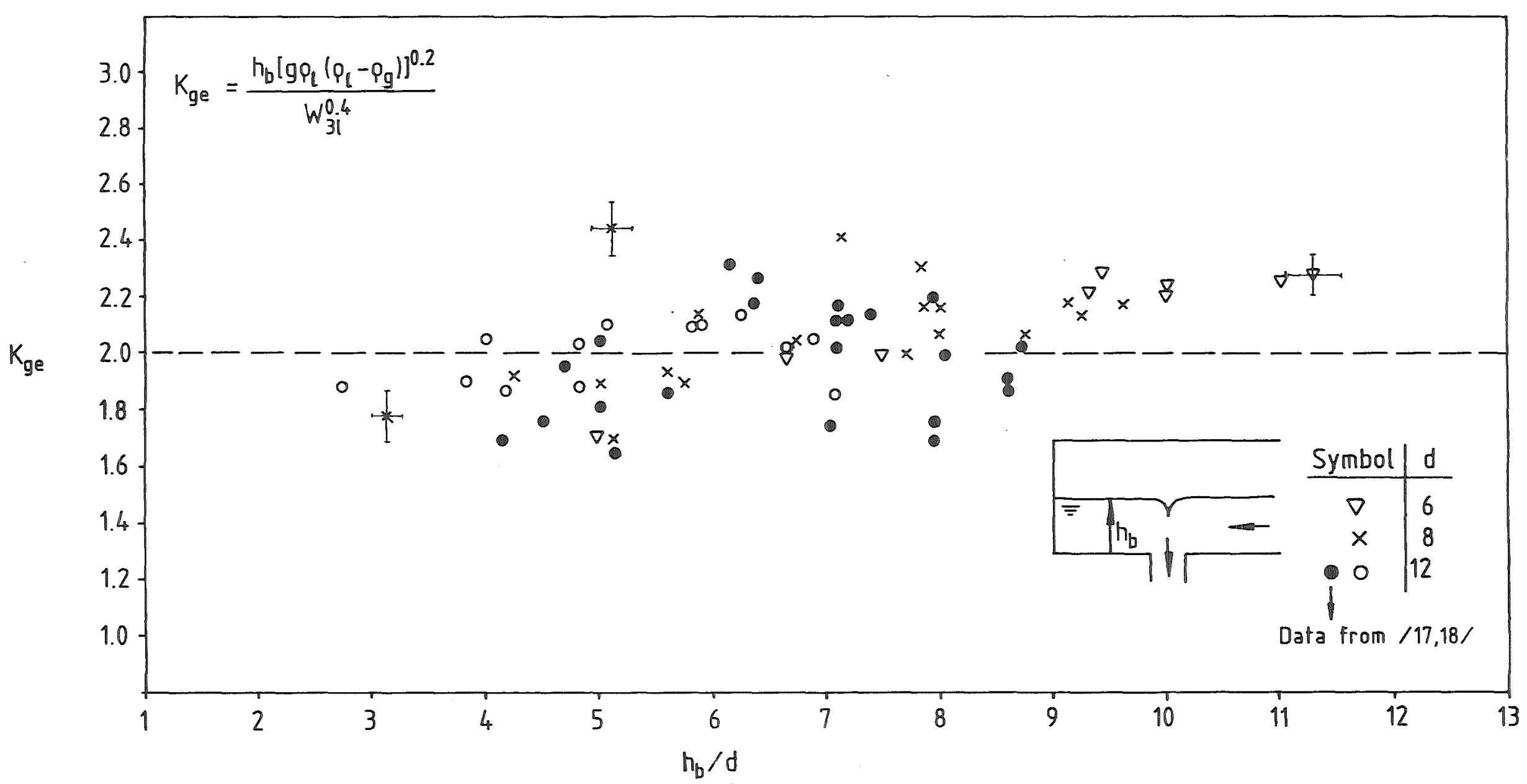

Fig. 23: Beginning of gas entrainment in downward branches. 


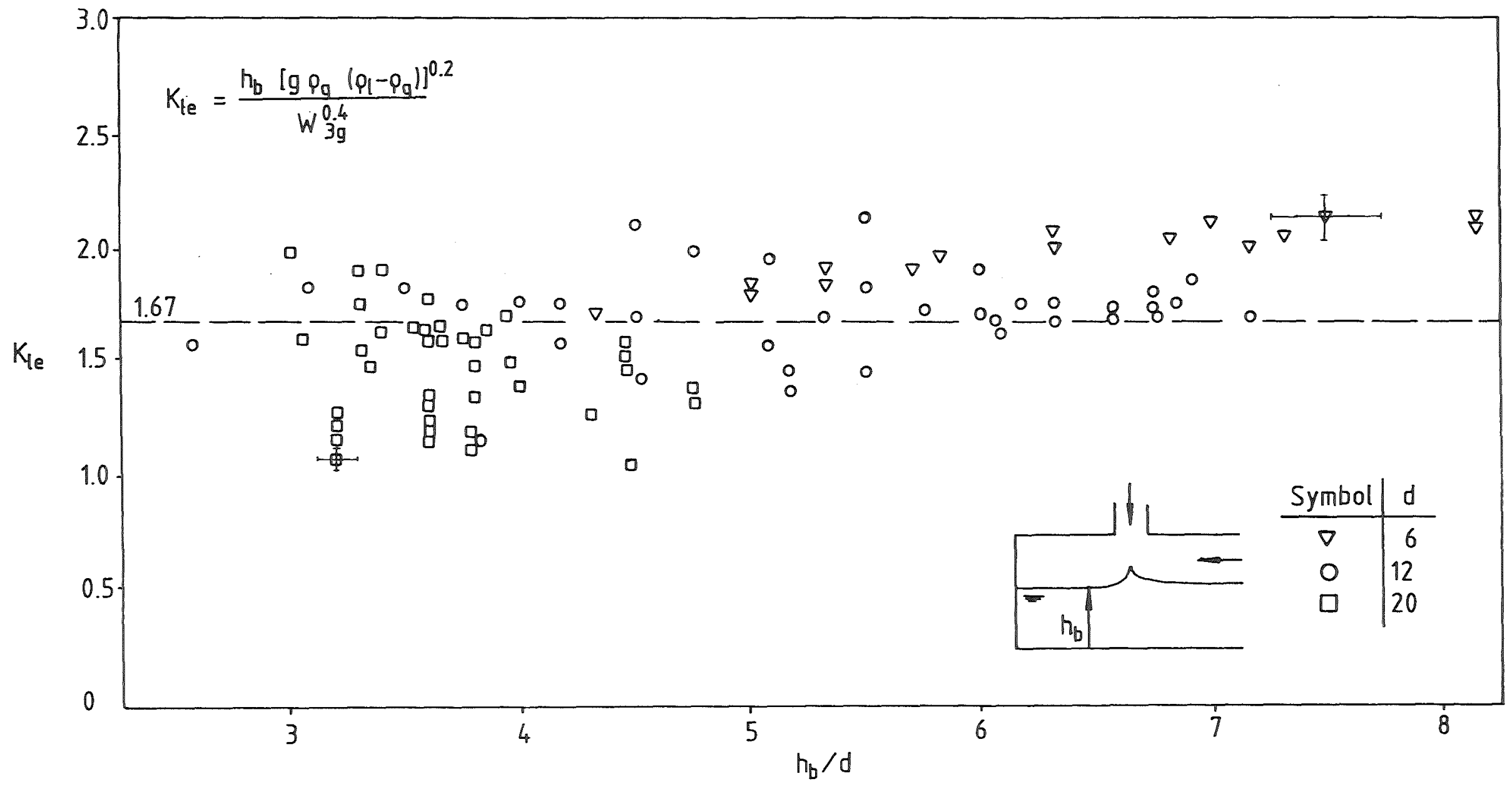

Fig. 24: Beginning of liquid entrainment in upward branches. 


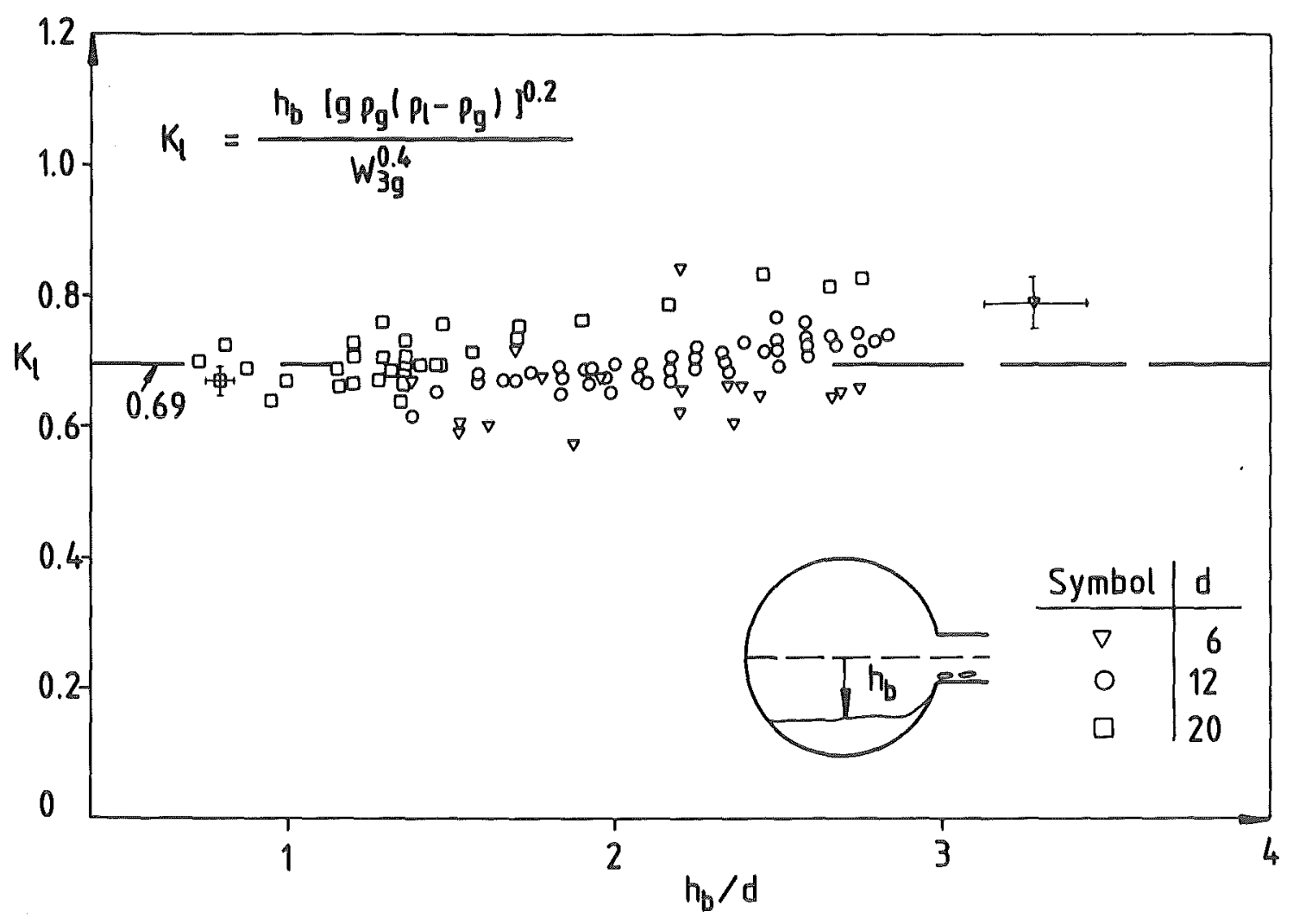

a) Beginning of liquid entrainment

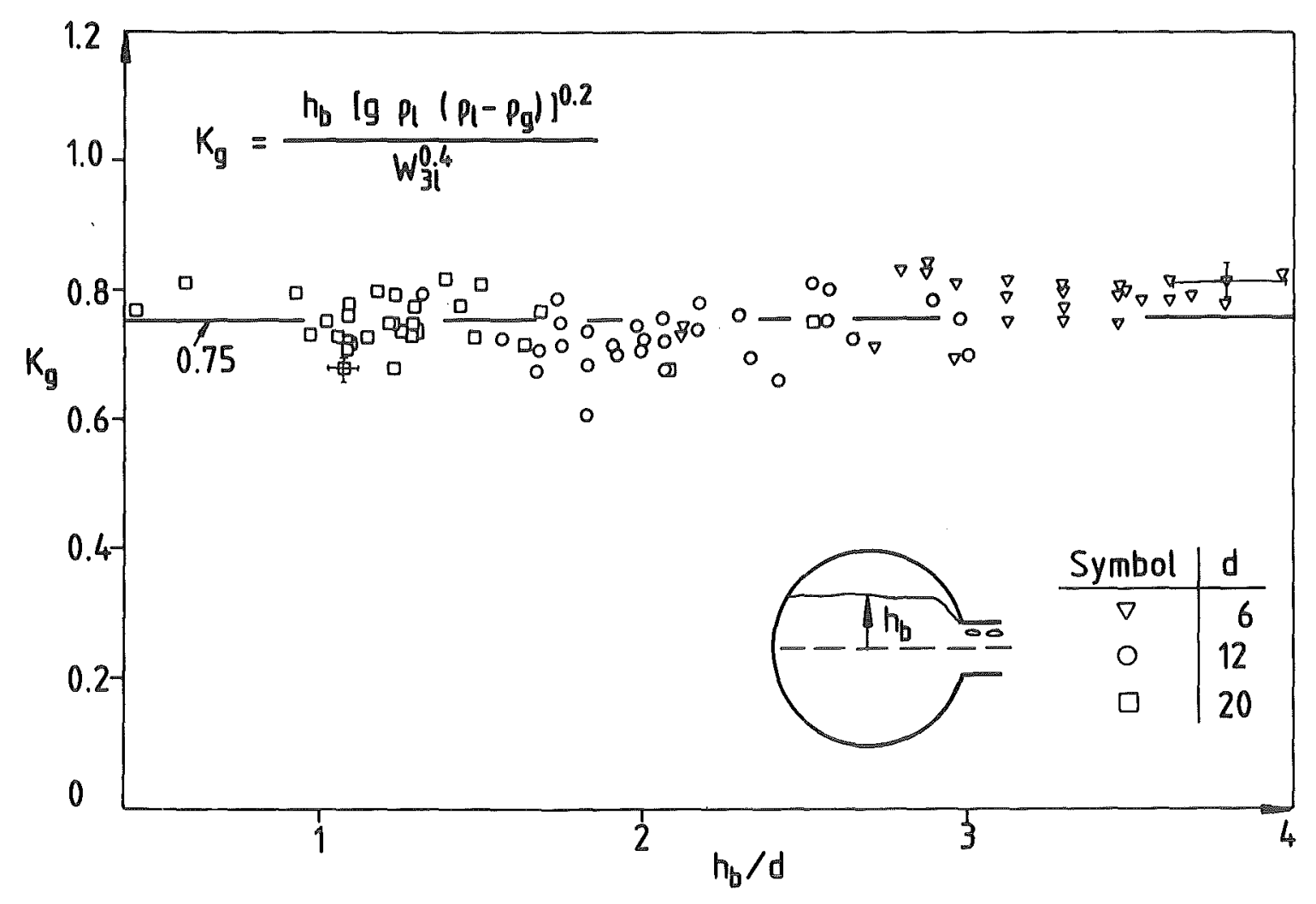

b) Beginning of gas entrainment

Fig. 25: Beginning of entrainment in horizontal branches 
geometries no dependency of $k$ on $h_{b} / d$ is observed.

Eq. (5) was also used to describe the transition from vortex to vortex free Elow in downward branches without superimposed velocities. Results from /17, $18 /$ are shown in Fig. 26 where the parameter $h_{t r}$ represents the liquid level. for the transition. Again, in a reasonable approximation, a constant value of $\mathrm{K}$ was obtained.

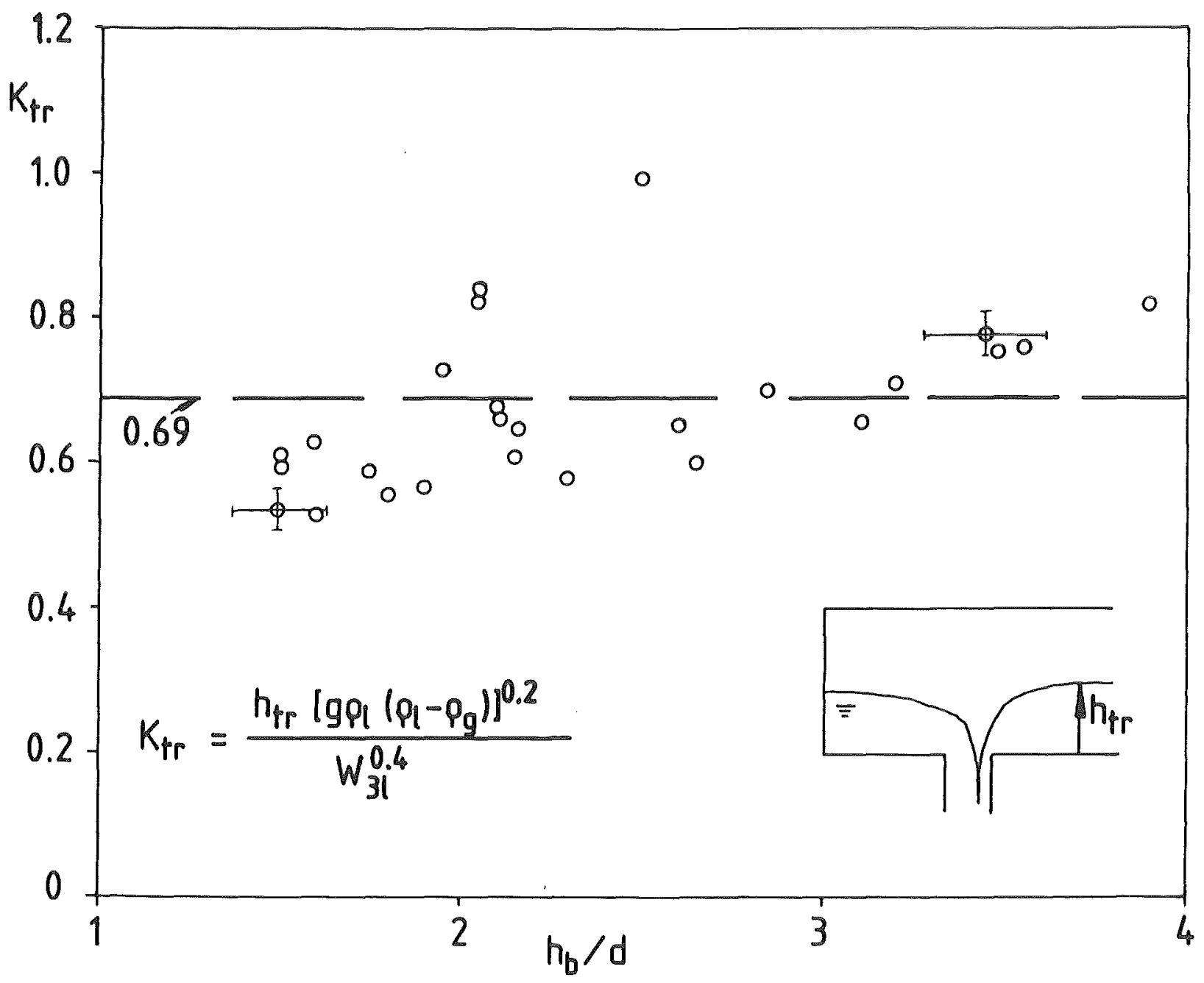

Fig. 26: Transition from vortex to vortrex free flow in downward branches; $\mathrm{d}=12 \mathrm{~mm}, \mathrm{v}_{21}=0$ (data from $\left./ 17,18 /\right)$.

The mean values of $K$ and the corresponding standard deviation $\sigma$ of the experimental points are summarized in Table 3 below. 


\begin{tabular}{|c|c|c|c|c|c|}
\hline \multirow{2}{*}{} & \multicolumn{2}{c|}{ Downward } & Upward & \multicolumn{2}{c|}{ Horizontal } \\
\cline { 2 - 6 } & b.g.e. & Transition & $b .1 . e$. & b.g.e. & $b .1 . e$. \\
\hline $\mathrm{K}$ & 2.00 & 0.69 & 1.67 & 0.75 & 0.69 \\
\hline$\sigma \%$ & 13 & 18 & 16.2 & 5.5 & 5.2 \\
\hline
\end{tabular}

Table 3: Experimental results for the constant $K$ given by Eq. (5).

A general data scatter greater than that corresponding to inaccuracies of measurements is not surprising if the characteristics of the flow field described in Sec. 4 are considered. For downward branches (Sec. 4.1) the instability in the first hoses of gas entering the branch determine the b.g.e. at different liquid levels for identical experiments. Also a considerable indetermination in the liquid level for the transition from vortex to vortex free flow is introduced by strong vortex fluctuations in the flow toward the branch. For upward branches, the vorticity in the continuous gas and the subsequent radial dispersion of the liquid raised from the interface (Section 4.2), cause an initial random arrival of droplets at the branch entrance, which makes it difficult to decide whether the conditions for the b.1.e. have been attained or not. For horizontal branches (Section 4.3) the fluctuations at b.g.e. were lower than in downward branches, and the ascendent water film determining the b.1.e. was not affected by the vorticity of the gas. ThereFore, a data scatter smaller than for the previous geometries was obtained.

To investigate the influence of superimposed velocities on the b.e. data from experiments performed with $\mathrm{v}_{2 \mathrm{~g}}>0$ and $\mathrm{v}_{21}>0$ were used to plot $\mathrm{K}$ as a function of the dimensionless ratios of momentum flux $\rho_{g} v_{2 g}^{2} / \rho_{b} v_{3 b}^{2}$ and $P_{1} v^{2}{ }_{21} / P_{b} v_{3 b}^{2}$, respectively.

Results from $/ 17,18 /$, corresponding to b.g.e. in downward branches with $v_{21} \leqslant 0.36 \mathrm{~m} / \mathrm{s}$, are shown in Fig. 27. The transition from vortex to vortexfree flow described in Section 4.1 (Fig. 10), is very unstable and occurs for relatively small values of $v_{21}$. For higher superimposed liquid velocities the vortex free flow is stable and the constant value $\mathrm{K}=1.17$ was obtained to describe the b.g.e. 


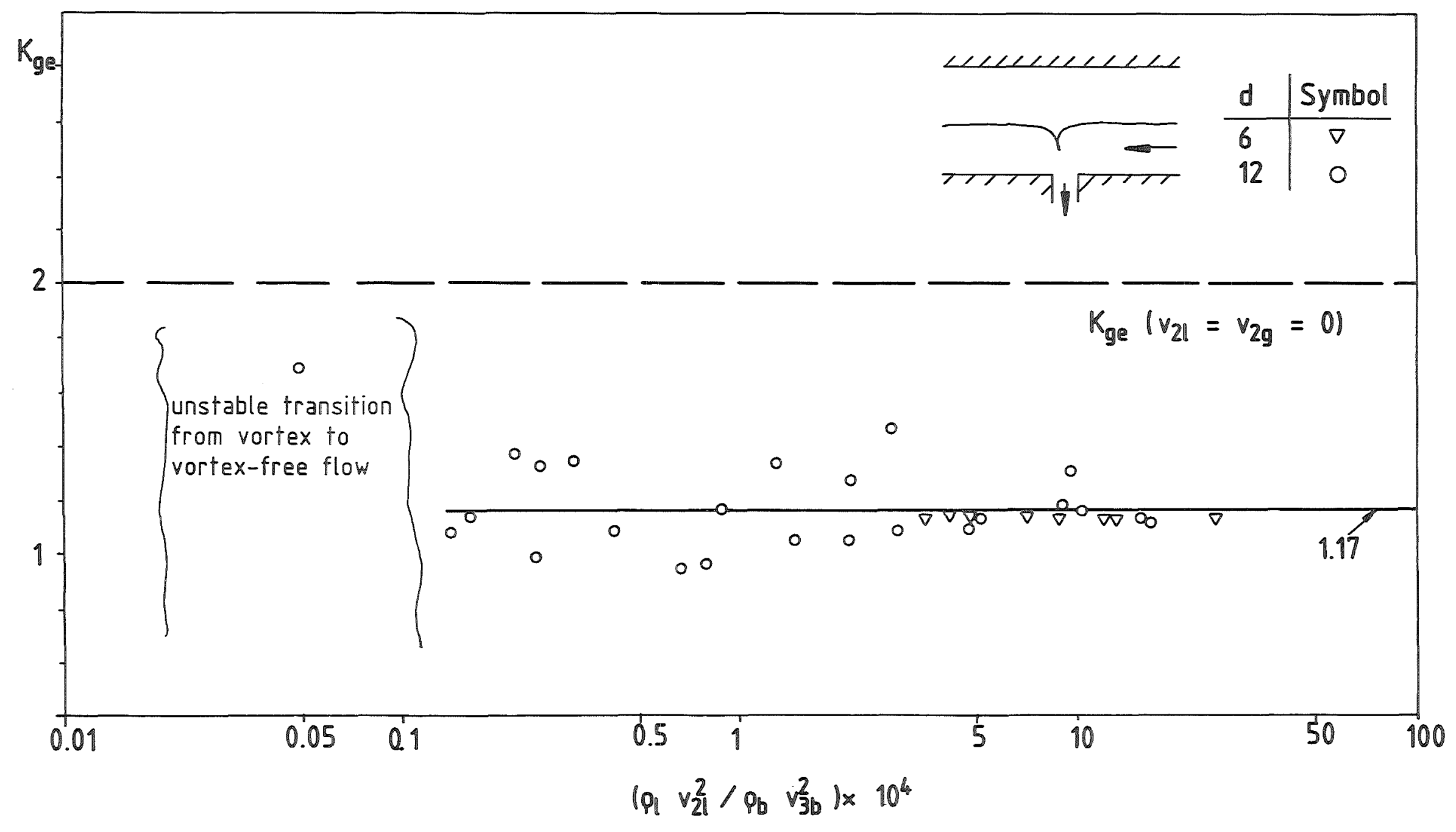

Fig. 27: Beginning of gas entrainment in downward branches with superimposed liquid velocities: $v_{21} \leq 0.36 \mathrm{~m} / \mathrm{s}$ (data from $/ 17,18 /$ ). 
Results corresponding to b.1.e. in upward branches with $v_{2} g \leq 2.1 \mathrm{~m} / \mathrm{s}$ and $v_{21}$ $\leq 0.12 \mathrm{~m} / \mathrm{s}$, are presented in Fig. 28. The gas flow entering the branch was always dominated by vorticity and, in agreement with the analysis in Section 5.3.3, the value of $K$ in Fig. $28 \mathrm{a}$ is observed to decrease for increasing $v_{2 g}$ • Due to the limitation in the maximum value of $v_{2 g}$ imposed by the transition to slug flow (see Section 4.2), a vortex free flow cannot be reached.

As expected, no change in $\mathrm{K}$ is observed for varying $\mathrm{v}_{21}$ (see Fig. 23b). For both flow configurations the present results are in good agreement with previous data from $/ 29 \%$

The results for horizontal branches are presented in Figs. 29-30. In these experiments the ratios of momentum fluxes were one order of magnitude greater than the corresponding values for upward branches.

The curves in Fig. 29 correspond to b.1.e. with $v_{2 g} \leqslant 1.6 \mathrm{~m} / \mathrm{s}$ and $v_{21} \leqslant 0.7$ $\mathrm{m} / \mathrm{s}$. The increasing value of $\mathrm{K}$ with increasing $\mathrm{v}_{2 \mathrm{~g}}$ (Fig. 29a) and $\mathrm{v}_{21}$ (Fig. $29 \mathrm{~b})$ is surprising. It can be attributed to small capilary waves $(\lambda \cong 1 \mathrm{~cm})$ on the interface. In effect, the b.1.e. will be determined by the liquid raised from the crest of the waves and therefore a higher value of the distance $h_{b}$ between the branch axis and the ideally horizontal interface will be registered at b.e.

The results in Fig. 30 correspond to b.g.e. with $v_{2 g} \leq 1.9 \mathrm{~m} / \mathrm{s}$ and $v_{21} \leq 0.5$ $\mathrm{m} / \mathrm{s}$. With the interface far above the branch axis, no influence of surface ripples on the b.g.e. is expected. However, with the high mass flow rates used in these experiments $\left(0.8 \leq w_{11}(\mathrm{~kg} / \mathrm{s}) \leq 11\right)$, possible fluctuations in the liquid flow may favor the occurrence of the initial intermittent thin hoses of gas determining the b.g.e., which explains the slightly increasing value of $\mathrm{K}$ obtained with $\mathrm{v}_{21}>0$ (Fig. $30 \mathrm{~b}$ ). The experiments with $\mathrm{v}_{2 \mathrm{~g}}>0$ were performed with $v_{11}=0$, and therefore no change in $K$ is observed ( $F i g$. $30 a)$.

The solid line curves in Figs. $28 \mathrm{a}, 29$ and $30 \mathrm{~b}$ were qualitatively drawn through the experimental points.

The results in this section show that relatively low superimposed velocities $\left(v_{2 g} \leqslant 1 \mathrm{~m} / \mathrm{s} ; v_{21} \leqslant 0.1 \mathrm{~m} / \mathrm{s}\right)$ have a very little influence on the values of $\mathrm{K}$ 


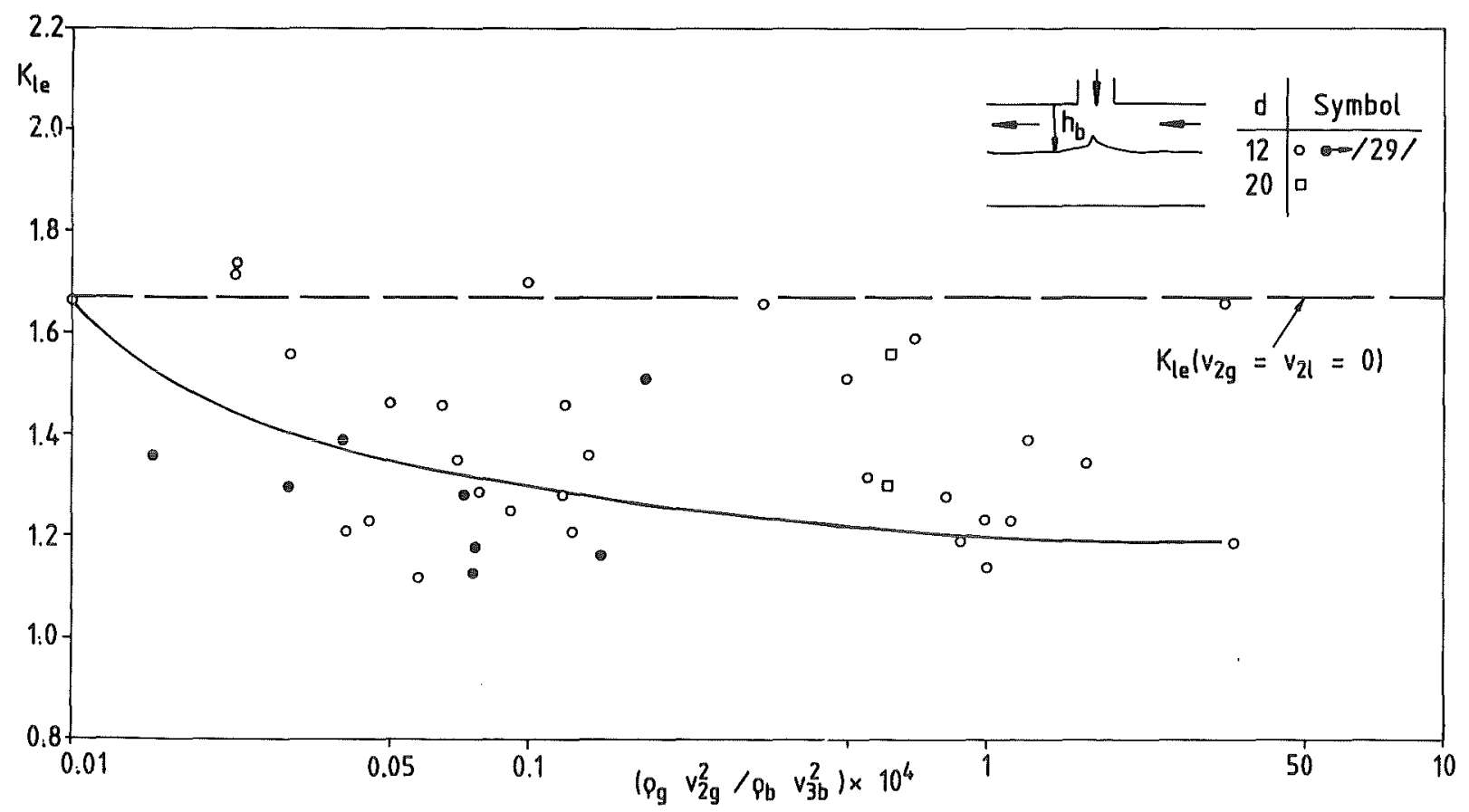

a) $v_{2 g} \leq 2.1 \mathrm{~m} / \mathrm{s} ; v_{21}=0$.

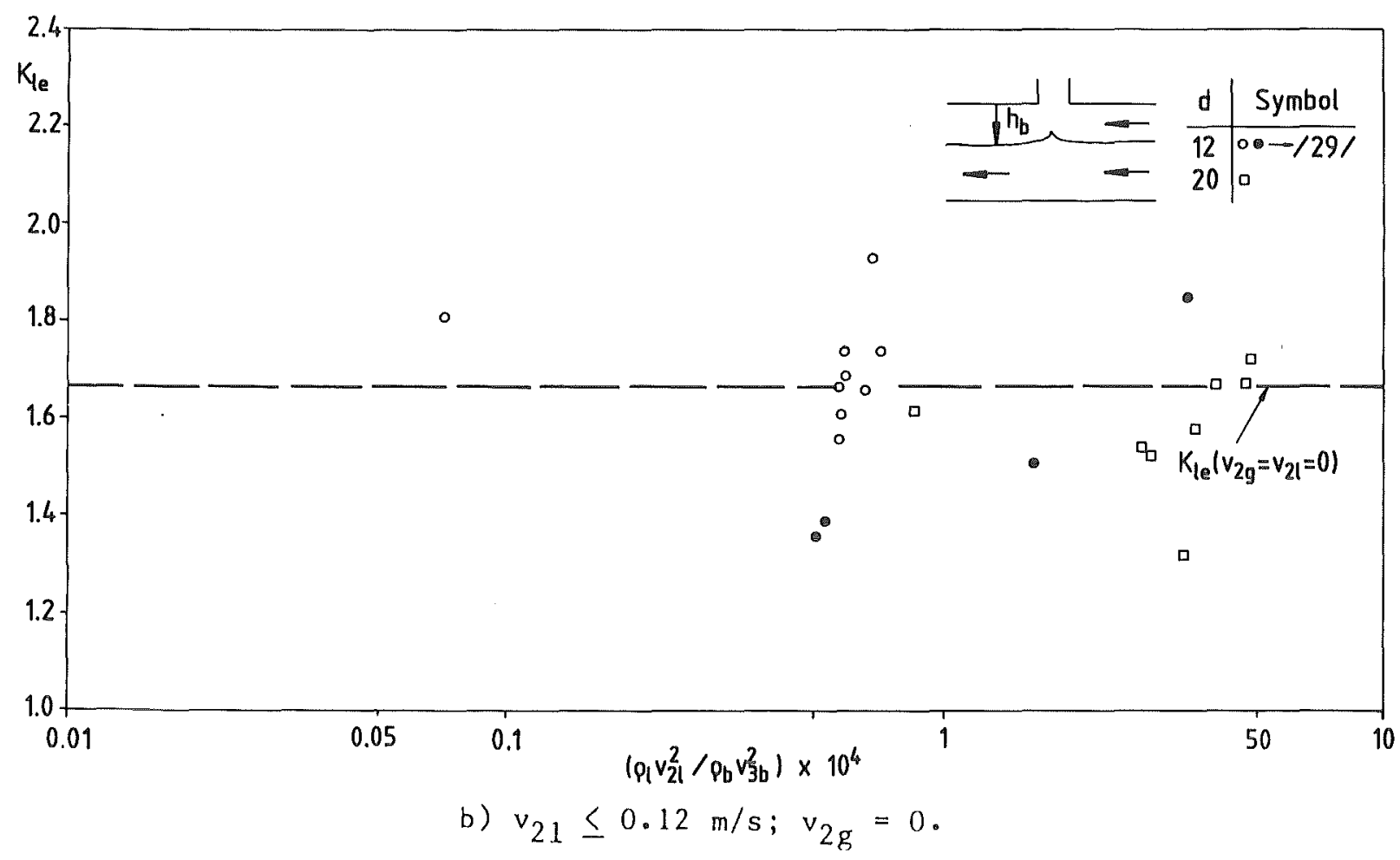

Fig. 28: Beginning of liquid entrainment in upward branches with superimposed velocities. 


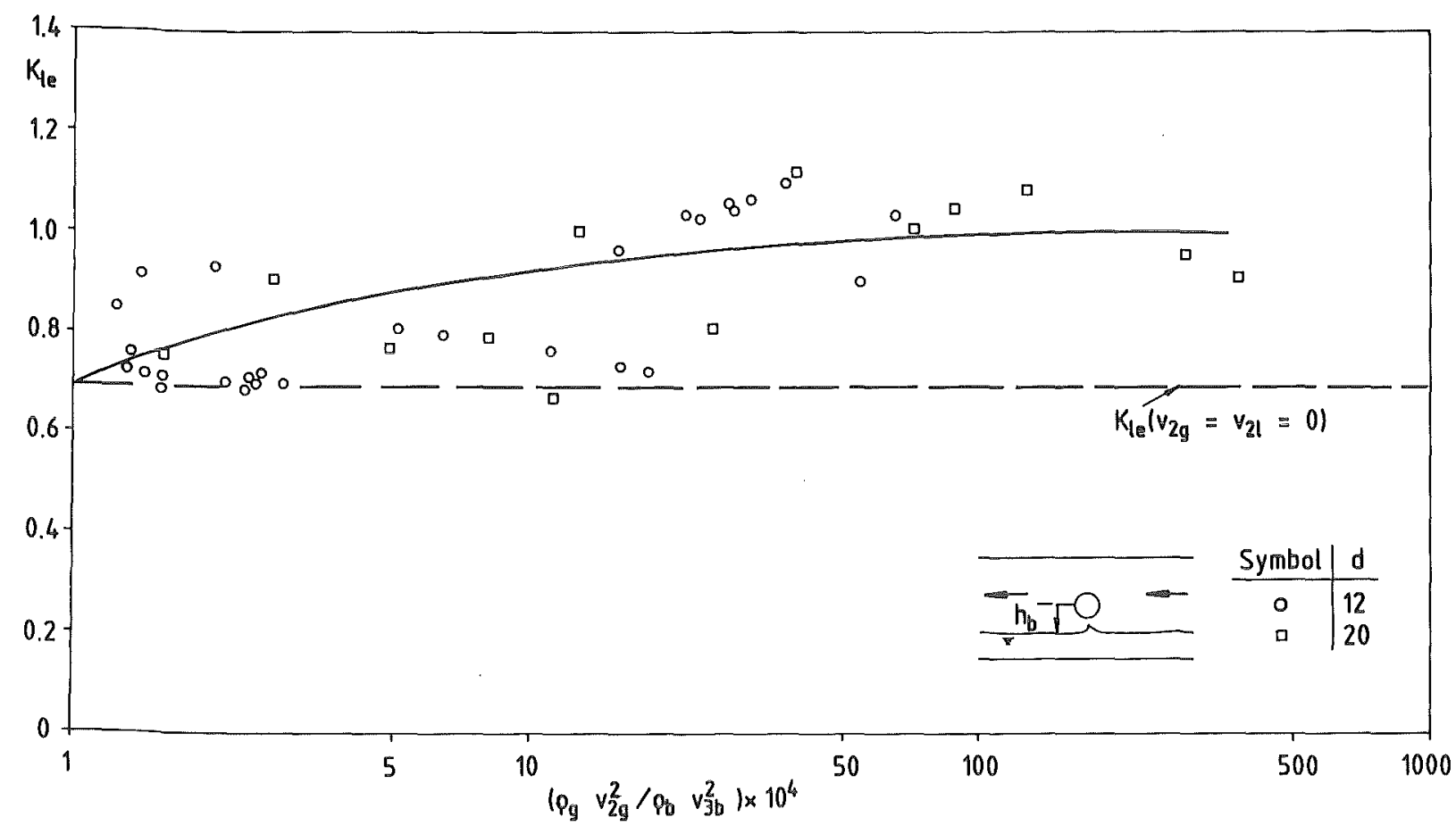

a) $v_{2 g} \leq 1.6 \mathrm{~m} / \mathrm{s} ; v_{21}=0$

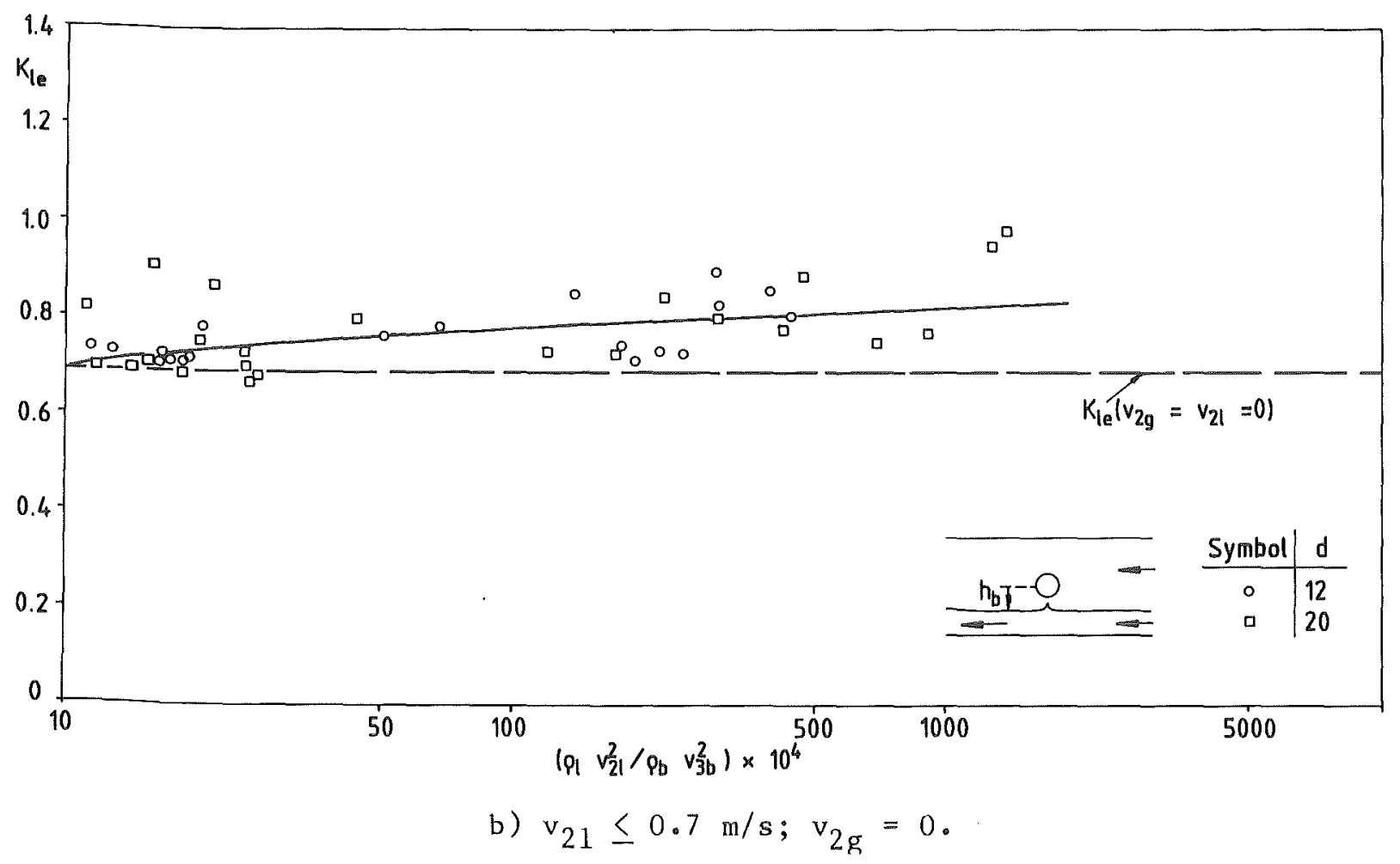

Fig. 29: Beginning of liquid entrainment in horizontal branches with superimposed velocities. 


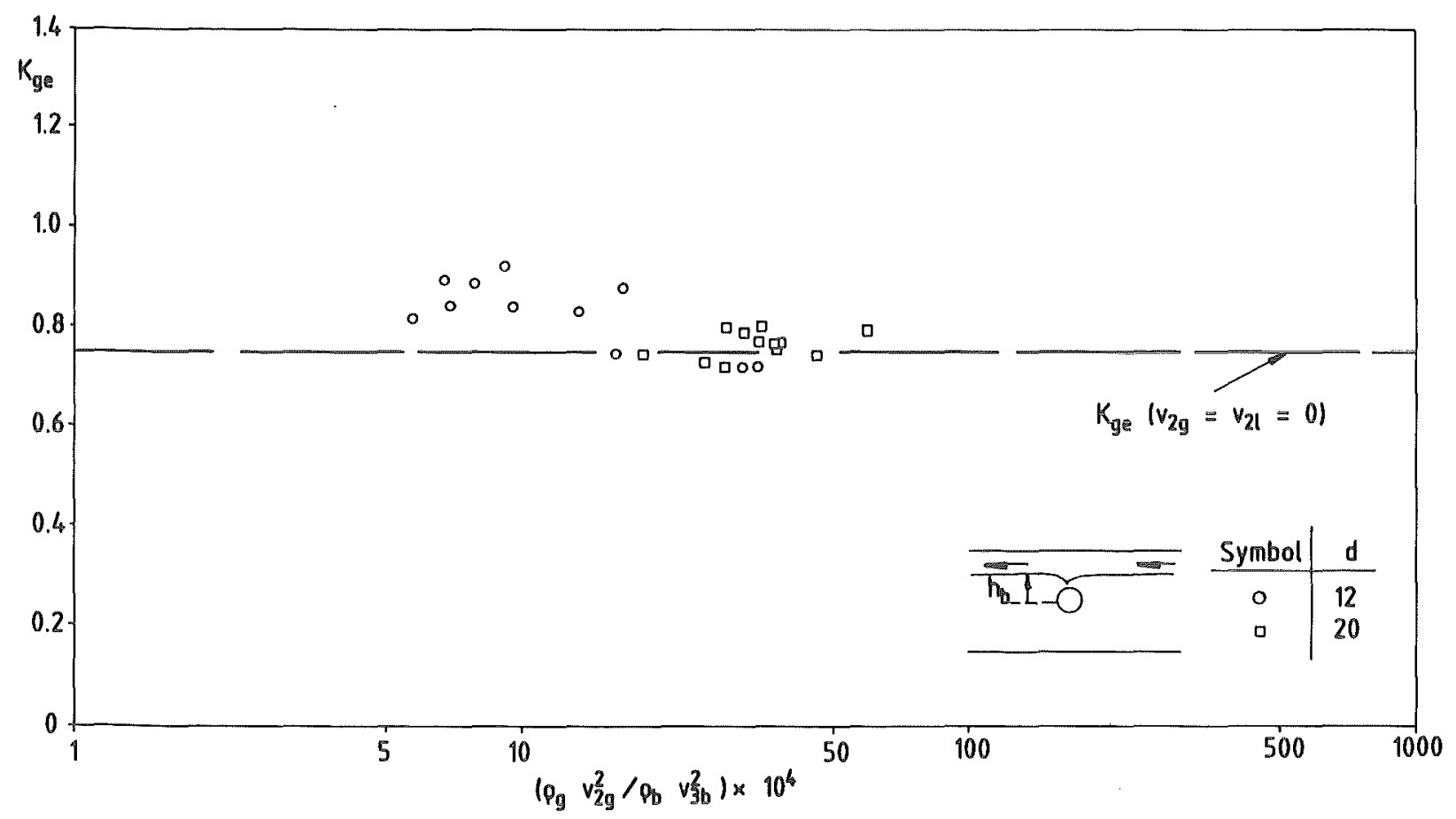

a) $v_{2 g} \leq 1.9 \mathrm{~m} / \mathrm{s} ; v_{21}=0$

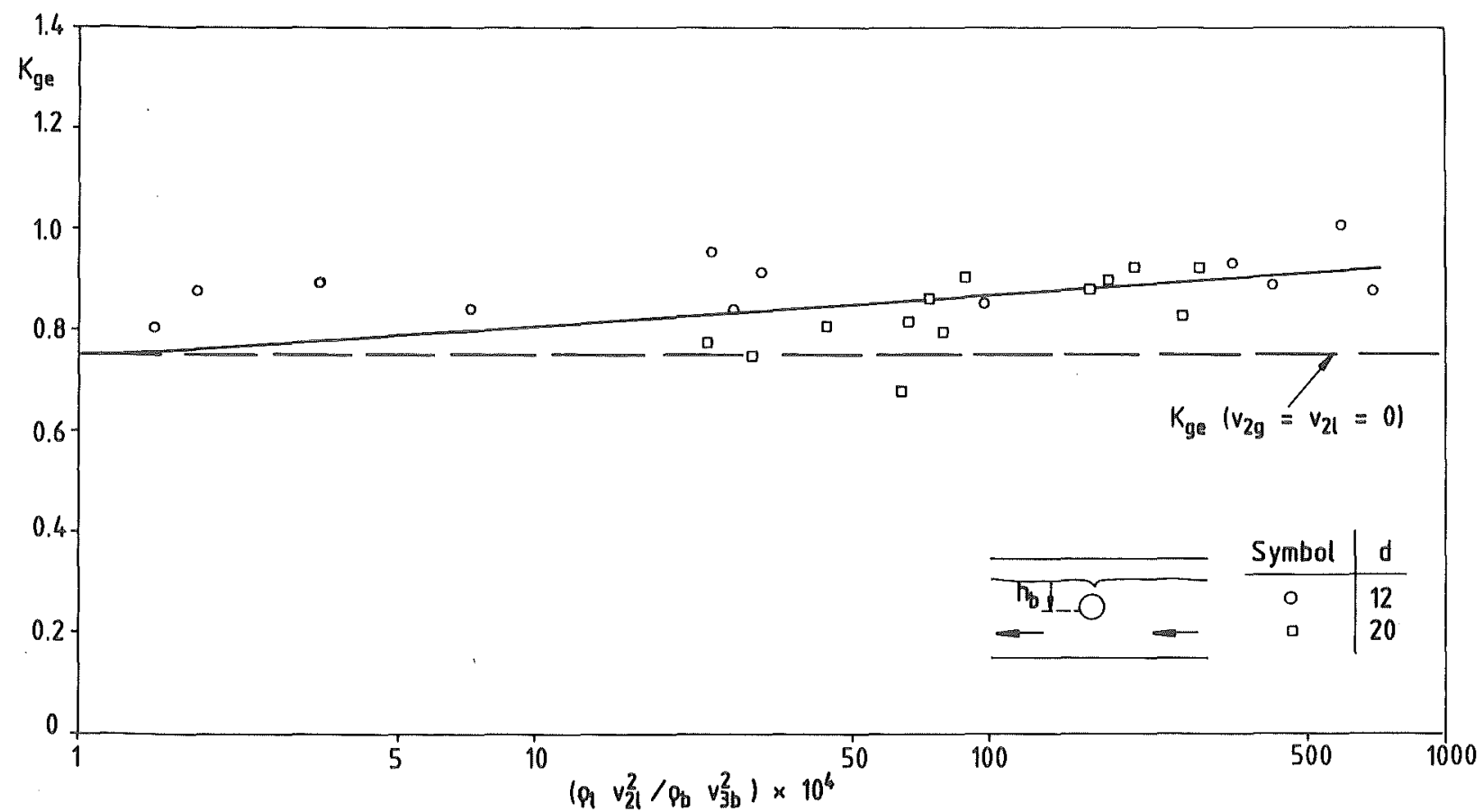

b) $v_{21} \leq 0.5 \mathrm{~m} / \mathrm{s} ; v_{2 g}=0$

Fig. 30: Beginning of gas entrainment in horizontal branches with superinposed velocities 
for b.e. Therefore, in experiments without superimposed velocities, for which always $v_{1} \ll v_{3}$, no significant change of $\mathrm{K}$ can be expected if the inflow is reduced to zero. In such a case the values of $K$ in Table 3 could be used together with Eq. (5) to determine the b.e. in small breaks at the bottom, the top or the side of large reservoirs, in which the momentum of the fluid is negligible. 
6. Branch Quality and Mass F1ux

6.1 Single Phase Flow

To determine the single phase branch mass flux as a function of the total pressure difference $\Delta \mathrm{P}_{1-34}$, the influence of the flow parallel to the main pipe is neglected and two zones in the branch flow are distinguished (Fig. 31): an acceleration zone a vena contracta develops followed by a friction zone extending up to the end of the branch.

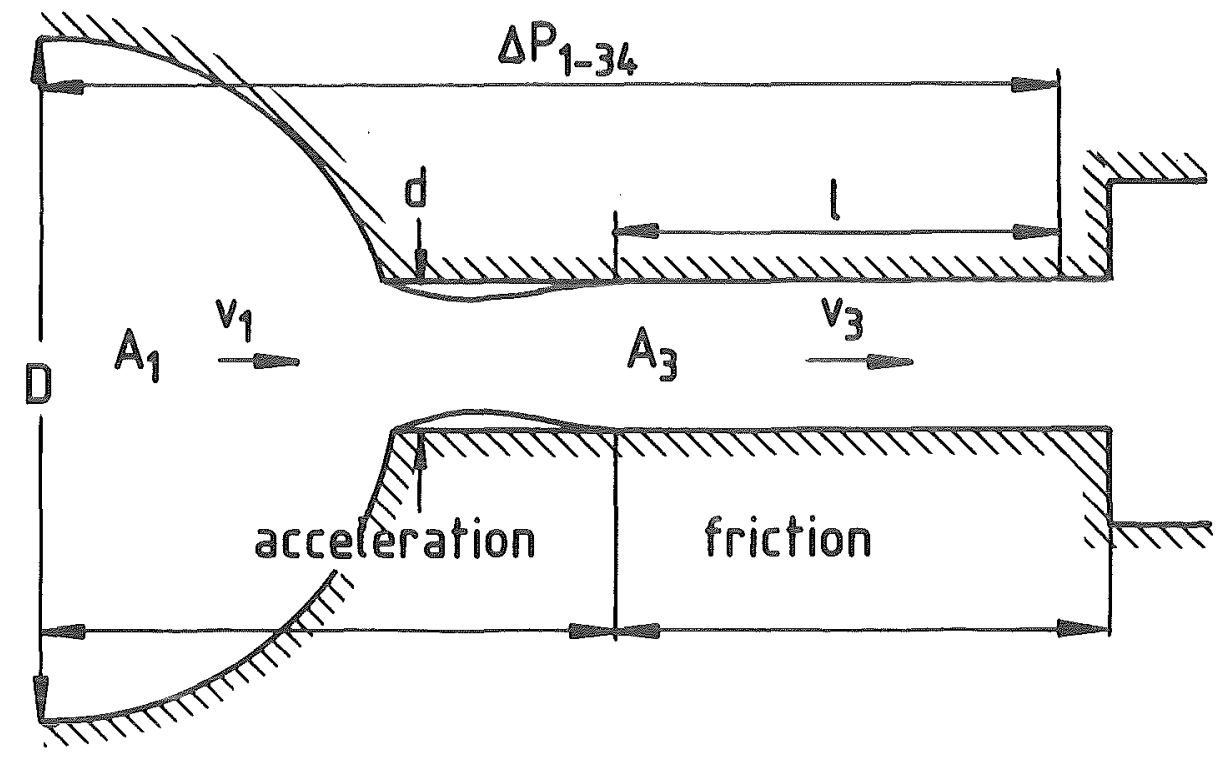

Fig. 31: Acceleration and friction zones in the branch flow.

The total pressure difference $\Delta P_{1-34}$ can be written as follows

$$
\Delta P_{1-34}=\Delta P_{\text {rev }}+\Delta P_{\text {irrev }}
$$

The term $\Delta \mathrm{P}_{\text {rev }}$ represents the total reversible pressure drop due to the acceleration of the fluid and is given by:

$$
\Delta P_{\text {rev }}=\frac{1}{2 \rho}\left(G_{3}^{2}-G_{1}^{2}\right)
$$

For a diameter ratio d/D $\ll 1\left(G_{1} \ll G_{3}\right)$ Eq. (29) reduces to:

$$
\Delta P_{\text {rev }}=\frac{1}{2} \frac{G_{3}^{2}}{\rho}
$$


The term $\Delta P_{\text {irrev }}$ in $E q \cdot(28)$, represents the pressure loss due to the irreversible processes occurring in the acceleration and friction zones:

$$
\Delta P_{\text {irrev }}=\left(k_{c}+\frac{4 l}{d} f\right) \frac{1}{2} \frac{G_{3}^{2}}{\rho}
$$

where $K_{c}$ and $f$ are empirical coefficients corresponding to the acceleration and frictional zones, respectively. The length 1 is defined in Fig. 31 .

For sudden contractions with sharp edged entrance and $A_{3} / A_{1} \rightarrow 0$, Weisbach $130 /$ proposes the value 0.5 for the coefficient $k_{c}$. For the friction coefficient $F$ many correlations are available. In particular, a frequently used expression valid for $3 \times 10^{3} \leq \operatorname{Re} \leq 3 \times 10^{6}$, was given by Drew et al $/ 31 /$ :

$$
E=0.0014+0.125 \operatorname{Re}^{-0.32}
$$

With the following typical values from the present experiments: $1=50 \mathrm{~mm}$; $=12 \mathrm{~mm}: \operatorname{Re}=7.5 \times 10^{4}$ it results: $4 \mathrm{ff} / \mathrm{d} \simeq 0.1$. Thus, Eq. (31) gives

$$
\Delta P_{\text {irrev }} \simeq 0.3 \frac{G_{3}^{2}}{\rho}
$$

By substituting Eq. (33) and Eq. (29) into Eq. (28), the total pressure difference through the branch is:

$$
\Delta P_{1-34}=0.8 \frac{G_{3}^{2}}{\rho}
$$

which finally gives for the incompressible branch mass flux:

$$
G_{3}=1.12 \sqrt{\rho \Delta P_{1-34}}
$$

In practice, however, an expression of the form

$$
G_{3}=7 \sqrt{\rho \Delta P_{1-34}}
$$

was used with the flow coefficient $\}$ determined experimentally.

Although recent experiments have revealed $\}$ to be a function of Reynolds number and boundary roughness (compare e.g. Streeter /32/), these influences can in practice be ignored and only a weak dependency on the geometry could be expected. 
The forgoing treatment can be extended to compressible fluids by multiplying the right hand side of Eq. (36) with the expansion coefficient $\varepsilon$ for gases, which gives:

$$
G_{3}=\left\{\varepsilon \sqrt{\rho \Delta P_{1-34}}\right.
$$

with $\varepsilon$ as given in VDI $2040 / 33 /$ :

$$
\varepsilon=1-\left[0.3707+0.3184\left(\frac{d}{D}\right)^{2}\right]\left(1-\left(\frac{P_{3}}{P_{1}}\right)^{1 / \gamma}\right]^{0.935}
$$

where the isentropic coefficient is $\gamma=0.4$ for air.

Eq. (37) was used to determine the flow coefficient 3 for water and subcritical air flow through branches of different diameters. The results are presented in Fig. 32 as a function of Re.

In the range covered by the experiments no significant dependency of 3 on $\operatorname{Re}$ can be inferred. A small difference exists between the results for air and water flows; however, a mean value valid for single phase flow was taken for each diameter, as summarizd in Table 4 below

\begin{tabular}{|c|c|c|c|c|}
\hline$d(\mathrm{~mm})$ & 6 & 8 & 12 & 20 \\
\hline$\}$ & 0.905 & 0.949 & 0.990 & 0.968 \\
\hline$\sigma(\%)$ & 8 & 17 & 6 & 16 \\
\hline
\end{tabular}

Table 4: Subcritical single phase flow coefficient for branches of different diameters.

The different values of $\}$ obtained for different branch diameters can be attributed to small differences of the branch entrance geometry (roundness for instance). 


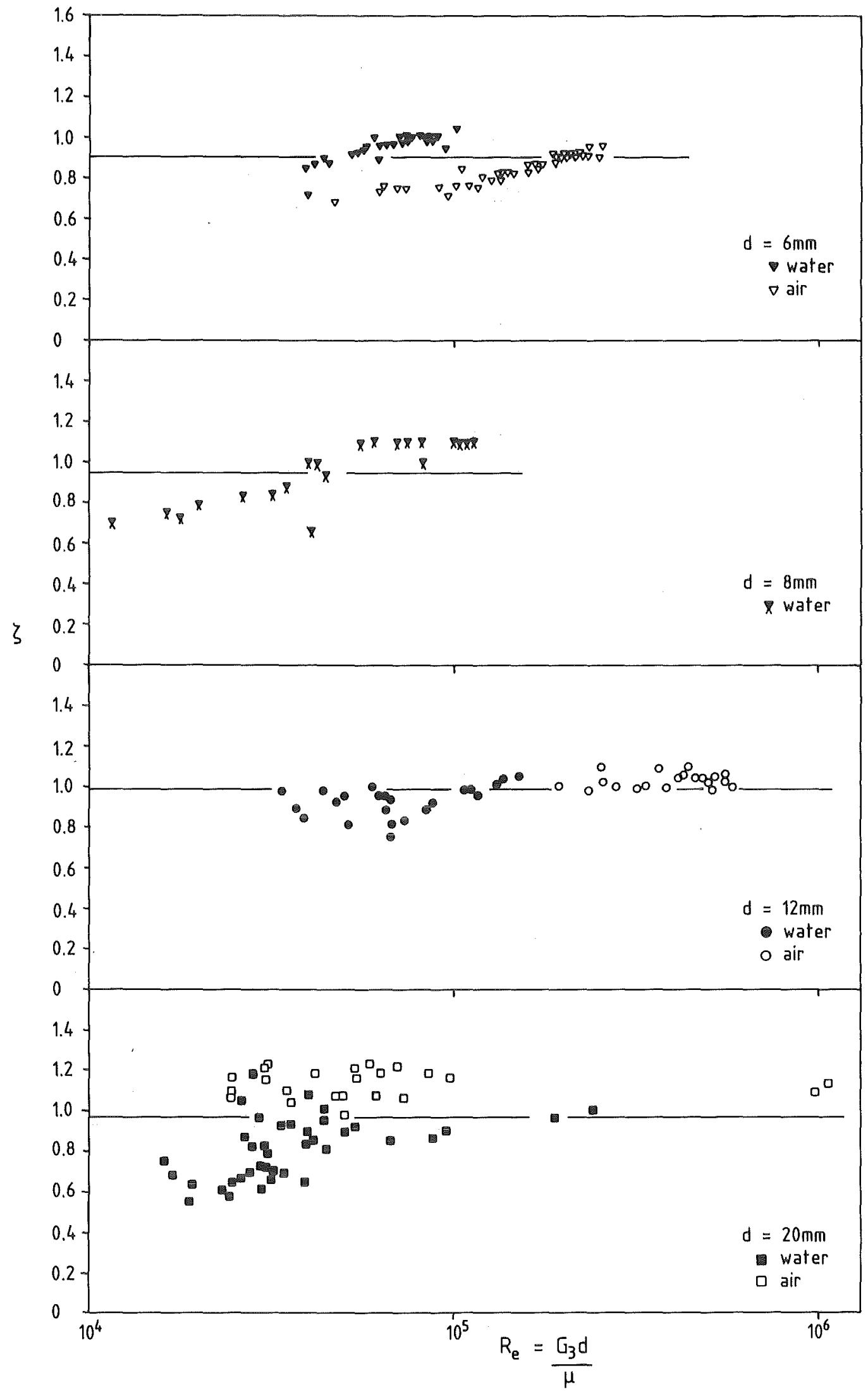

Fig. 32: Flow coefficients for single phase subcritical flow in branches with different diameters, as a function of Reynolds number. 


\subsection{Two Phase Flow}

\section{2 .1 Generalized Representation of Data}

To present the data for liquid or gas entrainment the procedure previously used $(/ 17,18 /)$ was applied: the measured $h$ and $G_{3}$ were normalized with $h_{b}$ an $G_{3 b}$ respectively, which were calculated with the $P_{1}$ and $\Delta P_{1-34}$ measured during entrainment. Since at b.e. a single phase flow can be assumed in the branch, the parameter $G_{3 b}$ was calculated with $E q$. (37) rewritten as follows:

$$
G_{3 b}=\left\{\varepsilon \sqrt{\rho_{6} \Delta P_{1-34}}\right.
$$

A similar expression is valid for $W_{3 b}$ :

$$
\omega_{36}=\left\{\varepsilon A_{3} \sqrt{P_{5} \Delta P_{1-34}}\right.
$$

where $A_{3}$ is the branch cross-sectional area. By substitution of Eq. (40) into Eq. (5), the parameter $h_{b}$ is given by:

$$
h_{5}=\frac{K\left(\zeta \varepsilon A_{3}\right)^{0.4}}{g^{0.2}}\left(\frac{\Delta P_{1}-34}{\rho_{e}-\rho_{g}}\right)^{0.2}
$$

\section{$6.2 .2 \quad$ Experimental Results \\ 6.2 .2 .1 Downward Branch}

For the normalized representation of data for g.e. with vortex and vortexfree flow, the parameter $h_{b}$ ( $\left.E q . ~(41)\right)$ was calculated with the value of $k$ for b.g.e. in the corresponding flow configuration. Thus, for entrainment with vortex flow the value $K=2$ from Table 3 was used, because it corresponds to b.g.e. with vortex flow. However, for vortex free entrainment, observed when $v_{21}>0$ or when the transition from vortex to vortex free flow with $v_{21}=0$ has occurred, $h_{b}$ was calculated with the value $k=1.17$ from Fig. 27 corresponding to vortex free b.g.e.

Results including data from /17, $18 /$ are presented in Figs. 33-34. Various symbols are used to distinguish between experiments with and without superimposed velocities. The procedure used to fit the data in Fig. 33 is presented in section $6.2 \cdot 3.2$. 


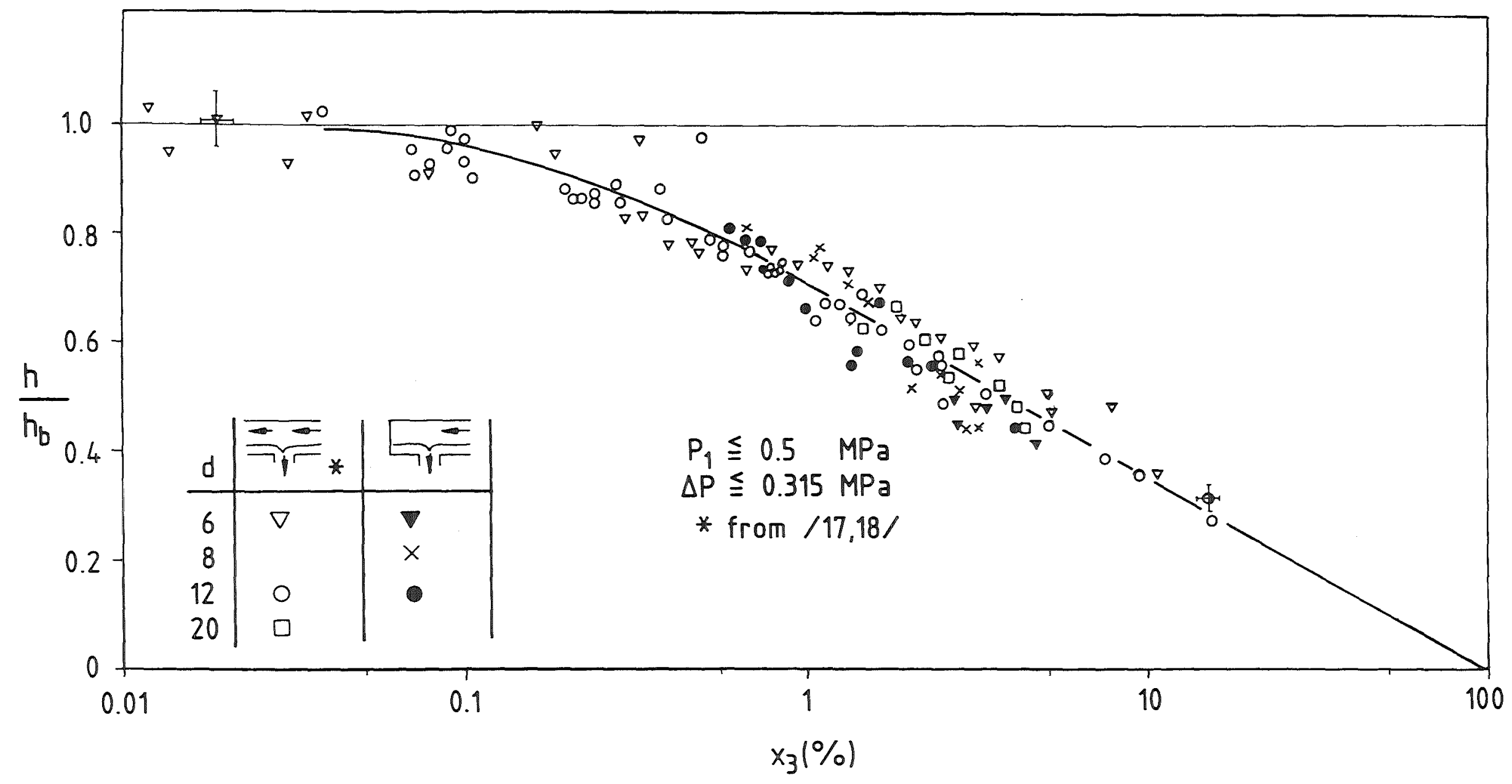

Fig. 33: Gas entrainment in downward branches: dimensionless interface level $\mathrm{h} / \mathrm{h}_{\mathrm{b}}$ as a function of the branch quality $\mathrm{x}_{3}$. 
The curve in Fig. 34 was qualitatively drawn through the experimental points.

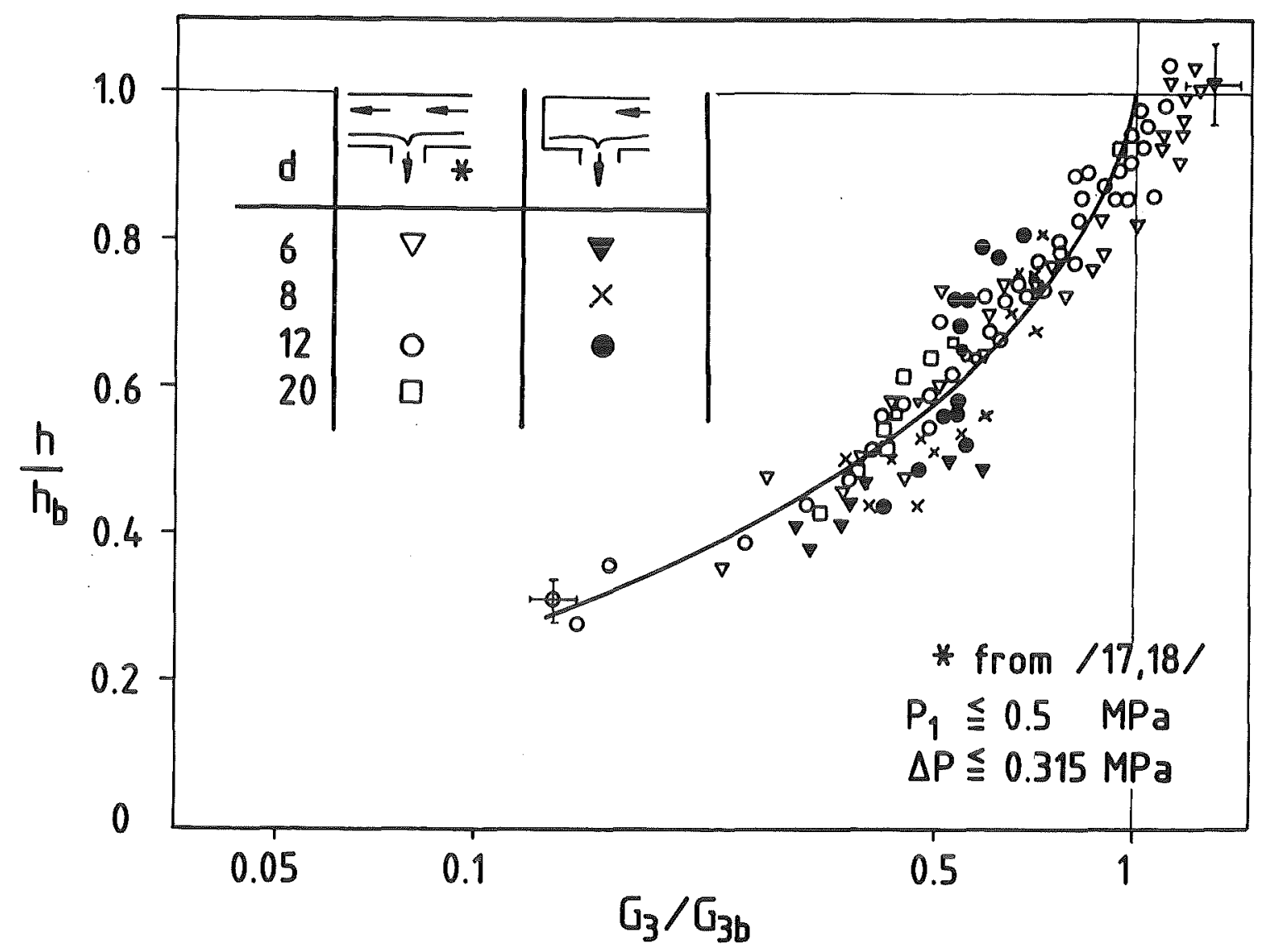

Fig. 34: Gas entrainment in downward branches: dimensionless interface level $h / h_{b}$ as a function of the normalized branch mass $f 1 u x G_{3} / G_{3 b}$.

\section{2 .2 .2 Upward Branch}

Figure 35 shows results for $v_{2 g}=0(K=1.67)$. The solid line curve in Fig. $35 a$ is a qualitative fitting of the data: Starting at $h / h_{b}=1$, and for decreasing values of $h / h_{b}$, the change in $x_{3}$ is relatively small until a sudden decrease occurs at $h / h_{b} \simeq 0.6$ which corresponds to the transition from stratified to slug flow in the test section. The slow decrease of $x_{3}$ during stratified flow is due to the radial dispersion of droplets, produced by the strong vorticity of the gas described in Section 4.2 which avoids the water to reach the branch entrance. Qualities about $100 \%$ determine the value $G_{3} / G_{3 b} \simeq 1$ shown in Fig. 35b. A strong increase of $G_{3} / G_{3 b}$ occurs when the slug flow regime is reached. 
Figure 35 contains some test points with critical branch flux for which $h_{b}$ and $G_{3 b}$ were calculated with the critical pressure difference $\Delta P_{1-34}=P_{1}-$ $\mathrm{P}_{34}$ crit $\simeq 0,47 \mathrm{P}_{1} ;$ no significant influence is observed. The criterion used to draw the dashed curve in Fig. 35 a will be explained in Section 6.2.3.3.

For a typical value $\mathrm{x}_{3}=0,99$ (Fig. 35 a) maximal errors of $0.27 \%$ for $\mathrm{x}_{3}$ and $7.4 \%$ for $G_{3} / G_{3 b}$ can be expected (see Appendix Al).

A data scatter, greater than that introduced by measurement errors, is due to the fluctuations in the entrainment produced by surface waves

in the various flow regimes (see Section 4.2).

According to these results for 1.e. in upward branches, it may be justified to assume that only gas flows through the branch as long as a stratified flow prevails in the main pipe.

\section{2 .2 .3 Horizontal Branch}

As previously, the distance to the interface was measured from the branch axis, however, for liquid levels below this axis, h was considered to be negative; thus, points with $h>0$ correspond to g.e. and those with $h \leq 0$ to 1.e. Figure 36 shows the normalized interface level in the range $-1 \leq h / h_{b} \leq$ 1 as a function of the measured quality. The data for different $\triangle \mathrm{P}_{1-34}$ and branch diameters, are again well fitted by a single curve. With a logarithmic scale for the quality, the value $\mathrm{x}_{3}=0$ is at minus infinite on the horizontal axis; however, in a good approximation, the curve is a straight line in a very wide range of qualities. The criterion used to fit the data will be explained in the next section.

Figure 37 shows the dependency of $h / h_{b}$ on $G_{3} / G_{3 b}$. The very different values of $\mathrm{C}_{3 \mathrm{~b}}$ for liquid and gas entrainment (see Eq. (39)) determine the discontinuity observed at $h=0$. 


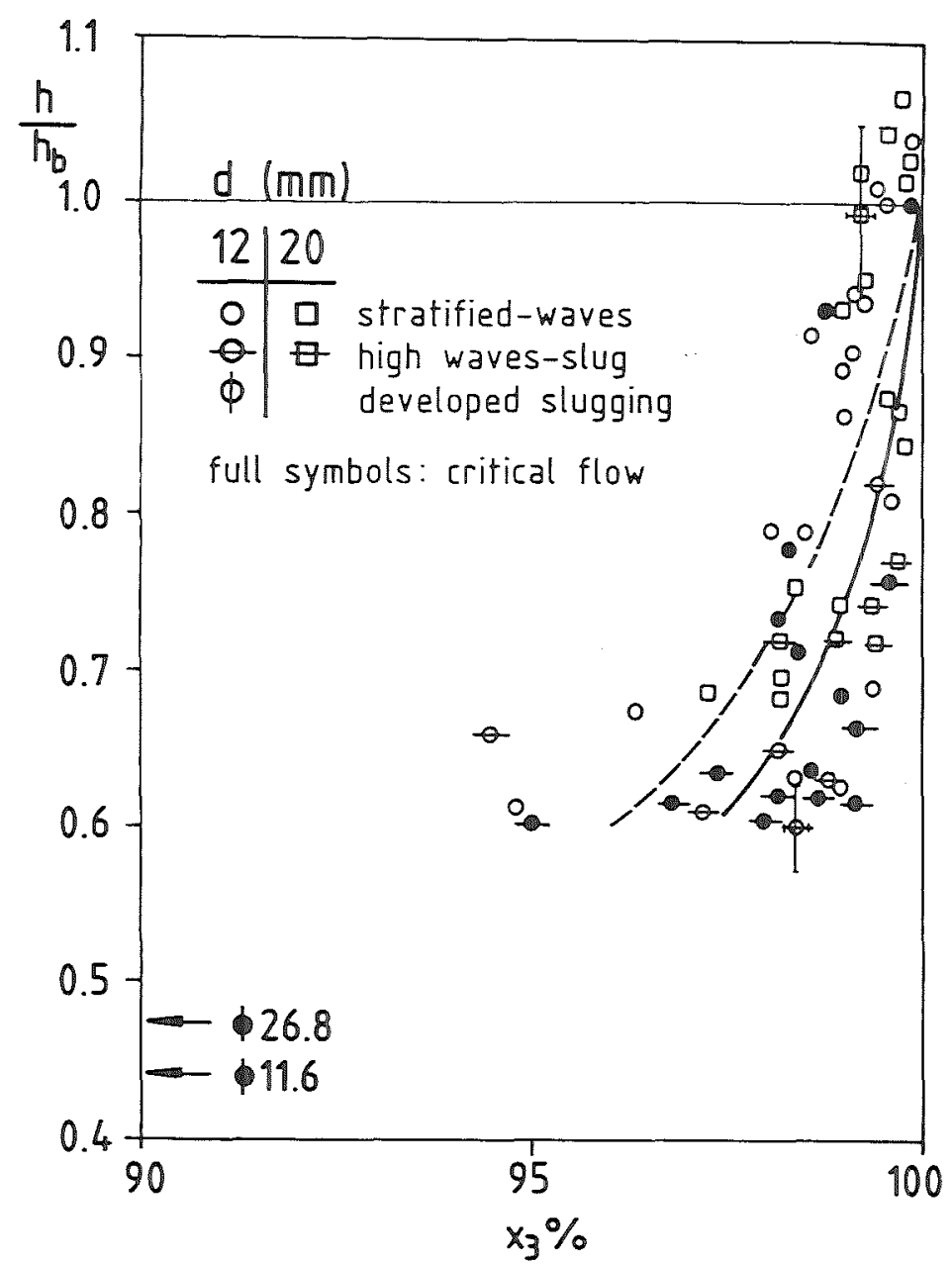

a) dimensionless interface level $\mathrm{h} / \mathrm{h}_{\mathrm{b}}$ as a function of the branch quality $\mathrm{x}_{3}$

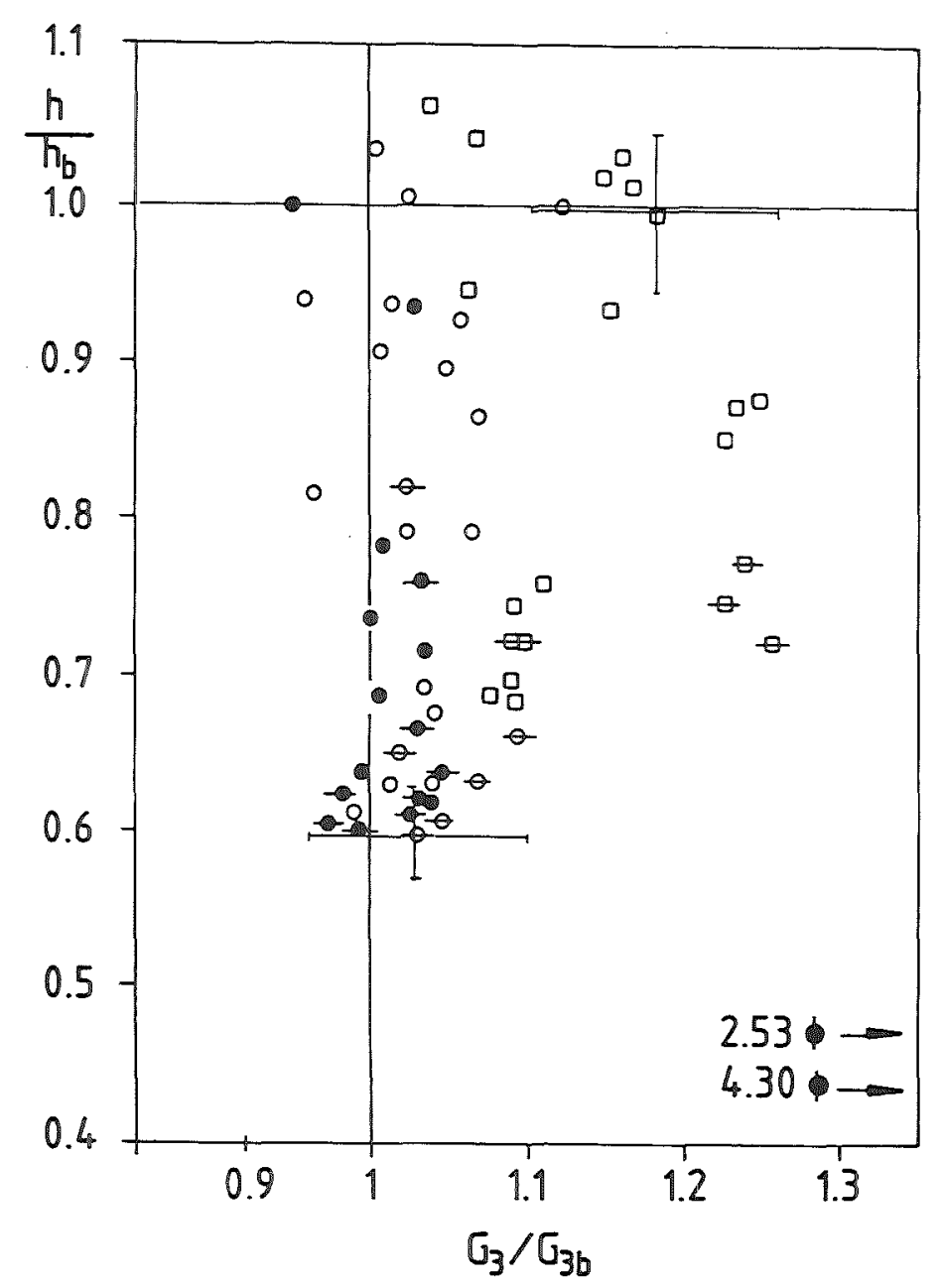

b) dimensionless interface level $\mathrm{h} / \mathrm{h}_{\mathrm{b}}$ as a function of the normalized branch mass flux $\mathrm{G}_{3} / \mathrm{G}_{3} \mathrm{~b}$ 


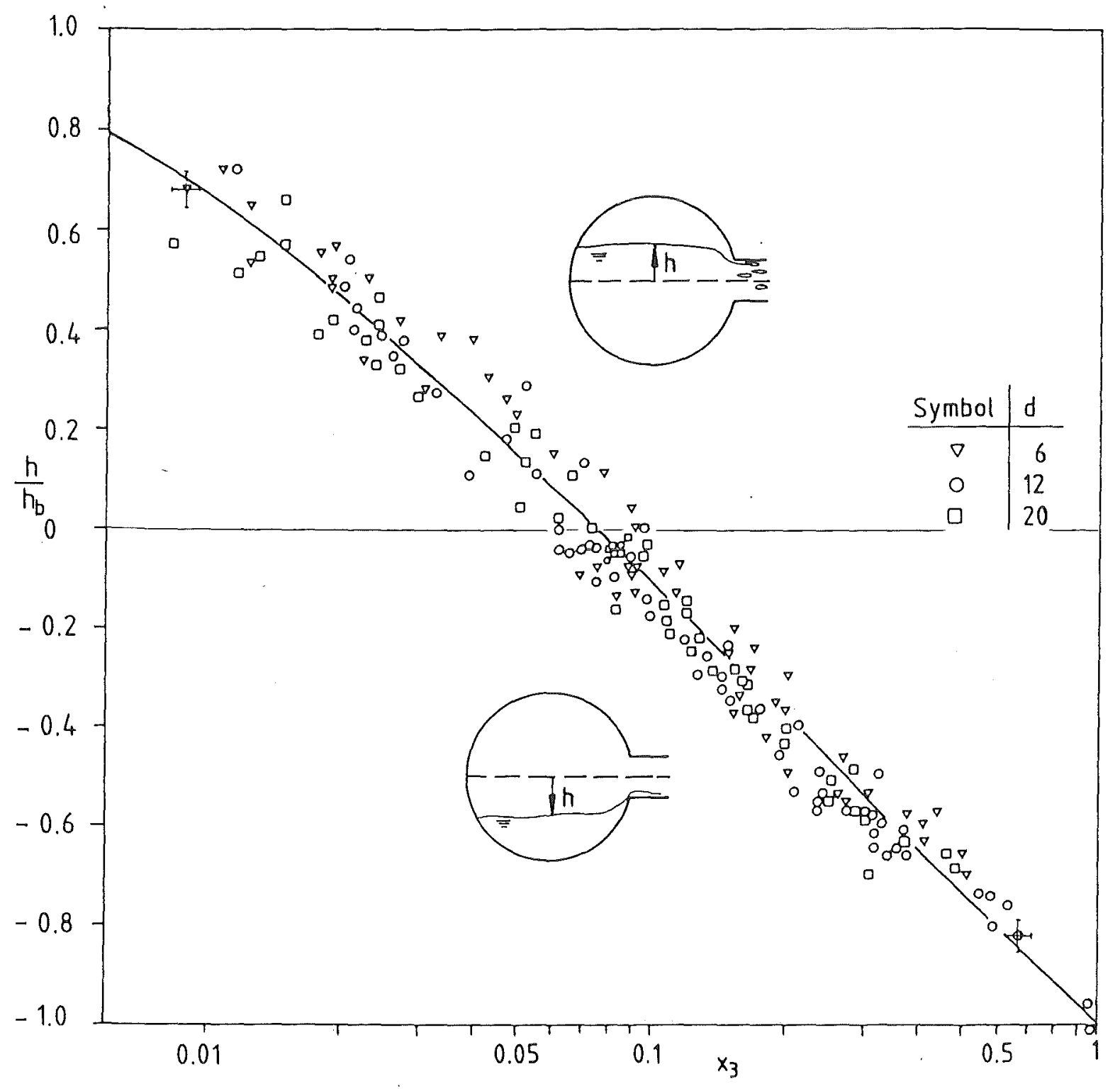

Fig. 36: Gas and liquid entrainment in horizontal branches: dimensionless interface level $\mathrm{h} / \mathrm{h}_{\mathrm{b}}$ as a function of the branch quality $\mathrm{x}_{3}$. 


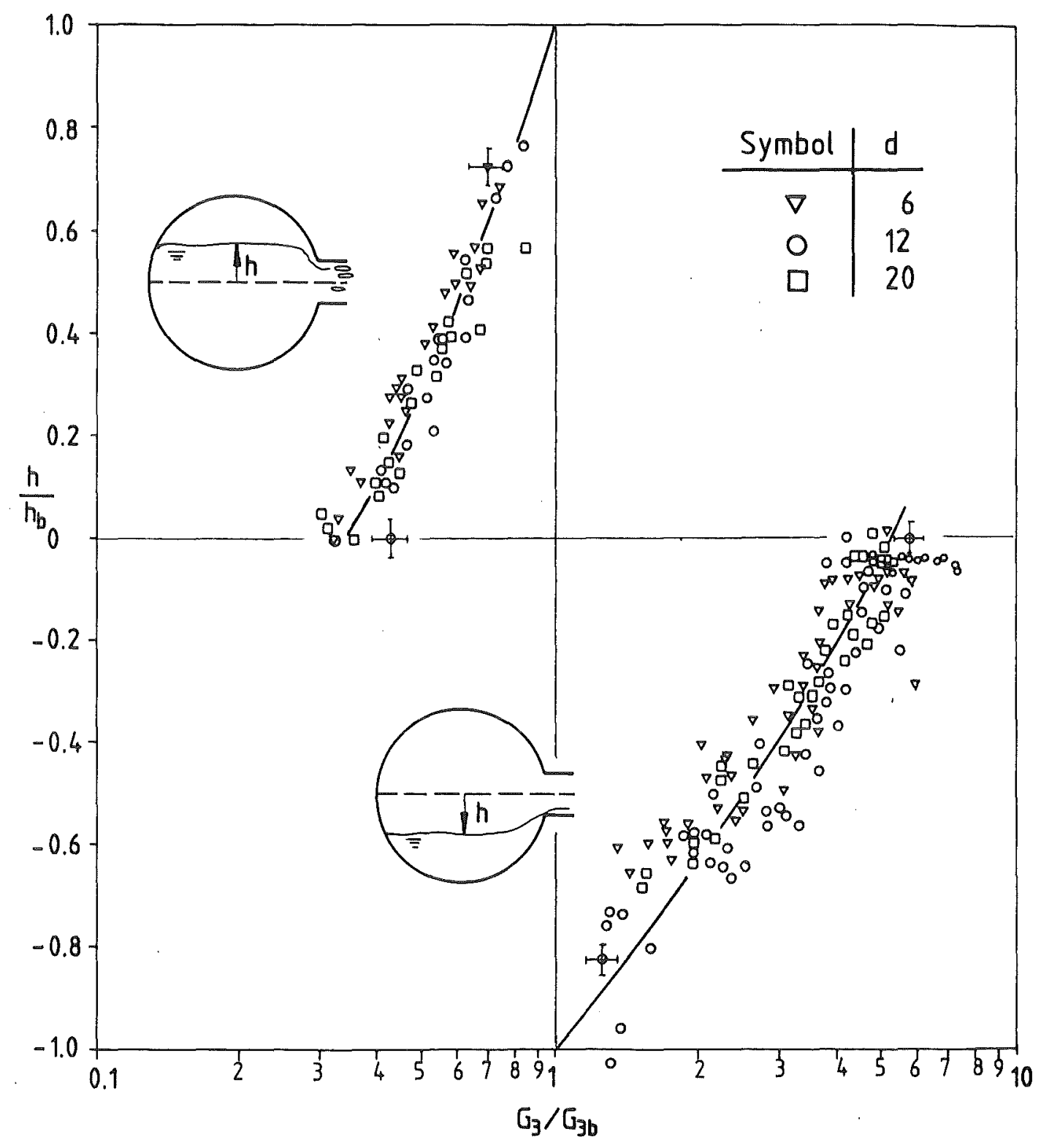

Fig. 37: Gas and liquid entrainment in horizontal branches: dimensionless interface level $\mathrm{h} / \mathrm{h}_{\mathrm{b}}$ as a function of the normalized branch mass $\operatorname{flux} G_{3} / G_{3 b}$. 


\subsubsection{Correlations for the Branch Quality \\ 6.2.3.1 Horizonta1 Branch}

The data for both liquid and gas entrainment were fitted with the following relationship:

$$
x_{3}=x_{0}^{\left(1+C \frac{h_{16}}{h_{6}}\right)}\left[1-\frac{1}{2} \frac{h_{h_{6}}}{h_{6}}\left(1+\frac{h_{1}}{h_{6}}\right) x_{0}^{\left(1-\frac{h^{2}}{h_{6}}\right)}\right]^{0.5}
$$

where $x_{0}$ represents the quality for $h=0$. To obtain this correlation, the linear dependency of $\mathrm{h} / \mathrm{h}_{\mathrm{b}}$ on $\log \mathrm{x}_{3}$ suggested by the results in Fig. 36 was first considered. Next, the term in brackets was introduced to give a quality tending to zero for $\mathrm{h} / \mathrm{h}_{\mathrm{b}}$ approaching unity. The coefficient $\mathrm{C}$ accounts for the change in the parameter $h_{b}$ when passing from liquid to gas entrainment (see Eq. (41)). Thus, for 1.e. $\left(h / h_{b} \leq 0\right), c=1$ and for g.e. $\left(h / h_{b} \geq 0\right), c=$

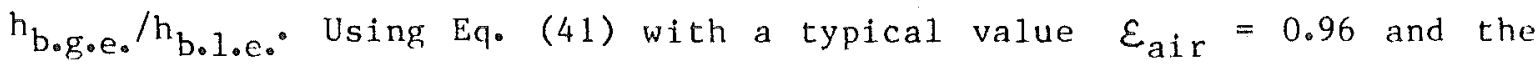
values of $\mathrm{K}$ given in Table 3 , the resulting coefficient for g.e. is $\mathrm{C}=1.09$.

The value of $x_{0}$ in Eq. (42) depends on the fluid densities; thus to obtain a general correlation, $x_{0}$ must be expressed as a function of these variables. A simple model for $x_{0}$ was developed assuming the conditions indicated in Fig. 38: the main pipe is filled with water up to the branch axis $(h=0) ; u_{g}$ and $\mathrm{u}_{1}$ represent the phase velocity of gas and liquid, respectively, at the branch entrance.

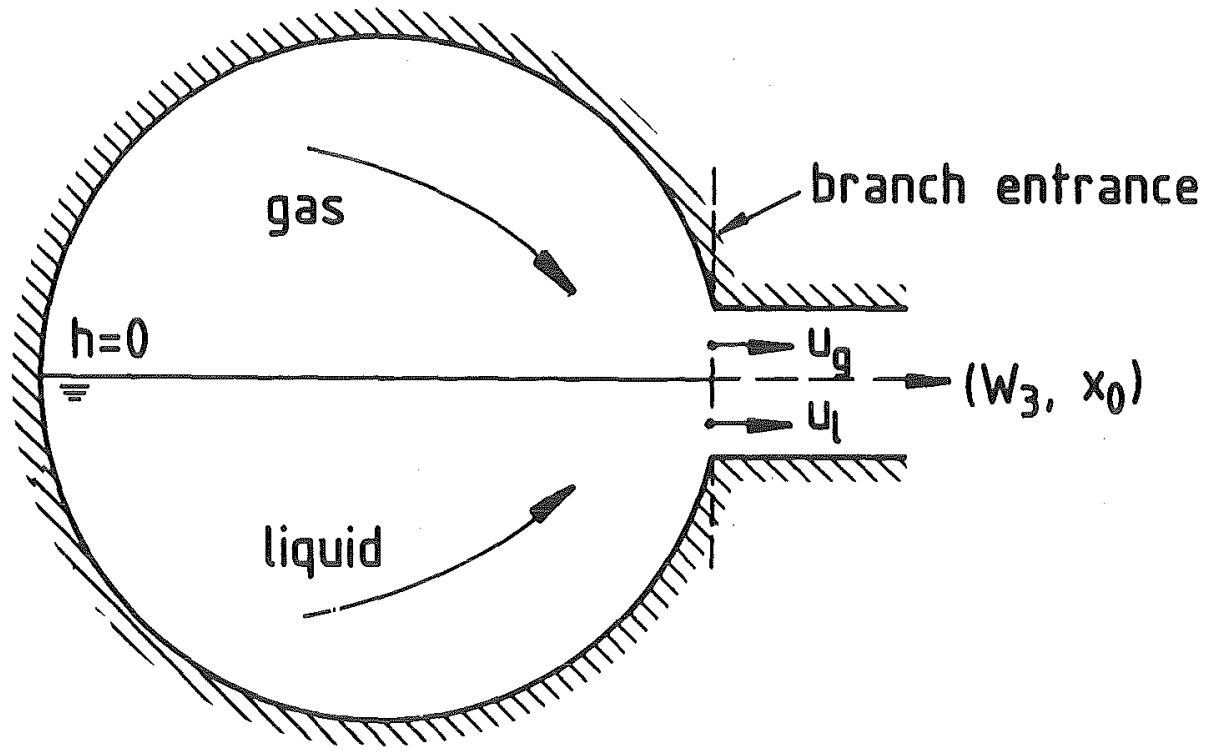

Fig. 38: Branch entrance conditions for interface level $\mathrm{h}=0$. 
With the hypothesis that for $\mathrm{h}=0$ both phases have

a) the same acceleration pressure drop,

b) the same cross sectional area $(\alpha=0.5)$,

the conditions at the branch entrance are given by:

$$
\Delta P_{\text {acc }}=\frac{1}{2} \rho_{g} \mu_{g}^{2}=\frac{1}{2} P_{e} \mu_{e}^{2}
$$

with $\alpha=0.5$, the phases satisfy: $\alpha \rho_{g} u_{g}=G_{3 g}=W_{3 g} / A_{3}$ and $\rho_{1} u_{1}=G_{31}=$ $W_{31} / A_{3}$. Therefore, Eq. (43) can be rearranged to give:

$$
\frac{\omega_{3 p}}{\omega_{3 g}}=\sqrt{\frac{\rho_{e}}{\rho_{g}}}
$$

which, combined with the definition $x_{3}=\left(1+w_{31} / w_{3 g}\right)^{-1}$, gives:

$$
x_{0}=\frac{1}{1+\sqrt{l_{e} / P_{g}}}
$$

Specific experiments performed to measure $x_{0}$ yielded, independently of $P_{1}$, $\Delta \mathrm{P}_{1-34}$ and $\mathrm{d}$, branch qualities about $15 \%$ higher than the values calculated with Eq. (45). This discrepancy is not surprising if the complexity of the flow processes generated in the vicinity of the branch entrance is considered. Thus, the final correlation proposed to predict the quality $\left(\mathrm{x}_{3, \mathrm{pr}}\right)$ in horizontal branches is:

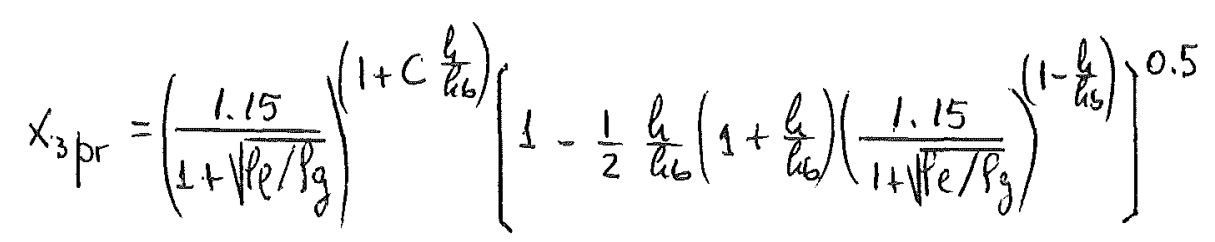

where:

$$
C=\begin{array}{ll}
1 \text { for l.e. } & \left(h / h_{6} \leq 0\right) \\
1.09 \text { for g.e } & \left(h / h_{6}>0\right)
\end{array}
$$

Figure 39 shows the ratio of measured to predicted quality $\left(\mathrm{x}_{3} / \mathrm{x}_{3 \mathrm{pr}}\right)$ as a function of $\mathrm{P}_{1}$. Data scatter around the value of one and no remarkable dependency on $P_{1}$ is observed. Figure 40 contains the same ratio as a function of $\mathrm{h} / \mathrm{h}_{\mathrm{b}}$. Again a fairly symmetric distribution was obtained both for gas and liquid entrainment. The standard deviation of the data is $\sigma=19 \%$ 


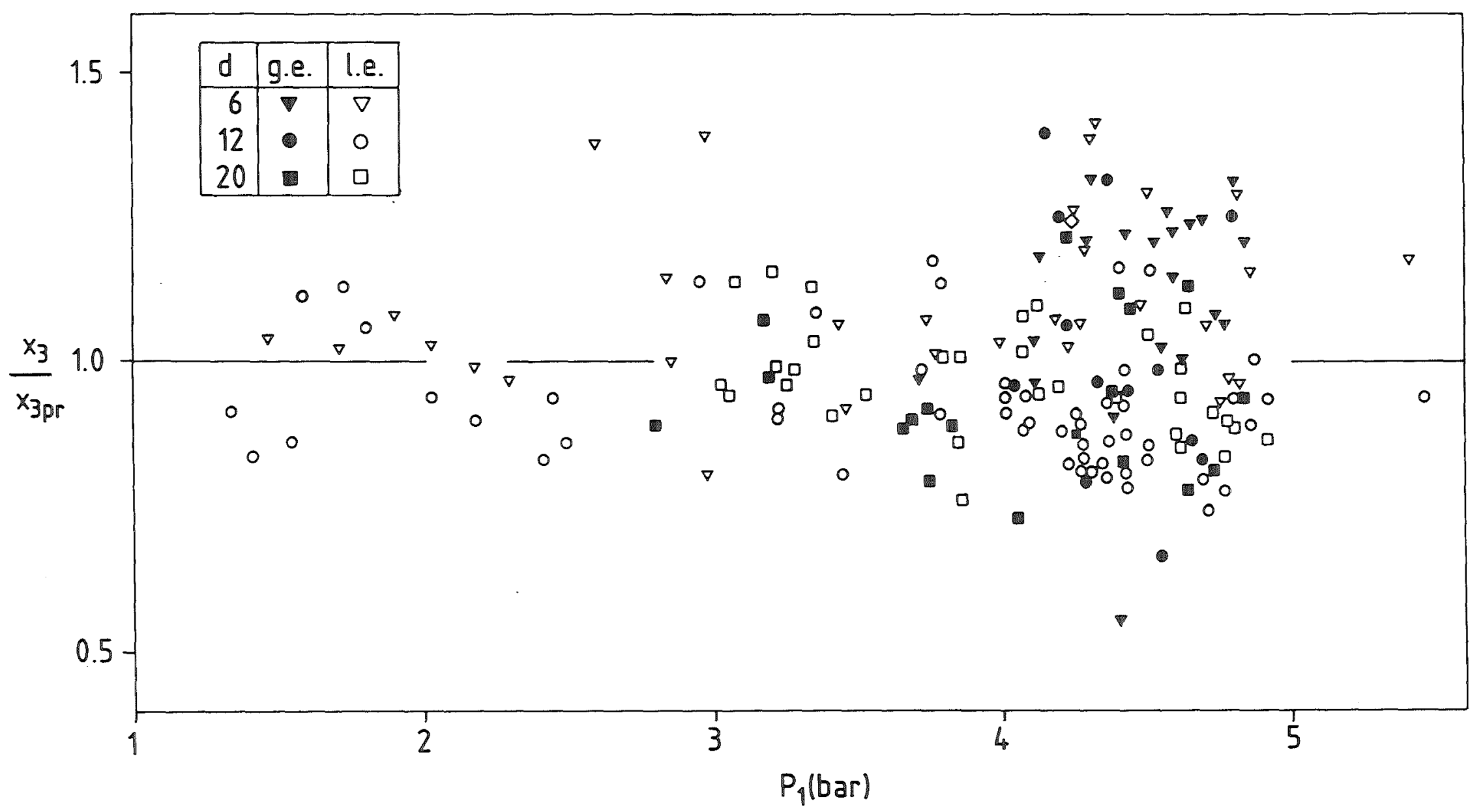

Fig. 39: Ratio of measured to predicted quality in horizontal branches as a function of system pressure $P_{1}$. 


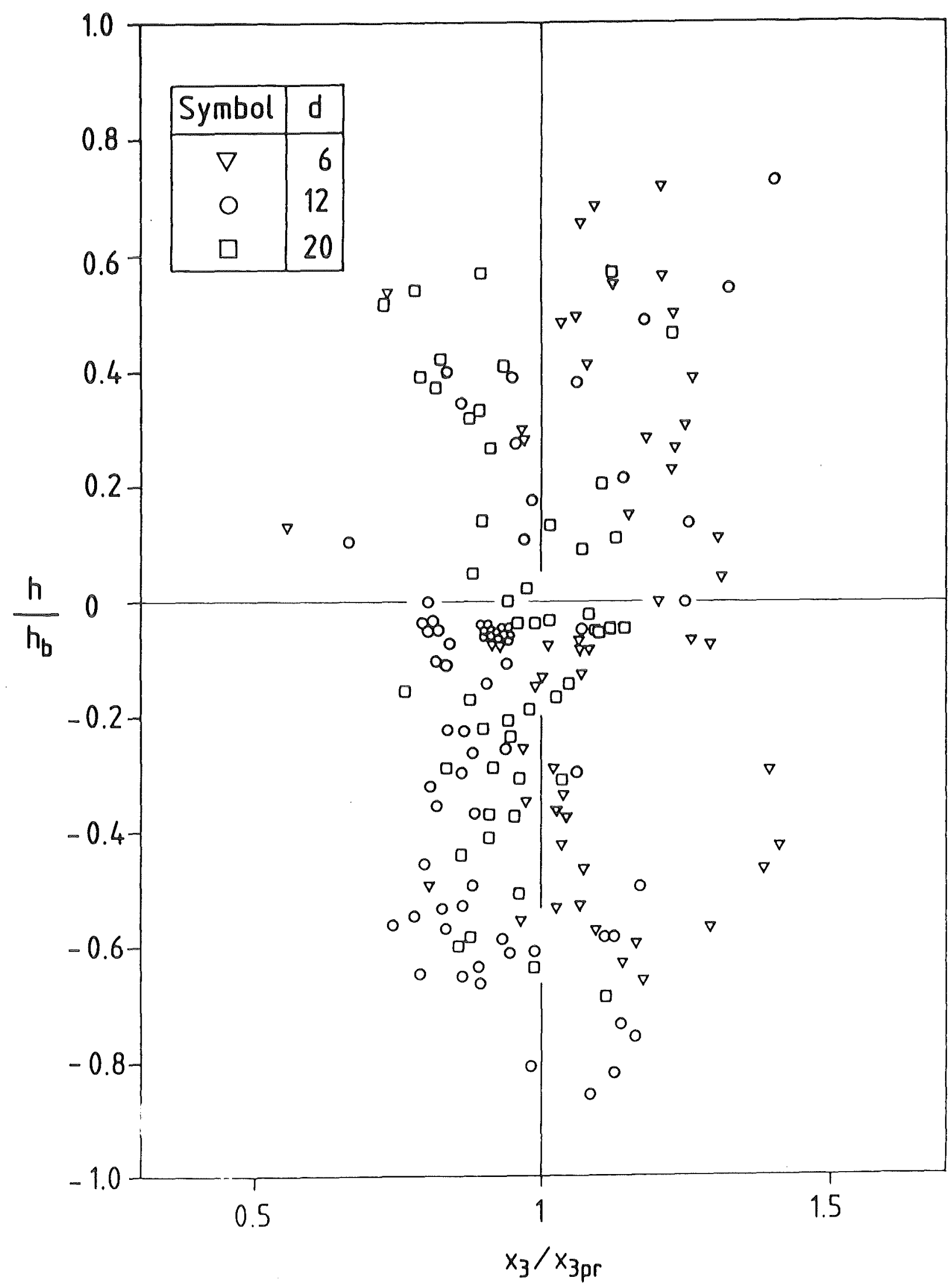

Fig. 40: Dimensionless interface level $\mathrm{h} / \mathrm{h}_{\mathrm{b}}$ as a function of measured to predicted quality in horizontal branches. 


\subsubsection{Donward Branches}

For downward branches $h$ was measured from the bottom of the horizontal pipe, which gives a quality $\mathrm{x}_{3}$ approaching unity for $\mathrm{h}$ approaching zero. The correlation (46) was modified to satisfy this condition, and the data in Fig. (33) were fitted with the following expression:

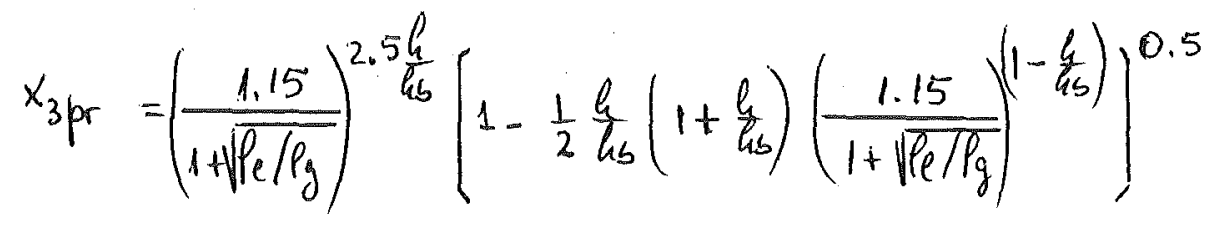

where the exponent 2.5 was adjusted to give a minimum mean square error.

Figure 41 shows the ratio $x_{3} / x_{3 p r}$ as a function of $h / h_{b}$ for g.e. in downvard branches. The strong data scatter observed at $h / h_{b}>0.9$ where the relative error in the measured quality is lower than $20 \%$, is due to the practically horizontal fitting curve at low qualities (see Fig. 33). In fact, for $\mathrm{x}_{3}<$ $0.1 \%\left(h / h_{b}>0.9\right)$ an error of $\pm 5 \%$ in $h / h_{b}$ can introduce an error greater than $100 \%$ in the predicted quality. However, in general, a symmetrical distribution of data around $x_{3} / x_{3 p r}=1$ is obtained. For $h / h_{b}<0.9$ the standard deviation is $\sigma=30 \%$

\section{$6.2 \cdot 3.3$ Upward Branch}

The 1.e. in upward branches can be considered as a flow phenomenon similar to the g.e. in downward branches with gas and liquid replaced by liquid and gas, respectively. Therefore, to predict the quality in upward branches an expression of the form:

$$
\mathrm{x}_{3 p r(\text { upward })}=1-\mathrm{x}_{3 \mathrm{pr}}(\text { downward })
$$

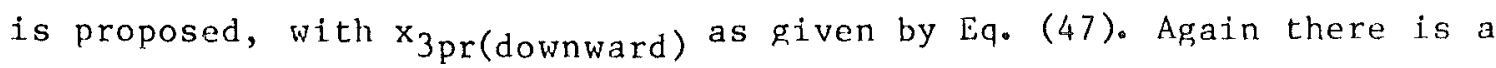
change in the normalization factor $h_{b}$ when passing from g.e. (downward) to 1.e. (upward), therefore the exponent $2.5 \mathrm{~h} / \mathrm{h}_{\mathrm{b}}$ used in Eq. (47) was replaced

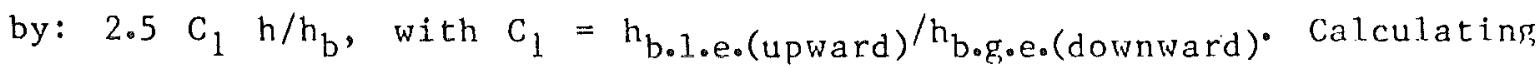
this coefficient as previously (Section 6.2 .3 .1 ) results in: $C_{1}=0.8$, and the expression proposed in (48) becomes: 


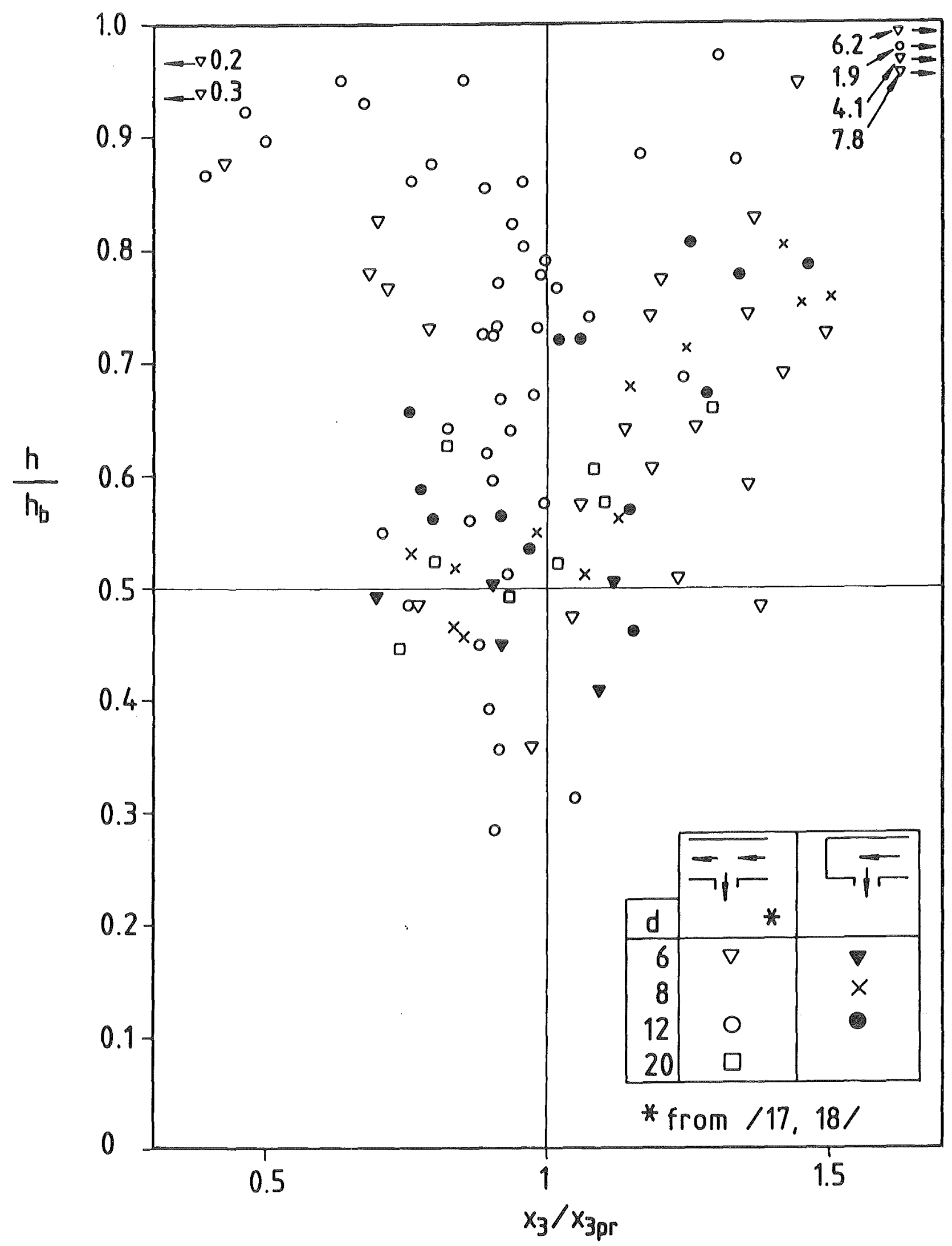

Fig. 41: Dimensionless interface level $\mathrm{h} / \mathrm{h}_{\mathrm{b}}$ as a Eunction of measured to predicted quality in downward branches. 


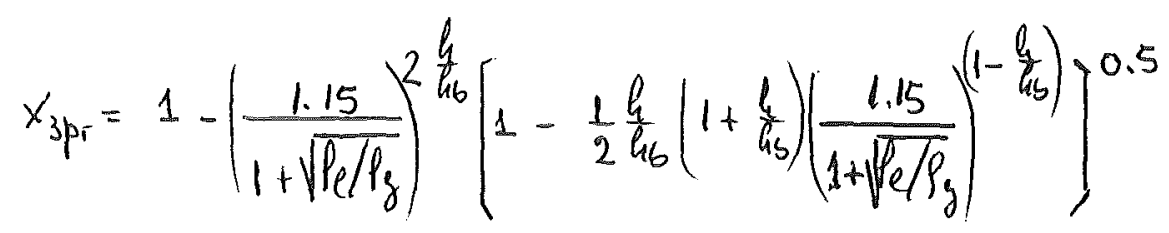

This correlation was used to draw the dashed curve in Fig. 35a assuming $\mathrm{P}_{1}=4$ bar. The discrepancy with respect to the mean value of the experimental results is not greater than $1 \%$

The ratio $x_{3} / x_{3 p r}$ as a function of $h / h_{b}$ is presented in Fig. 42. An increasing scatter around the value $x_{3} / x_{3 p r}=1$ is observed for decreasing $h / h_{b}$ which coincides with the occurrence of waves and intermittent slug in the main pipe. Discarding the points corresponding to developed slug flow, the standard deviation of the data is very low: $\sigma=1.5 \%$

The results in Section 6.2 .3 give correlations to predict the branch quality which are functions of the densities ratio and dimensionless interface level only:

$$
x_{3 p r}=f\left(\frac{\rho_{l}}{\rho_{g}}, \frac{h_{h}}{h_{s}}\right)
$$

These correlations are considered as applicable to arbitrary fluids and arbitrary flow geometries downstream of the branch entrance, that means, they should be valid for orifices, pipe studs, nozzles, etc. 


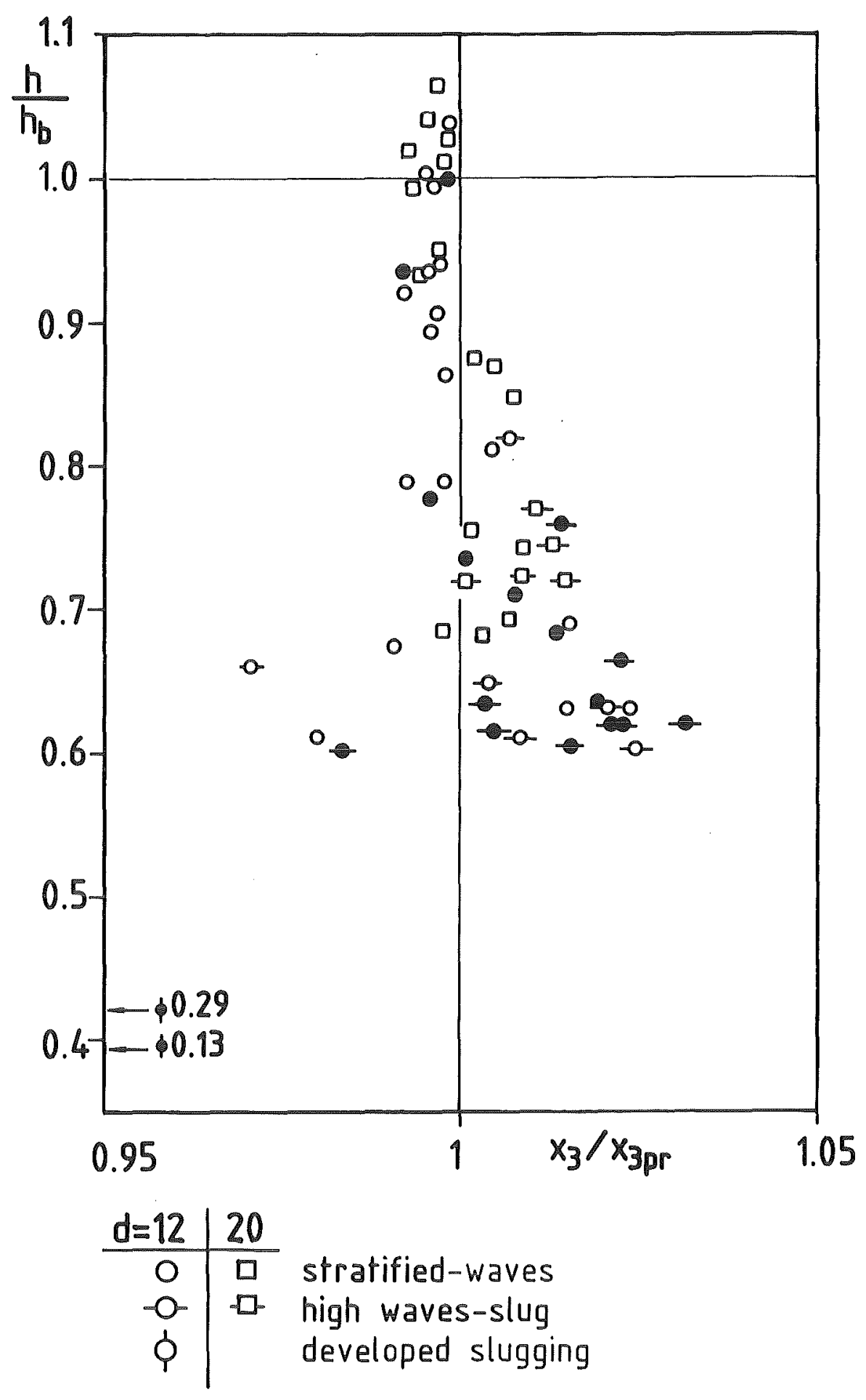

full symbols: critical flow

Fig. 42: Dimensionless interface level $\mathrm{h} / \mathrm{h}$ as a function of measured to predicted quality in upward branches. 


\subsubsection{Correlations for the Branch Mass Flux}

For two-phase flow the expansion coefficient $\varepsilon$ in Eq. (37) was replaced by the expression $[1+\alpha(\varepsilon-1)]$, where $\alpha$ is the void fraction, therefore:

$$
G_{3}=?[1+\alpha(\varepsilon-1)] \sqrt{\rho \Delta P_{1-34}}
$$

With an appropiate model giving $\alpha$ and $\rho$ as a function of $x_{3}$, Eq. (51) could be combined with the results in Section 6.2 .3 to predict the total branch mass flux. llodels for the branch mass flux are strongly dependent on the branch geometry and, therefore, will be different for orifices, nozzles, pipe studs etc.

It was not the aim of this investigation to model in detail the branch mass flux for the special geometry used in the present experiments therefore only the simplest model, the homogeneous model, has been applied and Eq. (51) is rewritten as follows:

$$
\left.G_{3 H}=\right\}\left[1+\alpha_{H}(\varepsilon-1)\right] \sqrt{\rho_{H} \Delta P_{1-34}}
$$

where

$$
\alpha_{H}=\left(1+\frac{1-x_{3}}{x l_{l} / \rho_{g}}\right)^{-1} ; \quad \varphi_{H}=\left(\frac{x_{3}}{\rho_{g}}+\frac{1-x_{3}}{\rho_{c}}\right)^{-1}
$$

For each branch diameter the ratio of measured to homogeneous mass flux, $\mathrm{G}_{3} / \mathrm{G}_{3 \mathrm{H}}$, was plotted as a function of the quality measured in experiments with the three branch orientations.

Results for each branch diameter are presented in Fig. 43. It is obvious that the homogeneous model does not describe properly the branch mass flux for qualities between 2 and $60 \%$. However, almost the same dependency of $G_{3} / G_{3 H}$ on $\mathrm{X}_{3}$ was obtained for the different diameters and, therefore, it was possible to use a single fitting function $F\left(x_{3}\right)$, valid for the branches used and expressed by:

$$
\frac{G_{3}}{G_{3 H}} \simeq F\left(x_{3}\right)= \begin{cases}1+\left(1-x_{3}\right)\left(-61 x_{3}^{2}+12.2 x_{3}\right) & ; x_{3} \leqslant 0.1 \\ 1+\left(1-x_{3}\right) x_{3}^{0.12} e^{-2.2 x} & ; x_{3} \geqslant 0.1\end{cases}
$$



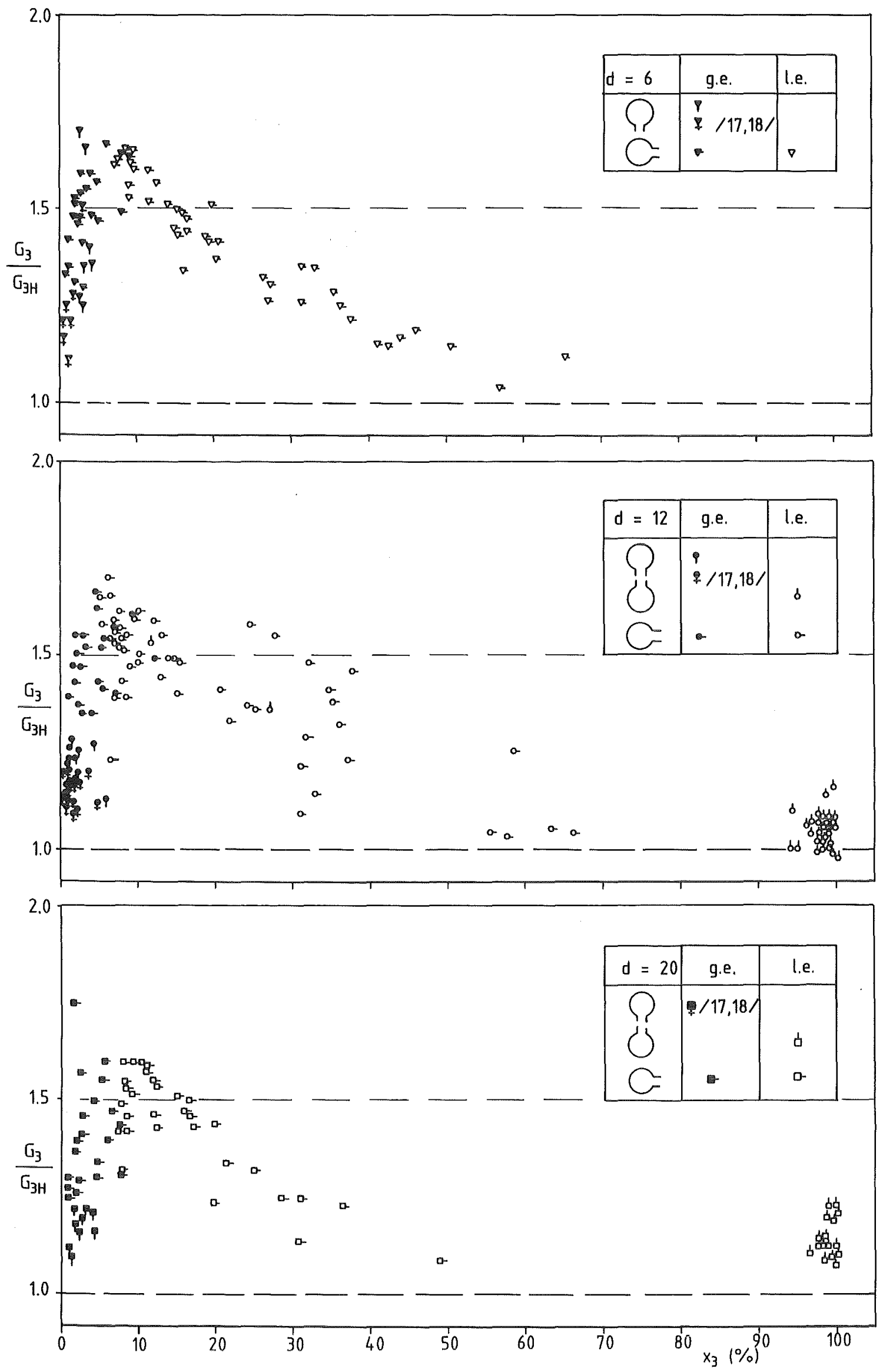

Fig. 43: Ratio of measured to homogeneous branch mass flux $\mathrm{C}_{3} / \mathrm{C}_{3}$ as a function of the branch quality $x_{3}$. 
It is to note that this function should not be used for other branch geometries. To check the validity of Eq. (53) for the geometries under consideration, a predicted branch mass flux was calculated as a function of the measured quality $\mathrm{x}_{3}$ as follows:

$$
\mathrm{G}_{3 \mathrm{Pr}_{\mathrm{r}}}\left(\mathrm{x}_{3}\right)=\mathrm{G}_{3 \mathrm{H}}\left(\mathrm{x}_{3}\right) \mathrm{F}\left(\mathrm{x}_{3}\right)
$$

With this procedure the ratios of measured to predicted branch mass fluxes $\mathrm{G}_{3} / \mathrm{G}_{3 p r}$ were calculated for each branch orientation and plotted as a funtion of: $x_{3}$, as shown in Fig. 44. Figure 44a, corresponding to 1.e. in upward branches, shows that for $\mathrm{x}_{3}>97 \%$ the measured $\mathrm{G}_{3}$ is about $8 \%$ greater than the predicted one. A discrepancy of the same order is observed for $x_{3}<1 \%$ in Fig. 44b, corresponding to g.e. in downward branches, whereas in Fig. 44c, for gas and liquid entrainment in horizontal branches, a fairly symmetric distribution around the value one, is observed for all qualities.

The differences between the measured and predicted mass fluxes can be corrected by fitting the data in Fig. 43 with a different function $F\left(x_{3}\right)$ for each branch diameter, which gives a different $G_{3 p r}$ for each branch; but again this was not the aim of this investigation. Therefore, the final correlation proposed to predict the branch mass flux is:

$$
G_{3 p r}=G_{3 H}\left(x_{3 p r}\right) F\left(x_{3 p r}\right)
$$

where $x_{3 p r}$ is calculated with the correlations obtained in Section 6.2.3 for each branch orientation.

The corresponding results, showing the ratio $G_{3} / G_{3 p r}$ for the diffeent branch orientations as a function of $h / h_{b}$, are presented in Figs. 45-47. The standard deviation of data from 1 is $\sigma=18 \%$ for upward branches (Fig. 45), $\sigma=12 \%$ for downward branches (Fig. 46), and $\sigma=9.5 \%$ for horizontal branches (Fig. 47). These relative small deviations correlate with the asymmetries observed in Fig. 44 . 

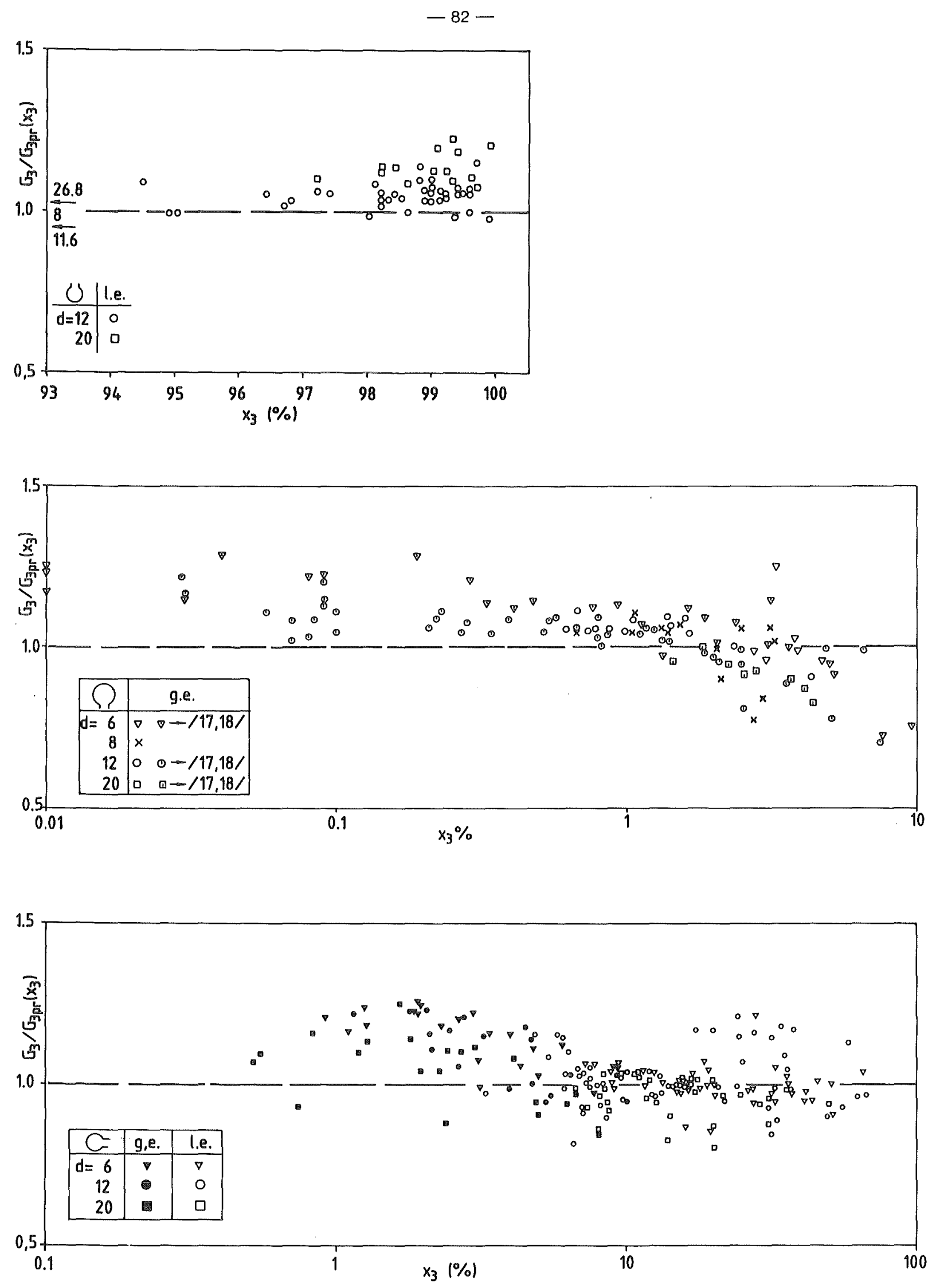

Fig. 44: Measured to predicted mass $f 1 u x G_{3} / G_{3 p r}\left(x_{3}\right)$ as a function of the quality $\mathrm{x}_{3}$ in upward, downward and horizontal branches. 


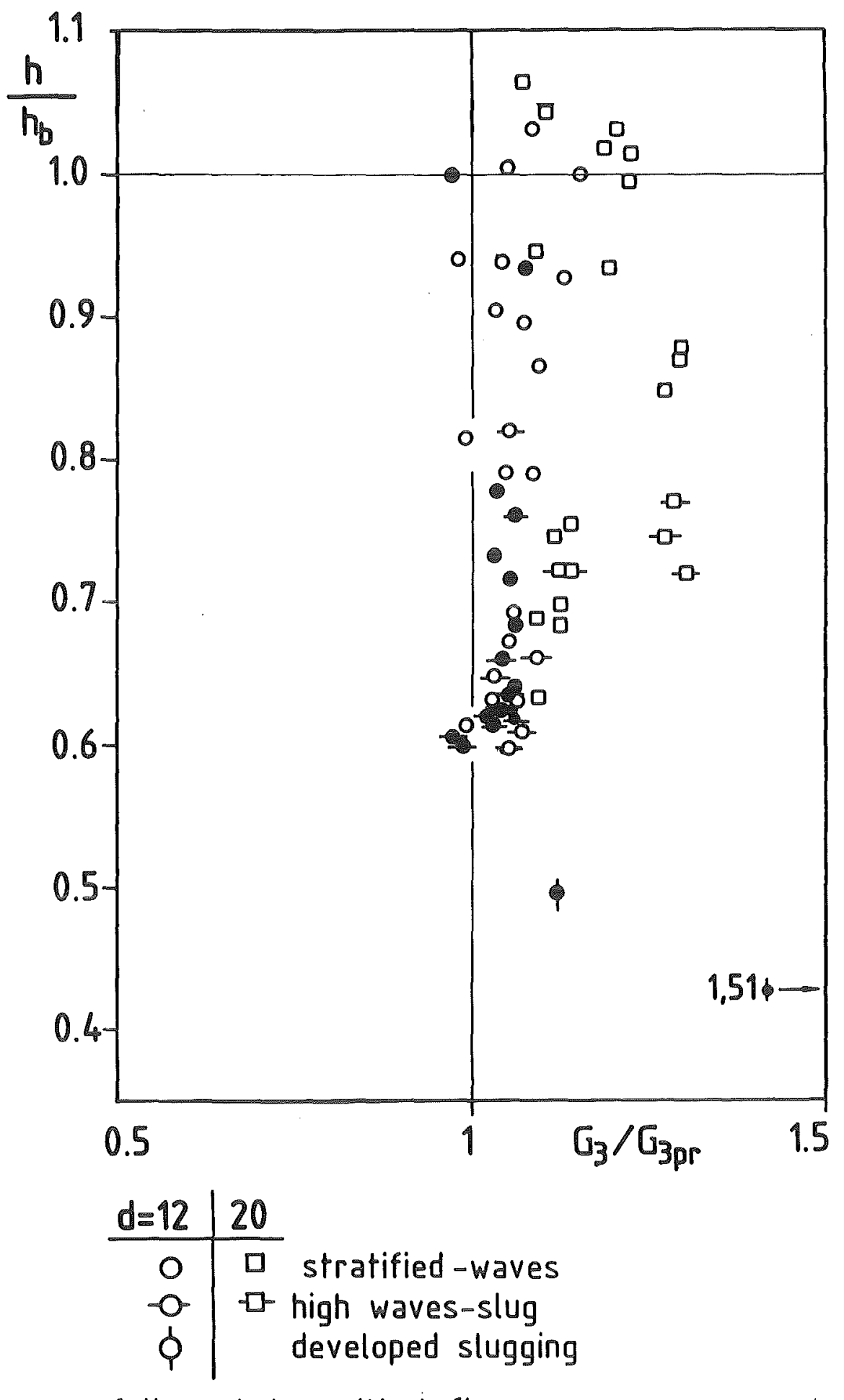

full symbols: critical flow

Fig. 45: Dimensionless interface leve $1 \mathrm{~h} / \mathrm{h}_{\mathrm{b}}$ as a function of measured to predicted mass flux $G_{3} / G_{3 p r}$ in upward branches. 


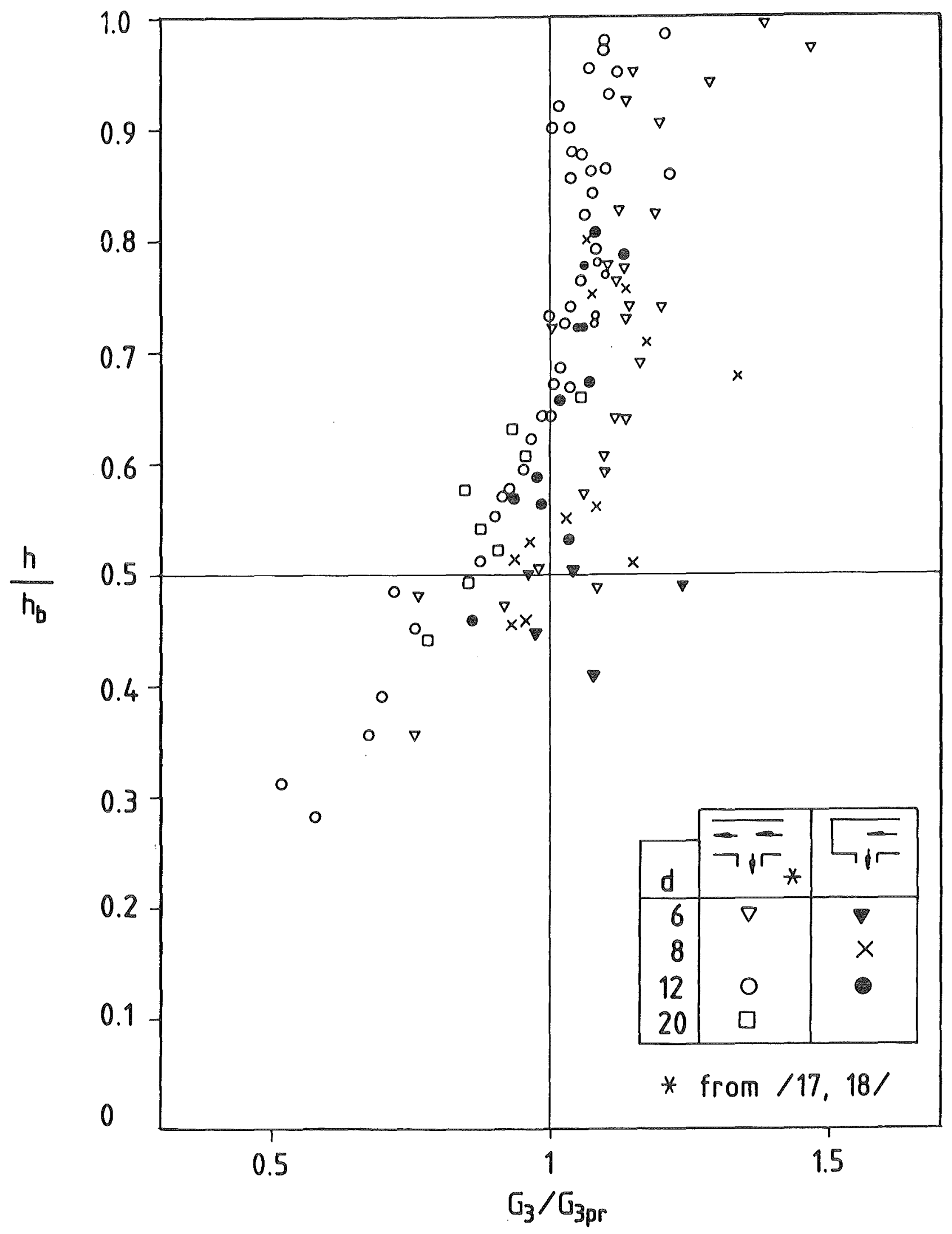

Fig. 46: Dimensionless interface level $\mathrm{h} / \mathrm{h}_{\mathrm{b}}$ as a function of measured to predicted mass flux $G_{3} / G_{3 p r}$ in downward branches. 


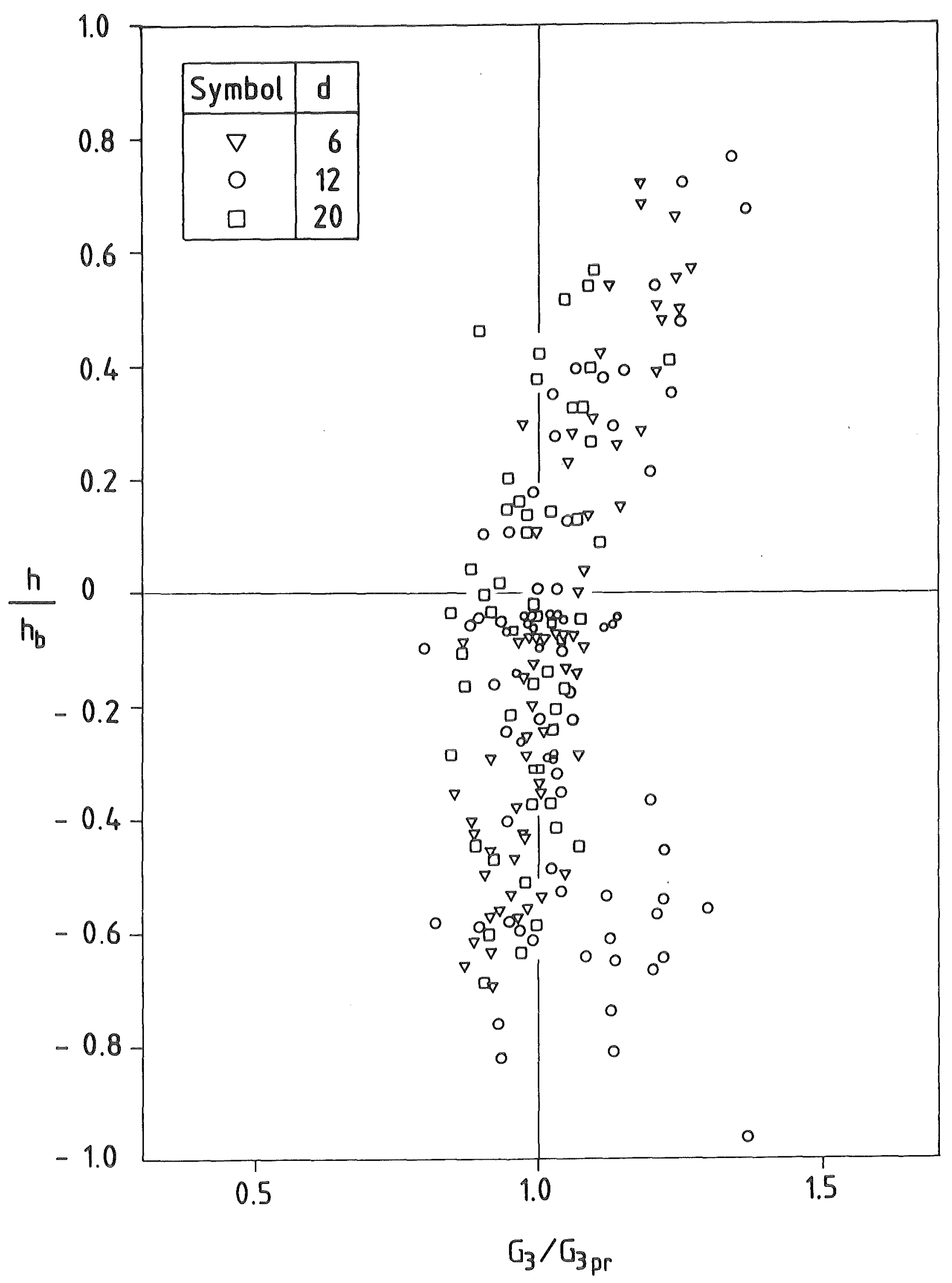

Fig. 47: Dimensionless interface level $\mathrm{h} / \mathrm{h}_{\mathrm{b}}$ as a function of measured to predicted mass flux $G_{3} / G_{3 p r}$ in horizontal branches. 


\section{Critical Branch Mass-F1ux}

\subsection{The Homogeneous Equilibrium Model (HEM)}

The model discussed up to now is valid for subcritical gas-liquid flow. However in a small break LOCA critical steam-water flow at high pressure occurs; therefore, in the previous correlations an appropiate model for critical twophase flow has to be used. Such a model will depend on the break geometry and many articles have been published on this subject (compare e.g. D'Auria and Vigni, /34/). It is not the aim of this work to model in detail the critical mass flux for the special break geometry used in the present experiments but rather to explain the procedure and show typical tendencies.

For the following derivations it is assumed that during the small break LOCA:

- the interface is ideally stratified;

- there is no effect of the flashing, produced by the pressure drop in the vicinity of the break on the flow field near the horizontal interface;

- there are no bubbles in the liquid produced by the decrease of the system pressure or by heat transfer from the pipe walls.

On these hypotheses the very simple Homogeneous Equilibrium Model (HE1), $135 /$, will be applied which gives satisfactory results for long pipes with a large ratio $1 / \mathrm{d}$ (compare Wallis $/ 36 /$ ).

The basic assumptions of the HEM (for details see Appendix A3) are:

- llomogeneity: both phase have the same velocity and their channel averaged properties give the thermodynamical properties of the mixture.

- Thermodynamical equilibrium: liquid and vapor have the same pressure and temperature.

- Isentropic flow: neither heat nor work are interchanged with the exterior.

The HEM provides a thermodynamic connection of the flow properties upstream of the break and in the break cross section. Weigand et a1. $/ 37 /$, for instance, have used the HEM to develop a computational model for analyzing trophase jets, and they give critical flow charts relating the break mass flux and thermodynamic properties to the upstream stagnation conditions. In particular, Figure 48 (from /37/) gives the HEM critical mass-flux in the break cross section as a function of the stagnation quality and stagnation tempera- 

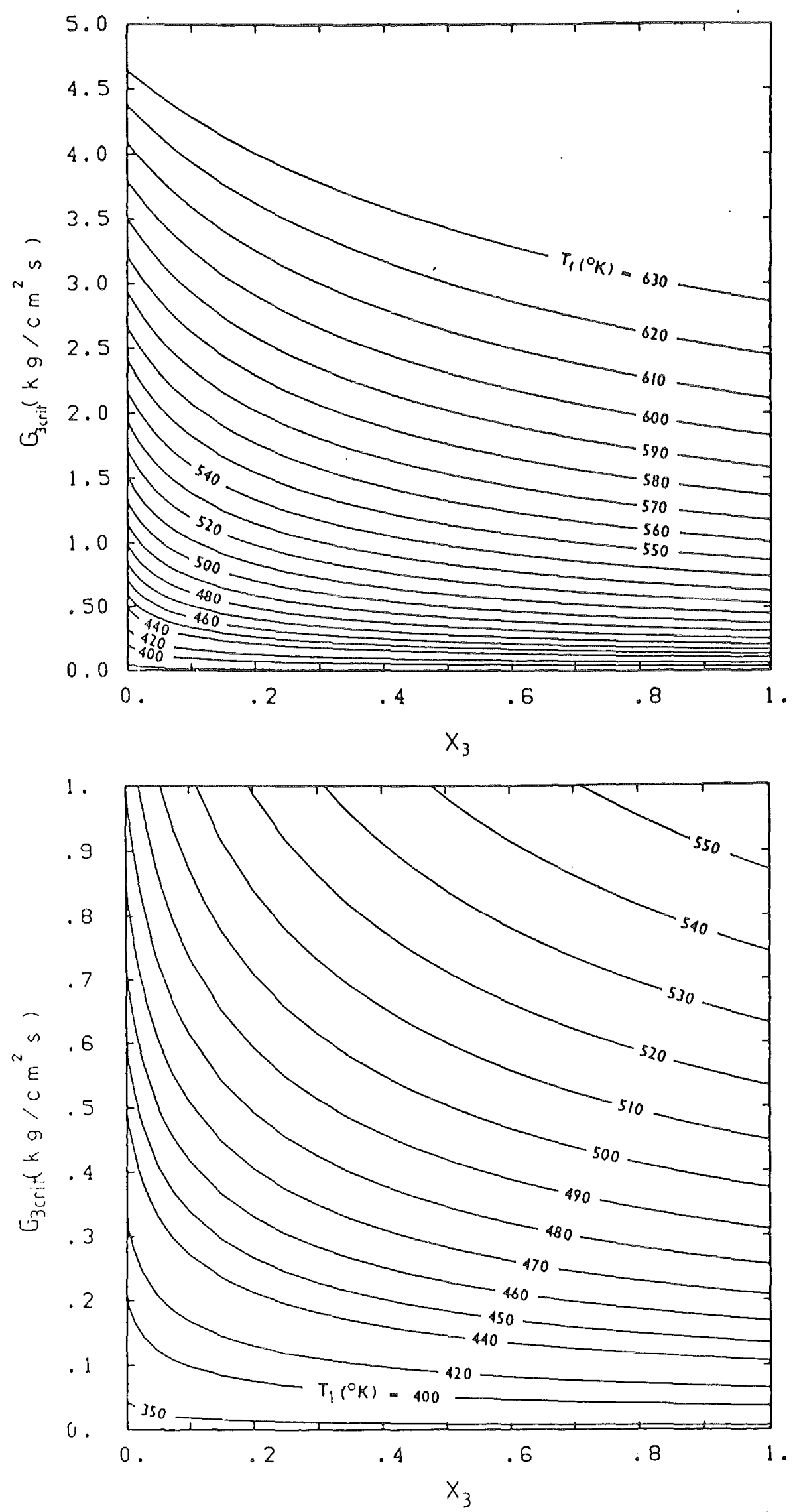

Fig. 48: HEM critical mass flux as a function of stagnation quality and temperature. 
ture of a saturated mixture. The information provided by these curves will be used in the next section to predict the critical mass flux in horizontal branches.

\subsection{Quality and Critical Mass Flux in Horizontal Branches}

The model proposed for $x_{0}$ in Section 6.2 .3 .1 is assumed to be independent of the flow behavior downstream of the branch entrance; therefore, it can be applied also to critical branch flows. However, the normalization factor $h_{b}$ has to be calculated with the critical value of $N_{3 b}$ given by the HEM curve corresponding to the system pressure.

For constant $P_{1}$ the correlation (5) gives:

$$
\begin{aligned}
& h_{b . g, e .}=\frac{k_{g e} w_{3 l c r i t}^{0.4}}{\left[g \rho_{l}\left(\rho_{e}-\rho_{g}\right)\right]^{0.2}} \\
& h_{b . l_{e}}=\frac{k_{l e} \omega_{3 g c r i t}^{0.4}}{\left[g \rho_{g}\left(\rho_{e}-\rho_{g}\right)\right]^{0.2}}
\end{aligned}
$$

Equations (56) and (57) were used to calculate $h_{b}$ for arbitrary values of $h>$ 0 and $\mathrm{h}<0$, respectively. The correlation (46) was used to predict the corresponding quality.

With this value of $x_{3}$ interpreted as the stagnation quality again the HEM curve for $P_{1}$ was used to determine the corresponding critical branch mass f. lux。

\subsection{Application of the Model}

The procedure presented above was applied to predict the branch quality and mass-flux, in case of a LOCA produced by a side break in a horizontal coolant pipe of a standard reactor (I.D. $750 \mathrm{~mm}$ ). The branch diameter was assumed to be $49 \mathrm{~mm}$ to simulate a break with a ratio of break to main pipe cross sectional area of $0.4 \%$ 
Calculations based on system pressures of 5 and $10 \mathrm{MPa}$ were made taking into account the values in the table below:

\begin{tabular}{|c|c|c|c|}
\hline Parameters & $\mathrm{P}_{1}=5 \mathrm{MPa}$ & $P_{1}=10 \mathrm{MPa}$ & References \\
\hline Tsat $\quad o_{K}$ & 537 & 584.15 & steam-water \\
\hline Pg sat $\mathrm{kg} \mathrm{m}^{-3}$ & 25.40 & 55.5 & thermodynam. \\
\hline$\rho_{1}$ sat $\mathrm{kg} \mathrm{m}^{-3}$ & 778 & 688 & properties \\
\hline $\mathrm{G}_{3 \mathrm{~g}} \operatorname{crit} 10^{-4} \mathrm{~kg} \mathrm{~m}^{-3} \mathrm{~s}^{-1}$ & 0.714 & 1.45 & HEM (Fig. 48) \\
\hline $\mathrm{G}_{31 \mathrm{crit}} 10^{-4} \mathrm{~kg} \mathrm{~m}^{-3} \mathrm{~s}^{-1}$ & 2.067 & 3.35 & HEM (Fig. 48) \\
\hline$h_{\text {bge }} \quad m$ & 0.144 & 0.185 & $\mathrm{Eq} \cdot 56$ \\
\hline$h_{\text {ble }}$ & 0.172 & 0.202 & $\mathrm{Eq}_{\mathrm{q}} \cdot 57$ \\
\hline
\end{tabular}

Table 5: Parameters used to predict the quality in branches with critical mass flux.

The results were plotted as a function of $h_{1} / D$ (Fig. 49), where $h_{1}=D / 2+h$ is the liquid level measured from the bottom of the main pipe.

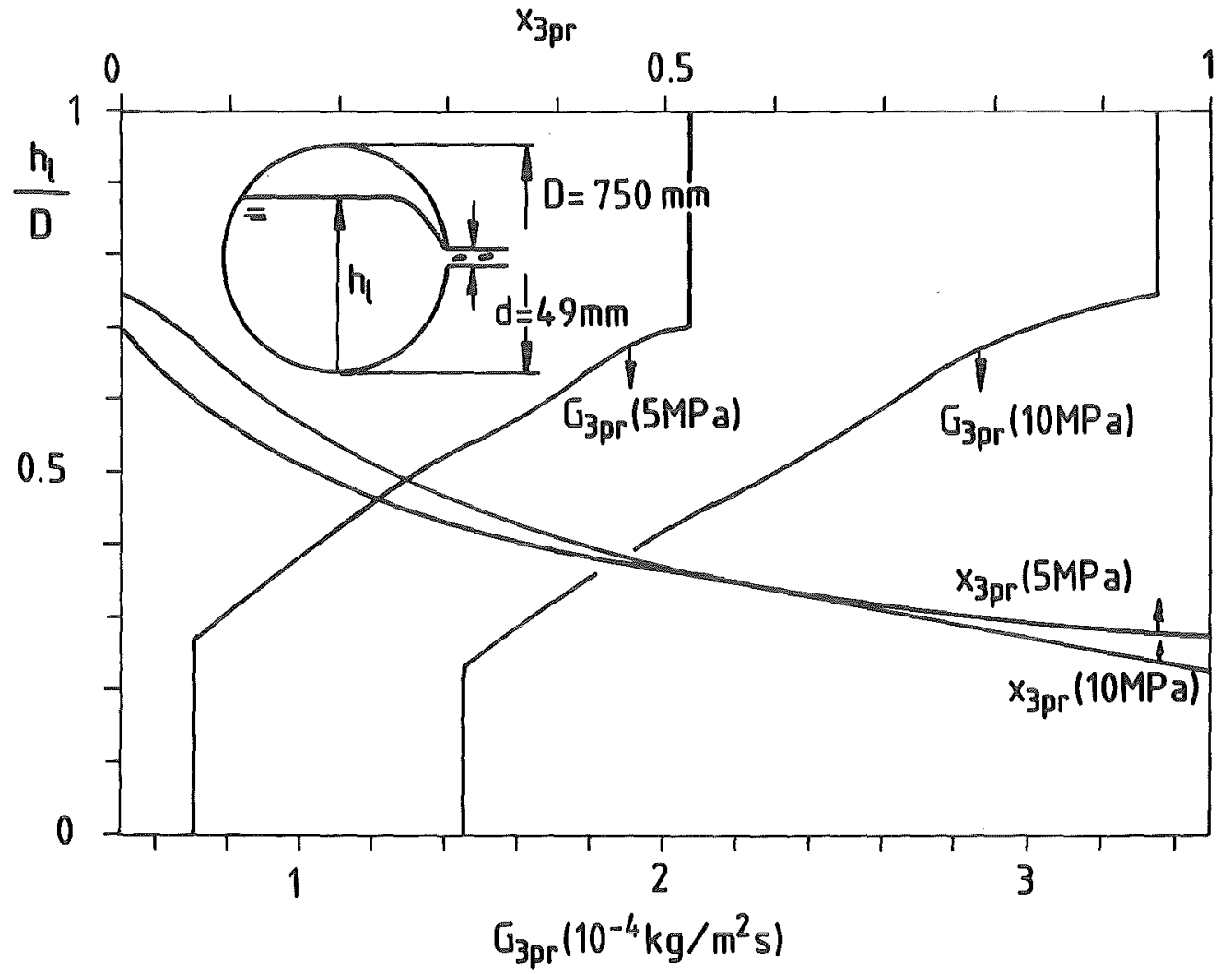

Fig. 49: Dependency of predicted branch quality $x_{3 p r}$ and critical mass flux $G_{3 p r}$ on the dimensionless liquid level $h_{1 / D}$. 
Starting at $h_{1} / D=1$ and for decreasing values of the liquid level. in the main pipe, the predicted branch quality and mass flux are constant until the point is reached at which the vapor filling the pipe portion above the interface, begins to be entrained in the branch. Below this point, the branch flow is described by $x_{3 p r}$ ranging from 0 to 1 and the corresponding $\mathrm{G}_{3 \mathrm{pr}}$ decreasing to its minimal value where no longer liquid entrainment occurs. For smaller values of $h_{1}$, only vapor flows through the branch, therefore, $x_{3 p r}=$ 1 and $G_{3 p r}=$ constant. As expected, a higher pressure extends the range of $h_{1} / D$ for which steam or water entrainment occurs.

\subsection{Comparison with other Models}

Two other models for the branch quality which will be combined with the HEM will be considered below:

a) the model used in the RELAP4 computer code /38/, originally developed for large break LOCA with the assumption made that the branch inlet void fraction $\alpha_{3}$ is equal to the upstream void fraction $\alpha_{1}$,:

$$
\alpha_{3}=\alpha_{1}
$$

b) the model used in the RELAP5 computer code /39/, which was specially developed to calculate the mass discharge from horizontal pipes with stratified flow through side breaks.

In RELAP5 (see details in Appendix A4) the limit gas velocity $v_{g L}$ in the horizontal pipe with diameter $\mathrm{D}$ and liquid level $\mathrm{h}_{1}$, is defined as:

$$
v_{g L}=\frac{1}{4 \sqrt{2}}(1-\cos \theta)\left[D g \frac{\rho_{e}-\rho_{g}}{\rho_{g}} \frac{2 \theta-\sin 2 \theta}{\sin \theta}\right]
$$

where $\theta=\cos ^{-1}\left(2 h_{1} / D-1\right)$. The void fraction $\alpha_{3}$, at the inlet of a horizontal branch of diameter $d$, is defined as follows: for the interface above and below the branch entrance, respectively:

$$
\begin{array}{ll}
\alpha_{3}=\alpha_{1}\left(\frac{v_{i g}}{v_{g L}}\right)^{0.5} & \text { for } \quad h_{\ell}>\frac{D+d}{2} \\
\alpha_{3}=1-\left(1-\alpha_{1}\right)\left(\frac{v_{1 g}}{v_{g L}}\right)^{0.5} \text { for } h_{\ell}<\frac{D-d}{2}
\end{array}
$$


When the liquid level lies within the outlet area, $i_{\bullet} e$, when $(D-d) / 2<h_{1}<$ $(d+D) / 2, \alpha_{3}$ is obtained by interpolation of the two void fractions computed at the boundaries.

To predict the branch quality, the correlation corresponding to homogeneous branch mass-flow was used:

$$
x_{3 p r}=\left[1+\left(\frac{1}{\alpha_{3 p r}}-1\right) \frac{\rho_{\rho}}{\rho_{g}}\right]
$$

where $\alpha_{3 p r}$ is the value given by RELAP4 (Eq. (58)) or RELAP5 using Eqs. (6061 ), with the value $v_{1 g}=1 \mathrm{~m} / \mathrm{s}$.

Results for branch quality and mass flux given by the different models are compared in Figures 50 and 51 .

Figure 50 shows that the discrepancy between the predicted qualities depends on the liquid level. For $h_{1} / D$ below the value corresponding to b.g.e., the new qualities are greater than those given by RELAP4 and RELAP5, the maximum difference representing one order of magnitude.

The corresponding values for $\mathrm{G}_{3 \mathrm{pr}}$ are compared in Figure 51 For a wide range of $h_{1} / D$ the mass fluxes calculated with the new model are smaller than the values given by RELAP4 and RELAP5.

The constant values of $\mathrm{x}_{3 \mathrm{pr}}$ and $\mathrm{G}_{3 \mathrm{pr}}$ above and below the levels corresponding, to b.g.e. and b.1.e., respectively, constitute a difference introduced by the model proposed in the present investigation. 

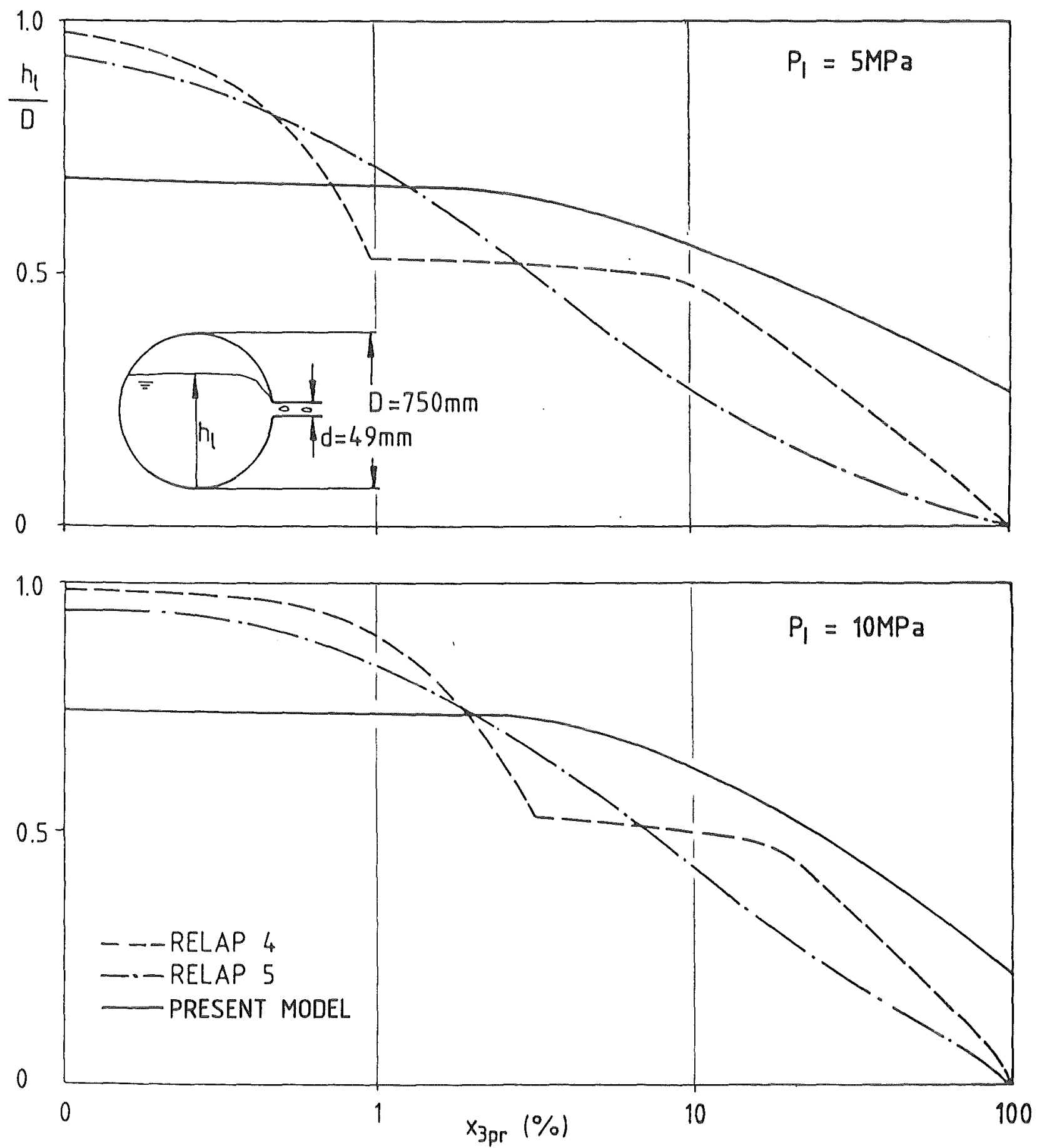

Fig. 50: Comparison of the different models to predict the quality for critical branch flow and varying liquid level in the main pipe. 

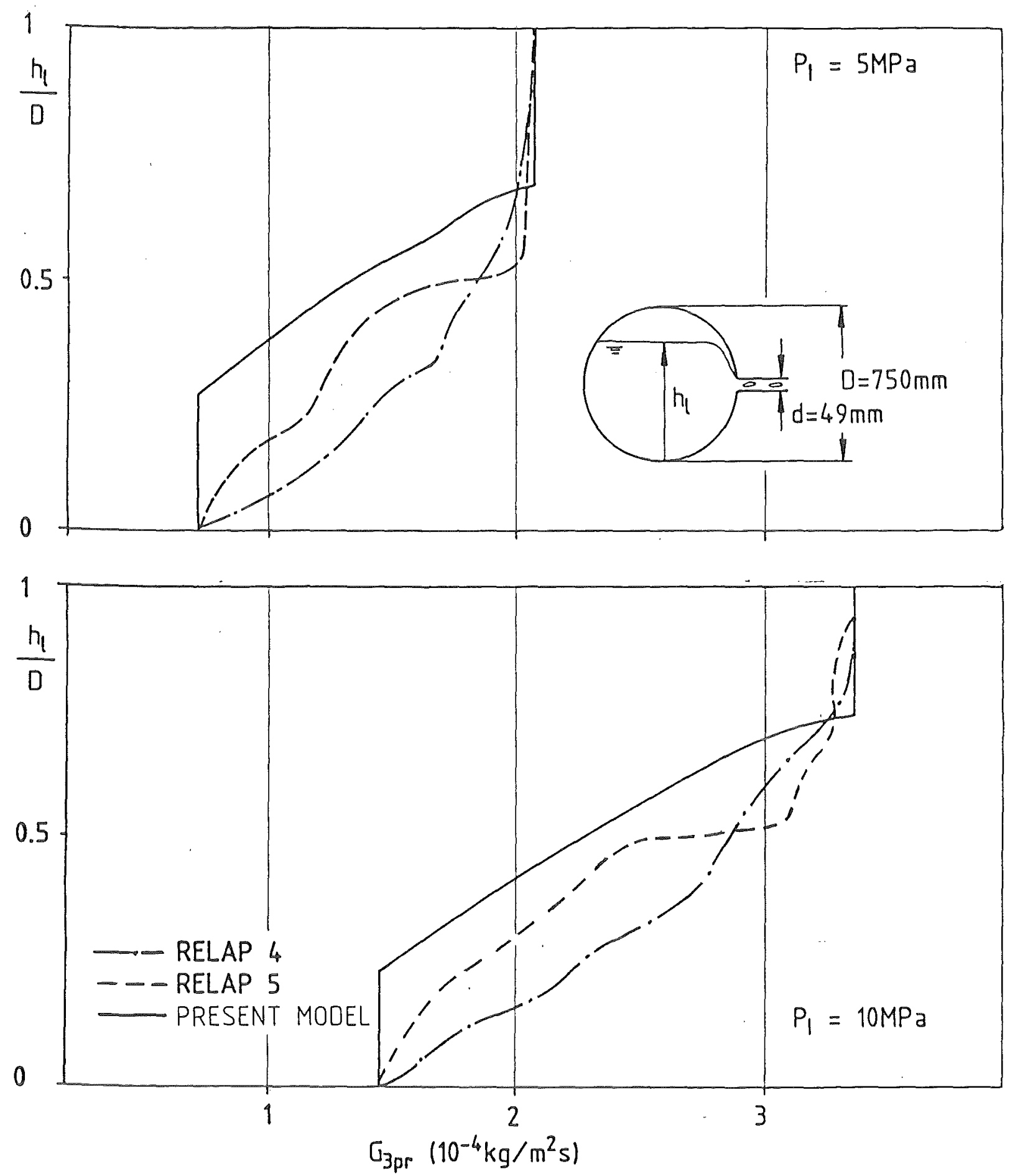

Fig. 51: Comparison of the different models to predict the critical branch mass flux for varying liquid levels in the main pipe. 
Summary

The mass flow rate and quality through small branches at the bottom, the top or the side of a horizontal pipe with stratified flow was investigated.

The problem is of importance for the study of loss of coolant accidents (LOCA) in nuclear reactors. Limited knowledge of this subject was available from the literature: the publications concentrate on gas and liquid withdrawal from large reservoirs and only correlations for the beginning of entrainment were given.

In the present investigation conditions of interest for nuclear reactors were established by:

- flow geometries without rotational symmetry,

- pipe flows dorunstream of the branch (superimposed velocities)

Circular branches with diameters of $6,8,12$ or $20 \mathrm{~mm}$, perpendicular to the main pipe with a diameter of $206 \mathrm{~mm}$, were used. The experiments were performed with air-water flow at ambient temperature and a maximum pressure of $0.5 \mathrm{MPa}$.

The flow towards the branch was observed to be vortex or vortex-free, depending on the branch orientation and superimposed velocity. Photographs are presented to illustrate the different flow phenomena.

A general correlation was developed to determine the beginning of entrainment for the three branch orientations investigated.

During entrainment, measurements were made of the branch quality and mass flux. Results are presented as a function of a normalized distance between interface and branch entrance.

A model wes developed to predict the branch quality for arbitrary fluid systems.

The results were extended to steam-water flow at high pressure with horizontal critical branch mass flux and compared with other models. 
The experiments presented in this report have improved the understanding of the flow process originating in small breaks in a horizontal pipe with stratified flow. The results can also be applied to small breaks at the bottom, the top or a side of large reservoirs and, therefore, they are useful in the development of safety codes for small break LOCA.

Further experiments with similar geometries and single component fluid system will be necessary to investigate the influence of local flashing near the interface on the beginning of entrainment and on the branch quality and mass flux. 


\section{Acknowledgements}

The author wants to express her gratitude to Dr.-Ing. J. Reimann for his numerous discussions, useful suggestions and valuable assistance during the preparation of this work. The author is also grateful to Prof. Dr.-Ing. U. Müller for his advice and stimulation. 


\section{Literature}

/1/ P.B. Walley and B.J. Azzopardi, "Two-Phase Flow in a T-Junction", AERE-R 9699, 1980.

/2/ J.A.R. Henry, "Dividing Annular Flow in a llorizontal Tee", J. Multiphase Flow, Vo1. 7 , pp 343-355, 1981.

13/ T.J. Honan and R.T. Lahey, jr., "The Measurement of Phase Separation in Wyes and Tees", Nuclear Engineering and Design, Vol. 64, No. 1, 1981.

14/ K. Zetzmann, "Phasenseparation und Druckabfall in zweiphasig durchströmten vertikalen Rohrabzweigen, Diss. Universität Hannover, 1982.

15/ N. Saba and R.T. Lahey, "Phase Separation Phenomena in Branching Conduits", NUREG/CR-2590, 1982.

/6/ J. Reimann, H.J. Brinkmann, A. Demski, H. John, L. Pawlak, W. Seeger, C. Smoglie, E. Wanner, E. Weinbrecht, "Gemeinsamer Versuchsstand zum Testen und Kalibrieren verschiedener $Z$ weiphasen-Massenstrom-Melsverfahren", PNS Jahresbericht 1982, KfK 3350, 1983, pp. 4100-94.

17/ J. Reimann, HoJ. Brinkmann, Go Eisele, H. John, L. Pawlak, W. Seeger, C. Smoglie, E. Wanner, E. Weinbrecht; IRB, "Gemeinsamer Versuchsstand zum Testen und Kalibrieren verschiedener Zweiphasenmassenstrom-Meßverfahren", PNS Jahresbericht 1983, KfK 3450, 1984, pp. 4100-131.

18/W. Seeger, "Untersuchungen zum Druckabfall und zur Massenstromumverteilung von Zweiphasenströmungen in rechtwinkligen Rohrverzweigungen", Dissertation Universität Karlsruhe, 1984.

19/ L. Dagget and G. Keulegan, "Similitude in Free Surface Vortex Formation", J. Hydr。 Div. ASCE, Vol. 100, 1506, 1974.

/10/ B. Lubin and M. Hurwitz, "Vapor Pul1-Through at a Tank Drain with and without Dielectrophoretic Buffling", Proc. Conf. Long Term Cryoprope1lant Storage in Space, NASA Marshal1 Space Center, Huntsville, Ala., p. 173., 1966. 
/11/ H. Rouse, J. Davidian, J.E. Glover and D.W. Appel, "Development of the Non-Circulatory Waterspont", J. Hydraulics Division, HY4, August 1956, Paper 1038.

112/ H. Craya, "Theoretical Research on the Flow of Non-llomogeneous Fluids", La Houille Blanche, pg. 44-55, Jan.-Feb. 1949

/13/ P. Gariel, "Experimental Research on the Flow of Non-Homogeneous Fluids", La Houille Blanche, pg. 56-64, Jan.-Feb. 1949

/14/ N. Zuber, "Problems in Modeling of Small Break LOCA", NUREG-0724, 1981

/15/ G.R. Houdayer, J.M. Miraucourt, J.C. Micaelli, "Description of Volumes in Cathare Code", European Two Phase Flow Group Meeting, Zürich, 1417 th June, 1983

/16/ C.J. Crowley and P.ll. Rothe, "Flow visualization and Break Mass Flow Measurements in Small Break Separate Effects Experiments", ANS Specialist Meeting on Small Break Loss of Coolant Accident Analyses in LWR's, Monterey, California, Aug. 25-27, 1981.

/17/ J. Reimann, M. Khan, "F1ow Through a Small Pipe at the Bottom of a Large Pipe with Stratified Flow"; Annual Meeting of the European TroPhase Flow Group, Paris 1a Defense, June 2-4, 1982.

18/ J. Reimann, M. Khan, "Flow Through a Small Pipe at the Bottom of a Large Pipe with Stratified Flow"; Second International Topical Meeting on Nuclear Reactor Thermal-Hydraulics, Santa Barbara, California, January $11-14,1983$.

/19/ H. John, J. Reimann, "Gemeinsamer Versuchsstand zum Testen und Kalibrieren verschiedener Zweiphasen-Massenstrom-Meßverfahren", KfK 2731 B, Februar 1979.

120/ J. Reimann, H. John, H.J. Brinkmann, A. Demski, L. Pawlak, W. Seeger, E. Wanner, Ro Weinbrecht, "Gemeinsamer Versuchsstand zum Testen und Ka1ibrieren verschiedener Zweiphasen-Massenstrom-Meßverfahren", PNS 4137, JB 1981, KfK 3250, 1982, pp. 4100-186. 
121/ J. Reimann, C. Smoglie, "Flow through a small pipe at the top of a large pipe with stratified flow"; Annual Meeting of the European TwoPhase Flow Group, Zürich, Switzerland, June, 14-16, 1983.

122/ J. Lighthil1, "Waves in Fluids", Cambridge University Press 1978.

123/ H. Lamb, "Hydrodynamics", Cambridge University Press 1975.

124/ Y. Taitel and A.E. Dukler, "A Model for Predicting Flow Regime Transitions in Horizontal and Near Horizontal Gas-Liquid Flow", AIChE Journa1, Vo1. 22, No. 1, Jan. 1976.

125/ Y. Taitel and A.E. Dukler, "Transient Gas-Liquid Flow in Horizontal Pipes: Modeling the Flow Pattern Transitions", AIChE Journa1, Vol. 24 , No. 5, Sept. 1978.

/26/ G.B. Wallis and J.E. Dobson, "The Onset of Slugging in Horizontal Stratified Air-Water Flow", Int. J. Multiphase Flow, Vol. 1, pp. 173-193, 1973

/27/ Wen-Hsiung Li, Sau-Hai Lam, "Principles of Fluid Mechanics", AddisonWesely Publishing Company, 1964.

128/ C. Smog1ie, J. Reimann, "Two-Phase Flow Through a Small Horizontal Branch in a Pipe with Stratified Flow", Jahrestagung Kerntechnik '84, Frankfurt 22-24 Mai 1984.

/29/ J. Reimann, Not published data.

130/ J. Weisbach, "Die Experimentalhydraulik", J.S. Engelhrdt, Freiburg 1855.

/31/ T.B. Drew, E.C. Koo and W.H. McAdams, "The Friction Factor in Smooth Wall Turbulent Flow", Trans., AIChE 28, 56 (1932).

/32/ V.L. Streeter, "Handbook of Fluid Dynamics", Chapter 3, McGraw-Hi11 Book Co. Inc., 1961. 
133/ VDI 2040, "Berechnungsgrundlagen für die Durchflußmessung von Drosse1geräten", VDI-Ver1ag, Düsseldorf, 1971.

/34/ F. D'Auria, P. Vigni, "Two Phase Critical Flow Models", CSNI Report No. 49,1980 .

135/ F.J. Moody, "Maximum Flow Rate of a Single Component, Two-Phase Mixture", Transactions of the ASME, JHT, 1965, 87, pp. 134-142.

/36/ G.B. Wallis, "Critical Two Phase Flow", Int. J. Multiphase Flow, Vol. 6, pp. $97-112,1980$.

137 / G.G. Weigand, S.L. Thompson, D. Tomasko, "Two Phase Jet Loads", NUREG/CR-2913, SAND 82-1935, R4, Jan. 1983.

/38/ K.R. Katsma et a1., "User's Manual for RELAP4/MOD.5", INEL-Report, ANCR-NUREG-1335, Sept. 76.

/39/ V.H. Ransom, R.J. Wagner, J.A. Trapp, K.E. Carlson, D.M. Kiser, H.H. Kuo, H. Chow, R.A. Ne1son, S.W. James, "RFLAP5/MODl Code Manual Vol.1: System Models and Numerical Methods", NUREG/CR-1826 EGG-2070 DRAFT Revision 1, March 1981. 


\section{Appendix}

\section{A1 Method used to Calculate Propagated Errors}

The error bars appearing in a number of results presented in this report, were determined by estimating the relative error of the measured parameters and the corresponding propagated error of the calculated variables.

The errors in the measured parameters mostly depend on the flow configuration and range of operation of the instruments, therefore the accuracy of the measurements was estimated for each typical experiment.

The relative error in the calculated variables was determined according to the theory of error propagation:

- The relative error $\delta M$ of a magnitude $M$ measured with an absolute error $\Delta M$ is :

$$
\delta M=\frac{\Delta M}{M}
$$

- The relative error of a function $F\left(M_{i}\right)$ of $n$ variables $M_{i}$ measured with absolute errors $\Delta M_{i}$, is:

$$
\delta F \equiv \frac{\Delta F}{F}=\frac{1}{F} \sum_{i=1}^{n} \frac{\partial F}{\partial M_{i}} \Delta M_{i}
$$

Equations (1.1) and (1.2) were used with the following conditions:

- No error was asigned to $\left.\mathrm{D}, \mathrm{d}, \mathrm{A}_{1}, \mathrm{~A}_{3}, \mathrm{~g}, \varepsilon, \rho_{1}, \mathrm{k},\right\}, \mathrm{c}_{\mathrm{a}}, \mathrm{c}_{g}$, and $\mathrm{c}_{1}$.

- The relative error in $A_{g}$ and $A_{1}$ was determined considering the absolute error in $h_{g}$ and $h_{1}$ respectively and using a table for areas of circular sectors.

This procedure gives the formulas listed below:

$$
\begin{aligned}
& \rho_{g}=c_{a} \frac{P_{1}}{T_{1}} \\
& \delta \rho_{g}=\delta P_{1}+\delta T_{1} \\
& \delta\left(\rho_{1}-\rho_{g}\right)=\frac{\rho_{g}}{\rho_{e}-\rho_{g}} \delta \rho_{g}
\end{aligned}
$$




$$
\begin{aligned}
& W_{(1,2,3) g}=c_{g}\left(P_{o} \Delta P_{o} T_{o}\right)^{0.5} \\
& \delta \mathrm{W}_{(1,2,3) \mathrm{g}}=0.5\left(\delta \mathrm{P}_{\mathrm{o}}+\delta \Delta \mathrm{P}_{\mathrm{o}}+\delta \mathrm{T}_{\mathrm{o}}\right) \\
& \mathrm{w}_{11}=\mathrm{c}_{1}\left(\rho_{1} \Delta \mathrm{P}_{\mathrm{o}}\right)^{0.5} \\
& \delta_{\mathrm{W}_{11}}=0.5 \delta \Delta_{\mathrm{P}_{\mathrm{o}}} \\
& w_{3 g}=w_{1 g}-w_{2 g} \\
& \delta_{W_{3 g}}=\frac{1}{W_{3 g}}\left(W_{1 g} \delta_{W_{1 g}}-W_{2 g} \delta_{W_{2 g}}\right) \\
& \text { ' } W_{31}=\text { weight of water/time } \\
& \delta_{w_{31}}=\delta_{\text {weight }}+\delta_{\text {time }} \\
& v_{1 g}=\frac{W_{1 g}}{\rho_{g} A_{g}} \\
& \delta v_{1 g}=\delta W_{1 g}+\delta \rho_{g}+\delta_{A_{g}} \\
& h_{b}=\left[K\left(\zeta E A_{3}\right)^{0.4} / g^{0.2}\right]\left(\Delta P_{1-34} / \rho_{1}-\rho_{g}\right)^{0.2} \\
& \delta h_{b}=0.2\left[\delta \Delta P_{1-34}+\delta\left(\rho_{1}-\rho_{g}\right)\right] \\
& x_{3}=\frac{1}{1+W_{31} / w_{3 g}} \\
& \delta_{x_{3}}=\left(1-x_{3}\right)\left(\delta w_{31}+\delta w_{3 g}\right) \\
& G_{3}=\left(W_{3 g}+W_{31}\right) / A_{3} \\
& \delta_{G_{3}}=x_{3} \delta_{W_{3 g}}+\left(1-x_{3}\right) \delta_{W_{31}} \\
& \left.G_{3 b}=\right\} \varepsilon\left(\rho_{b} \Delta P_{1-34}\right)^{0.5} \\
& \delta G_{3 b}=0.5\left(\delta \rho_{b}+\delta \Delta P_{1-34}\right)
\end{aligned}
$$


The estimated errors in the measurements and their contribution to the propagated error in the results are illustrated below with two numerical examples.

Example 1: Beginning of gas entrainment in downward branches: strong vorticity. Measured parameters:

$$
\begin{aligned}
& \mathrm{P}_{1}=(4.1 \pm 0.1) \mathrm{bar} \\
& \mathrm{T}_{1}=(290 \pm 1.5) \mathrm{K} \\
& \mathrm{h}_{\mathrm{b}}=(33 \pm 1.5) \mathrm{mm} \\
& \text { weight of water }=(54.5 \pm 0.1) \mathrm{kg} \\
& \text { time }=(427 \pm 3) \mathrm{sec}
\end{aligned}
$$

Plotted variables: $\mathrm{K}_{\mathrm{ge}}=\mathrm{h}_{\mathrm{b}}\left[\mathrm{g} \rho_{1}\left(\rho_{1}-\rho_{g}\right)\right]^{0.2} / \mathrm{w}_{31} 0.4 ; \mathrm{h}_{\mathrm{b}} / \mathrm{d}$

Propagated errors:

$$
\begin{aligned}
\delta_{K_{g e}} & =\delta_{h_{b}}+0.2 \delta\left(\rho_{1}-\rho_{g}\right)+0.4 \delta_{W_{31}} \\
& =\delta_{h_{b}}+0.2 \frac{\rho_{g}}{\rho_{\ell-\rho_{g}}}\left(\delta_{P_{1}}+\delta_{T_{1}}\right)+0.4\left(\delta_{\text {weight }}+\delta_{\text {time }}\right) \\
& =\frac{1.5}{33}+0.2 \frac{4.9}{995.1}\left(\frac{0.1}{4.1}+\frac{1.5}{290}\right)+0.4\left(\frac{0.1}{54.5}+\frac{3}{427}\right) \\
\delta_{k_{g e}} & =0.045+0.00003+0.0035 \simeq 4.9 \% \\
\delta\left(h_{b} / d\right) & =\delta_{h_{b}}=0.045=4.5 \%
\end{aligned}
$$

Similar results are obtained for the beginning of entrainment in other geometries. Therefore the relative error in $\mathrm{K}$ is practically given by the relative error in the liquid level.

Example 2: Liquid entrainment in upward branches: wavy interface. Maximal $\delta_{\mathrm{w}_{31}}$ due to water evaporation: $20 \%$ Measured parameters:

$$
\begin{aligned}
& \mathrm{P}_{\mathrm{OJ}}=(14.3 \pm 0.1) \mathrm{bar} \\
& \mathrm{T}_{\mathrm{OI}}=(289 \pm 1.5) \mathrm{K} \\
& \Delta \mathrm{P}_{\mathrm{O}}=(60 \pm 3) \mathrm{mmHg} \\
& \mathrm{P}_{1}=(4.20 \pm 0.05) \mathrm{bar} \\
& \mathrm{T}_{1}=(289 \pm 1.5){ }^{\circ} \mathrm{C} \\
& \Delta \mathrm{P}_{1-34}=(0.12 \pm 0.01) \text { bar } \\
& \mathrm{h}=(61 \pm 2) \mathrm{mm}
\end{aligned}
$$


Plotted variables:

$$
\begin{aligned}
& \frac{h}{h_{b}}=h /\left[K\left(\left\{\varepsilon A_{3}\right)^{0.4} / g^{0.2}\right]\left(\Delta P_{1-34} /\left(\rho_{1}-\rho_{g}\right)\right)^{0.2}\right. \\
& x_{3}=1 /\left(1+w_{31} / w_{3 g}\right) \simeq 0.991 \\
& \left.\frac{G_{3}}{G_{3 b}}=\left(w_{3 g}+w_{31}\right) / A_{3}\right\} \varepsilon\left(\rho_{b} \Delta P_{1-34}\right)^{0.5}
\end{aligned}
$$

Propagated errors:

$$
\begin{aligned}
\delta \frac{h}{h_{b}} & =\delta h+0.2\left[\delta \Delta P_{1-34}+\delta\left(\rho_{1}-\rho_{g}\right)\right] \\
& =\delta h+0.2\left[\delta \Delta P_{1-34}+\frac{\rho_{g}}{\rho_{l}-\rho_{g}}\left(\delta P_{1}+\delta T_{1}\right)\right] \\
& =\frac{2}{61}+0.2 \cdot\left[\frac{0.01}{0.12}+\frac{5.1}{994.9}\left(\frac{0.05}{4.20}+\frac{1.5}{289}\right)\right]
\end{aligned}
$$$$
\delta \frac{h}{h_{b}}=0.0328+0.0168=5 \%
$$$$
\delta x_{3}=\left(1-x_{3}\right)\left(\delta w_{31}+\delta w_{3 g}\right)
$$$$
=(1-0.991)\left[0.20+0.5\left(\delta \mathrm{P}_{01}+\delta \Delta \mathrm{P}_{01}+\delta \mathrm{T}_{\mathrm{o1}}\right)\right]
$$$$
=0.009\left[0.20+\frac{3}{350}+0.5\left(\frac{0.1}{14.3}+\frac{2}{60}+\frac{1.5}{289}\right)\right]
$$

$$
\begin{aligned}
\delta_{x_{3}} & =0.002=0.2 \% \\
\delta \frac{G_{3 b}}{G_{3 b}} & =x_{3} \delta_{W_{3 g}}+\left(1-x_{3}\right) \delta_{W_{31}}+0.5\left(\delta \rho_{g}+\delta \Delta P_{1-34}\right) \\
& =0.991 \times 0.5\left(\delta P_{01}+\delta \Delta P_{01}+\delta T_{01}\right)+0.009 \times 0.2+0.5\left(\delta_{P_{1}}+\delta T_{1}+\delta \Delta P_{1-34}\right) \\
& =0.5\left(\frac{0.1}{14.3}+\frac{2}{60}+\frac{1.5}{289}+0.009 \times 0.2+0.5\left(\frac{0.05}{4.20}+\frac{1.5}{289}+\frac{0.01}{0.12}\right.\right.
\end{aligned}
$$

From these results the following conclusions are valid for general entrainment processes:

- The relative error in $h / h_{b}$ is dominated by the relativ error in $h$.

- The relative error in $x_{3}$ increases for decreasing qualities and, for upward branches, is dominated by the error in $W_{31}$ due to liquid evaporation.

- The main contribution to the relative error in $G_{3} / G_{3 b}$ are the errors in $\Delta \mathrm{P}_{1-34}$ and $\mathrm{w}_{3 \mathrm{~g}}$ or $\mathrm{W}_{31}$ for high or low qualities, respectively. 
A2 Theoretical Research on the F1ow of Nonhomogeneous Fluids (A. Craya/12/)

Figure 2.1 defines the parameters used by Craya to determine the incipient liquid entrainment through a side orifice above the horizontal interface between two incompressible fluids of different densities.

Fig. 2.1

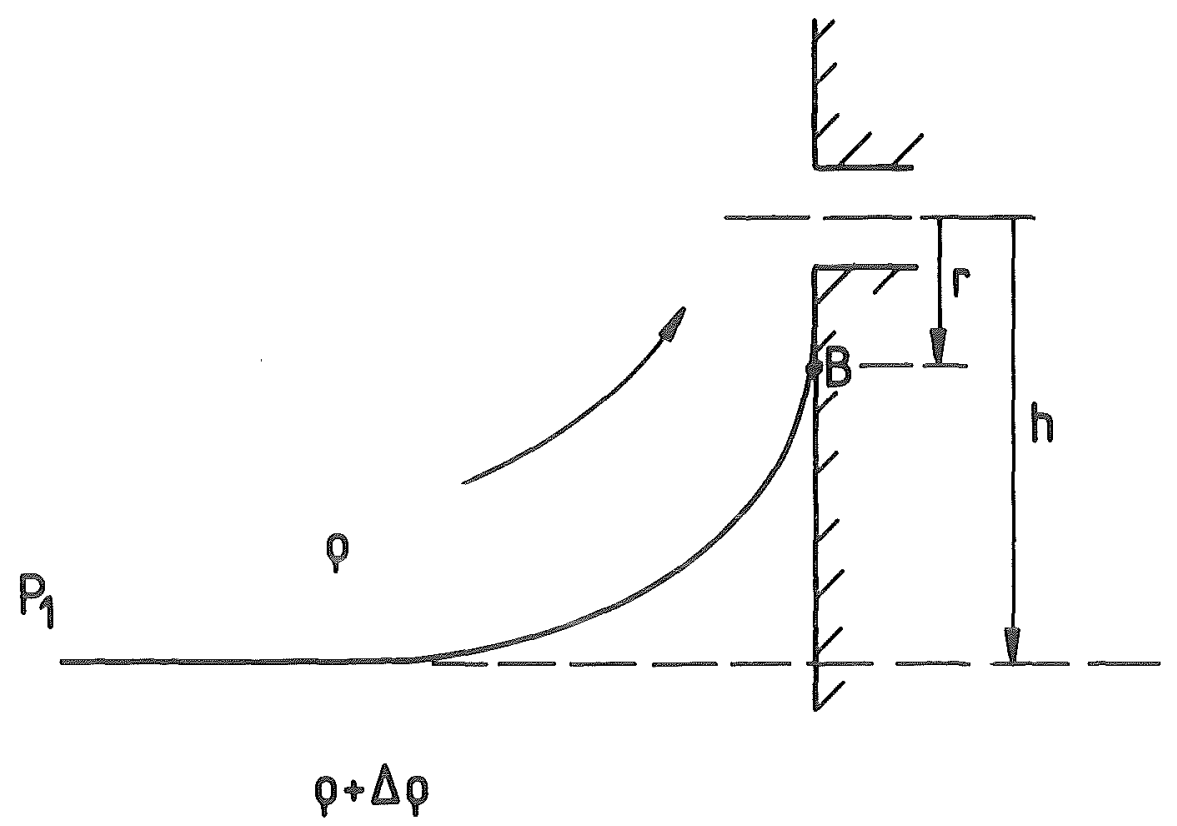

The heavier fluid, at rest, satisfies the hydrostatic condition:

$$
P_{B}+g(\rho+\Delta \rho)(h-r)=P_{1}
$$

where $r$ is the distance from the orifice axis to the top $B$ of the deflected interface. The lighter fluid, entering the orifice with phase velocity $V$, satisfies the Bernouil1i equation:

$$
P_{B}+1 / 2 \rho V^{2}+g \rho(h-r)=P_{1}
$$

From Eqs. (2.1) and (2.2) the condition of equilibrium at $B$ is:

$$
\frac{V^{2}}{2}=\frac{\Delta P}{p} g(h-r)
$$

If the velocity $\vec{V}$ verifies the condition for a two dimensional sink of strength q:

$$
V=\frac{q}{\pi r}
$$


$\mathrm{Eq} \cdot(2.3)$ becomes

$$
\frac{v^{2}}{2}=\frac{J \rho}{\rho} g(h-r)=\frac{q^{2}}{2 \pi^{2} r^{2}}
$$

From this expression the term $v^{2} / 2$ can be plotted as a function of $r$ with two different curves: a straight line and a parabola, as shown in Fig. 2.2.

Fig. 2.2

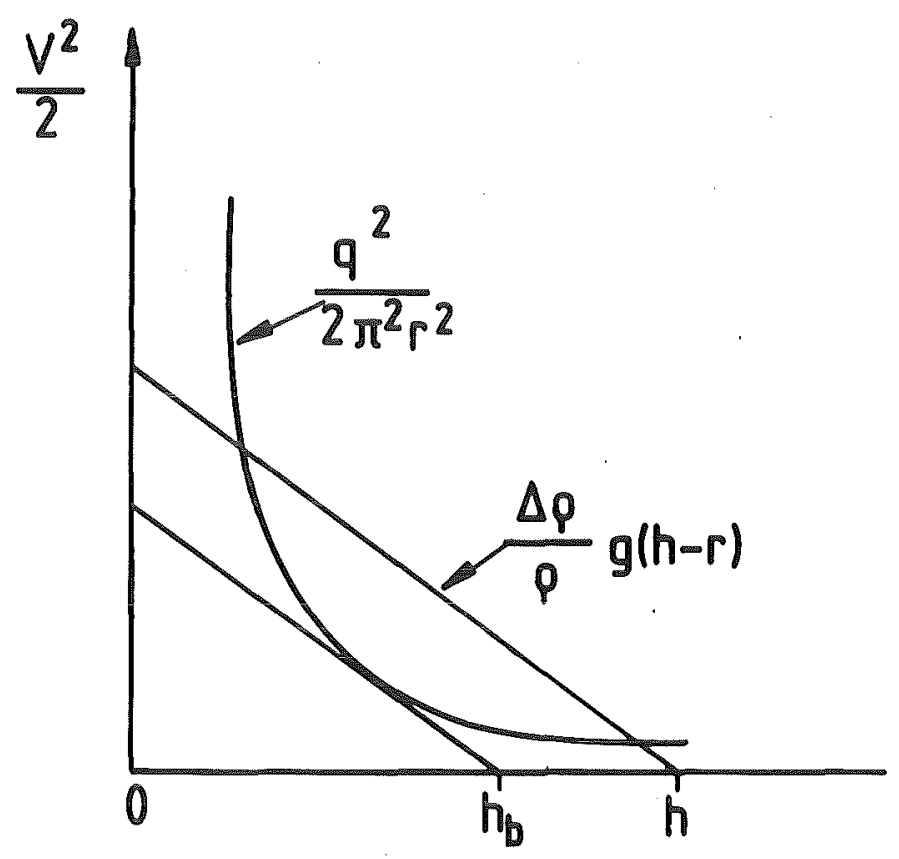

The condition for the beginning of entrainment is obtained when Eq. (2.5) has a double root, $i_{\circ} e_{\circ}$, for $r=2 h_{b} / 3$ which, replaced in (2.5), gives:

or

$$
\frac{\Delta p}{p}=g\left(h_{6}-\frac{2}{3} h_{b}\right)=q^{2} / 2 \pi^{2}\left(2 h_{6} / 3\right)^{2}
$$

$$
\frac{\Delta p}{p_{6}} g \frac{h_{6}^{3}}{q^{2}}=27 / 8 \pi^{2}
$$

Craya extends this result to a three dimensional punctual sink just writing:

$$
\frac{\Delta p}{p_{b}} g \frac{h_{b}^{5}}{q^{2}}=\text { constant }
$$

and obtains finally the value 0.154 for the constant. 


\section{A3 The Homogeneous Equlibrium Mode1 (HEM) (F.J. Moody /35/)}

The theory known as HEM (Homogeneous Equilibrium Model) is the most simple equilibrium model that may be formulated in the analysis of two phase flow (compare D'Auria et a1. /34/). It has been developed initially to analyze situations in which a fluid contained in a pressure vessel outflows through a pipe the diameter of which is much smaller than the vessel diameter.

The basic assumptions of the HEM are:

- llomogeneity: The mixture is considered as a single component fluid with channel averaged thermodynamical properties and both phases having the same velocity.

- Thermodynamical equilibrium: The pressure and temperature of liquid and vapor are equal and take values corresponding to the Mollier diagram saturation curve. However, changes of the quality are allowed along the flow path, which is in contrast with the thermodynamical hypothesis, because condensation and evaporation depend on differences of temperature and/or pressure through the phases. Implicitly, the changes in quality are supposed to take place at infinite velocity.

- Isentropic flow: Neither heat nor work is interchanged with the exterior.

For stationary flow, the balance equations are:

- continuity equation:

$$
P_{\mathrm{m}} \mathrm{VA}=\text { const } .
$$

- energy equation:

$$
1 / 2 \rho_{m} v^{2}+h=h_{0}
$$

- state equations:

$$
\begin{aligned}
& \mathrm{h}=\mathrm{h}_{1}+\mathrm{x} \mathrm{h}_{1 \mathrm{~g}} \\
& \mathrm{v}_{\mathrm{m}}=\mathrm{v}_{1}+\mathrm{x} \mathrm{v}_{1 \mathrm{~g}} \\
& \mathrm{~s}=\mathrm{s}_{1}+\mathrm{x} \mathrm{s}_{1 \mathrm{~g}}
\end{aligned}
$$

- isentropic conditions:

$$
\mathrm{d} s=0
$$

By combining the equations above the following expression is obtained for the mass flux:

$$
G=\frac{2\left[h_{0}-h_{l}-\frac{s_{0}-5}{s_{e g}} h_{l g}\right]^{0.5}}{v_{l}+\frac{s_{0}-s_{l}}{s_{l g}} v_{l g}}
$$


After giving the reservoir conditions the mass flux given by Eq. (3.7) depends only upon the thermodynamic state of the fluid. Consequently, the maximum mass flux (critical flow) must also depend only on the thermodynamic state of the fluid.

Choking is defined as the state in the fluid where changes in the downstream properties do not alter the mass flow rate or any properties upstream of the break. The critical mass flux G crit occurs at the point where the flow reaches the sonic velocity:

$$
\mathrm{V} / \mathrm{c}=1
$$

To determine $G_{\text {crit }}$ the downstream pressure is decreased until the mass flux reachs its maximal value. 
This model assumes stratified flow in a horizontal main pipe of diameter $D$ (void fraction $\alpha_{1}$ ) and choked flow in a horizontal branch of diameter $d$ (void fraction $\alpha_{3}$ ). To define $\alpha_{3}$ as a function of $\alpha_{1}$ the RELAP5 code uses the criterion for transition from stratified horizontal flow regime in a round pipe as given by Taitel and Dukler /24, 25/:

$$
v_{g}>\left(1-h_{1} / D\right)\left[g\left(\rho_{\rho}-P_{g}\right) / \rho_{g} A_{\rho}^{\prime}\right]^{0.5}
$$

where the symbols are those defined in Section 4.4. The right hand side of $\mathrm{Eq} \cdot(4.1)$ is called the limit gas velocity $\mathrm{gL}$.

The central angle $\theta=\cos ^{-1}\left(2 h_{1} / D-1\right)$, defined in Fig. 4.1

Fig. 4.1

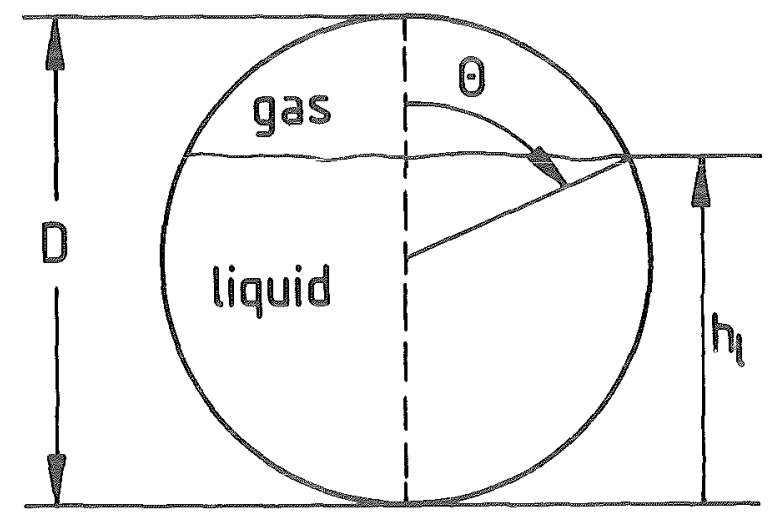

is used to rewrite $h_{1}, A_{1}$ and $\alpha_{1}$ as follows:

$$
\begin{aligned}
& h_{1}=D / 2(1+\cos \theta) \\
& A_{1}^{\prime}=D \sin \theta \\
& a_{1}=(2 \theta-\sin 2 \theta) / 2 \pi
\end{aligned}
$$

where $\theta$ is expressed in radians.

With $A_{g}=d_{1} \pi D^{2} / 4$ and the expressions given by Eqs. (4.2), (4.3) and (4.4), the limit gas velocity (Eq. $(4.1)$ ) becomes:

$$
v_{g L}=\frac{1}{4 \sqrt{2}}(1-\cos \theta)\left[D g \frac{\rho_{l}-\rho_{g}}{\rho_{g}} \frac{2 \theta-\sin 2 \theta}{\sin \theta}\right]^{0.5}
$$

The RELAP5 code defines $\alpha_{3}$ for the interface above and below the branch entrance, respectively, as follows: 


$$
\begin{array}{ll}
\alpha_{3}=\alpha_{1}\left(v_{\text {ig }} / v_{g L}\right)^{0.5} & \text { for } h_{1}>(D+d) / 2 \\
\alpha_{3}=1-\left(1-\alpha_{1}\right)\left(v_{i g} / v_{g L}\right)^{0.5} & \text { for } h_{1}<(D-d) / 2
\end{array}
$$

For liquid levels within the outlet area, i.e., for $(D-d) / 2<h_{1}<(D+d) / 2$, $\alpha_{3}$ is obtained by interpolation of the two void fractions computed at the boundaries. 
$-111-$

A5 TABLES OF EXPERIMENTAL DATA 
Table 5.1: Transition from stratitied to slug flow in the horizontal main pipe (upward branches)

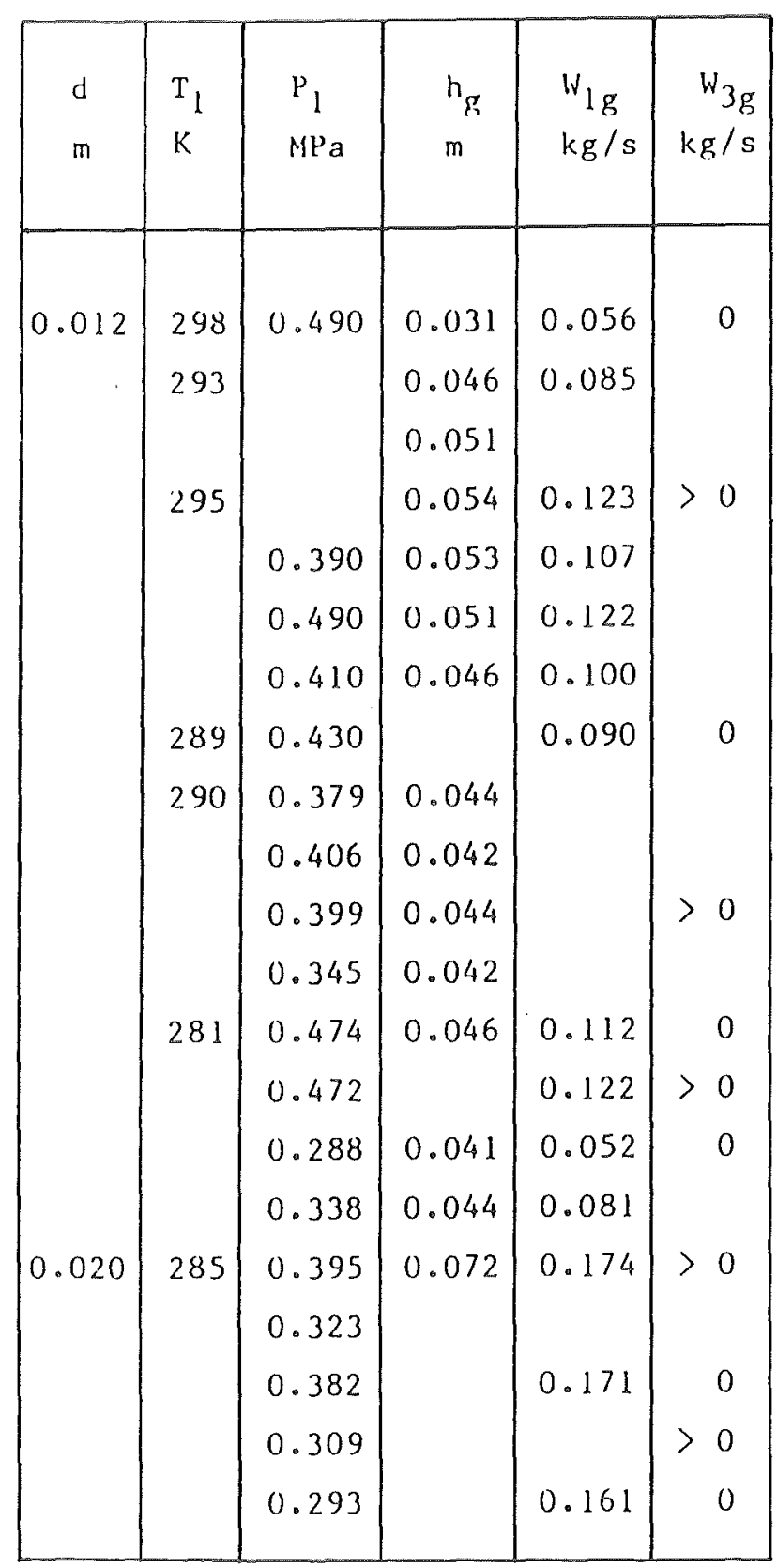

\begin{tabular}{|c|c|c|c|c|c|}
\hline $\begin{array}{l}\mathrm{d} \\
\mathrm{m}\end{array}$ & $\begin{array}{l}T_{1} \\
K\end{array}$ & $\begin{array}{r}P_{1} \\
\mathrm{MPa}\end{array}$ & $\underset{\mathrm{m}}{\mathrm{h}_{\mathrm{g}}}$ & $\begin{array}{r}W_{1 \mathrm{~g}} \\
\mathrm{~kg} / \mathrm{s}\end{array}$ & $\begin{array}{r}W_{3 g} \\
\mathrm{~kg} / \mathrm{s}\end{array}$ \\
\hline 0.020 & 282 & $\begin{array}{l}0.272 \\
0.250 \\
0.238 \\
0.500 \\
0.498 \\
0.475 \\
0.408 \\
0.482 \\
0.358 \\
0.341 \\
0.322 \\
0.423 \\
0.366 \\
0.394 \\
0.373 \\
0.235 \\
0.404 \\
0.421 \\
0.447 \\
0.393 \\
0.460\end{array}$ & $\begin{array}{l}0.048 \\
0.042 \\
0.042 \\
0.060 \\
0.052 \\
0.056 \\
0.057 \\
0.061 \\
0.065 \\
0.066\end{array}$ & $\begin{array}{l}0.278 \\
0.181 \\
0.154 \\
0.166 \\
0.091 \\
0.082\end{array}$ & $\begin{aligned}> & 0 \\
& 0 \\
> & 0 \\
& 0 \\
> & 0 \\
& \\
& 0 \\
> & 0 \\
& 0 \\
> & 0 \\
& 0\end{aligned}$ \\
\hline
\end{tabular}


Table 5.2: Beginning of gas entrainment in downward branches; $v_{2 g}=0, v_{21}=0$.

\begin{tabular}{|c|c|c|c|c|c|c|c|c|c|}
\hline $\begin{array}{l}d \\
m\end{array}$ & $\begin{array}{l}\mathrm{T}_{1} \\
\mathrm{~K}\end{array}$ & $\begin{array}{l}P_{1} \\
\mathrm{MPa}\end{array}$ & $\begin{array}{l}h_{b} \\
m\end{array}$ & $\begin{array}{r}W_{3 b} \\
\mathrm{~kg} / \mathrm{s}\end{array}$ & $\begin{array}{l}d \\
m\end{array}$ & $\begin{array}{r}\mathrm{T}_{1} \\
\mathrm{~K} \\
\end{array}$ & $\begin{array}{l}\mathrm{P}_{1} \\
\mathrm{MPa}\end{array}$ & $\begin{array}{l}\mathrm{h}_{\mathrm{b}} \\
\mathrm{m}\end{array}$ & $\begin{array}{r}W_{3 b} \\
\mathrm{~kg} / \mathrm{s}\end{array}$ \\
\hline \multirow[t]{9}{*}{0.006} & \multirow[t]{9}{*}{291} & 0.412 & 0.055 & 0.281 & \multirow[t]{7}{*}{0.008} & \multirow[t]{7}{*}{294} & 0.435 & 0.063 & 0.383 \\
\hline & & 0.417 & 0.060 & 0.367 & & & 0.441 & & 0.444 \\
\hline & & 0.415 & 0.066 & 0.454 & & & 0.434 & & 0.524 \\
\hline & & 0.414 & 0.068 & 0.473 & & & 0.433 & 0.074 & 0.697 \\
\hline & & 0.412 & 0.030 & 0.128 & & & 0.421 & 0.034 & 0.128 \\
\hline & & \multirow[t]{3}{*}{0.419} & 0.040 & 0.182 & & & 0.433 & 0.025 & 0.073 \\
\hline & & & 0.056 & 0.315 & & & 0.404 & 0.070 & 0.657 \\
\hline & & & 0.060 & 0.381 & 0.012 & 296 & 0.438 & 0.058 & 0.428 \\
\hline & & 0.409 & 0.045 & 0.236 & & & 0.420 & 0.070 & 0.623 \\
\hline \multirow[t]{12}{*}{0.008} & \multirow[t]{4}{*}{290} & 0.414 & 0.047 & 0.221 & & & 0.443 & 0.075 & 0.721 \\
\hline & & 0.412 & 0.041 & 0.280 & & & 0.463 & 0.080 & 0.975 \\
\hline & & 0.399 & 0.057 & 0.265 & & & 0.423 & 0.085 & 1.391 \\
\hline & & 0.399 & 0.061 & 0.519 & & & 0.482 & 0.083 & 1.020 \\
\hline & \multirow[t]{5}{*}{295} & 0.478 & 0.054 & 0.351 & & & 0.412 & 0.056 & 0.518 \\
\hline & & 0.381 & 0.040 & 0.202 & & & 0.439 & 0.048 & 0.261 \\
\hline & & 0.406 & 0.064 & 0.465 & & & 0.431 & 0.061 & 0.446 \\
\hline & & 0.370 & 0.073 & 0.632 & & & 0.438 & 0.070 & 0.631 \\
\hline & & 0.337 & 0.077 & 0.733 & & 291 & 0.490 & 0.050 & 0.366 \\
\hline & \multirow[t]{3}{*}{294} & 0.474 & 0.045 & 0.257 & & & 0.414 & 0.046 & 0.288 \\
\hline & & 0.423 & 0.041 & 0.113 & & & & 0.033 & 0.128 \\
\hline & & 0.433 & 0.046 & 0.283 & & & & & \\
\hline
\end{tabular}


Table 5.3: Beginning of 1iquid entrainment in upward branches; $v_{2 g}=0, v_{21}=0$

\begin{tabular}{|c|c|c|c|c|}
\hline $\begin{array}{l}d \\
m\end{array}$ & $\begin{array}{l}T_{1} \\
K\end{array}$ & $\begin{array}{l}\mathrm{P}_{1} \\
\mathrm{MPa}\end{array}$ & $\begin{array}{l}h_{b} \\
m\end{array}$ & $\begin{array}{c}w_{3 b} \\
10^{-3} \mathrm{~kg} / \mathrm{s} \\
\end{array}$ \\
\hline \multirow[t]{15}{*}{0.006} & 285 & 0.463 & 0.034 & 9.9 \\
\hline & & 0.448 & 0.041 & 13.2 \\
\hline & & 0.463 & 0.043 & 15.8 \\
\hline & & 0.462 & 0.049 & 19.7 \\
\hline & & 0.472 & 0.026 & 7.1 \\
\hline & & 0.461 & 0.030 & 8.15 \\
\hline & & 0.485 & 0.032 & 9.0 \\
\hline & & 0.484 & 0.038 & 11.7 \\
\hline & & 0.470 & 0.044 & 15.7 \\
\hline & 286 & 0.343 & 0.030 & 6.8 \\
\hline & & 0.462 & 0.032 & 9.0 \\
\hline & & 0.507 & 0.035 & 10.0 \\
\hline & & 0.455 & 0.038 & 10.5 \\
\hline & & 0.343 & 0.042 & 11.3 \\
\hline & & 0.417 & 0.045 & 14.3 \\
\hline \multirow[t]{18}{*}{0.012} & 298 & 0.320 & 0.068 & 46.0 \\
\hline & & 0.340 & 0.061 & 55.7 \\
\hline & 288 & 0.510 & 0.081 & 114.4 \\
\hline & 293 & 0.380 & 0.083 & 85.2 \\
\hline & 295 & 0.510 & 0.086 & 130.9 \\
\hline & & 0.380 & 0.074 & 71.1 \\
\hline & & 0.350 & 0.064 & 52.0 \\
\hline & & & 0.054 & 33.9 \\
\hline & 292 & 0.500 & 0.061 & 40.8 \\
\hline & & 0.490 & 0.057 & 33.1 \\
\hline & & & 0.054 & 24.1 \\
\hline & & 0.470 & 0.052 & 19.0 \\
\hline & & 0.500 & 0.048 & 29.3 \\
\hline & & 0.480 & 0.045 & 23.8 \\
\hline & & 0.430 & 0.048 & 17.4 \\
\hline & & 0.370 & 0.037 & 11.7 \\
\hline & & 0.287 & 0.1131 & 10.4 \\
\hline & & 0.490 & 0.079 & 109.2 \\
\hline
\end{tabular}

\begin{tabular}{|c|c|c|c|c|}
\hline $\mathrm{m}$ & $\begin{array}{c}T_{1} \\
k\end{array}$ & $\begin{array}{l}\mathrm{P}_{1} \\
\mathrm{MPa}\end{array}$ & $\begin{array}{l}h_{b} \\
m\end{array}$ & $\begin{array}{c}w_{3 b} \\
10^{-3} \mathrm{~kg} / \mathrm{s}\end{array}$ \\
\hline \multirow[t]{20}{*}{0.012} & \multirow[t]{2}{*}{292} & 0.520 & 0.081 & 118.9 \\
\hline & & 0.420 & & 95.9 \\
\hline & \multirow[t]{4}{*}{290} & 0.208 & 0.054 & 40.6 \\
\hline & & 0.482 & 0.066 & 39.4 \\
\hline & & 0.434 & 0.076 & 87.0 \\
\hline & & 0.479 & & 104.8 \\
\hline & \multirow[t]{14}{*}{291} & 0.385 & 0.072 & 92.7 \\
\hline & & 0.407 & 0.073 & \\
\hline & & 0.471 & 0.074 & 85.6 \\
\hline & & $0.390^{\circ}$ & 0.062 & 92.7 \\
\hline & & 0.324 & & 74.5 \\
\hline & & 0.508 & 0.066 & 59.8 \\
\hline & & 0.431 & & 97.7 \\
\hline & & 0.219 & 0.046 & 48.2 \\
\hline & & 0.487 & 0.050 & 32.7 \\
\hline & & & & 43.0 \\
\hline & & 0.479 & 0.069 & 75.0 \\
\hline & & 9.345 & 0.072 & 73.5 \\
\hline & & 0.469 & 0.073 & 93.0 \\
\hline & & 0.478 & 0.079 & 109.6 \\
\hline \multirow[t]{14}{*}{0.020} & \multirow[t]{9}{*}{288} & 0.490 & 0.064 & 181.0 \\
\hline & & 0.450 & & 154.0 \\
\hline & & 0.350 & & 128.0 \\
\hline & & 0.300 & & 116.0 \\
\hline & & 0.250 & & 97.0 \\
\hline & & 0.490 & 0.066 & 29.0 \\
\hline & & 0.430 & & 62.0 \\
\hline & & 0.300 & & 42.0 \\
\hline & & 0.490 & 0.067 & 105.0 \\
\hline & \multirow[t]{5}{*}{292} & 0.510 & 0.072 & 174.0 \\
\hline & & 0.380 & & 171.0 \\
\hline & & 0.290 & & 161.0 \\
\hline & & 0.250 & & 143.0 \\
\hline & & (1).220) & & 127.0 \\
\hline
\end{tabular}


Table 5.3: (2nd page)

\begin{tabular}{|c|c|c|c|c|}
\hline $\begin{array}{l}d \\
m\end{array}$ & $\begin{array}{r}\mathrm{T}_{1} \\
\mathrm{~K}\end{array}$ & $\begin{array}{l}\mathrm{P}_{1} \\
\mathrm{MPa}\end{array}$ & $\begin{array}{l}h_{b} \\
m\end{array}$ & $\frac{W_{3} \mathrm{~b}}{10^{-3} \mathrm{~kg} / \mathrm{s}}$ \\
\hline \multirow[t]{14}{*}{0.020} & 292 & 0.180 & 0.072 & 99 \\
\hline & 288 & 0.350 & 0.073 & 82 \\
\hline & & 0.470 & & 106 \\
\hline & & 0.490 & 0.076 & 242 \\
\hline & & 0.500 & & 278 \\
\hline & & 0.390 & & 106 \\
\hline & & 0.360 & & 124 \\
\hline & & 0.410 & & 167 \\
\hline & 292 & 0.470 & 0.080 & 183 \\
\hline & 288 & 0.490 & 0.086 & 278 \\
\hline & 292 & 0.190 & 0.089 & 99 \\
\hline & & 0.210 & & 120 \\
\hline & & 0.230 & & 136 \\
\hline & & 0.260 & & 150 \\
\hline
\end{tabular}

\begin{tabular}{|c|c|c|c|c|}
\hline $\mathrm{d}$ & $\mathrm{T}_{1}$ & $\mathrm{P}_{1}$ & $\mathrm{~h}_{\mathrm{b}}$ & $\mathrm{W}_{3 \mathrm{~b}}$ \\
$\mathrm{~m}$ & $\mathrm{k}$ & $\mathrm{MPa}^{-3} \mathrm{mg} / \mathrm{s}$ \\
\hline 0.020 & 288 & 0.470 & 0.095 & 297 \\
& & & & 322 \\
& 293 & 0.466 & 0.060 & 38 \\
& & 0.514 & 0.061 & 71 \\
& \multirow{6}{*}{292} & 0.507 & 0.068 & 86 \\
& & 0.475 & & 57 \\
& & 0.388 & 0.071 & 81 \\
& & 0.516 & & 102 \\
& & 0.393 & & 71 \\
& 293 & 0.504 & 0.075 & 118 \\
& 292 & 0.383 & 0.077 & 103 \\
& 293 & 0.485 & 0.079 & 154 \\
& & 0.292 & & 87 \\
\hline
\end{tabular}


Table 5.4: Beginning of liquid entrainment in horizontal branches; $v_{2 g}=0, v_{21}=0$.

\begin{tabular}{|c|c|c|c|c|c|c|c|c|c|}
\hline$m$ & $\begin{array}{r}\mathrm{T}_{1} \\
\mathrm{~K}\end{array}$ & $\begin{array}{l}\mathrm{P}_{1} \\
\mathrm{MPa}\end{array}$ & $h_{b}$ & $\begin{array}{c}w_{3 b} \\
10^{-3} \mathrm{~kg} / \mathrm{s}\end{array}$ & $\begin{array}{l}d \\
m\end{array}$ & $\begin{array}{l}\mathrm{T}_{1} \\
\mathrm{~K}\end{array}$ & $\begin{array}{l}\mathrm{P}_{1} \\
\mathrm{MPa}\end{array}$ & $\begin{array}{l}\mathrm{h}_{\mathrm{b}} \\
\mathrm{m}\end{array}$ & $\begin{array}{l}W_{3 b} \\
\mathrm{~kg} / \mathrm{s}\end{array}$ \\
\hline \multirow[t]{19}{*}{0.006} & \multirow[t]{8}{*}{287} & 0.477 & 0.008 & 3.8 & \multirow[t]{19}{*}{0.012} & \multirow[t]{6}{*}{281} & 0.415 & 0.019 & 29.0 \\
\hline & & 0.376 & 0.010 & 5.1 & & & 0.431 & 0.020 & 34.0 \\
\hline & & 0.421 & 0.012 & 8.8 & & & 0.419 & 0.021 & 37.1 \\
\hline & & 0.419 & 0.013 & 12.7 & & & 0.420 & 0.022 & 42.3 \\
\hline & & 0.364 & 0.015 & 16.0 & & & 0.407 & 0.023 & 48.8 \\
\hline & & 0.414 & 0.016 & 22.2 & & & 0.423 & 0.022 & 44.3 \\
\hline & & 0.443 & 0.014 & 15.1 & & 279 & 0.438 & 0.030 & 88.1 \\
\hline & & 0.453 & 0.011 & 7.8 & & 281 & 0.463 & 0.025 & 60.0 \\
\hline & \multirow[t]{5}{*}{289} & 0.261 & 0.013 & 5.3 & & & 0.443 & 0.027 & 65.2 \\
\hline & & 0.407 & 0.010 & 7.1 & & & 0.454 & 0.027 & 64.9 \\
\hline & & 0.464 & 0.009 & 6.6 & & & 0.461 & 0.028 & 73.3 \\
\hline & & 0.391 & 0.016 & 20.7 & & & 0.437 & 0.031 & 88.2 \\
\hline & & 0.335 & 0.014 & 17.0 & & & 0.464 & 0.032 & 95.7 \\
\hline & \multirow[t]{6}{*}{290} & 0.376 & 0.011 & 11.3 & & & 0.455 & 0.033 & 104.2 \\
\hline & & 0.361 & 0.016 & 19 & & & 0.422 & 0.023 & 45.4 \\
\hline & & 0.403 & 0.020 & 21.8 & & 283 & 0.367 & 0.019 & 27.3 \\
\hline & & 0.288 & 0.014 & 11.9 & & 282 & 0.270 & 0.028 & 56.4 \\
\hline & & 0.370 & 0.009 & 6.2 & & & 0.276 & 0.026 & 48.1 \\
\hline & & 0.343 & 0.013 & 13.2 & & & 0.279 & 0.024 & 41.0 \\
\hline \multirow[t]{15}{*}{0.012} & \multirow[t]{3}{*}{281} & 0.441 & 0.022 & 41.5 & & 283 & 0.352 & 0.020 & 32.9 \\
\hline & & 0.375 & 0.025 & 51.4 & & & 0.372 & 0.023 & 42.5 \\
\hline & & 0.410 & 0.030 & 77.7 & & & 0.335 & & 42.9 \\
\hline & \multirow[t]{8}{*}{280} & 0.463 & 0.033 & 96.7 & & & 0.276 & 0.025 & 46.7 \\
\hline & & 0.465 & 0.030 & 81.1 & & & 0.354 & 0.023 & 43.1 \\
\hline & & 0.448 & 0.032 & 88.7 & & & 0.328 & 0.026 & 54.3 \\
\hline & & 0.472 & 0.034 & 104.8 & & & 0.320 & 0.030 & 57.8 \\
\hline & & 0.446 & 0.027 & 62.3 & & & 0.295 & 0.031 & 62.6 \\
\hline & & 0.468 & 0.028 & 71.9 & & & 0.293 & & 66.9 \\
\hline & & 0.420 & 0.029 & 75.1 & & & 0.307 & & 72.2 \\
\hline & & 0.470 & 0.033 & 103.4 & 0.020 & 286 & 0.313 & 0.015 & 12.1 \\
\hline & \multirow[t]{4}{*}{281} & 0.483 & 0.026 & 61.8 & & & 0.235 & 0.016 & 12.5 \\
\hline & & 0.423 & 0.029 & 70.0 & & & 0.265 & 0.0118 & 18.7 \\
\hline & & 0.434 & 0.017 & 25.0 & & & 0.213 & 0.020 & 24.2 \\
\hline & & 0.470 & 0.018 & 27.5 & & & 0.313 & 0.019 & 29.2 \\
\hline
\end{tabular}


Table. 5.4: 2nd page

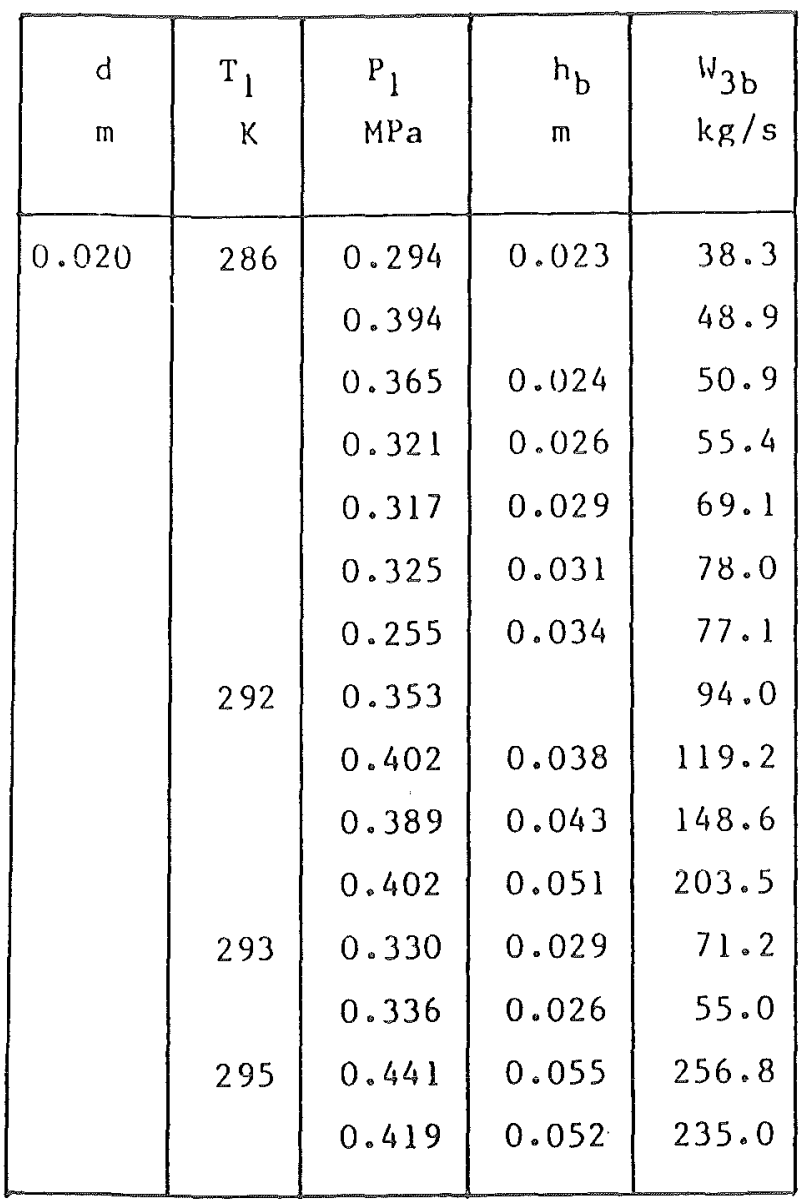

\begin{tabular}{|c|c|c|c|c|}
\hline $\begin{array}{l}\mathrm{d} \\
\mathrm{m}\end{array}$ & $\begin{array}{r}\mathrm{T}_{1} \\
\mathrm{~K}\end{array}$ & $\begin{array}{l}\mathrm{P}_{1} \\
\mathrm{MPa}\end{array}$ & $\begin{array}{l}h_{b} \\
m\end{array}$ & $\begin{array}{l}\mathrm{W}_{3 \mathrm{~b}} \\
10^{-3} \mathrm{~kg} / \mathrm{s}\end{array}$ \\
\hline 0.020 & 295 & $\begin{array}{l}0.317 \\
0.336 \\
0.394 \\
0.389 \\
0.420 \\
0.449 \\
0.449 \\
0.350 \\
0.350 \\
0.340 \\
0.357 \\
0.385 \\
0.365 \\
0.374\end{array}$ & $\begin{array}{l}0.027 \\
0.018 \\
0.027 \\
0.026 \\
0.027 \\
0.028 \\
0.028 \\
0.027 \\
0.027 \\
0.029 \\
0.027 \\
0.024 \\
0.024 \\
0.026\end{array}$ & $\begin{array}{l}60.3 \\
24.3 \\
58.2 \\
58.2 \\
69.5 \\
76.1 \\
76.1 \\
65.4 \\
66.1 \\
59.1\end{array}$ \\
\hline
\end{tabular}


Table 5.5: Beginning of gas entrainment in horizontal branches; $v_{2 g}=0, v_{21}=0$

\begin{tabular}{|c|c|c|c|c|}
\hline $\begin{array}{l}d \\
m\end{array}$ & $\begin{array}{c}\mathrm{T}_{1} \\
\mathrm{~K}\end{array}$ & $\begin{array}{l}\mathrm{P}_{1} \\
\mathrm{MPa}\end{array}$ & $h_{b}$ & $\begin{array}{l}\mathrm{w}_{3 \mathrm{~b}} \\
\mathrm{~kg} / \mathrm{s}\end{array}$ \\
\hline \multirow[t]{26}{*}{0.006} & \multirow[t]{7}{*}{288} & 0.423 & 0.020 & 0.352 \\
\hline & & 0.365 & 0.023 & 0.407 \\
\hline & & 0.333 & 0.022 & 0.366 \\
\hline & & 0.480 & 0.025 & 0.481 \\
\hline & & 0.394 & 0.021 & 0.424 \\
\hline & & 0.406 & & 0.340 \\
\hline & & 0.411 & 0.020 & 0.295 \\
\hline & \multirow[t]{10}{*}{292} & 0.304 & 0.021 & 0.406 \\
\hline & & 0.307 & & 0.344 \\
\hline & & 0.365 & 0.023 & 0.451 \\
\hline & & 0.298 & 0.021 & 0.348 \\
\hline & & 0.252 & 0.019 & 0.255 \\
\hline & & 0.358 & 0.020 & 0.299 \\
\hline & & 0.420 & 0.019 & 0.271 \\
\hline & & 0.395 & 0.017 & 0.194 \\
\hline & & 0.285 & 0.021 & 0.381 \\
\hline & & 0.318 & 0.022 & 0.402 \\
\hline & \multirow[t]{4}{*}{293} & 0.423 & 0.017 & 0.183 \\
\hline & & 0.298 & 0.016 & 0.247 \\
\hline & & 0.340 & 0.019 & 0.310 \\
\hline & & 0.449 & 0.017 & 0.188 \\
\hline & \multirow[t]{3}{*}{294} & 0.365 & 0.013 & 0.121 \\
\hline & & 0.482 & 0.018 & 0.330 \\
\hline & & 0.382 & 0.020 & \\
\hline & 289 & 0.482 & 0.013 & 0.126 \\
\hline & 290 & 0.443 & 0.018 & 0.225 \\
\hline \multirow[t]{6}{*}{0.012} & 281 & 0.433 & 0.022 & 0.483 \\
\hline & \multirow[t]{3}{*}{282} & 0.414 & & 0.794 \\
\hline & & 0.438 & 0.024 & 0.624 \\
\hline & & 0.408 & 0.025 & 0.698 \\
\hline & \multirow[t]{2}{*}{285} & 0.315 & 0.021 & 0.414 \\
\hline & & 0.316 & & 0.369 \\
\hline
\end{tabular}

\begin{tabular}{|c|c|c|c|c|}
\hline $\begin{array}{l}\mathrm{d} \\
\mathrm{m}\end{array}$ & $\begin{array}{l}\mathrm{T}_{1} \\
\mathrm{~K}\end{array}$ & $\begin{array}{l}P_{1} \\
M P_{a}\end{array}$ & $\begin{array}{l}h_{b} \\
m\end{array}$ & $\begin{array}{l}\mathrm{w}_{3 \mathrm{~b}} \\
\mathrm{~kg} / \mathrm{s}\end{array}$ \\
\hline \multirow[t]{21}{*}{0.012} & 285 & 0.294 & 0.023 & 0.618 \\
\hline & & 0.316 & & 0.594 \\
\hline & & 0.309 & 0.020 & 0.415 \\
\hline & & 0.274 & & 0.472 \\
\hline & 288 & 0.409 & 0.025 & 0.603 \\
\hline & & 0.406 & 0.028 & 0.797 \\
\hline & 296 & 0.358 & 0.024 & 0.568 \\
\hline & & 0.360 & 0.026 & 0.713 \\
\hline & & 0.363 & 0.031 & 1.064 \\
\hline & & 0.360 & 0.032 & 1.275 \\
\hline & & 0.370 & 0.036 & 1.843 \\
\hline & 283 & 0.317 & 0.019 & 0.349 \\
\hline & & 0.316 & 0.021 & 0.457 \\
\hline & & 0.315 & 0.024 & 0.640 \\
\hline & & 0.384 & 0.025 & 0.816 \\
\hline & & 0.385 & 0.028 & 1.027 \\
\hline & & 0.383 & 0.029 & 1.252 \\
\hline & 287 & 0.374 & 0.031 & 0.913 \\
\hline & & 0.375 & 0.035 & 1.288 \\
\hline & & 0.394 & 0.036 & 1.526 \\
\hline & & 0.356 & 0.033 & 1.026 \\
\hline \multirow[t]{11}{*}{0.020} & 286 & 0.418 & 0.016 & 0.166 \\
\hline & & 0.405 & 0.022 & 0.501 \\
\hline & & 0.465 & & 0.560 \\
\hline & & 0.422 & & 0.425 \\
\hline & & 0.327 & 0.021 & 0.398 \\
\hline & & 0.306 & 0.026 & 0.716 \\
\hline & & 0.327 & & 0.701 \\
\hline & & 0.419 & 0.025 & 0.622 \\
\hline & & 0.477 & 0.026 & 0.633 \\
\hline & 292 & 0.376 & 0.019 & 0.266 \\
\hline & & 0.412 & $(0.1) 20$ & 0.373 \\
\hline
\end{tabular}


Table 5.5: (2nd page)

\begin{tabular}{|c|c|c|c|c|}
\hline $\begin{array}{l}d \\
\text { m }\end{array}$ & $\begin{array}{l}\Gamma_{1} \\
k\end{array}$ & $\begin{array}{l}\mathrm{P}_{1} \\
\mathrm{MPa}\end{array}$ & ${ }_{m}^{h_{b}}$ & $\begin{array}{l}W_{3 b} \\
\mathrm{~kg} / \mathrm{s}\end{array}$ \\
\hline 0.020 & $\begin{array}{l}293 \\
292\end{array}$ & $\begin{array}{l}0.311 \\
0.325 \\
0.306 \\
0.384 \\
0.394 \\
0.404 \\
0.338 \\
0.453 \\
0.318 \\
0.314\end{array}$ & $\begin{array}{l}0.022 \\
0.026 \\
0.024 \\
0.033 \\
0.034 \\
0.051 \\
0.026 \\
0.028 \\
0.029 \\
0.023\end{array}$ & $\begin{array}{l}0.571 \\
0.635 \\
0.472 \\
1.396 \\
1.779 \\
3.809 \\
0.699 \\
0.661 \\
0.836 \\
0.639\end{array}$ \\
\hline
\end{tabular}

\begin{tabular}{|c|c|c|c|c|}
\hline $\begin{array}{l}\mathrm{d} \\
\mathrm{m}\end{array}$ & $\begin{array}{l}\Gamma_{1} \\
k\end{array}$ & $\begin{array}{l}P_{1} \\
M P_{a}\end{array}$ & $n_{b}$ & $\begin{array}{l}W_{3 b} \\
\mathrm{~kg} / \mathrm{s}\end{array}$ \\
\hline 0.020 & $\begin{array}{l}290 \\
294\end{array}$ & $\begin{array}{l}0.368 \\
0.309 \\
0.387 \\
0.394 \\
0.414 \\
0.407 \\
0.406 \\
0.404 \\
0.386 \\
0.445\end{array}$ & $\begin{array}{l}0.022 \\
0.030 \\
0.024 \\
0.022 \\
0.023 \\
0.025 \\
0.023 \\
0.026 \\
0.030 \\
0.022\end{array}$ & $\begin{array}{l}0.489 \\
1.063 \\
0.479 \\
0.496 \\
0.539 \\
0.545 \\
0.475 \\
0.711 \\
0.811 \\
0.463\end{array}$ \\
\hline
\end{tabular}


Table 5.6: Beginning of liquid entrainment in upward branches; $v_{2 \mu}>0, v_{21}=0$

\begin{tabular}{|c|c|c|c|c|c|}
\hline $\begin{array}{l}d \\
m\end{array}$ & $\begin{array}{l}\mathrm{T}_{1} \\
\mathrm{~K}\end{array}$ & $\begin{array}{l}\mathrm{P}_{1} \\
\mathrm{MPa}\end{array}$ & $\begin{array}{l}\mathrm{h}_{\mathrm{b}} \\
\mathrm{m}\end{array}$ & $\begin{array}{c}W_{3 b} \\
10^{-3}\end{array}$ & $\begin{array}{l}W_{2 g} \\
\mathrm{~kg} / \mathrm{s}\end{array}$ \\
\hline 0.012 & $\begin{array}{l}289 \\
290 \\
291\end{array}$ & $\begin{array}{l}0.410 \\
0.481 \\
0.440 \\
0.412 \\
0.395 \\
0.368 \\
0.452 \\
0.470 \\
0.482 \\
0.346 \\
0.412 \\
0.213 \\
0.438 \\
0.458 \\
0.463\end{array}$ & $\begin{array}{l}0.049 \\
0.056 \\
0.058 \\
0.066 \\
0.046 \\
0.054 \\
0.068 \\
0.044\end{array}$ & $\begin{array}{r}88.7 \\
100.8 \\
83.5 \\
81.8 \\
69.4 \\
56.6 \\
38.9 \\
90.3 \\
48.0 \\
75.1 \\
53.4 \\
89.2 \\
43.8 \\
73.0 \\
26.1\end{array}$ & $\begin{array}{r}11.6 \\
22.0 \\
17.7 \\
14.1 \\
15.7 \\
12.1 \\
13.6 \\
11.7 \\
3.2 \\
11.6 \\
6.4 \\
8.5 \\
4.5 \\
11.6 \\
20.6 \\
21.5\end{array}$ \\
\hline
\end{tabular}

\begin{tabular}{|c|c|c|c|c|c|}
\hline $\begin{array}{l}\mathrm{d} \\
\mathrm{m}\end{array}$ & $\mid \begin{array}{l}\mathrm{T}_{1} \\
\mathrm{k}\end{array}$ & $\begin{array}{l}P_{1} \\
\mathrm{NPa}\end{array}$ & $\begin{array}{l}\mathrm{h}_{\mathrm{b}} \\
\mathrm{m}\end{array}$ & $\begin{array}{c}w_{3 b} \\
10^{-3}\end{array}$ & $\begin{array}{l}W_{2 g} \\
g / s\end{array}$ \\
\hline \multirow[t]{14}{*}{0.012} & 280 & 0.298 & 0.044 & 36.2 & 21.6 \\
\hline & & 0.405 & 0.046 & 38.6 & 18.1 \\
\hline & & 0.460 & & 39.8 & 15.7 \\
\hline & & 0.288 & & 35.8 & 19.6 \\
\hline & & 0.386 & 0.047 & 60.7 & 30.8 \\
\hline & & 0.363 & & 59.3 & 32.1 \\
\hline & & 0.353 & 0.046 & 58.3 & 33.2 \\
\hline & & 0.385 & & 66.7 & \\
\hline & & 0.415 & 0.048 & 73.0 & 35.8 \\
\hline & & 0.394 & 0.061 & 70.6 & 36.3 \\
\hline & & 0.353 & & 58.9 & 35.7 \\
\hline & & 0.403 & 0.051 & 70.4 & 36.3 \\
\hline & 281 & 0.340 & & 60.9 & 27.0 \\
\hline & & 0.320 & 0.057 & 48.7 & 25.4 \\
\hline \multirow[t]{2}{*}{0.020} & 279 & 0.404 & 0.049 & 102.2 & 17.7 \\
\hline & & 0.394 & 0.060 & 94.4 & 37.2 \\
\hline
\end{tabular}


Table 5.7: Beginning of liquid entrainment in upward branches; $v_{2 g}=0, v_{21}>0$

\begin{tabular}{|c|c|c|c|c|c|}
\hline $\begin{array}{l}\mathrm{d} \\
\mathrm{m}\end{array}$ & $\begin{array}{l}I_{1} \\
K\end{array}$ & $\begin{array}{l}\mathrm{P}_{1} \\
\mathrm{MPa}\end{array}$ & $\begin{array}{l}\mathrm{h}_{\mathrm{b}} \\
\mathrm{m}\end{array}$ & $\begin{array}{c}{ }^{\mathrm{W}} \mathrm{b} \\
10^{-3} \mathrm{~kg} / \mathrm{s}\end{array}$ & $\begin{array}{c}\mathrm{W} \\
21 \\
\mathrm{~kg} / \mathrm{s}\end{array}$ \\
\hline 0.012 & 281 & $\begin{array}{l}0.372 \\
0.459 \\
0.451 \\
0.401 \\
0.396 \\
0.364\end{array}$ & $\begin{array}{l}0.071 \\
0.073 \\
0.071 \\
0.076 \\
0.070 \\
0.081 \\
0.071 \\
0.073\end{array}$ & 92.0 & $\begin{array}{l}2.08 \\
0.75\end{array}$ \\
\hline
\end{tabular}

\begin{tabular}{|c|c|c|c|c|c|}
\hline $\begin{array}{l}\mathrm{d} \\
\mathrm{m}\end{array}$ & $\begin{array}{l}I_{1} \\
K\end{array}$ & $\begin{array}{l}\mathrm{P}_{1} \\
\mathrm{MPa}\end{array}$ & $\begin{array}{l}\mathrm{h}_{\mathrm{b}} \\
\mathrm{m}\end{array}$ & $\begin{array}{c}\mathrm{W}^{3 \mathrm{~b}} \\
1 \overline{0}^{-3} \mathrm{~kg} / \mathrm{s}\end{array}$ & $\begin{array}{l}\mathrm{W} \\
21 \\
\mathrm{~kg} / \mathrm{s}\end{array}$ \\
\hline 0.020 & 280 & $\begin{array}{l}0.327 \\
0.410 \\
0.412 \\
0.347 \\
0.404 \\
0.424 \\
0.366 \\
0.394\end{array}$ & $\begin{array}{l}0.072 \\
0.049 \\
0.074 \\
0.073 \\
0.060\end{array}$ & $\begin{array}{r}85.0 \\
131.0 \\
108.8 \\
84.1 \\
102.2 \\
114.9 \\
98.1 \\
94.4\end{array}$ & $\begin{array}{l}2.07 \\
2.16 \\
2.15 \\
0.88 \\
2.05 \\
1.99 \\
2.05 \\
2.07\end{array}$ \\
\hline
\end{tabular}


Table 5.8: Beginning of liquid entrainment in horizontal branches, $v_{2 g}>0 ; v_{2 \downarrow}=0$

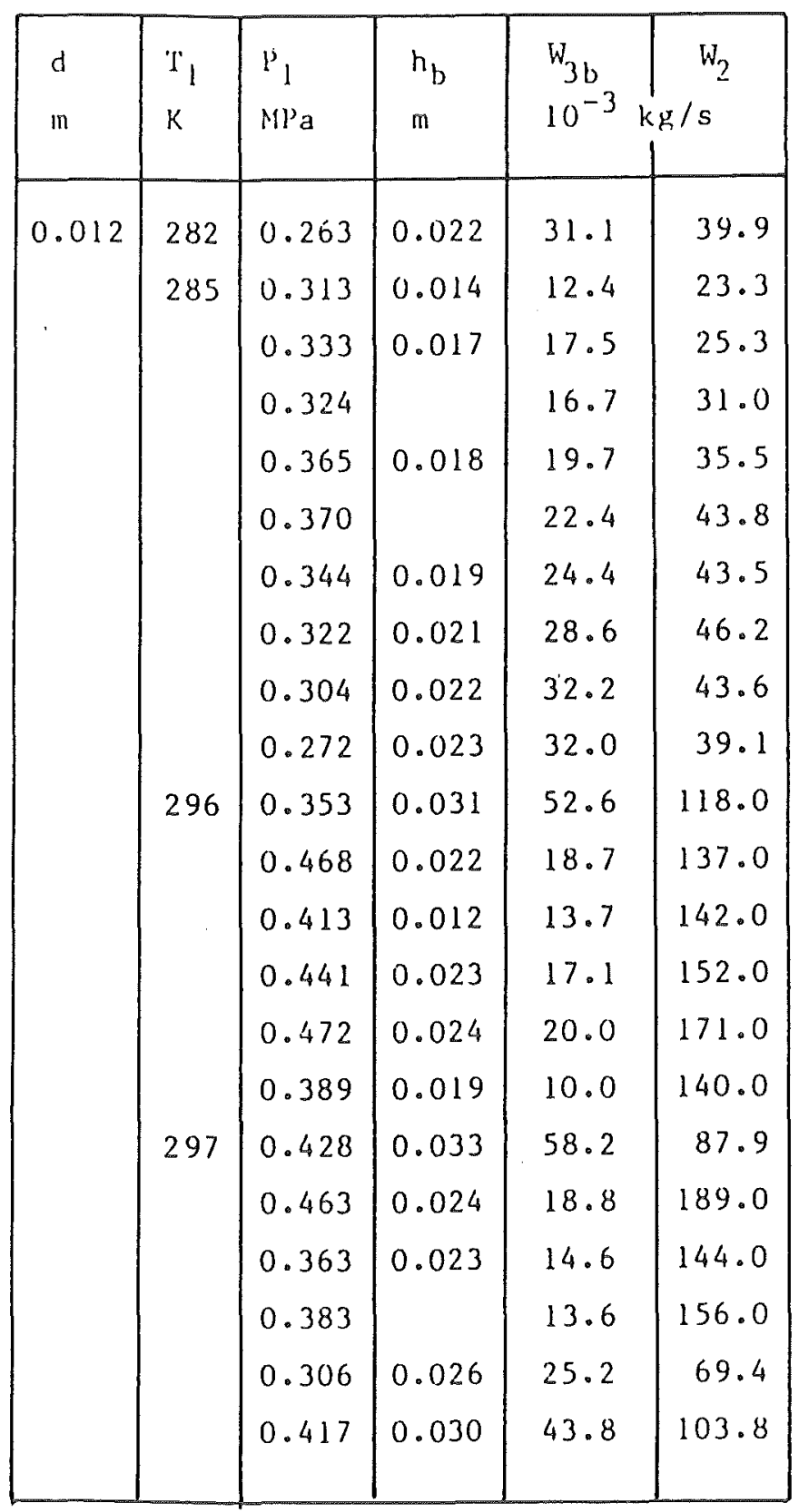

\begin{tabular}{|c|c|c|c|c|c|}
\hline $\begin{array}{l}d \\
m\end{array}$ & $\begin{array}{l}\mathrm{T}_{1} \\
\mathrm{~K}\end{array}$ & $\begin{array}{l}\mathrm{P}_{1} \\
\mathrm{MPa}\end{array}$ & $\begin{array}{l}h_{b} \\
m\end{array}$ & $\begin{array}{l}w_{3 b} \\
10^{-3}\end{array}$ & $\begin{array}{r}w_{2} \\
k g / s\end{array}$ \\
\hline \multirow[t]{7}{*}{0.012} & 297 & 0.338 & 0.029 & 57.4 & 86.5 \\
\hline & & 0.418 & 0.024 & 34.4 & 105.3 \\
\hline & & 0.380 & & 34.0 & 115.5 \\
\hline & & 0.379 & 0.016 & 15.3 & 106.7 \\
\hline & & 0.315 & 0.015 & 12.3 & 89.2 \\
\hline & & 0.304 & 0.016 & 12.4 & 71.9 \\
\hline & 298 & 0.412 & 0.028 & 69.2 & 100 \\
\hline \multirow[t]{15}{*}{0.020} & 293 & 0.317 & 0.027 & 60.3 & 23.4 \\
\hline & & 0.305 & 0.024 & 31.4 & 47.6 \\
\hline & & 0.389 & 0.019 & 18.5 & 61.1 \\
\hline & & 0.336 & 0.018 & 24.3 & 52.7 \\
\hline & & 0.296 & 0.026 & 41.9 & 36.1 \\
\hline & 294 & 0.314 & 0.041 & 95.4 & 66.9 \\
\hline & & 0.390 & 0.036 & 67.7 & 84.6 \\
\hline & & 0.430 & 0.032 & 41.0 & 107.8 \\
\hline & & 0.424 & 0.027 & 25.7 & 112.7 \\
\hline & & 0.463 & 0.024 & 17.3 & 132.7 \\
\hline & & 0.443 & 0.019 & 13.1 & 145.2 \\
\hline & 295 & 0.453 & & 15.0 & 173.1 \\
\hline & & 0.397 & 0.027 & 23.0 & 151.1 \\
\hline & & 0.389 & & 25.0 & 149.0 \\
\hline & & 0.355 & 0.030 & 27.4 & 118.6 \\
\hline
\end{tabular}


Table 5.9: Beginning of liquid entrainment in horizontal branches, $v_{2 g}=0, v_{21}>0$

\begin{tabular}{|c|c|c|c|c|c|}
\hline $\begin{array}{l}d \\
\mathrm{~m}\end{array}$ & $\begin{array}{l}\mathrm{T}_{1} \\
\mathrm{~K}\end{array}$ & $\begin{array}{l}\mathrm{P}_{1} \\
\mathrm{MPa}\end{array}$ & $h_{b}$ & $\begin{array}{c}W_{3 \mathrm{~b}} \\
10^{-3} \mathrm{~kg} / \mathrm{s}\end{array}$ & $W_{21}$ \\
\hline \multirow[t]{19}{*}{0.012} & 283 & 0.303 & 0.017 & 17.9 & 0.663 \\
\hline & & 0.367 & 0.019 & 27.3 & 0.814 \\
\hline & 285 & 0.352 & 0.021 & 32.9 & 1.147 \\
\hline & & 0.372 & 0.023 & 42.5 & 1.118 \\
\hline & & 0.320 & 0.030 & 57.8 & 1.665 \\
\hline & & 0.295 & 0.031 & 62.6 & 1.825 \\
\hline & & 0.293 & & 66.9 & 1.890 \\
\hline & 295 & 0.396 & 0.026 & 47.1 & 5.00 \\
\hline & & 0.445 & & 46.4 & 5.38 \\
\hline & & 0.345 & & 33.5 & 6.33 \\
\hline & 296 & 0.389 & 0.019 & 25.2 & 5.91 \\
\hline & & 0.419 & 0.021 & 23.3 & 6.37 \\
\hline & & 0.433 & 0.019 & 19.6 & 6.29 \\
\hline & & 0.394 & 0.024 & 25.5 & 6.75 \\
\hline & & 0.330 & 0.021 & 28.1 & 7.35 \\
\hline & & 0.335 & & 28.2 & 7.80 \\
\hline & & & 0.023 & 24.3 & 8.17 \\
\hline & & 0.306 & 0.024 & 36.1 & 8.44 \\
\hline & 297 & 0.485 & 0.028 & 59.5 & 3.30 \\
\hline \multirow[t]{2}{*}{0.020} & 293 & 0.345 & 0.027 & 42.8 & 3.08 \\
\hline & & 0.394 & & 58.2 & 2.92 \\
\hline
\end{tabular}

\begin{tabular}{|c|c|c|c|c|c|}
\hline $\begin{array}{l}\mathrm{d} \\
\mathrm{m}\end{array}$ & $\begin{array}{l}\mathrm{T}_{1} \\
\mathrm{~K}\end{array}$ & $\begin{array}{l}\mathrm{P}_{1} \\
\mathrm{MPa}\end{array}$ & $\begin{array}{l}\mathrm{h}_{\mathrm{b}} \\
\mathrm{m}\end{array}$ & $\begin{array}{c}\mathrm{W}_{3 \mathrm{~b}} \\
10^{-3} \mathrm{~kg} / \mathrm{s}\end{array}$ & $\begin{array}{l}\mathrm{W}_{21} \\
\mathrm{~kg} / \mathrm{s}\end{array}$ \\
\hline \multirow[t]{21}{*}{0.020} & 293 & 0.420 & 0.027 & 69.5 & 2.85 \\
\hline & & 0.449 & 0.028 & 76.1 & 2.72 \\
\hline & & & & & 2.46 \\
\hline & 294 & 0.409 & 0.037 & 75.0 & 3.18 \\
\hline & & 0.433 & 0.035 & 87.8 & 3.07 \\
\hline & & 0.443 & 0.033 & 64.9 & 2.98 \\
\hline & & 0.442 & 0.042 & 110.9 & 3.24 \\
\hline & 290 & 0.350 & 0.027 & 65.4 & 1.86 \\
\hline & & & & 66.1 & \\
\hline & & 0.340 & 0.029 & 59.1 & 2.44 \\
\hline & & 0.357 & 0.027 & & 2.29 \\
\hline & & 0.385 & 0.024 & 42.3 & 2.61 \\
\hline & & 0.365 & & 41.8 & 3.18 \\
\hline & & 0.467 & 0.028 & & 4.31 \\
\hline & & 0.350 & 0.026 & & 4.90 \\
\hline & 295 & 0.414 & & 46.2 & 7.31 \\
\hline & & 0.374 & & 46.7 & 6.75 \\
\hline & & 0.406 & 0.029 & 35.9 & 6.45 \\
\hline & 294 & 0.338 & 0.030 & 47.6 & 7.96 \\
\hline & & 0.365 & 0.028 & & 8.72 \\
\hline & & 0.459 & 0.026 & 26.7 & 9.08 \\
\hline
\end{tabular}


Table 5.10: Beginning of gas entrainment in horizontal branches; $v_{2 g}>0, v_{21}=0$

\begin{tabular}{|c|c|c|c|c|c|}
\hline $\mathrm{d}$ & $\begin{array}{c}\mathrm{T}_{1} \\
\mathrm{~K}\end{array}$ & $\begin{array}{c}\mathrm{P}_{1} \\
\mathrm{MPa}\end{array}$ & $\begin{array}{c}\mathrm{h}_{\mathrm{b}} \\
\mathrm{m}\end{array}$ & $\begin{array}{c}\mathrm{W}_{3 \mathrm{~b}} \\
\mathrm{~kg} / \mathrm{s}\end{array}$ & $\begin{array}{c}\mathrm{W}_{2 \mathrm{~g}} \\
10^{-3} \mathrm{~kg} / \mathrm{s}\end{array}$ \\
\hline \multirow{3}{*}{0.012} & 289 & 0.402 & 0.025 & 0.467 & 79.8 \\
& & 0.404 & 0.023 & 0.337 & 79.9 \\
& & 0.385 & 0.025 & 0.413 & 76.8 \\
& & 0.373 & 0.026 & 0.444 & 71.1 \\
& & 0.318 & 0.023 & 0.408 & 60.6 \\
& & 0.289 & 0.022 & 0.287 & 54.0 \\
& \multirow{3}{*}{296} & 0.384 & & 0.338 & 75.0 \\
& & 0.235 & & 0.350 & 63.1 \\
& & 0.368 & 0.021 & 0.403 & 89.6 \\
& & 0.016 & 0.222 & 99.3 \\
& 292 & 0.366 & & 0.223 & 108 \\
\hline
\end{tabular}

\begin{tabular}{|l|l|l|l|l|l|}
\hline $\mathrm{d}$ & $\begin{array}{l}\mathrm{T}_{1} \\
\mathrm{~m}\end{array}$ & $\begin{array}{l}\mathrm{P}_{1} \\
\mathrm{MPa}\end{array}$ & $\begin{array}{l}\mathrm{h}_{\mathrm{b}} \\
\mathrm{m}\end{array}$ & $\begin{array}{l}\mathrm{W}_{3 \mathrm{~b}} \\
\mathrm{~kg} / \mathrm{s}\end{array}$ & $\begin{array}{c}\mathrm{W}_{2 \mathrm{~g}} \\
10^{-3} \mathrm{~kg} / \mathrm{s}\end{array}$ \\
\hline 0.020 & 292 & 0.407 & 0.019 & 0.296 & 49.8 \\
& & 0.372 & & 0.301 & 48.9 \\
& & 0.387 & 0.022 & 0.382 & 58.2 \\
& & 0.024 & 0.479 & 64.7 \\
& & 0.394 & 0.022 & 0.496 & 69.8 \\
& & 0.414 & 0.023 & 0.539 & 72.6 \\
& 0.407 & 0.025 & 0.545 & 76.7 \\
& 0.406 & 0.023 & 0.475 & 76.3 \\
& 0.404 & 0.026 & 0.711 & \\
& \multirow{3}{*}{294} & 0.463 & 0.023 & 0.439 & 91.0 \\
& 0.445 & 0.022 & 0463 & 88.7 \\
& & & & \\
\hline
\end{tabular}


Table 5.11: Beginning of gas entrainment in horizontal branches; $v_{2 g}=0, v_{21}>0$

\begin{tabular}{|l|l|l|l|l|l|}
\hline$d$ & $T_{1}$ & $P_{1}$ & $h_{b}$ & $\begin{array}{l}W_{3 b} \\
\mathrm{mg} / \mathrm{s}\end{array}$ & $\begin{array}{l}W_{21} \\
\mathrm{~kg} / \mathrm{s}\end{array}$ \\
\hline \multirow{3}{*}{0.012} & \multirow{2}{*}{287} & 0.281 & 0.032 & 0.742 & 2.80 \\
& & 0.384 & 0.036 & 1.050 & 3.02 \\
& & 0.385 & 0.035 & 1.222 & 3.11 \\
& \multirow{5}{*}{295} & 0.347 & 0.026 & 0.415 & 4.59 \\
& & 0.288 & 0.032 & 0.858 & 4.77 \\
& & 0.288 & 0.031 & 0.800 & 7.46 \\
& & 0.365 & 0.020 & 0.205 & 7.00 \\
& & 0.377 & & 0.194 & 5.63 \\
& & 0.355 & 0.018 & 0.180 & 8.52 \\
& & 0.365 & 0.019 & 0.200 & 7.51 \\
& & 0.355 & 0.026 & 0.493 & 9.50 \\
& & 0.394 & 0.025 & 0.232 & 10.92 \\
& & 0.343 & 0.019 & 0.240 & 2.54 \\
\hline
\end{tabular}

\begin{tabular}{|c|c|c|c|c|c|}
\hline $\begin{array}{l}d \\
\text { in }\end{array}$ & $\begin{array}{l}\mathrm{T}_{1} \\
\mathrm{k}\end{array}$ & $\begin{array}{l}\mathrm{P}_{1} \\
\mathrm{MPa}\end{array}$ & $\begin{array}{l}h_{b} \\
m\end{array}$ & $\begin{array}{l}\mathrm{w}_{3 \mathrm{~b}} \\
\mathrm{~kg} / \mathrm{s}\end{array}$ & $\begin{array}{l}\mathrm{W}_{21} \\
\mathrm{ks} / \mathrm{s}\end{array}$ \\
\hline 0.020 & 292 & $\begin{array}{l}0.343 \\
0.338 \\
0.318 \\
0.314 \\
0.318 \\
0.320 \\
0.386 \\
0.382 \\
0.387 \\
0.372 \\
0.374\end{array}$ & $\begin{array}{l}0.023 \\
0.026 \\
0.028 \\
0.029 \\
0.023 \\
0.026 \\
0.025 \\
0.030 \\
0.029 \\
0.025 \\
0.034 \\
0.029\end{array}$ & $\begin{array}{l}0.431 \\
0.699 \\
0.661 \\
0.836 \\
0.639 \\
0.408 \\
0.394 \\
0.811 \\
0.625 \\
0.408 \\
0.786 \\
0.527\end{array}$ & $\begin{array}{l}2.64 \\
2.69 \\
3.87 \\
2.95 \\
3.49 \\
4.18 \\
4.99 \\
3.94 \\
3.91 \\
3.69 \\
5.50 \\
6.51\end{array}$ \\
\hline
\end{tabular}


Table 5.12: Gas entrainment in downward branches.

\begin{tabular}{|c|c|c|c|c|c|c|c|c|c|c|c|c|c|}
\hline $\begin{array}{l}\mathrm{d} \\
\mathrm{m}\end{array}$ & $\begin{array}{l}T_{1} \\
K\end{array}$ & $\begin{array}{l}\mathrm{P}_{1} \\
\mathrm{MPa}\end{array}$ & $\begin{array}{c}\Delta \mathrm{P}_{\mathrm{I}-34} \\
\mathrm{MPa}\end{array}$ & $\begin{array}{l}\mathrm{h} \\
\mathrm{m}\end{array}$ & $\begin{array}{c}\mathrm{w}_{3 \mathrm{~g}} \\
10^{-3} \mathrm{~kg} / \mathrm{s}\end{array}$ & $\begin{array}{l}\mathrm{W}_{31} \\
\mathrm{~kg} / \mathrm{s}\end{array}$ & $\begin{array}{l}\mathrm{d} \\
\mathrm{m}\end{array}$ & $\begin{array}{l}\mathrm{T}_{1} \\
\mathrm{~K}\end{array}$ & $\begin{array}{l}\mathrm{P}_{1} \\
\mathrm{MPa}\end{array}$ & $\begin{array}{c}\Delta \mathrm{P}_{1-34} \\
\mathrm{MPa}\end{array}$ & $\begin{array}{l}\mathrm{h} \\
\mathrm{m}\end{array}$ & $\begin{array}{c}\mathrm{W}_{3 \mathrm{~g}} \\
10^{-3} \mathrm{~kg} / \mathrm{s}\end{array}$ & $\begin{array}{l}\mathrm{H}_{31} \\
\mathrm{~kg} / \mathrm{s}\end{array}$ \\
\hline \multirow[t]{7}{*}{0.006} & 287 & 0.364 & 0.225 & 0.017 & 6.00 & 0.175 & 0.008 & 294 & 0.521 & 0.122 & 0.017 & 6.81 & 0.225 \\
\hline & & 0.384 & 0.240 & 0.020 & 5.83 & 0.210 & & & 0.493 & 0.220 & 0.022 & 7.05 & 0.326 \\
\hline & & 0.546 & 0.340 & 0.016 & 6.36 & 0.203 & & & 0.482 & 0.235 & 0.040 & 5.21 & 0.491 \\
\hline & & 0.467 & 0.250 & 0.015 & 7.20 & 0.145 & 0.012 & 296 & & 0.024 & 0.030 & 5.63 & 0.333 \\
\hline & & 0.517 & 0.310 & 0.017 & 5.71 & 0.201 & & & 0.443 & 0.120 & 0.052 & 6.34 & 0.854 \\
\hline & & 0.445 & 0.260 & 0.014 & 6.31 & 0.157 & & & 0.502 & 0.130 & 0.055 & 6.04 & 0.970 \\
\hline & & 0.520 & 0.145 & 0.018 & 5.76 & 0.144 & & 291 & 0.287 & 0.125 & 0.026 & 9.48 & 0.592 \\
\hline \multirow[t]{10}{*}{0.008} & 295 & 0.482 & 0.199 & 0.039 & 4.66 & 0.434 & & & 0.322 & 0.135 & 0.030 & 9.32 & 0.693 \\
\hline & & 0.492 & 0.122 & 0.020 & 5.98 & 0.284 & & & 0.370 & 0.165 & 0.041 & 9.04 & 0.855 \\
\hline & & 0.521 & 0.119 & 0.017 & 8.66 & 0.262 & & & 0.389 & 0.175 & 0.033 & 11.38 & 0.806 \\
\hline & & 0.531 & 0.066 & 0.026 & 4.06 & 0.260 & & & 0.365 & 0.150 & 0.051 & 6.80 & 0.877 \\
\hline & & 0.480 & 0.200 & 0.023 & 8.87 & 0.348 & & & 0.394 & 0.165 & 0.057 & 6.57 & 0.966 \\
\hline & & 0.536 & 0.135 & 0.020 & 6.33 & 0.222 & & & 0.417 & 0.175 & 0.048 & 9.09 & 0.906 \\
\hline & & 0.460 & 0.190 & 0.023 & 5.28 & 0.398 & & & 0.443 & 0.190 & 0.054 & 8.78 & 1.00 \\
\hline & 294 & 0.463 & 0.255 & 0.045 & 3.64 & 0.534 & & & 0.456 & 0.180 & 0.016 & 8.69 & 1.90 \\
\hline & & 0.541 & 0.250 & 0.038 & 6.56 & 0.474 & & & 0.472 & 0.035 & 0.023 & 8.42 & 0.349 \\
\hline & & 0.482 & 0.060 & 0.021 & 6.33 & 0.199 & & & & & & & \\
\hline
\end{tabular}


Table 5.13: Liquid entrainment in upward branches

\begin{tabular}{|c|c|c|c|c|c|c|c|c|c|c|c|c|c|}
\hline d & $\begin{array}{l}T_{1} \\
X\end{array}$ & $\begin{array}{l}\mathrm{P}_{1} \\
\mathrm{MPa}\end{array}$ & $\begin{array}{l}\Delta \mathrm{P}_{1-34} \\
\mathrm{MPa}\end{array}$ & $\begin{array}{l}\mathrm{h} \\
\mathrm{m}\end{array}$ & ${ }_{10}^{W_{3}}$ & $\begin{array}{l}\mathrm{W}_{31} \\
\mathrm{~g} / \mathrm{s}\end{array}$ & $\begin{array}{l}d \\
\text { m }\end{array}$ & $\begin{array}{l}T_{1} \\
k\end{array}$ & $\begin{array}{l}\mathrm{P}_{1} \\
\mathrm{MPa}\end{array}$ & $\begin{array}{l}\Delta \mathrm{P}_{1-34} \\
\mathrm{MPa}\end{array}$ & $\begin{array}{l}\mathrm{h} \\
\mathrm{m}\end{array}$ & ${ }_{10^{-3}}$ & $w_{31}$ \\
\hline \multirow[t]{32}{*}{0.012} & 290 & 0.403 & 0.130 & 0.064 & 78.8 & 0.53 & 0.012 & 281 & 0.474 & 0.194 & 0.046 & 112.2 & 1.37 \\
\hline & & 0.422 & 0.038 & 0.049 & 51.2 & 0.51 & & & 0.472 & 0.222 & & 114.5 & 1.05 \\
\hline & & 0.433 & 0.060 & 0.056 & 62.3 & 0.52 & & & 0.458 & 0.215 & 0.049 & 110.1 & 0.91 \\
\hline & & 0.435 & 0.070 & 0.062 & 74.5 & 0.20 & & & 0.512 & 0.240 & 0.054 & 122.5 & 2.02 \\
\hline & & 0.462 & 0.113 & & 92.7 & 1.10 & & & 0.463 & 0.218 & 0.056 & 112.1 & 0.44 \\
\hline & & 0.367 & 0.172 & 0.055 & 85.7 & 1.45 & & & 0.487 & 0.228 & 0.061 & 117.1 & 0.54 \\
\hline & & 0.435 & 0.160 & 0.066 & 97.7 & 0.95 & & & 0.228 & 0.096 & 0.041 & 51.6 & 0.95 \\
\hline & & 0.315 & 0.124 & 0.046 & 71.6 & 0.39 & & & 0.338 & 0.159 & 0.044 & 80.8 & 2.13 \\
\hline & & 0.360 & 0.169 & 0.048 & 84.6 & 0.81 & & & 0.469 & 0.220 & 0.046 & 112.2 & 1.98 \\
\hline & & 0.376 & 0.138 & 0.054 & & 1.65 & 0.020 & 292 & 0.482 & 0.024 & 0.071 & 122.6 & 0.89 \\
\hline & 289 & 0.302 & 0.059 & 0.048 & 48.3 & 0.18 & & & 0.492 & 0.015 & 0.072 & 99.3 & 0.38 \\
\hline & & 0.477 & 0.032 & & & 0.41 & & & 0.335 & 0.012 & 0.070 & 71.2 & 0.17 \\
\hline & 285 & 0.453 & 0.133 & 0.069 & 91.3 & 0.38 & & 293 & 0.433 & 0.007 & 0.060 & 70.7 & 0.14 \\
\hline & & 0.418 & 0.196 & 0.073 & 93.1 & 0.03 & & & 0.413 & 0.011 & 0.061 & 85.6 & 0.73 \\
\hline & & 0.432 & 0.088 & 0.043 & 73.6 & 2.68 & & & 0.492 & 0.009 & 0.062 & & 0.56 \\
\hline & 280 & 0.463 & 0.128 & 0.059 & 94.4 & 0.97 & & & 0.340 & 0.014 & 0.069 & & 0.47 \\
\hline & & 0.379 & 0.178 & 0.044 & 86.4 & 1.16 & & & 0.440 & 0.020 & 0.075 & 117.9 & 0.07 \\
\hline & & 0.397 & 0.137 & 0.043 & 86.1 & 0.88 & & 280 & 0.481 & 0.052 & 0.076 & 206.9 & 0.51 \\
\hline & & 0.416 & 0.195 & 0.053 & 96.1 & 1.75 & & & 0.511 & 0.064 & 0.077 & 234.4 & 0.38 \\
\hline & & 0.404 & 0.154 & 0.055 & 89.1 & 1.32 & & & 0.343 & 0.015 & 0.047 & 83.1 & 1.50 \\
\hline & & 0.333 & 0.119 & 0.042 & 71.0 & 1.18 & & & 0.235 & 0.024 & 0.052 & & 1.20 \\
\hline & & 0.406 & 0.152 & & 89.4 & 1.41 & & & 0.404 & 0.028 & 0.056 & 125.4 & 1.38 \\
\hline & & 0.376 & 0.140 & & 82.8 & 2.36 & & & 0.470 & 0.024 & 0.057 & & 2.07 \\
\hline & & 0.423 & 0.199 & 0.036 & 67.6 & 1.84 & & & 0.447 & 0.044 & 0.061 & 186.7 & 1.14 \\
\hline & & 0.394 & 0.185 & 0.032 & 46.2 & 3.52 & & & 0.393 & & 0.065 & 171.8 & 0.58 \\
\hline & & 0.294 & 0.131 & 0.041 & 63.8 & 3.40 & & & 0.461 & 0.056 & 0.066 & 207.8 & 1.27 \\
\hline & & 0.399 & 0.187 & 0.044 & 93.4 & 3.08 & & & 0.374 & 0.039 & 0.072 & 159.4 & 0.60 \\
\hline & & 0.414 & 0.195 & 0.046 & 95.0 & 1.30 & & 282 & 0.451 & 0.018 & 0.049 & 102.7 & 2.98 \\
\hline & & 0.345 & & 0.042 & 67.1 & 3.91 & & & 0.461 & 0.023 & 0.052 & 119.2 & 2.21 \\
\hline & & 0.364 & 0.171 & 0.073 & 85.6 & 0.08 & & & & 0.026 & 0.057 & 126.9 & 1.50 \\
\hline & & 0.340 & 0.160 & 0.042 & 76.3 & 1.54 & & & 0.375 & 0.028 & 0.056 & 118.7 & 2.16 \\
\hline & & 0.308 & 0.146 & 0.041 & 68.4 & 3.60 & & & & & & & \\
\hline
\end{tabular}


Table 5.14: Gas entrainment in horizontal branches

\begin{tabular}{|c|c|c|c|c|c|c|c|c|c|c|c|c|c|}
\hline $\begin{array}{l}d \\
\mathrm{~m}\end{array}$ & $\begin{array}{l}\mathrm{T}_{1} \\
\mathrm{~K}\end{array}$ & $\begin{array}{l}\mathrm{P}_{1} \\
\mathrm{MPa}\end{array}$ & $\underset{\mathrm{MPa}}{\Delta \mathrm{P}^{-34}}$ & $\mathrm{~m}$ & $\begin{array}{c}w_{3 g} \\
10^{-3} \mathrm{~kg} / \mathrm{s}\end{array}$ & $\begin{array}{l}\mathrm{W}_{31} \\
\mathrm{~kg} / \mathrm{s}\end{array}$ & $\begin{array}{l}d \\
\mathrm{~m}\end{array}$ & $\begin{array}{l}\mathrm{T}_{1} \\
\mathrm{~K}\end{array}$ & $\begin{array}{l}\mathrm{P}_{1} \\
\mathrm{MPa}\end{array}$ & $\begin{array}{c}\Delta \mathrm{P}_{\mathrm{L}-34} \\
\mathrm{MPa}\end{array}$ & $\begin{array}{l}\mathrm{h} \\
\mathrm{m}\end{array}$ & $\begin{array}{c}\mathrm{w}_{3 \mathrm{~g}} \\
10^{-3} \mathrm{~kg} / \mathrm{s}\end{array}$ & $\begin{array}{l}\mathrm{W}_{31} \\
\mathrm{~kg} / \mathrm{s}\end{array}$ \\
\hline \multirow[t]{22}{*}{0.006} & 288 & 0.456 & 0.273 & 0.013 & 4.68 & 0.255 & 0.012 & 280 & 0.437 & 0.050 & 0.015 & 10.4 & 0.482 \\
\hline & & 0.443 & 0.076 & 0.005 & 4.85 & 0.092 & & & 0.476 & 0.033 & 0.017 & 9.57 & 0.442 \\
\hline & & 0.460 & 0.145 & 0.004 & 8.24 & 0.128 & & & 0.417 & 0.035 & 0.019 & 5.81 & 0.488 \\
\hline & & 0.470 & 0.244 & 0.007 & 8.07 & 0.180 & & & 0.475 & 0.030 & & 7.80 & 0.430 \\
\hline & & 0.458 & 0.208 & 0.009 & 6.37 & 0.184 & & 288 & 0.480 & 0.138 & 0.003 & 36.9 & 0.486 \\
\hline & & 0.413 & 0.226 & 0.007 & 6.90 & 0.166 & & & 0.422 & 0.157 & 0.000 & 42.7 & 0.405 \\
\hline & 290 & 0.481 & 0.070 & 0.003 & 6.30 & 0.074 & & 289 & 0.404 & 0.170 & 0.010 & 23.0 & 0.690 \\
\hline & & 0.466 & 0.127 & 0.006 & 6.50 & 0.130 & & & 0.423 & 0.195 & 0.014 & & 0.821 \\
\hline & & 0.444 & 0.250 & 0.004 & 10.2 & 0.136 & & & 0.443 & 0.250 & 0.015 & & 0.917 \\
\hline & & 0.430 & 0.184 & 0.000 & 10.5 & 0.10 & & 296 & 0.455 & 0.205 & 0.004 & 26.0 & 0.635 \\
\hline & & 0.432 & 0.177 & 0.002 & 10.4 & 0.104 & 0.020 & 286 & 0.464 & 0.001 & 0.003 & 5.85 & 0.082 \\
\hline & & 0.485 & 0.155 & 0.012 & 4.15 & 0.211 & & & 0.483 & 0.004 & 0.010 & 9.55 & 0.384 \\
\hline & & 0.463 & 0.182 & 0.011 & 4.35 & & & & 0.441 & 0.003 & 0.013 & 6.87 & 0.408 \\
\hline & & 0.475 & 0.270 & 0.010 & 6.30 & 0.225 & & 287 & 0.445 & 0.008 & 0.006 & 17.6 & 0.345 \\
\hline & 292 & 0.412 & 0.213 & 0.011 & 4.22 & 0.215 & & 292 & 0.426 & 0.018 & 0.011 & 19.1 & 0.692 \\
\hline & & 0.477 & 0.248 & 0.015 & 3.57 & 0.282 & & & 0.474 & 0.023 & 0.013 & 18.8 & 0.808 \\
\hline & & 0.439 & 0.143 & 0.014 & 2.11 & 0.229 & & & 0.442 & 0.032 & 0.016 & 19.1 & 0.969 \\
\hline & & 0.453 & 0.110 & & & 0.191 & & & 0.467 & 0.045 & 0.022 & 18.7 & 1.40 \\
\hline & & 0.460 & 0.077 & 0.009 & 3.23 & 0.136 & & 296 & 0.291 & 0.056 & 0.002 & 35.7 & 0.675 \\
\hline & 293 & 0.482 & 0.200 & 0.012 & 3.19 & 0.246 & & & 0.322 & 0.066 & 0.001 & 48.9 & 0.728 \\
\hline & 294 & 0.372 & 0.091 & 0.006 & 3.51 & 0.111 & & & 0.369 & 0.097 & 0.007 & 54.4 & 1.23 \\
\hline & 290 & 0.412 & 0.070 & & 3.06 & 0.095 & & & 0.374 & 0.102 & 0.013 & 45.3 & 1.46 \\
\hline \multirow[t]{9}{*}{0.012} & 282 & 0.428 & 0.037 & 0.000 & 17.5 & 0.265 & & & 0.367 & 0.109 & 0.016 & 39.1 & 1.57 \\
\hline & & 0.433 & 0.050 & 0.003 & & 0.301 & & & 0.375 & 0.060 & 0.017 & 25.0 & 1.36 \\
\hline & & 0.454 & 0.055 & 0.005 & & 0.265 & & & & 0.414 & & 0.020 & 1.01 \\
\hline & & 0.465 & 0.067 & 0.010 & 13.1 & 0.486 & & & 0.406 & 0.070 & 0.023 & 19.7 & 1.63 \\
\hline & & 0.470 & 0.081 & 0.012 & & 0.590 & & & 0.382 & 0.120 & 0.028 & & 2.34 \\
\hline & 280 & 0.379 & 0.035 & 0.006 & 14.5 & 0.312 & & 288 & 0.382 & 0.008 & 0.004 & & 0.359 \\
\hline & & 0.435 & 0.025 & 0.007 & 13.3 & 0.238 & & & 0.318 & 0.011 & 0.003 & 22.4 & 0.388 \\
\hline & & 0.470 & 0.020 & 0.008 & 12.4 & 0.250 & & & 0.438 & 0.026 & 0.000 & 41.3 & 0.519 \\
\hline & & 0.376 & 0.065 & 0.014 & 11.3 & 0.542 & & & & & & & \\
\hline
\end{tabular}


Table 5.15: Liquid entrainment in horizontal branches

\begin{tabular}{|c|c|c|c|c|c|c|c|c|c|c|c|c|c|}
\hline $\begin{array}{l}d \\
m\end{array}$ & $\begin{array}{r}\mathrm{T}_{1} \\
\mathrm{~K}\end{array}$ & $\begin{array}{l}\mathrm{P}_{1} \\
\mathrm{MPa}\end{array}$ & $\begin{array}{c}\Delta \mathrm{P}_{1-34} \\
\mathrm{MPa}\end{array}$ & $\begin{array}{l}n \\
\text { on }\end{array}$ & $\begin{array}{c}\mathrm{W}_{3 \mathrm{~g}} \\
10^{-3} \mathrm{~kg} / \mathrm{s}\end{array}$ & $\begin{array}{l}\mathrm{N}_{31} \\
\mathrm{~kg} / \mathrm{s}\end{array}$ & $d$ & $\begin{array}{l}T_{1} \\
K\end{array}$ & $\begin{array}{l}\mathrm{P}_{1} \\
\mathrm{MPa}\end{array}$ & $\begin{array}{c}\Delta \mathrm{P}_{1-34} \\
\mathrm{MPa}\end{array}$ & h & $\begin{array}{c}{ }^{W_{3}} 3 \mathrm{~g} \\
10^{-3} \mathrm{~kg} / \mathrm{s}\end{array}$ & $\begin{array}{l}\mathrm{w}_{31} \\
\mathrm{~kg} / \mathrm{s}\end{array}$ \\
\hline \multirow[t]{34}{*}{0.006} & 288 & 0.298 & 0.066 & 0.004 & 7.08 & 0.028 & 0.006 & 288 & 0.423 & 0.125 & 0.005 & 10.8 & 0.054 \\
\hline & & 0.416 & 0.171 & 0.001 & 11.1 & 0.085 & & & 0.419 & 0.096 & 0.007 & & 0.029 \\
\hline & 290 & 0.403 & 0.088 & 0.002 & 9.12 & 0.049 & & & 0.400 & 0.06 & 0.005 & 7.16 & \\
\hline & & 0.369 & 0.084 & 0.001 & 7.99 & 0.051 & & & 0.473 & 0.069 & 0.001 & 6.52 & 0.055 \\
\hline & & 0.383 & 0.102 & 0.003 & 9.36 & 0.052 & & & 0.286 & 0.172 & 0.002 & 7.76 & 0.077 \\
\hline & & 0.360 & 0.095 & 0.006 & 11.3 & 0.020 & & & 0.218 & 0.123 & & 5.70 & 0.062 \\
\hline & & 0.370 & 0.143 & 0.009 & 14.6 & 0.017 & & 295 & 0.476 & 0.132 & 0.001 & 9.16 & 0.092 \\
\hline & & 0.372 & 0.193 & 0.010 & 16.3 & 0.016 & & 297 & 0.346 & 0.199 & & 8.52 & 0.102 \\
\hline & & 0.443 & 0.230 & 0.004 & 15.1 & 0.069 & 0.012 & 279 & 0.336 & 0.121 & 0.028 & 95.2 & 0.003 \\
\hline & & 0.429 & 0.152 & 0.007 & 15.4 & 0.032 & & & 0.384 & 0.188 & 0.030 & 115.0 & 0.002 \\
\hline & & 0.439 & 0.161 & 0.010 & 18.9 & 0.010 & & & 0.435 & 0.041 & 0.010 & 64.6 & 0.109 \\
\hline & 293 & 0.431 & 0.221 & 0.008 & 17.6 & 0.032 & & & 0.416 & 0.243 & 0.019 & 67.8 & 0.151 \\
\hline & & 0.433 & 0.164 & 0.007 & 16.1 & 0.033 & & & 0.438 & 0.254 & & 69.1 & 0.140 \\
\hline & 294 & 0.344 & & 0.001 & 8.56 & 0.087 & & & 0.486 & 0.120 & & 68.2 & 0.121 \\
\hline & 289 & 0.379 & 0.115 & & 7.75 & 0.078 & & & 0.424 & 0.100 & 0.016 & 53.6 & 0.160 \\
\hline & 297 & 0.482 & 0.150 & 0.004 & 11.9 & 0.068 & & & 0.477 & 0.051 & 0.014 & 45.5 & 0.139 \\
\hline & & 0.480 & 0.202 & 0.006 & 14.9 & 0.063 & & & 0.472 & 0.036 & 0.013 & 40.1 & 0.124 \\
\hline & & 0.482 & 0.115 & 0.009 & 15.0 & 0.019 & & 282 & 0.372 & 0.052 & 0.020 & 49.0 & 0.035 \\
\hline & & 0.260 & 0.110 & 0.005 & 7.75 & 0.040 & & & 0.443 & 0.071 & 0.017 & 54.1 & 0.116 \\
\hline & 288 & 0.451 & 0.124 & 0.001 & 10.5 & 0.074 & & & 0.428 & 0.094 & 0.018 & 59.2 & 0.109 \\
\hline & 290 & 0.430 & 0.184 & 0.000 & & 0.105 & & & 0.409 & 0.047 & 0.009 & 36.5 & 0.174 \\
\hline & 287 & 0.190 & 0.094 & 0.001 & 4.33 & 0.056 & & & 0.426 & 0.045 & 0.016 & 46.1 & 0.077 \\
\hline & & 0.180 & 0.090 & 0.004 & 4.40 & 0.054 & & & 0.451 & 0.050 & 0.014 & 45.6 & 0.119 \\
\hline & & 0.298 & 0.180 & 0.008 & 10.6 & 0.042 & & & 0.436 & 0.045 & 0.011 & 38.8 & 0.157 \\
\hline & & 0.374 & 0.220 & 0.009 & 14.6 & 0.032 & & & 0.443 & 0.050 & 0.008 & 30.9 & 0.184 \\
\hline & & 0.448 & 0.114 & & 13.8 & 0.023 & & & 0.409 & 0.069 & 0.016 & 50.8 & 0.096 \\
\hline & & 0.487 & 0.093 & & 13.7 & 0.018 & & 283 & 0.451 & 0.043 & 0.007 & 28.9 & 0.175 \\
\hline & & 0.541 & 0.072 & 0.010 & 12.4 & 0.012 & & & 0.438 & 0.030 & 0.005 & 23.4 & 0.173 \\
\hline & & 0.171 & 0.089 & 0.006 & 5.58 & 0.025 & & & 0.443 & 0.022 & 0.013 & 28.1 & 0.048 \\
\hline & & 0.147 & 0.068 & 0.005 & 4.25 & 0.024 & & & & 0.029 & 0.011 & 28.6 & 0.090 \\
\hline & & 0.203 & 0.117 & 0.008 & 7.60 & 0.021 & & & 0.441 & 0.043 & 0.012 & 37.6 & 0.078 \\
\hline & & 0.230 & 0.084 & & 7.49 & 0.020 & & & 0.451 & 0.039 & 0.018 & 41.5 & 0.024 \\
\hline & & 0.284 & 0.147 & 0.010 & 12.3 & 0.016 & & & 0.429 & 0.046 & 0.009 & 29.9 & 0.166 \\
\hline & 288 & 0.428 & 0.170 & 0.002 & 10.7 & 0.082 & & 282 & 0.428 & 0.019 & 0.004 & 17.1 & 0.155 \\
\hline
\end{tabular}


Table 5.15: (2nd page)

\begin{tabular}{|c|c|c|c|c|c|c|c|c|c|c|c|c|c|}
\hline$d$ & $\begin{array}{l}\mathrm{T}_{1} \\
\mathrm{k}\end{array}$ & $\begin{array}{l}\mathrm{P}_{1} \\
\mathrm{MPa}\end{array}$ & $\begin{array}{c}\Delta \mathrm{P}_{1-34} \\
\mathrm{MPa}\end{array}$ & h & $\begin{array}{c}w_{3 g} \\
10^{-3} \mathrm{~kg} / \mathrm{s}\end{array}$ & $\begin{array}{l}\mathrm{w}_{31} \\
\mathrm{~kg} / \mathrm{s}\end{array}$ & $d$ & $\begin{array}{l}T_{1} \\
k\end{array}$ & $\begin{array}{l}\mathrm{P}_{1} \\
\mathrm{MPa}\end{array}$ & $\underset{1-34}{\Delta \mathrm{Pa}^{\Delta \mathrm{P}}}$ & $\begin{array}{l}\mathrm{h} \\
\mathrm{m}\end{array}$ & $\begin{array}{c}\mathrm{w}_{3 \mathrm{~g}} \\
10^{-3} \mathrm{~kg} / \mathrm{s}\end{array}$ & $\begin{array}{l}\mathrm{w}_{31} \\
\mathrm{~kg} / \mathrm{s}\end{array}$ \\
\hline \multirow[t]{23}{*}{0.012} & 282 & 0.434 & 0.019 & 0.002 & 15.0 & 0.167 & 0.020 & 292 & 0.323 & 0.050 & 0.023 & 94.0 & 0.164 \\
\hline & & 0.345 & 0.030 & 0.001 & 15.4 & 0.230 & & & 0.384 & 0.030 & 0.001 & 40.5 & 0.459 \\
\hline & & 0.428 & 0.037 & 0.000 & 17.5 & 0.265 & & & 0.462 & 0.017 & & 39.6 & 0.44 \\
\hline & 296 & 0.470 & 0.187 & 0.001 & 36.2 & 0.477 & & 295 & 0.435 & 0.060 & 0.025 & 115.4 & 0.135 \\
\hline & 295 & 0.492 & 0.079 & & 28.6 & 0.305 & & & 0.477 & 0.065 & 0.011 & 81.22 & 0.498 \\
\hline & & 0.441 & 0.094 & & 29.5 & 0.335 & & & 0.463 & 0.040 & 0.021 & 88.0 & 0.195 \\
\hline & & 0.410 & 0.153 & & 35.15 & 0.432 & & & 0.384 & 0.070 & 0.017 & 89.1 & 0.358 \\
\hline & & 0.379 & 0.185 & & 35.4 & 0.450 & & & 0.482 & 0.013 & 0.006 & 41.03 & 0.283 \\
\hline & & 0.323 & 0.113 & & 26.4 & 0.359 & & & 0.419 & 0.028 & 0.010 & 62.5 & 0.324 \\
\hline & & 0.242 & 0.103 & & 19.3 & 0.335 & & & 0.342 & 0.045 & 0.012 & 73.8 & 0.368 \\
\hline & 289 & 0.218 & 0.018 & & 9.58 & 0.149 & & & 0.325 & & 0.018 & 79.9 & 0.239 \\
\hline & & 0.254 & 0.021 & 0.002 & 11.7 & 0.144 & & & 0.311 & 0.040 & 0.024 & 86.2 & 0.089 \\
\hline & & 0.141 & 0.014 & 0.004 & 7.07 & 0.087 & & & 0.387 & 0.010 & 0.004 & 28.1 & 0.307 \\
\hline & & 0.422 & 0.006 & & 9.60 & 0.063 & & & 0.412 & 0.016 & 0.001 & 34.7 & 0.401 \\
\hline & & 0.546 & 0.007 & & 12.8 & 0.073 & & 288 & 0.308 & 0.010 & & 23.5 & 0.255 \\
\hline & & 0.402 & 0.004 & 0.002 & 7.29 & 0.067 & & & 0.412 & 0.005 & & 22.9 & 0.219 \\
\hline & & 0.487 & 0.005 & 0.006 & 11.1 & 0.040 & & & 0.451 & 0.004 & 0.003 & 22.5 & 0.166 \\
\hline & & 0.157 & 0.037 & 0.012 & 17.5 & 0.067 & & 289 & 0.305 & 0.006 & 0.006 & 37.3 & 0.305 \\
\hline & & 0.159 & 0.054 & 0.014 & 19.6 & 0.043 & & & 0.327 & 0.029 & & 48.9 & 0.397 \\
\hline & & 0.495 & 0.006 & 0.012 & 16.6 & 0.012 & & & 0.402 & 0.022 & 0.005 & 48.6 & 0.362 \\
\hline & & 0.172 & 0.008 & 0.014 & 17.9 & 0.006 & & & 0.462 & 0.028 & 0.001 & 48.0 & 0.505 \\
\hline & & 0.296 & 0.014 & & 18.7 & 0.015 & & & 0.334 & 0.050 & 0.016 & 76.7 & 0.226 \\
\hline & 288 & 0.422 & 0.157 & 0.000 & 42.7 & 0.405 & & & 0.463 & 0.026 & 0.015 & 74.8 & 0.188 \\
\hline \multirow[t]{5}{*}{0.020} & 287 & 0.441 & 0.007 & 0.010 & 36.5 & 0.145 & & & 0.335 & 0.045 & 0.011 & 74.0 & 0.381 \\
\hline & & 0.475 & 0.007 & 0.007 & 35.4 & 0.197 & & & 0.304 & 0.065 & 0.014 & 81.1 & 0.393 \\
\hline & & 0.480 & & 0.001 & 26.6 & 0.292 & & & 0.353 & 0.070 & 0.009 & 83.5 & 0.589 \\
\hline & & 0.462 & 0.008 & 0.004 & 31.7 & 0.267 & & & 0.409 & 0.090 & 0.001 & 83.3 & 0.877 \\
\hline & & 0.492 & & 0.015 & 45.4 & 0.102 & & 288 & 0.438 & 0.026 & 0.000 & 41.3 & 0.519 \\
\hline
\end{tabular}

